\title{
The Effect of Opiates on the Developing Cerebral Cortex
}

\author{
By
}

\section{Timothy John Sargeant}

A thesis submitted to the Victoria University of Wellington in fulfilment of the requirements for the degree of Doctor of Philosophy

in Cell and Molecular Bioscience

Victoria University of Wellington

2008 


\section{Abstract}

Opiate drugs, such as codeine, morphine and heroin are powerful analgesics and drugs of abuse. The unborn child is invariably exposed to opiate drugs as a consequence of maternal use. Studies that have investigated the impact of opiate drugs demonstrated opioid system expression in proliferating regions of the developing brain, as well as on proliferative astroglia taken from the developing central nervous system. The effects of opiates on astroglial proliferation (largely mediated by the mu opioid receptor) are predominantly inhibitory, but are extremely context dependent. This context dependency exists because of the complexity resident within the opioid signalling system. However, since this previous research was conducted, there has been impressive progress made in the field of developmental neurobiology with the demonstration that cells of astrocytic lineage are responsible for the generation of the central nervous system.

It was therefore the aim of the current research project to investigate the developmental impact of opiate exposure in the context of the foetal mouse cerebral cortex. This aim was divided into 3 separate aims that comprised of; determining the cellular localisation of the mu opioid receptor, the effects of opiate exposure on cortical progenitor cells, and to determine the effect of opiate exposure on the development of the cerebral cortex itself. The mu opioid receptor was expressed on proliferative radial glia of both the embryonic day 15.5 (neurogenic) and embryonic day 18.5 (gliogenic) ventricular zone of the dorsal forebrain. Interestingly and significantly, the mu opioid receptor-positive glia observed in the embryonic day 18.5 mouse forebrain were also observed at a comparable 
developmental stage in the foetal human forebrain. Morphine exposure slowed down $\mathrm{G}_{2}$ phase of the cell cycle at embryonic day 15.5 in the neurogenic murine cortical ventricular zone. This opiate-induced slowing in cell cycle progression was shown not to impact on proliferation in the ventricular zone, although future research should address whether this perturbation altered differentiation or developmental maturation of the radial glia. Morphine exposure throughout corticogenesis decreased levels of doublecortin expression (a migratory neuronal marker) at the end of gestation. Postnatally, mice exposed to morphine during corticogenesis also showed decreased numbers of neurons in layer $\mathrm{V}$ of the cerebral cortex.

Collectively, this thesis presents the first evidence that shows morphine affects cortical progenitor cells in vivo. This research supports the possibility that the opioid system plays an endogenous role in corticogenesis. The clinical significance is morphine has the potential to perturb normal development of the cerebral cortex. 


\section{Acknowledgments}

Dr Darren J Day has provided endless enthusiasm and ideas which have grown this project. Your pizzas and help at 3 am were much appreciated.

Thank you Prof. John H Miller for your invaluable input, reading my writing and helping with our many dissections.

Thank you to my lovely partner, Elizabeth, for dragging me out of bed every day at 6 am and your support at home. You shaved years off of my project.

Thank you Dr Bronwyn M Kivell for establishing the new confocal facility at Victoria. Also thank you for your support in my contribution to the wider school community.

Thank you Dr Al Rowland and Dr Sara Filoche for providing access to confocal facilities at the beginning of my project. Thank you Peter Smith, Cameron Jack and Alan Hoverd for technical advice on microscopy and histology.

Thank you to the academic staff of SBS, especially Dr Anne La Flamme and Dr Bill Jordan, as well as the administrative staff of the School of Biological Science, Mary Murray and Patricia Stein in particular.

Thanks to people who have passed through KK801 - Anasuya Vishvanath, Dr Catherine Seamer, Babs Lake, Ange Fleming, Dr Eli Mrkusich, Hongjun Shi, Darren Foo, Tom Fanning and Ryan Steel - as well as KK601

- Dr Kevin Crume (thanks for coaching me at the gym!), James Matthews, Amy Lewis TPC and Anja Wilmes.

Thank you to my fantastic family and God.

This project was supported by funding from the Wellington Medical Research Foundation, Inc., and a Bright Future PhD scholarship from the Tertiary Education Commission. 


\section{Table of Contents}

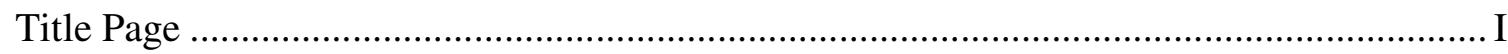

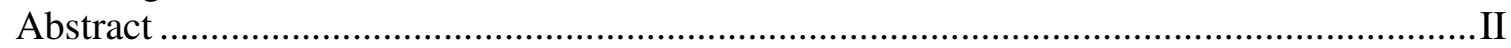

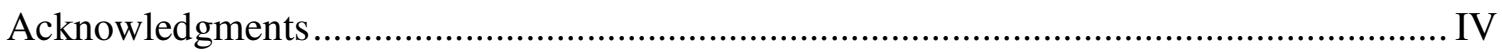

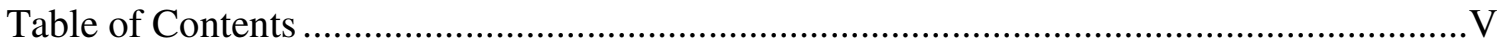

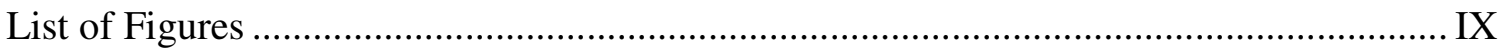

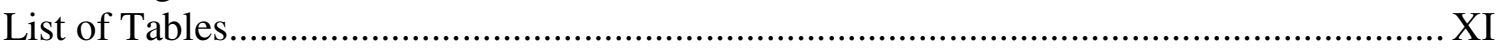

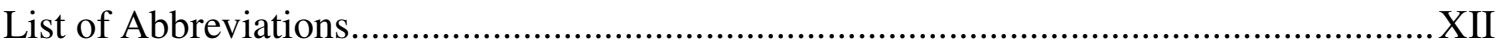

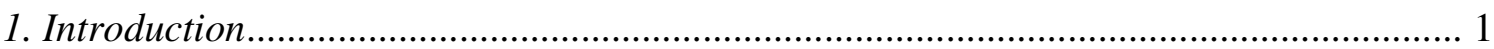

1.1 The Opioid Signalling System: Historical Aspects ..................................................... 1

1.2 Molecular Biology and Biochemistry of the Opioid Receptors ................................. 3

1.2.1 Molecular Biology of the Opioid receptors ...................................................... 3

1.2.2 Phamacological Variants of the Opioid Receptors.............................................. 8

1.2.3 Homo- and Heterodimerisation of the Opioid Receptors ................................... 11

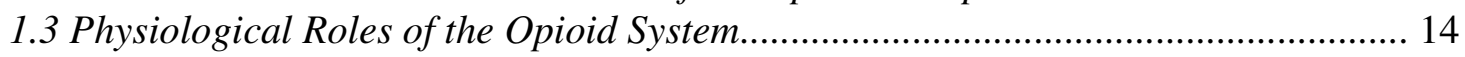

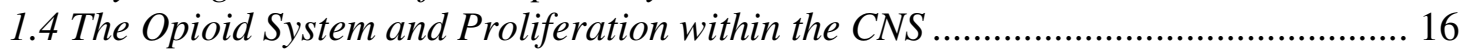

1.4.1 Opioid Signalling System Expression in the Foetal Brain.................................. 16

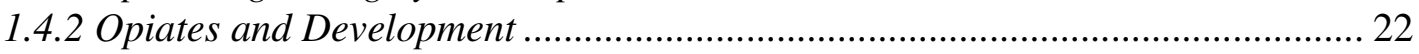

1.4.3 Opiates and Cellular Proliferation in the CNS................................................. 25

1.4.4 Met-Enkephalin (Opioid Growth Factor) and OGFr ...................................... 32

1.4.5 Other Opioid Effects on Neuronal Cells in the Developing Brain...................... 33

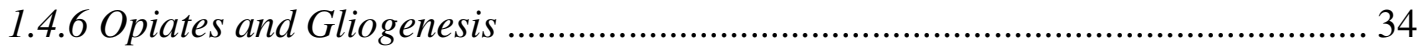

1.4.7 Opioid Receptor Signalling Pathways Associated With Proliferation ................ 37

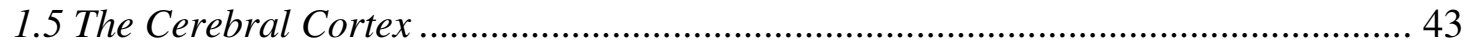

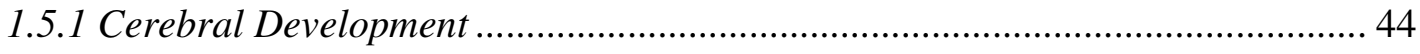

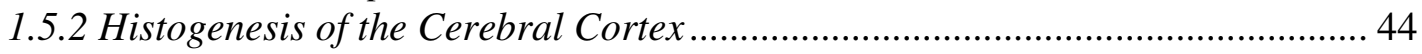

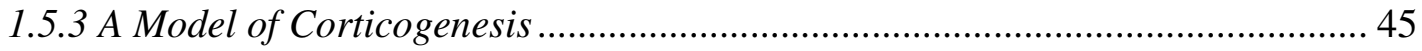

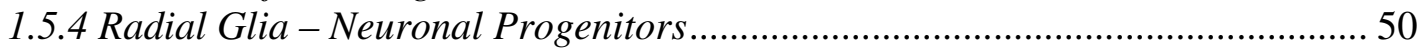

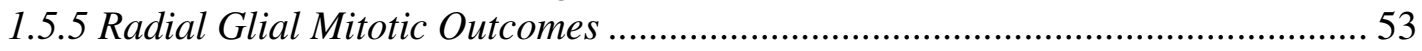

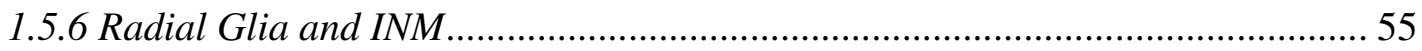

1.5.7 Role of the SVZ in Foetal Corticogenesis ......................................................... 58

1.5.8 Postnatal Neurogenesis in the Mammalian Forebrain.................................... 59

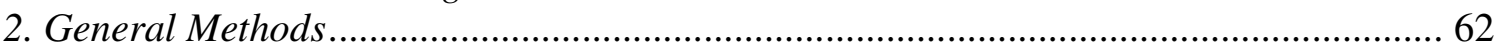

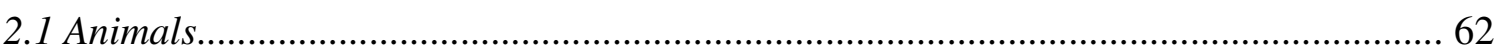

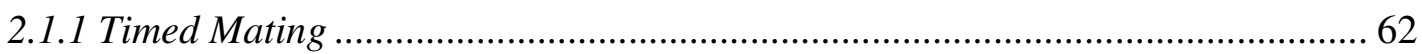

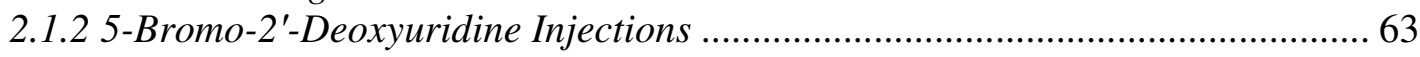

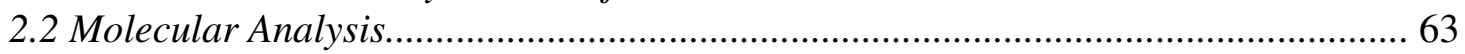

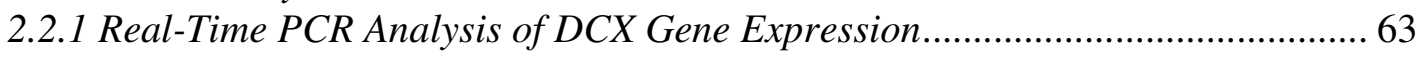

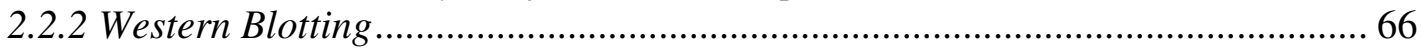

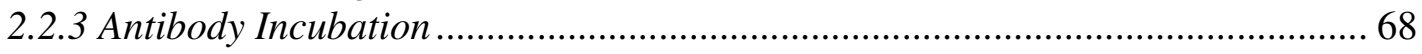

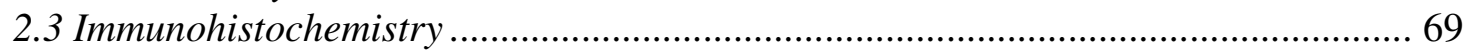

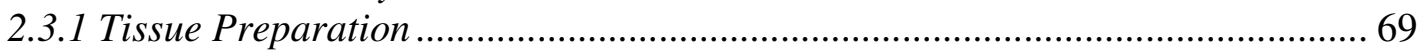

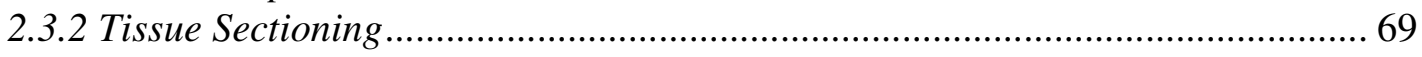

2.3.3 Fluorescence Immunohistochemistry for Fixed-Frozen Brain Sections ............. 70 
2.3.4 BrdU Staining for Fixed-Frozen Sections.

2.3.5 Fluorescence Immunohistochemistry for Paraffin-Embedded Sections .............. 72

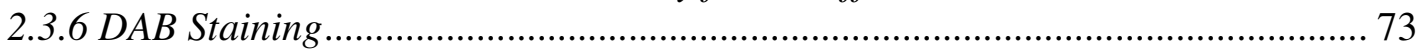

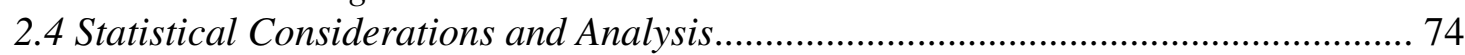

2.4.1 The Use of Multiparous Species in Developmental Studies.............................. 74

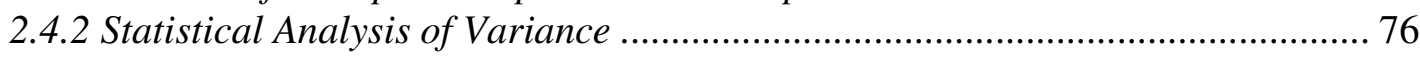

3. MOR is Expressed on Radial Glia but not on Migrating Neurons in the Late Foetal

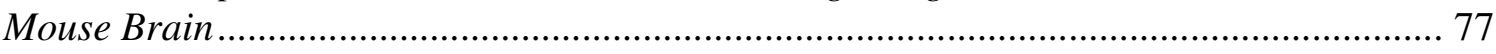

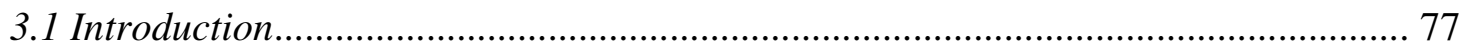

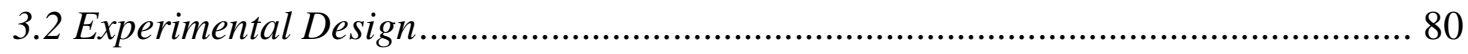

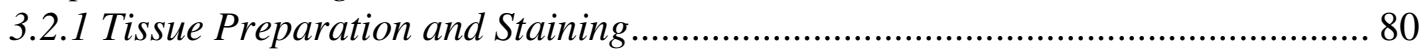

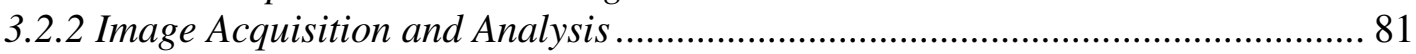

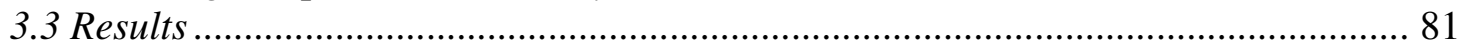

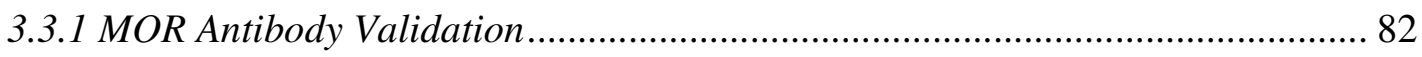

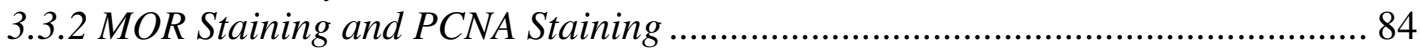

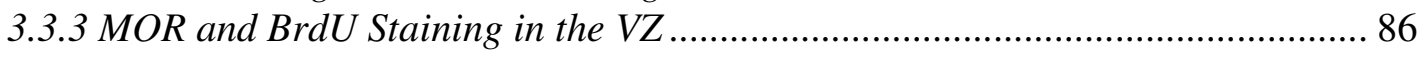

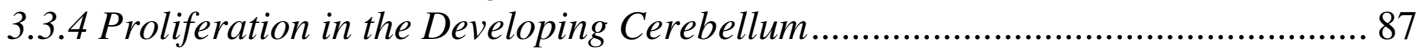

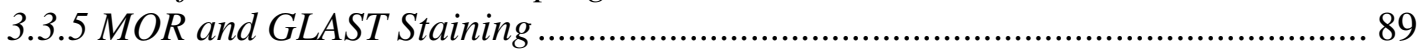

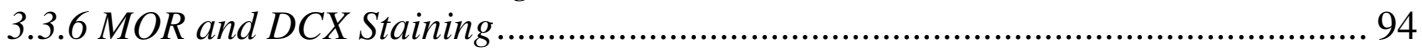

3.3.7 Gradient of Proliferation and MOR Expression within the LV Cortical

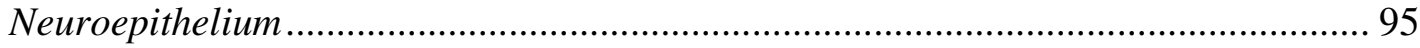

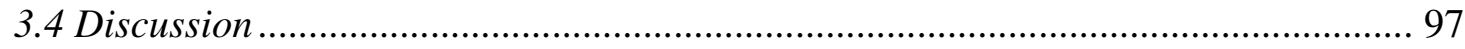

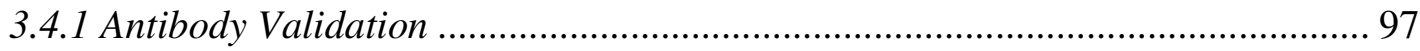

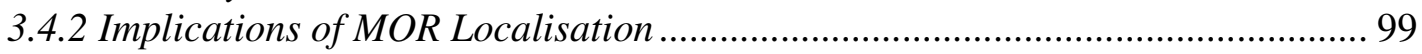

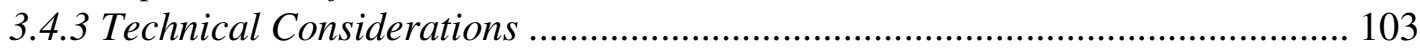

4. MOR is Expressed on Glia of the Foetal Human Forebrain ......................................... 105

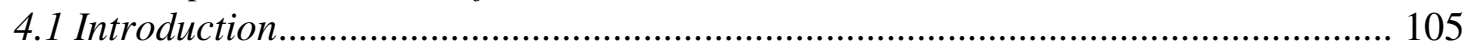

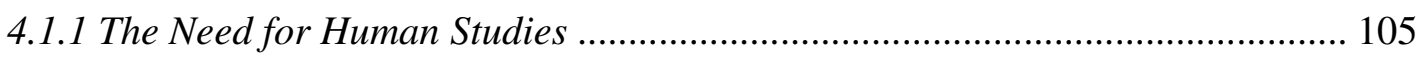

4.1.2 Anatomical and Cellular Contrasts between Human and Rodent Corticogenesis

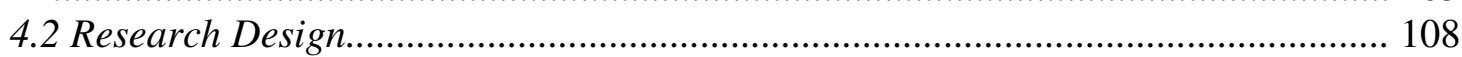

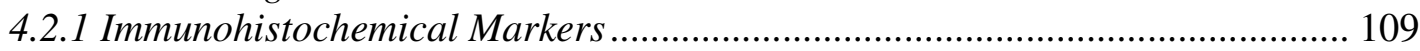

4.2.2 Tissue Processing and Image Acquisition.................................................... 109

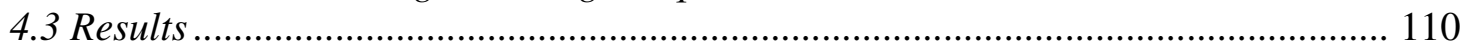

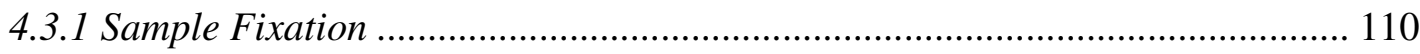

4.3.2 Human Foetal Brain Sample and Reconstruction ......................................... 111

4.3.3 Opioid Receptor Antibody Staining in the Human Brain ................................. 112

4.3.4 MOR Co-labelling in the Foetal Human Dorso-Lateral SVZ........................... 114

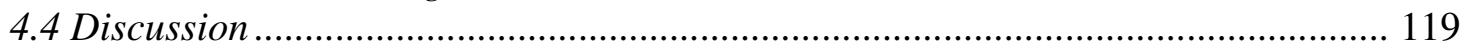

5. Acute Morphine Exposure Lengthens Cell Cycle within the Dorsal Forebrain of the

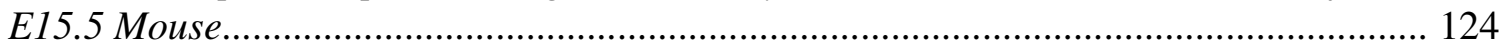

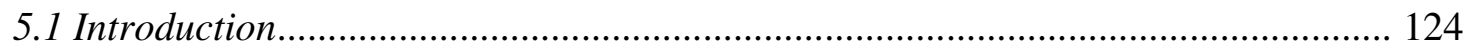

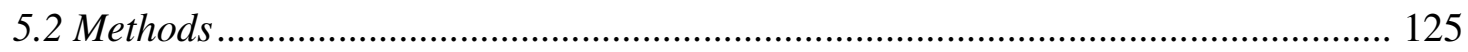

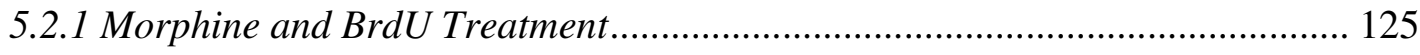

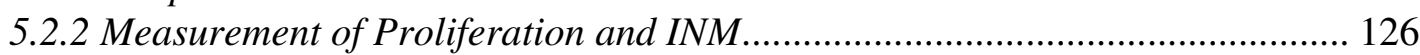

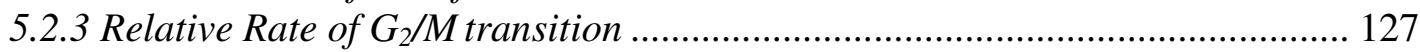


5.2.4 Morphine Treatment Effect on the Duration of the Cell Cycle and Exit from the

Proliferative Pool 127

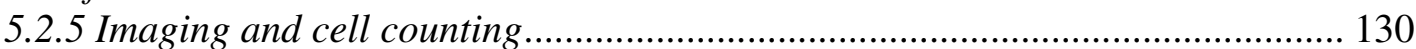

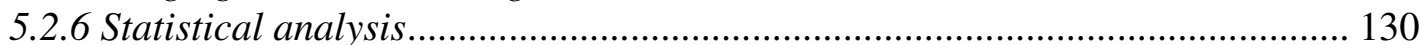

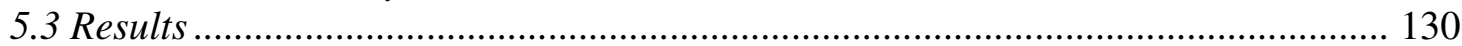

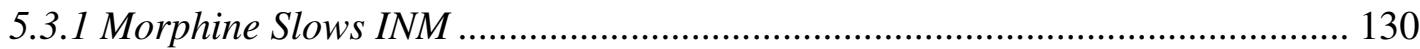

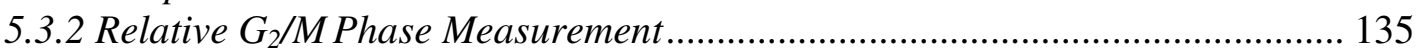

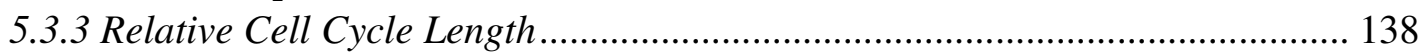

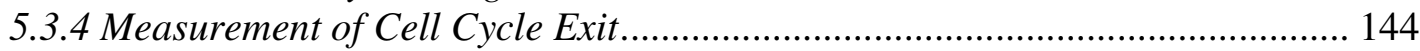

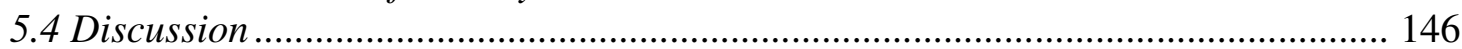

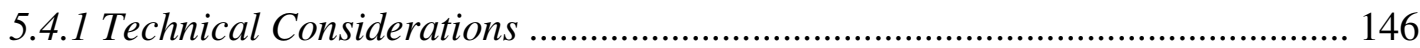

6. Chronic Morphine Exposure and Histogenesis of the Cerebral Cortex........................ 158

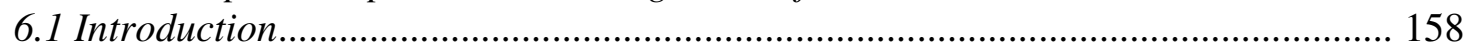

6.1.1 Chronic Opiate Treatment and Effects on Cellular Proliferation .................... 158

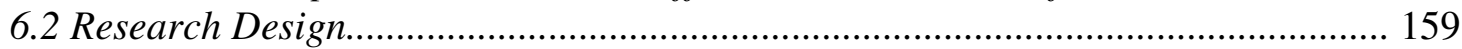

6.2.1 Chronic Morphine Administration ................................................................... 159

6.2.2 Morphine Effect on Weight Gain in the Pregnant Dam and Mouse Pup ......... 160

6.2.3 Effects of Chronic Morphine Treatment on Proliferation and Neuronal Migration in the Foetal Cerebral Cortex ................................................................. 161

6.2.4 Real-time PCR Analysis of the Chronically Morphine Exposed E18.5 Cerebral

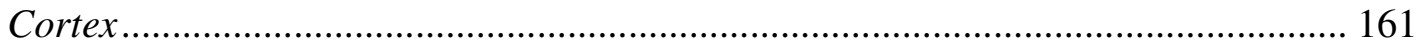

6.2.5 Western Blot Analysis of DCX in the E18.5 Cerebral Cortex ......................... 162

6.2.6 Counting DCX Positive Neurons in the E18.5 Cerebral Cortex....................... 162

6.2.7 Western Blot Analysis of P5 Cerebral Cortex................................................ 164

6.2.8 Stereological Analysis of Nissl-Stained Neurons in the P5 Neocortex............. 164

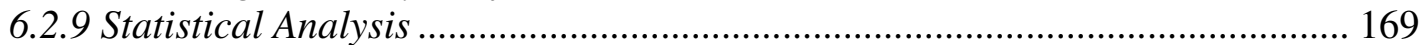

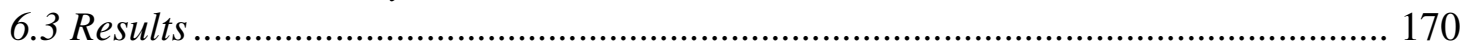

6.3.1 Chronic Morphine Exposure and Weight Gain in Pregnant Dams .................. 170

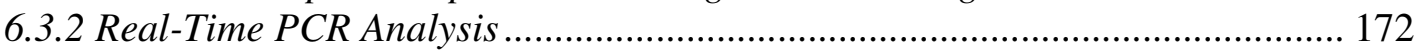

6.3.3 Effect of Chronic Morphine Exposure on Cellular Proliferation and Neuronal

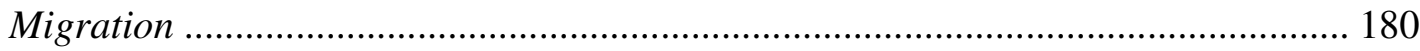

6.3.4 DCX-Positive Cell Counting in the E18.5 Cerebral Cortex ............................. 181

6.3.5 Analysis of the P5 Cerebral Cortex - Western Blotting .................................. 183

6.3.6 Stereological Cell Counting in the P5 Neocortex ........................................... 184

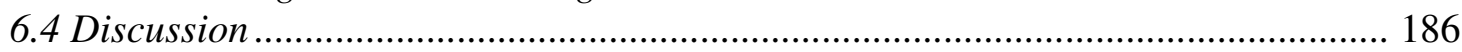

6.4.1 Chronic Morphine Administration does not Decrease Weight in the Perinatal

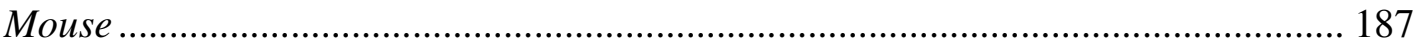

6.4.2 Morphine Induced Down-Regulation of DCX Expression................................ 188

6.4.3 Chronic Morphine and Loss of Cortical Neurons in the Postnatal Pup........... 190

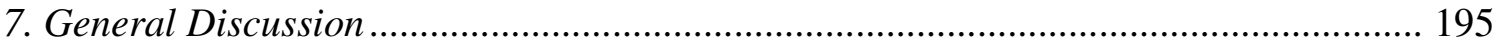

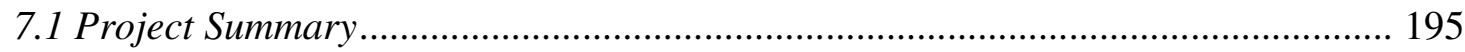

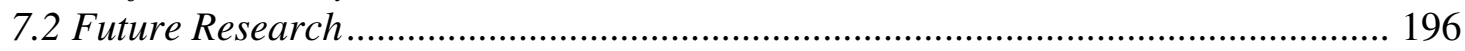

7.2.1 Remodel the In Vivo Experiments in an In Vitro Setting ................................. 196

7.2.2 Use Opioid Antagonists to Identify Receptor Subtypes and Endogenous Activities 
7.2.4 Investigate Different Treatment Regimes - Continuous Administration by Osmotic Pumps

7.2.5 Use Other Clinically Relevant Opiates ............................................................. 201

7.3 Weaknesses in the Field of Opioids and Developmental Biology........................... 202

7.4 An Integrated Model of Opioidergic Signalling and Cortical Development ............ 206

7.5 What is the Significance of Opioid Signalling in Development of the CNS?........... 208

7.5.1 A Biological Role for the Endogenous Opioid System in Corticogenesis?....... 208

7.5.2 A Highly Utilised Drug with Lasting Consequences?..................................... 209

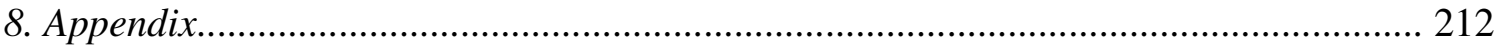

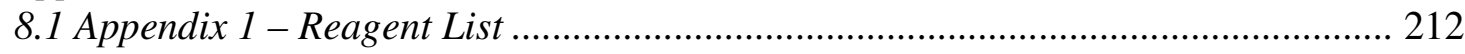

8.2 Appendix 2 - Nissl Staining Brain Sections....................................................... 216

8.3 Appendix 3 - Optimisation of BrdU Staining on Frozen Tissue Sections................. 218

8.4 Appendix 4 - Sectioning and Nissl Staining of 12 GW Human Brainstem............... 220

8.5 Appendix 5 - Pilot Experiment of the Effects of Morphine on INM ....................... 222

8.6 Appendix 6 - Calculation of Likely Concentration of Peak Plasma Morphine....... 223

8.7 Appendix 7 - The Effect of Morphine on Growth of Different Cell Lines as Measured

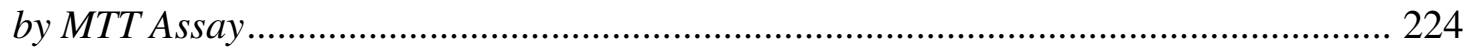

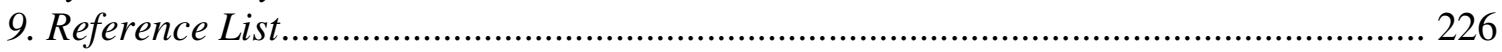

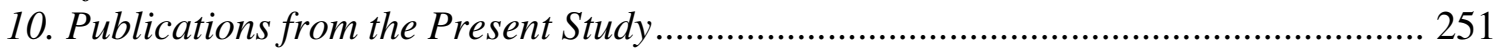

Sargeant et al. (2007)

Sargeant et al. (2008) 


\section{List of Figures}

Fig. 1.1. A stereo-image of MOR, as seen from an extracellular view point ....................... 4

Fig. 1.2. Neuronal migration and nomenclature in the developing cerebral cortex ............. 51

Fig. 1.3. INM and neurogenic divisions within the dorsal telencephalon............................5 56

Fig. 1.4. A simplified diagram of neuronal lineage ........................................................... 59

Fig. 3.1. MOR mRNA expression in the VZ of the E16 rat telencephalon ........................ 78

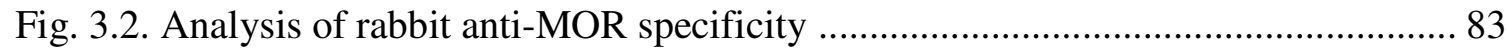

Fig. 3.3. MOR and PCNA IR in the E18.5 neuroepithelia of the LV, $3 \mathrm{~V}$, and Aq ............ 85

Fig. 3.4. MOR and BrdU IR in the E15.5 and E18.5 VZ.............................................. 87

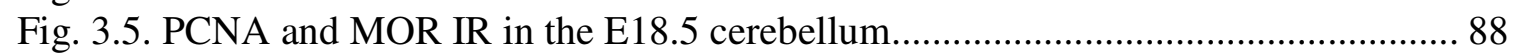

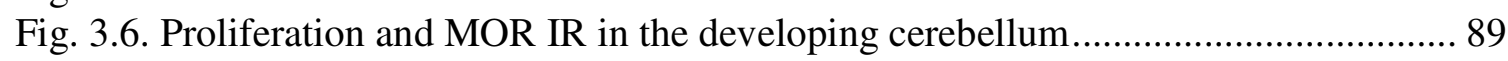

Fig. 3.7. MOR and GLAST IR in the E15.5 and E18.5 LV neuroepithelium .................... 91

Fig. 3.8. DOR immunoreactivity in the E15.5 VZ ...................................................... 92

Fig. 3.9. MOR and GLAST IR in the neuroepithelia of the $3 \mathrm{~V}$ and $\mathrm{Aq}$ in the E18.5 mouse

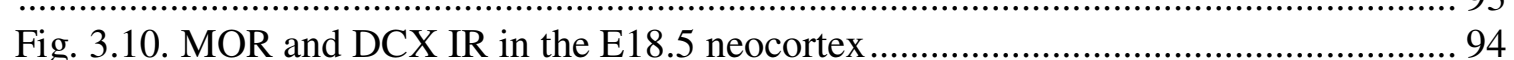

Fig. 3.11. Gradients of proliferation and MOR expression in the dorsal VZ of the LV in

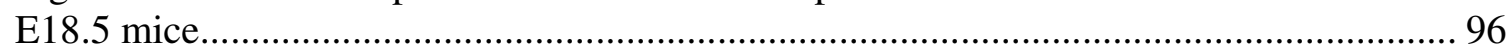

Fig. 4.1. Human foetal brain samples on arrival from Australia...................................... 111

Fig. 4.2. Photography and identification of a main structures ......................................... 112

Fig. 4.3. Opioid receptor staining in the $16 \mathrm{GW}$ human striatum................................. 113

Fig.4.4. MOR staining at the LV surface in the human and in the mouse ......................... 114

Fig. 4.5. Vimentin staining of the radial glial scaffold at $19 \mathrm{GW}$ in the developing human

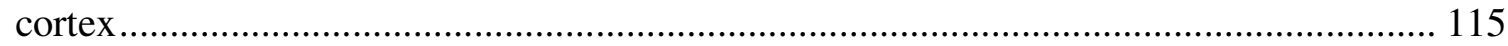

Fig. 4.6. Cellular co-expression of MOR and the markers vimentin, nestin and DCX .... 116

Fig. 4.7. Orthogonal views of cells co-expressing MOR IR and vimentin or nestin ......... 117

Fig. 4.8. A graph showing proportions of MOR expressing cells that co-express the markers

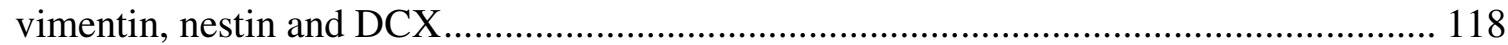

Fig. 5.1. Diagrams of BrdU pulse-chase experiments .................................................... 129

Fig. 5.2. Acute morphine treatment slows INM in the VZ of the LV in the E15.5 mouse 132

Fig. 5.3. Histogram plots showing INM of BrdU-labelled nuclei within the VZ ............. 133

Fig. 5.4. Morphine does not alter total BrdU labelling in E15.5 mouse foetuses but does

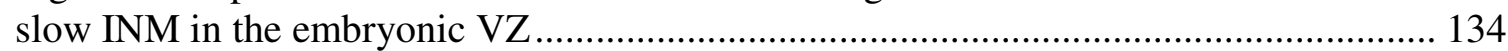

Fig. 5.5. Morphine treatment slows G2/M progression in the E15.5 pup......................... 137

Fig. 5.6. Morphine treatment decreases BrdU/phospho-histone $\mathrm{H} 3$ co-labelling $16 \mathrm{~h}$ after

BrdU administration in basal and apical progenitors .................................................. 140

Fig. 5.7. Morphine treatment does not change the number of mitoses or the proportion of

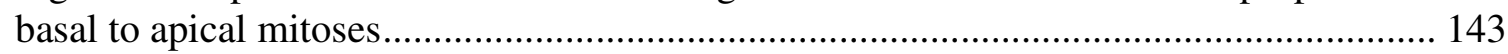

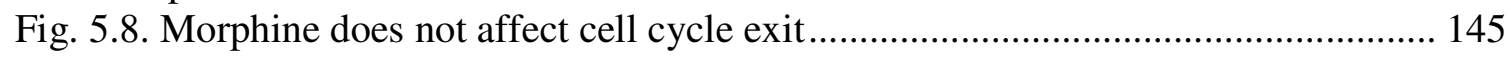

Fig. 5.9. BrdU labelling of G2 phase and mitotic nuclei ............................................ 151

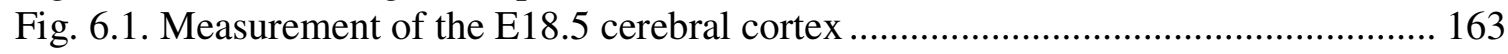

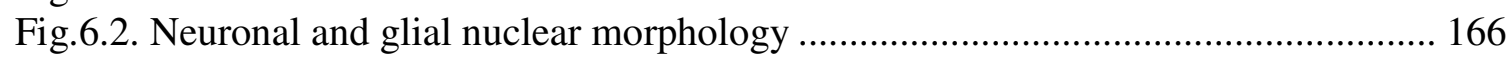

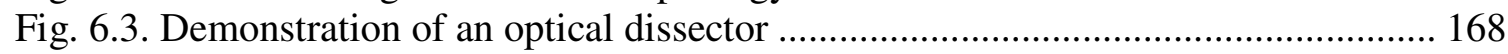

Fig. 6.4. The effects of chronic morphine exposure, in utero, on weight gain in the pregnant

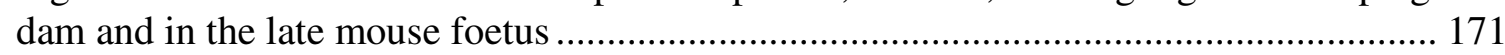


Fig. 6.5. The effects of chronic in utero morphine exposure on the weight of cross-fostered

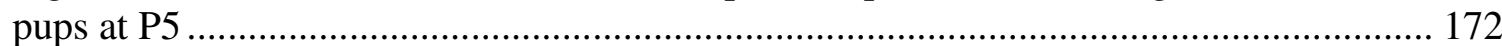

Fig. 6.6. Quantitative comparison of Cyclophilin A with other housekeeping genes ....... 173

Fig. 6.7. Graphs used to determine primer efficiency values.......................................... 174

Fig. 6.8. Chronic morphine treatment during corticogenesis decreases DCX expression in

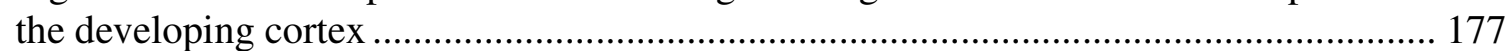

Fig. 6.9. Fas mRNA expression does not change between saline and morphine-treated

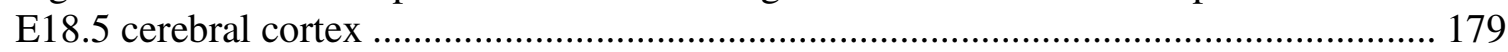

Fig. 6.10. Morphine does not have dramatic affects on total proliferation in the E18.5 dorsal forebrain

Fig. 6.11. Chronic morphine treatment does not induce major abnormalities in neuronal migration

Fig 6.12. DCX-positive cell counting and thickness of the E18.5 cerebral cortex

Fig. 6.13. Chronic morphine exposure from E11.5 to E18.5 does not change expression of

neuronal and glial markers in the cerebral cortex ......................................................... 184

Fig. 6.14. Stereological analysis of the cerebral cortex of the P5 mouse ......................... 186

Fig. 8.1. Optimisation of BrdU staining of fixed-frozen tissue .................................. 218

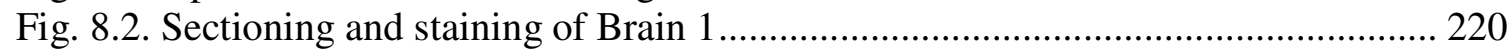

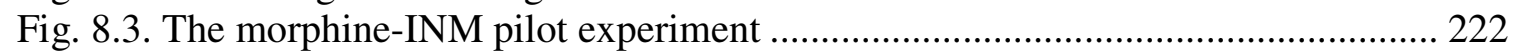

Fig. 8.4. Morphine and growth of cell cultures............................................................... 224 


\section{List of Tables}

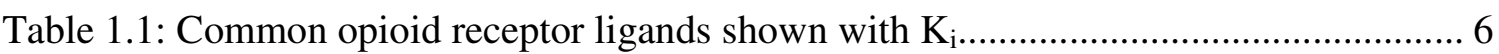

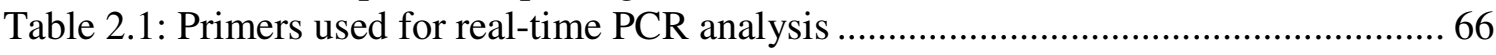

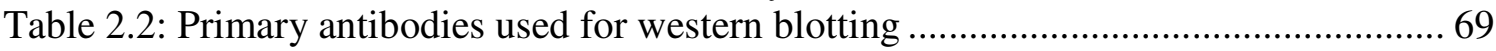

Table 2.3: Primary antibodies used for immunohistochemistry ....................................... 74

Table 2.4: Secondary antibodies used for immunohistochemistry .................................... 74

Table 3.1: Studies that have reported glycosylated forms of the MOR .............................. 98

Table 4.1: Fixation parameters for the brain samples obtained from As. Prof. T.C-L. ..... 110

Table 6.1. Primer sets shown with associated primer efficiency values ............................ 175

Table 7.1. Commercially available selective opioid receptor antagonists (Tocris Bioscience, 2007) 


\section{List of Abbreviations}

\begin{tabular}{|c|c|}
\hline${ }^{3} \mathrm{H}$ & Tritiated \\
\hline $3 \mathrm{~V}$ & Third Ventricle \\
\hline $\mathrm{ABC}$ & Avidin-Biotin Complex \\
\hline ADAM & A Disintegrin And Metalloproteinase \\
\hline AHP & Adult Hippocampal Progenitor \\
\hline ANCOVA & Analysis of Co-Variance \\
\hline ANOVA & Analysis of Variance \\
\hline $\mathrm{Aq}$ & Aqueduct \\
\hline BCA & Bicinchoninic Acid \\
\hline Beta-FNA & Beta-Funaltrexamine \\
\hline BLBP & Brain-Lipid Binding Protein \\
\hline BrdU & 5-bromo-2'-deoxyuridine \\
\hline BSA & Bovine Serum Albumin \\
\hline CDK & Cyclin Dependent Kinase \\
\hline cDNA & Copy DNA \\
\hline cne & Cortical Neuroepithelium \\
\hline CNS & Central Nervous System \\
\hline $\mathrm{CP}$ & Cortical Plate \\
\hline CREB & cAMP Response Element Binding Protein \\
\hline $\mathrm{Ct}$ & Threshold Cycle \\
\hline CTOP & D-Phe-Cys-Tyr-D-Trp-Orn-Thr-Pen-Thr-NH2 \\
\hline DAB & Diaminobenzidine \\
\hline DADLE & {$\left[\mathrm{D}-\mathrm{Ala}^{2}, \mathrm{D}-\mathrm{Leu}^{5}\right]$ enkephalin } \\
\hline DAMGO & [D-Ala2,NMePhe4,Gly-ol]enkephalin \\
\hline DAPI & 4',6-diamidino-2-phenylindole \\
\hline DCX & Doublecortin \\
\hline DiI & 1,1'-dioctadecyl-3,3,3'3'-tetramethylindocarbocyanine perchlorate \\
\hline DNA & Deoxyribonucleic Acid \\
\hline DOR & Delta Opioid Receptor \\
\hline DPDPE & {$\left[\mathrm{D}-\right.$ pen $^{2}, \mathrm{D}-$ pen $\left.^{5}\right]$ enkephalin } \\
\hline DSLET & [D-ser $\left.{ }^{8}-l e u\right]$ enkephalin[Thr] \\
\hline $\mathrm{E}$ & Embryonic Day (as in E15) \\
\hline $\mathrm{EC}_{50}$ & Effective Concentration (50\%) \\
\hline EGF & Epidermal Growth Factor \\
\hline EGFr & Epidermal Growth Factor Receptor \\
\hline EGL & External Granule Layer \\
\hline ERK & Extracellular-Signal Regulated Kinase \\
\hline $\mathrm{G}_{0}$ & Quiescent phase of the cell cycle \\
\hline $\mathrm{G}_{1}$ & Gap 1 phase of the cell cycle \\
\hline $\mathrm{G}_{2}$ & Gap 2 phase of the cell cycle \\
\hline $\mathrm{G}_{2} / \mathrm{M}$ & Gap 2/Mitosis \\
\hline GABA & Gamma Amino Butyric Acid \\
\hline GAD & Glutamic Acid Decarboxylase \\
\hline
\end{tabular}




\begin{tabular}{|c|c|}
\hline GAPDH & Glyceraldehyde Phosphate Dehydrogenase \\
\hline GFAP & Glial Fibrillary Acidic Protein \\
\hline GLAST & Astrocyte Specific Glutamate Transporter \\
\hline GLM & General Linear Model \\
\hline GPCR & G-Protein Coupled Receptor \\
\hline GW & Gestational Week \\
\hline hGFAP & Human Glial Fibrillary Acidic Protein \\
\hline HRP & Horse Radish Peroxidase \\
\hline IGL & Internal Granule Layer \\
\hline $\mathrm{IHC}$ & Immunohistochemistry \\
\hline INM & Interkinetic Nuclear Migration \\
\hline IR & Immunoreactivity \\
\hline $\mathrm{IZ}$ & Intermediate Zone \\
\hline $\mathrm{K}_{\mathrm{d}}$ & Dissociation Constant \\
\hline $\mathrm{K}_{\mathrm{i}}$ & Inhibitory Constant \\
\hline $\mathrm{KO}$ & Knock Out \\
\hline KOR & Kappa Opioid Receptor \\
\hline LDS-PAGE & Lithium Dodecyl Sulfate-Polyacrylamide Gel Electrophoresis \\
\hline LV & Lateral Ventricle \\
\hline MAP & Mitogen Activated Protein (Kinase) \\
\hline MOR & Mu Opioid Receptor \\
\hline MTT & $\begin{array}{l}\text { 3-(4,5-Dimethylthiazol-2-yl)-2,5-diphenyltetrazolium bromide } \\
\text { (tetrazole) }\end{array}$ \\
\hline MZ & Marginal Zone \\
\hline $\mathrm{NE}$ & Neuroepithelium \\
\hline NeuN & Neuronal Nuclear Antigen \\
\hline OGF & Opioid Growth Factor \\
\hline OGFr & Opioid Growth Factor Receptor \\
\hline ORL1 & Opioid Receptor Like1 \\
\hline $\mathrm{P}$ & Postnatal Day (as in P5) \\
\hline $\mathrm{P}$ & Proliferative \\
\hline PBS & Phosphate Buffered Saline \\
\hline PCNA & Proliferating Cell Nuclear Antigen \\
\hline PCR & Polymerase Chain Reaction \\
\hline PFA & Paraformaldehyde \\
\hline PI3K & Phosphoinositide 3 Kinase \\
\hline $\mathrm{Pk} / \mathrm{Mo}$ & Purkinje/Molecular Cell Layer \\
\hline PKC & Protein Kinase C \\
\hline PLC & Phospholipase C \\
\hline Q & Quiescent \\
\hline $\mathrm{RC} 2$ & Radial Cell 2 \\
\hline RFU & Relative Fluorescence Units \\
\hline RIPA & Radio-Immuno Precipitation Assay \\
\hline S phase & DNA Synthesis Phase \\
\hline s.c. & Subcutaneous \\
\hline SDS & Sodium Dodecyl Sulfate \\
\hline
\end{tabular}


SEM

Str

SVZ

TAE

VZ

Standard Error of the Mean

Striatum

Subventricular Zone

Tris Acetate EDTA

Ventricular Zone 


\section{Introduction}

\subsection{The Opioid Signalling System: Historical Aspects}

The use of opium dates as far back as 3000 BC. It was extracted from the opium poppy by Sumerians in modern day Iraq, where it was probably used in religious ceremonies. Although opium use had reached Europe and Asia by the middle ages, China developed a severe opium addiction problem as a consequence of free opium trade (reviewed in Brownstein, 1993), enforced by the British during the opium war that spanned from 18401842 (Fay, 1975). The analgesic properties of morphine, the active ingredient of opium, were utilised after invention of the hypodermic syringe in the 1850 s. Ironically, the search for a non-addictive opiate compound that could be used as an analgesic resulted in the synthesis of diacetylmorphine, or heroin (reviewed in Pasternak, 2001).

At the beginning of the logarithmic expansion of knowledge on the endogenous opioid system and its pharmacology in the 1970s, a review written by Martin (1967) summarised the opioid field, outlining concepts such as adoption of the term 'opioid', opioid receptor agonism, partial agonism and antagonism and importantly, receptor dualism. Martin attempted to explain observations made with 'morphine-like agents' and 'nalorphine-like agents' by introducing the idea of multiple receptor populations. Pasternak and Snyder (1975) determined the existence of two types of opiate receptor, a high and a low affinity binding site, by using naloxone and dihydromorphine. Originally, it was proposed that 
these two binding sites, represented by biphasic binding to the "opiate receptor", were a result of different receptor conformations. However, less than 10 years after Martin (1967) had argued for the existence of multiple opioid receptors, the three classical opioid receptors, delta (DOR), kappa (KOR) and mu (MOR), were delineated using prototypic ligands and comparisons between different physiological systems (Snyder and Pasternak, 2003). MOR and KOR were originally defined in the chronic spinal dog (Martin et al., 1976). These receptors were named after their prototypic ligands, morphine and ketocyclazocine, respectively. Evidence for the existence of DOR came initially from the discovery that the mouse vas deferens requires 10 times more naloxone to inhibit enkephalin-induced contraction than what the mouse ileum required (Lord et al., 1977). It was proposed by Lord et al. (1977) that as binding profiles of specific opioids were different in different systems, opioid receptor sites must be heterogeneous, rather than just one homogenous population. The postulated existence of high affinity enkephalin binding sites (DOR) in the vas deferens was confirmed in later studies (Leslie and Kosterlitz, 1979).

The first endogenous opioid ligands to be characterised, the enkephalins, were extracted from crude brain homogenates (Terenius and Wahlstrom, 1975; Hughes, 1975; Hughes et al., 1975a; 1975b). These ligands were first recognised for their ability to block opiate receptor binding by dihydromorphine (Terenius and Wahlstrom, 1975). Thus, as the opioid system receptors were some of the first receptors in neuroscience to be characterised by ligand binding, the endogenous opioid ligands were some of the first neuropeptides to be characterised by receptor binding (Pasternak, 2004). 


\subsection{Molecular Biology and Biochemistry of the Opioid Receptors}

\subsubsection{Molecular Biology of the Opioid receptors}

The classical opioid receptor system now includes three cloned receptors. DOR was cloned first using functional screening with enkephalin (Evans et al., 1992; Kieffer et al., 1992). Isolation of cDNAs coding for KOR (Chen et al., 1993a; Li et al., 1993; Meng et al., 1993; Minami et al., 1993) and MOR (Chen et al., 1993b; Eppler et al., 1993; Thompson et al., 1993; Wang et al., 1993), using homologous probes based on DOR sequence, soon followed. Cloning the opioid receptors provided structural information that confirmed that they were 7-transmembrane domain G-protein coupled receptors. Homology models of the opioid receptors have been made utilising the closely related structure of the rhodopsin receptor (Subramanian et al., 2000; Fowler et al., 2004). Fowler et al. (2004) used homology modelling and mutagenesis techniques to determine important ligand binding residues for MOR (Fig. 1.1). The opioid receptors signal preferentially through an overlapping profile of pertussis toxin-sensitive G-proteins. This encompasses the $G_{i}$ and the $\mathrm{G}_{\mathrm{o}}$ alpha subunits (reviewed in Connor and Christie, 1999) and leads to affects such as inhibition of adenylyl cyclase and modulation of calcium signalling. Ligands that bind to DOR, KOR and MOR along with their affinities are listed in Table 1.1. 


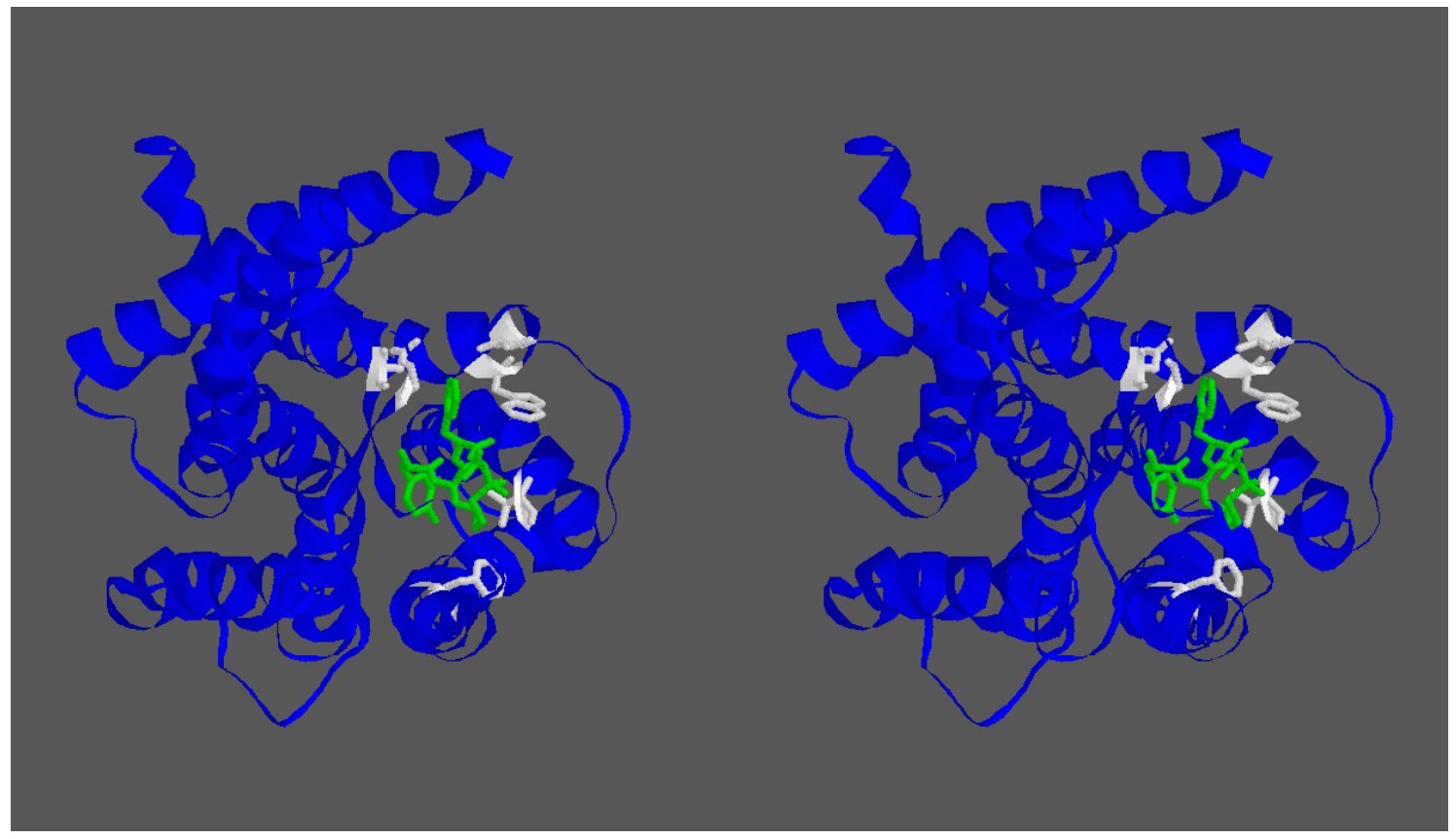

Fig. 1.1. A stereo-image of MOR, as seen from an extracellular view point. MOR (in blue) has 7 transmembrane alpha-helices. A cyclic peptide ligand, JOM6 (green), is seen bound within the ligand binding pocket where important binding residues described in Fowler et al. (2004) are shown in white. This image was generated using Rasmol v2.6 and a PDB file retrieved from http://mosberglab.phar.umich.edu/resources/index.php (Fowler et al., 2004).

\begin{tabular}{|c|c|c|c|c|}
\hline Ligand & $\begin{array}{l}\text { DOR } \\
(\mathrm{nM})\end{array}$ & $\begin{array}{l}\text { KOR } \\
(\mathrm{nM})\end{array}$ & $\begin{array}{l}\text { MOR } \\
(\mathrm{nM})\end{array}$ & Notes \\
\hline
\end{tabular}

\section{Exogenous agonists}

Morphine

Codeine

$\begin{array}{lll}160 & 47 & 1.8 \\ >1000 & 538 & 14\end{array}$

$>1000$
$>1000$
(Clark et al., 1988; 1989)

(Raynor et al., 1994)

Active ingredient in opium.

(Raynor et al., 1994)

Active ingredient in poppies 


\begin{tabular}{|c|c|c|c|c|}
\hline Fentanyl & $>1000$ & 255 & 0.39 & (Raynor et al., 1994) \\
\hline & & & & Common analgesic \\
\hline Methadone & $>1000$ & $>1000$ & 0.72 & (Raynor et al.,1994) \\
\hline PL017 & $>1000$ & $>1000$ & 30 & (Raynor et al., 1994) \\
\hline Etorphine & 0.4 & 0.8 & 2.0 & (Lewis and Husbands, 2004) \\
\hline Ethylketocyclazocine & 101 & 0.4 & 3.1 & (Raynor et al., 1994) \\
\hline U-50,488 & $>1000$ & 0.12 & $>1000$ & (Raynor et al., 1994) \\
\hline U-69,593 & $>1000$ & 0.59 & $>1000$ & (Raynor et al., 1994) \\
\hline DAMGO & $>1000$ & $>1000$ & 2.0 & $\begin{array}{l}\text { (Raynor et al., 1994) Synthetic } \\
\text { enkephalin analogue }\end{array}$ \\
\hline DPDPE & 14 & $>1000$ & $>1000$ & $\begin{array}{l}\text { (Raynor et al., 1994) Synthetic } \\
\text { enkephalin analogue }\end{array}$ \\
\hline DSLET & 4.8 & $>1000$ & 39 & $\begin{array}{l}\text { (Raynor et al., 1994) Synthetic } \\
\text { enkephalin analogue }\end{array}$ \\
\hline
\end{tabular}

\section{Exogenous antagonists}

$\begin{array}{lllll}\begin{array}{l}\text { (-)-Naloxone } \\ \text { Naltrexone }\end{array} & 17 & 2.3 & 0.93 & \text { (Raynor } \text { et al., 1994) } \\ \begin{array}{l}\text { Naloxonazine } \\ \text { Nalorphine }\end{array} & 149 & 3.9 & 1.0 & \text { (Raynor } \text { et al., 1994) } \\ \text { CTOP } & 14.6 & 11 & 0.054 & \text { (Raynor } \text { et al., 1994) } \\ \text { Beta-FNA } & >1000 & >1000 & 0.18 & \text { (Raynor } \text { et al., 1994) } \\ \text { Norbinaltorphamine } & 48 & 2.8 & 0.33 & \text { (Raynor } \text { et al., 1994) } \\ & 65 & 0.027 & 2.2 & \text { (Raynor } \text { et al., 1994) }\end{array}$

\section{Endogenous peptide ligands}




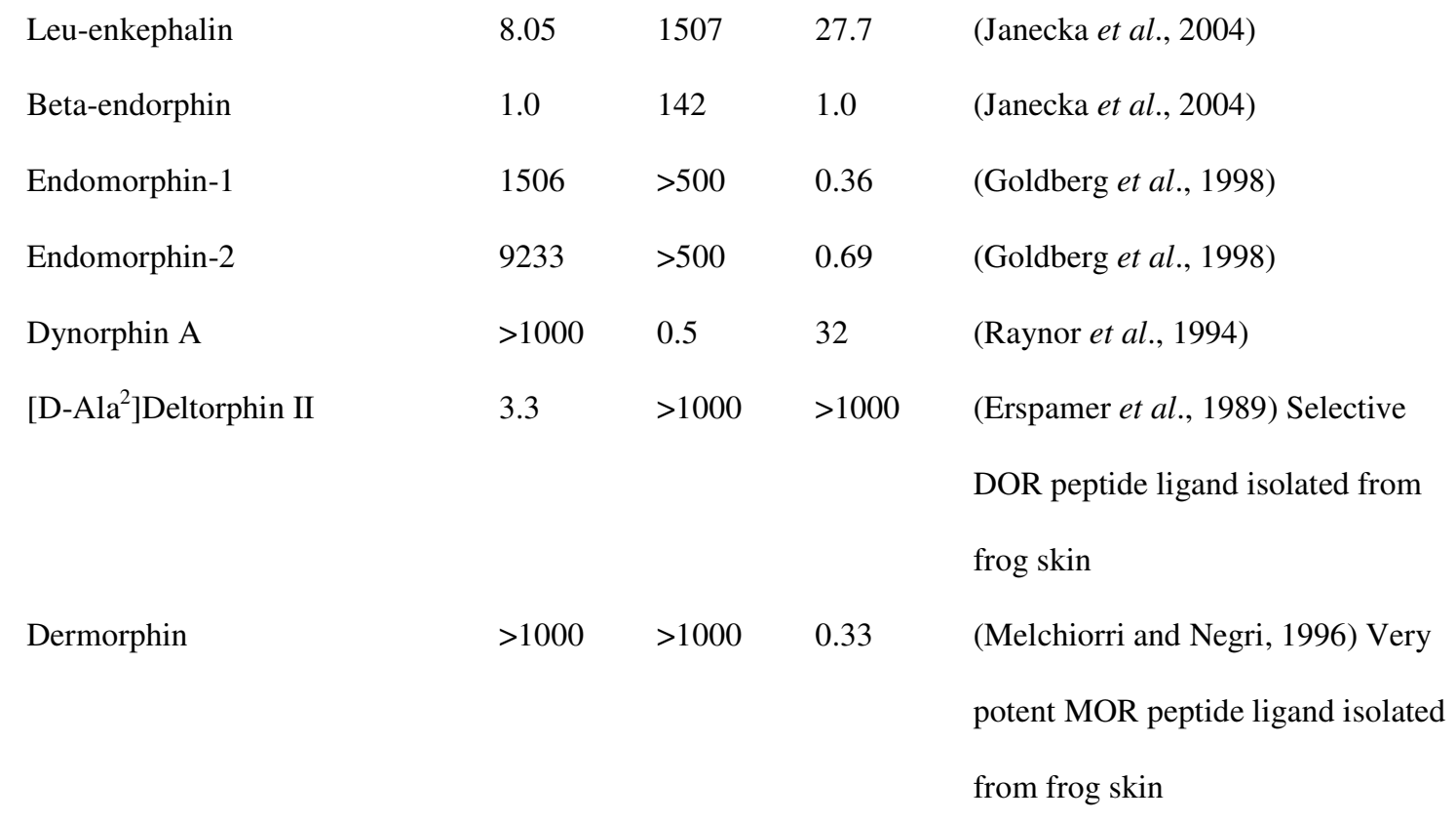

Table 1.1: Opioid receptor ligands. This table shows common opioid receptor ligands shown with $\mathrm{K}_{\mathrm{i}}$ values against receptor-selective ligands taken from the literature. Raynor et al. (1994) used tritiated U69,593 (KOR), naltrindole (DOR) and DAMGO (MOR) for determination of Ki values.

To date, only three opioid receptors have been cloned; DOR, KOR and MOR. Two other opioid related receptors have also been cloned. The opioid receptor-like 1 (ORL1) receptor bears high homology to the opioid receptors (in particular KOR), to the extent it was originally identified by screens based on opioid receptor nucleotide sequence. Its ligand, nociceptin also bears striking resemblance to dynorphin, the endogenous KOR ligand. In vivo, the ORL1 receptor modulates anti-opioid affects (reviewed in Meunier, 1997). Another receptor, the opioid growth factor receptor (OGFr), was cloned and identified using expression libraries and antibody raised against opioid growth factor (metenkephalin) binding proteins that were identified on a 2-dimensional gel (Zagon et al., 1999a). 
Although only one MOR gene (Oprm1) has been cloned, a multitude of MOR splice variants have been discovered, most notably in the mouse (reviewed in Pan, 2005). MOR splice variants have also been described in the rat (Zimprich et al., 1995; Pasternak et al., 2004) as well as in the human (Bare et al., 1994; Pan et al., 2003; Pan et al., 2005a). Alternative splicing is an important step in introducing increased complexity (thus increased control) in gene expression. Alternative splicing is a process that wields great influence over brain development, as seen with alternative splicing of fibroblast growth factor and numb gene products (reviewed in Li et al., 2007). At least 25 MOR splice variants have been isolated from the mouse (encompassing MOR-1, MOR-1A through MOR-1T) (Pan, 2005). The different MOR carboxy-terminus splice variants also show different ligand binding and receptor stimulation profiles (Bolan et al., 2004). Interestingly, both carboxy as well as amino terminus splice variants of MOR differ in terms of immunohistochemical localisation within the adult rodent brain (Abbadie et al., 2000; Abbadie et al., 2004).

The numerous splice variants are driven from two promoters. The first one that was discovered was the promoter region immediately upstream of exon 1 of the Oprm 1 gene (Min et al., 1994; Liang et al., 1995). This murine MOR promoter region contains two promoter sites (Liang et al., 1995), one proximal site at 268 base pairs upstream of the exon 1 translation start point and another distal transcription start point at 793 base pairs upstream of the translation start point. This distal transcription start point is of particular interest as it is under the positive control of sox6, sox 18 and sox21 (Hwang et al., 2003). Sox transcription factors are important for developmental processes (Wegner and Stolt, 
2005). Sox6 is neurogenic in that it is required for the retinoic acid induced neuronal differentiation of P19 embryonal carcinoma cells (Hamada-Kanazawa et al., 2004a; 2004b) and that over expression of sox 6 is sufficient to induce neurogenesis by itself. Sox 21 is also neurogenic such that overexpression causes cells within the developing central nervous system (CNS) to exit the cell cycle and become neurons (Sandberg et al., 2005). This is interesting given that MOR also has pro-neurogenic properties (Kim et al., 2006) and that expression of murine MOR transcript from the distal promoter site begins at embryonic day (E)8.5 (Ko et al., 2002), at the very beginning of neurogenesis in the developing mouse CNS (reviewed in Götz and Huttner, 2005).

The second major promoter that drives transcription from exon 11 was discovered and analysed when exon 11-containing splice variants were cloned (Pan, 2002). These splice variants contained exons 5 ' of exon 1 , thus they were the product of promoter activity upstream of the exon 1 promoter region (Pan et al., 2001). Evidence for alternative splicing of DOR and KOR transcripts remains to be presented. The exon 1 and exon $11 \mathrm{MOR}$ promoters are subject to differential developmental regulation (Xu et al., 2006). Exon 1 promoter activity has been reported in the E7.5 mouse brain whereas in the same study, exon 11 promoter activity was detected later at E11.5.

\subsubsection{Phamacological Variants of the Opioid Receptors}

Although only three classical opioid receptors have been cloned, several pharmacological subtypes of the opioid receptors have been proposed. Two pharmacological MOR binding 
sites were characterised using naloxazone. These binding sites were the high affinity $\mathrm{mu}_{1}$ $\left(\mathrm{K}_{\mathrm{d}}<1 \mathrm{nM}\right)$ and the lower affinity $\mathrm{mu}_{2}\left(\mathrm{~K}_{\mathrm{d}}>3 \mathrm{nM}\right)$ (Wolozin and Pasternak, 1981). It was found that naloxazone, as well as naloxonazine (Hahn and Pasternak, 1982) abolished binding to the high affinity $\mathrm{mu}_{1}$ binding sites.

Naloxazone and naloxonazine $\left(\mathrm{mu}_{1}\right.$ receptor antagonists with no antagonistic properties at the $\mathrm{mu}_{2}$ receptor binding site) were used to determine the pharmacological significance of the identified $\mathrm{mu}_{1}$ and $\mathrm{mu}_{2}$ binding sites. Whereas beta-funaltrexamine (beta-FNA) universally antagonised morphine's effects in vivo, naloxonazine and naloxazone were much more selective. Naloxazone was used to demonstrate that prolactin release, but not growth hormone release, was dependent on $\mathrm{mu}_{1}$ (Spiegel et al., 1982). Other studies have shown that $\mathrm{mu}_{1}$ binding sites are critical for morphine induced catalepsy (Ling and Pasternak, 1982). Morphine induced analgesia is also modulated by pharmacological MOR variants. Both $\mathrm{mu}_{1}$ and $\mathrm{mu}_{2}$ binding sites are involved with $\mathrm{MOR}$ induced analgesia depending on whether the analgesia is spinal or supraspinal (Ling and Pasternak, 1983; Paul et al., 1989). Furthermore, studies have demonstrated that pharmacological properties of morphine can be dissociated using $\mathrm{mu}_{1}$ specific antagonists. Such studies have demonstrated the separation of morphine induced analgesia and respiratory depression (Ling et al., 1983; 1985), morphine induced analgesia and dependence and withdrawal (Ling et al., 1984), as well as separation of opioid peptide mediated analgesia and opioidergic modulation of peristalsis (Heyman et al., 1988). The activity of $\mathrm{mu}_{1}$ and $\mathrm{mu}_{2}$ binding sites in modulation of respiration was further delineated by Colman and Miller (2001) who demonstrated the involvement of both mu subtypes in respiratory depression 
using the MOR agonists fentanyl and dermorphin. Another line of evidence that has been used to support the existence of multiple mu binding sites is mu opioid synergy. Bolan et al. (2002) demonstrated that L-methadone displays synergy with some mu opioids such as morphine, morphine-6-beta-glucuronide, 6-acetylmorphine and codeine, but not others such as fentanyl and meperidine.

An attempt to reconcile the now copious evidence for both MOR splice variants and the pharmacological MOR variants, $\mathrm{mu}_{1}$ and $\mathrm{mu}_{2}$, argues that mu opioid ligand activity is a summation of action across all splice variants (Pasternak, 2004). Thus different pharmacological activities given by different mu opioids exist as different mu opioids stimulate different MOR splice variants to varying extents. As it has also been shown that different MOR splice variants have differing localisations, it is not hard to imagine how specific mu opioid ligand-MOR splice variant pairs could generate unique pharmacological effects. This goes a long way in reconciling pharmacological classification with the known molecular biology of opioid receptors.

DOR and KOR also have reported pharmacological variants. Evidence for DOR pharmacological variants came in the form of a lack of antinociceptive cross-tolerance to the highly selective delta opioid agonists [D-pen $\left.{ }^{2}, \mathrm{D}-\mathrm{pen}^{5}\right]$ enkephalin (DPDPE) and deltorphin II (Mattia et al., 1991). DOR mediated antinociception by different DOR selective ligands was also sensitive to different opioid antagonists (Jiang et al., 1991). Further characterisation of DOR subtypes was conducted by Xu et al. (1991) who also provided evidence for two DOR binding sites. 
The kappa $a_{1}$ and kappa $a_{2}$ binding sites were differentiated based on high and low binding affinity (respectively) to KOR ligands such as U69,593, U50,488 and bremazocine (Zukin et al., 1988). Subsequently, evidence was presented for the existence of a third kappa opioid binding site, kappa 3 , using the high affinity ligand naloxone benzoylhydrazone (Clark et al., 1989). Another study resolved these three kappa opioid binding sites into four pharmacological variants (Rothman et al., 1990).

The existence of multiple pharmacological variants of the opioid receptors has attracted criticism. Naloxone benzoylhydrazone was recognised as the prototypic kappa 3 agonist. In a recent study, it was shown that naloxone benzoylhydrazone was a partial agonist at all three cloned classical opioid receptors, DOR, KOR and MOR, and a pure antagonist at ORL1 (Olianas et al., 2006). Connor and Kitchen (2006) use these data, as well as the fact that none of the proposed kappa pharmacological subtypes have ever been cloned, to argue that the idea of multiple opioid receptors, KOR in particular, should be put to rest until more sound supporting data comes to light.

\subsubsection{Homo- and Heterodimerisation of the Opioid Receptors}

Adding further complexity to the opioid field, opioid receptor homo and heterodimerisation has now been characterised. DORs homodimerise in an agonist-dependent manner. DOR dimers dissociate to monomers in the presence of increasing DOR agonist, such as [D- 
$\mathrm{Ala}^{2}, \mathrm{D}-\mathrm{Leu}^{5}$ ]enkephalin (DADLE). This event preceded internalisation, suggesting a relationship between dimerisation and internalisation (Cvejic and Devi, 1997).

Interestingly, in the same study, the agonist morphine did not produce the same dissociation effect. Morphine has also been noted for its unique inability to induce opioid receptor internalisation amongst opioid receptor ligands. Arden et al. (1995) demonstrated that morphine could not induce receptor internalisation even though [D-Ala $2, \mathrm{NMePhe}_{4}$, Glyol]enkephalin (DAMGO, a selective MOR agonist) could. Morphine is also unable to internalise DOR, at concentrations that lead to receptor activation (Keith et al., 1996). Wang et al. (2005), using quantitative bioluminescence resonance energy transfer, provided supporting evidence for the idea that all opioid receptors have the ability to homodimerise.

A substantial amount of data shows that MOR also heterodimerises with DOR. Heterodimerisation of MOR and DOR was demonstrated using immunoprecipitation studies as well as bioluminescence resonance energy transfer (George et al., 2000; Gomes et al., 2000; 2004; Law et al., 2005; Wang et al., 2005; Rozenfeld and Devi, 2007). Several studies have shown that DOR-MOR heterodimerisation has distinct functional consequences. Unlike DOR-DOR homodimers, agonist treatment increases the binding of DOR and MOR agonists, such as deltorphin II and [D-Ala ${ }^{2}, \mathrm{~N}-\mathrm{Me}-\mathrm{Phe}^{4}, \mathrm{Gly}^{5}$-ol $]$ enkephalin (DAMGO) to the DOR-MOR heterodimer, and these ligands display synergy at heterodimer sites (Gomes et al., 2000). Evidence for in vivo heterodimerisation has also been presented. DOR-MOR heterodimers have been isolated from spinal cord membranes of the mouse. In line with this, it has been shown that DOR antagonism greatly enhances MOR induced analgesia (Gomes et al., 2004). Rozenfeld and Devi (2007) demonstrated 
that heterodimerisation affects opioid receptor signalling through extracellular-signal regulated kinase (ERK). ERK phosphorylation occurs rapidly (within 5 mins) upon opioid agonist treatment. The opioid receptor monomer is responsible for this rapid activation. However, the DOR-MOR heterodimer induces a robust second phase of ERK phosphorylation, not seen when the opioid receptor dimer is not present. MOR (Wang et al., 2005) as well as DOR (Jordan and Devi, 1999) has also been shown to heterodimerise with KOR in transfected cell studies.

Opioid receptor pharmacology extends beyond dimerisation between the classical opioid receptors. MOR can also heterodimerise with ORL1 (Wang et al., 2005). Furthermore, this interaction desensitises MOR signalling and is suggested to contribute towards the antiopioid effects of the ORL1 receptor. Significant pharmacological interaction seen between MOR and cannabinoid signalling may also be, in part, a result of receptor heterodimerisation. Using bioluminescence resonance energy transfer, Rios et al. (2006) demonstrated close physical interaction between MOR and cannabinoid-1 receptors.

Opioid receptor signalling at the level of the opioid receptor is complicated by multiple ligands, receptor variants and receptor interaction. Endogenous as well as exogenous ligands display promiscuity with multiple receptor subtypes. Pharmacological opioid receptor variants have not been reconciled with the molecular characterisation of multiple MOR splice variants and remain unsubstantiated by molecular data. Furthermore, recent evidence shows that opioid receptors may signal via both homo- and heterodimers. 


\subsection{Physiological Roles of the Opioid System}

Opioid compounds have been used extensively as powerful analgesics. MOR is responsible for opiate mediated analgesia, however KOR and DOR also play more subtle, complicated roles in pain perception. MOR mediated analgesia occurs directly at the spinal level (in the sensory dorsal horns) but also acts at supraspinal sites that are involved in pain such as the amygdala, insular cortex, hypothalamus, periaqueductal grey and the medulla. At a cellular level, MOR accomplishes these effects by inhibition of inhibitory gamma amino butyric acid (GABA) release to activate neurons that inhibit pain or by direct inhibition on neurons that cause pain by activating G-protein coupled inwardly rectifying potassium channels (reviewed in Fields, 2004).

MOR has been implicated in reward and addiction in the ventral tegmental area-nucleus accumbens pathway. MOR stimulation in the ventral tegmental area dis-inhibits dopaminergic neurons that project into the nucleus accumbens by inhibition of GABAergic neurons. Increases in cAMP levels and cAMP response element binding protein (CREB) associated with chronic opiate use in the nucleus accumbens is also linked to tolerance and dependence. KOR works in opposition to MOR to inhibit the ventral tegmental areanucleus accumbens pathway (reviewed in Nestler, 2004).

Other physiological roles that the opioid system regulates include reward-related feeding (reviewed in Levine and Billington, 2004), gastrointestinal transit (reviewed in Bohn and Raehal, 2006), respiratory depression (Colman and Miller, 2001) and sex drive (reviewed 
in Hull and Dominguez, 2007). Pharmacological effects of the opioid system have been verified using opioid receptor knockout animals (Tian et al., 1997; reviewed in Kieffer and Gaveriaux-Ruff, 2002). Interestingly, MOR knockout (KO) mice displayed defective maternal attachment behavior such that mouse pups failed to display signs of distress vocalisation when separated from their mothers (Moles et al., 2004).

Importantly, opioids also modulate the immune system. Opioids are immunosuppressive in that they alter cytosolic activity and antibody production in immunocytes as well as suppressing proliferation of lymphocytes, possibly by direct activation of opioid receptors expressed by these cells (reviewed in Wei et al., 2003). In support for a role of the opioid receptor system in proliferation in the immune system, homozygous disruption of MOR resulted in higher rates of proliferation in myeloid progenitor cells taken from the spleen and bone marrow (Tian et al., 1997).

The opioid system has a broad range of physiological roles, the most well known of which are in analgesia and reward. KO studies of the opioid receptors have yielded supporting evidence for these roles, including modulation of proliferation in the immune system. Another proposed role for the opioid receptor system is for modulation of proliferation in the CNS. 


\subsection{The Opioid System and Proliferation within the CNS}

\subsubsection{Opioid Signalling System Expression in the Foetal Brain}

\subsubsection{Opioid Receptor Ligand Expression}

Although very few studies have directly investigated opioid signaling and opioid/opioid receptor gene expression at the time of neurogenesis in the foetal brain, there is a growing body of evidence in the literature that demonstrates all elements of the opioidergic signaling system are expressed within the developing foetal brain. Studies that investigated the presence of the opioid peptide precursors and their cognate peptidases have made use of mRNA in situ hybridisation and immunohistochemistry. Data from the literature showed that endogenous opioid peptides, including the enkephalins, dynorphin, and betaendorphin, can be found in the foetal rodent brain.

Both met- and leu-enkephalin have been isolated from E13-E16 rat brain homogenates by HPLC (Dahl et al., 1982). Extensive proenkephalin mRNA expression has been shown in the ventricular zone (VZ) of the lateral ventricle (LV) in the E15 and E21 rat (Leslie et al., 1998). This study showed that proenkephalin expression was also exclusive to the neuroepithelia of the telencephalon. Proenkephalin expression in the rat VZ, however, was biphasic such that early in corticogenesis (E13), proenkephalin mRNA expression was relatively high, whereas expression dropped to nearly nothing at E17 only to peak again at 
birth (E22). In another study by Zagon et al. (1994), proenkephalin mRNA was intensely expressed in the E18 choroid plexus, although in contrast to Leslie et al. (1998), this study reported widespread expression throughout the brain as well.

Several studies have looked at proenkephalin expression in the developing cerebellum with particular reference to the external granule layer (EGL), a secondary proliferative zone. Proenkephalin mRNA as well as enkephalin protein expression has been reported in the rat cerebellum from late gestation to adulthood (Zagon et al., 1985; 1994). Early in development, proenkephalin mRNA was expressed in the EGL. In the adult cerebellum, this expression was limited to the Purkinje cells and Golgi II cells (Zagon et al., 1994). In contrast, proenkephalin mRNA was not found in cells of the EGL by Osborne et al. (1991; 1993). Interestingly, small amounts of enkephalin peptide were detected in the EGL in the same study (Osborne et al., 1993).

Data on the localisation of beta-endorphin expression within foetal neuroepithelia have yet to be presented. However, the ontogeny of beta-endorphin within the hypothalamus and the pituitary gland has been described. The appearance of proopiomelanocortin (the precursor protein to beta-endorphin) immunoreactivity (IR) appears in the rat hypothalamus at E12. Proopiomelanocortin IR in the pituitary gland appears at E15 (Khachaturian et al., 1983). Interestingly, proopiomelanocortin mRNA expression was higher in the E20 rat hypothalamus than in the postnatal rat hypothalamus (Angelogianni et al., 2000). The increased expression of proopiomelanocortin mRNA before birth, as opposed to after birth, is mirrored in results by Rius et al. (1991) who showed that beta-endorphin was detectable 
in whole brain homogenates from E11.5 onwards and that these levels peak just before birth in the mouse, only to drop off dramatically just afterwards. Beta-endorphin peptide remained low even at postnatal day $(\mathrm{P}) 3$. Expression of prodynorphin mRNA, which codes for KOR peptide-ligand, dynorphin $\mathrm{A}$, is abundant in rat hindbrain neuroepithelia, peaking at E15 and dropping to nothing at birth (Leslie et al., 1998). Rius et al. (1991) found that dynorphin A was expressed from E11.5, but whole mouse foetal brain homogenates exhibited lower amounts of dynorphin A than enkephalin or beta-endorphin peptides. Dynorphin A levels in the whole foetal brain were 100 times lower than in the whole adult brain. In contrast to the other opioid peptides, the specific, high affinity MOR ligands, endomorphin-1 and endomorphin-2 were not expressed at all until P7 in the rat brain (Barr and Zadina, 1999); however, met- and leu-enkephalin, which are expressed prenatally, have high affinities for MOR.

Further support for a developmental role for the opioid system has come from investigation of cell surface peptidases that degrade opioid peptides. Endopeptidase 24.11 (enkephalinase) is known to cleave and inactivate enkephalins, and also has the potential to cleave dynorphin and the endorphins (reviewed in Erdos and Skidgel, 1989). Enkephalins are also degraded by aminopeptidase $\mathrm{N}$ (reviewed in Luan and $\mathrm{Xu}, 2007$ ). Interestingly, the choroid plexus of the developing rat brain (E14 onwards) strongly expresses enkephalinase at the mRNA level (Dutriez et al., 1992; Spencer-Dene et al., 1994) and protein level (Spencer-Dene et al., 1994). Enkephalinase IR has also been shown in the ependymal linings of the rat brain ventricular system from E14 onward. Inhibition of enkephalinase in the whole rat embryo at E9.5 causes gross but asymmetric overgrowth of the 
prosencephalic neuroepithelia (Spencer-Dene et al., 1994). Thus although enkephalinase does have other substrates, such as substance $\mathrm{P}$, the localisation of a major component of the opioid system in structures such as the choroid plexus, as well as the VZ of the ventricular system further implicates opioid system signaling in development of the brain.

\subsubsection{Opioid Receptor Expression}

Expression of the opioid receptors occurs from their TATA-less promoters (with the exception of the mouse exon 11 promoter, which does contain a TATA box (Pan, 2002)) and is regulated by numerous transcription factors, indicating complex control over gene transcription (Wei and Loh, 2002). The earliest ontological studies that assessed opioid receptor radioligand binding in rodent foetal brain homogenates found that $\left[{ }^{3} \mathrm{H}\right]$ naloxone (Coyle and Pert, 1976) and $\left[{ }^{3} \mathrm{H}\right]$ naltrexone (Clendeninn et al., 1976) binding increased from E14 until adulthood. Early in situ studies using radioligand binding for opioid receptors in the developing rodent foetus claimed that opioid binding sites within the rat striatal subventricular zone (SVZ) first appeared around E14 (Kent et al., 1982, reviewed in Leslie and Laughlin, 1993). At E16 (at the peak of cortical neurogenesis in the rat), Kent et al. (1982) reported opioid receptor binding in the rat SVZ. This ligand binding extended further down into the rat striatal SVZ toward the ventricular wall until E20, when it reached the ventricular edge.

In contrast to later studies that failed to identify opioid receptor mRNA in the developing cerebellum, early studies also found opioid binding sites within the cerebellum. Etorphine 
bound to opioid receptors in cerebellar homogenates (Meunier and Zajac, 1979). Binding was greatest in molecular layer homogenates although some binding was seen in granular layer and white matter cerebellar homogenates of rabbits. DOR, KOR and MOR binding sites have been reported in rat and guinea-pig cerebellar homogenates. The density of opioid receptor binding sites declines to a low level at adulthood (Barg and Simantov, 1989).

Some studies that have looked at mRNA distribution in the developing embryo have completely discounted the expression of opioid receptors in the $\mathrm{VZ}$ of the developing brain. Radio in situ hybridisation studies showed no expression of opioid receptors in the proliferative regions of the embryonic mouse and rat CNS (Leslie et al., 1998; Zhu et al., 1998). In contrast, other in situ hybridisation studies found MOR transcript in the rat striatal neuroepithelium from E13 onwards (Georges et al., 1998) as well as in the E16 rat EGL of the cerebellum (Mrkusich et al., 2004). These findings were consistent with earlier ligand binding studies. Tong et al. (2000) also detected MOR mRNA expression in germinal zones of the E12 rat brain. An immunohistochemical study of the embryonic mouse brain has found evidence of DOR, KOR and MOR expression in the VZ of the dorsal telencephalon (Reznikov et al., 1999).

\subsubsection{Opioid Signaling System KOs}

Although the endogenous opioid system is suggested to play a role in development, $\mathrm{KO}$ of elements of the opioid system produces a remarkable lack of developmental phenotypes 
(reviewed in Gaveriaux-Ruff and Kieffer, 2002 and Kieffer and Gaveriaux-Ruff, 2002). Single opioid receptor KOs and even combined opioid receptor KO mice appear healthy and, apart from insensitivity to opioid related responses, relatively normal, indicating no major developmental pathologies.

Investigation of developmental processes by way of gene $\mathrm{KO}$ has not always provided a faithful means of identifying gene function. $\mathrm{KO}$ of doublecortin (DCX) (a causative gene in type I lissencephaly in humans) in the mouse resulted in normal cortical laminar architecture (Corbo et al., 2002). Only when DCX was knocked down via RNA interference in the developing rat brain was the human pathology recapitulated (subcortical band heterotopias and ectopically positioned neurons) (Bai et al., 2003). The authors of this study reconcile their results with the DCX KO study (Corbo et al., 2002) by suggesting that gene $\mathrm{KO}$ from conception allows the developing system time to implement compensatory measures. Also, Bai et al. (2003) state that as knockdown is not $100 \%$ effective, some cells within the same system will express the target gene at wild type levels. A similar observation was reported by Mizutani and Gaiano (2006) who noted that whereas the Foxg1 heterozygote KO had no reported developmental defect, an RNA interference knockdown of Foxg1 to 50\% in cortical progenitor cells produced an observable phenotype. Mizutani and Gaiano (2006) also speculated that compensatory measures taken by the $\mathrm{KO}$ animal may have accounted for this discrepancy. Opioid receptor knockdown experiments have been conducted have investigated opioidergic modulation of antinociception in the adult rodent CNS (Bilsky et al., 1996; Wang et al., 1996). The closest a developmental study has come to acute disruption of the endogenous opioid 
signaling system (without introducing exogenous opioid receptor ligands) was the pharmacological inhibition of enkephalinase by Spencer-Dene et al. (1994), which did result in developmental disruption of the brain.

\subsubsection{Opiates and Development}

\subsubsection{Opiates and Growth of the Developing Rodent}

Early studies using methadone (a MOR agonist used as a narcotic substitute) showed no gross teratogenicity with rabbits and rats. It was noted that animals being treated with methadone ate less and gained less weight during pregnancy (Markham et al., 1971). The same study concluded that methadone was not teratogenic. The apparent lack of teratogenicity of methadone was an observation that was backed up by Chandler et al. (1975). Chandler et al. (1975) also noted that pups that were dissected out of the mother just before birth were lighter than control animals, an observation that was corroborated by Hutchings et al. (1976). Buchenauer et al. (1974) reported increased pup mortality and pup growth retardation in methadone treated pregnant rats; however, the same study also failed to produce any truly teratogenic effects. Methadone treatment over E8-E22 in the rat has been shown to reduce birth weight in a dose-dependent manner (Hutchings et al., 1976). However, by the time of weaning, methadone-induced weight effects had all but disappeared. Hutchings et al. (1976) also showed that the opiate methadone readily crosses the placenta to reach the foetus. Methadone administration to rats before, during and after 
gestation results in lowered RNA and protein content in the brains of the offspring (Peters, 1977). Peters (1977) also reported lowered DNA content in the brain; however, they also noted that these data were in line with a general reduction in body weight. Zagon and McLaughlin (1977b) also reported a methadone induced reduction in body size of the pup and a reduction in brain DNA content. As well as for methadone, a reduction in rat pup body weight during postnatal development was seen with chronic morphine treatment over gestation (Tao et al., 2001).

A rigorous study by Hutchings et al. (1992) used osmotic minipumps to administer methadone to pregnant rats from E8 until the end of gestation. This study found that gradual osmotic release of methadone produced greatly reduced maternal and foetal toxicity as compared to equivalent doses of injected methadone in the rat. Hutchings et al. (1992) did not see any difference in birth-weights for control or methadone treated animals. However, they did report a transitory deficit in growth for high dose methadone pups (15 $\mathrm{mg} / \mathrm{kg} /$ day). Interestingly this same group displayed altered rest patterns similar to what has been observed in studies that use injections as opposed to osmotic pumps.

In contrast to the results of Hutchings et al. (1976), another study reported lasting weight changes in animals exposed to methadone throughout gestation and lactation (Zagon and McLaughlin, 1977a). The opioid antagonist, naltrexone (50 mg/kg/day) significantly enhanced development in neonatal rats (P0 to P21) (Zagon and McLaughlin, 1983) as naltrexone was shown to enlarge bodyweight as well as the size of the brain. Overall, 
animal studies that investigate the effect of opiates on growth of the foetus and pup demonstrate mild negative effects that are dependent on dose and mode of delivery.

\subsubsection{Human Development and In Utero Opiate Exposure}

Opiate drugs are liable to abuse by humans due to their ability to elicit feelings of euphoria and extremely high addictive potential. A significant proportion of people who abuse opiates are women of child bearing age. Of the studies that analysed the effect of in utero opiate exposure on the developing child, early studies demonstrated effects on intellectual performance and motor development, as well as behavioural problems (Wilson et al., 1981; Rosen and Johnson, 1982; Bunikowski et al., 1998). However, it is now recognised that confounding variables such as poly-drug exposure, socio-economic status, parental care and small sample sizes were responsible for the early observed effects (Lester et al., 2001). Other studies have also noted that detrimental developmental effects associated with in utero opiate exposure are more likely a result of poor environmental conditions (Lifschitz et al., 1985; Ornoy et al., 1996; Hans and Jeremy, 2001; Lester et al., 2002; Messinger et al., 2004). It has been observed that along with opiates, people also abuse cocaine, marijuana, tobacco and alcohol (Lester et al., 2001; Lejeune et al., 2006), all of which are harmful to the developing foetus.

Even after collection of sufficiently large sample sizes and consideration of detrimental covariates such as socio-economic status and poly-drug abuse, in utero opiate exposure still has subtle effects on the developing child. After extensive adjustment for co-variates, in 
utero opiate exposed infants ( 1 month old) have fewer short utterances and had more hyperphonation (very high pitched cries) (Lester et al., 2002). Acoustic parameters of an infant's cry gives information on the biological integrity of an infant. Infants that have suffered brain damage of various causes have higher pitch cries. In infants that have suffered neurological damage, a higher cry pitch was correlated with a poorer neurodevelopmental outcome (Corwin et al., 1996). Infants of opiate abusing mothers also displayed longer latencies to wave V on auditory brain stem tests (Lester et al., 2003). This represents a slowing of nervous conduction from the cochlea to the inferior colliculus.

\subsubsection{Opiates and Cellular Proliferation in the CNS}

The effects of opiates on growth, illustrated extensively in animal studies using methadone, are largely explained in the literature by an inhibitory effect on cellular proliferation. The characterisation of opioidergic modulation of cellular proliferation has culminated recently with the finding that opioid receptor signalling is involved in embryonic stem cell proliferation and differentiation. Kim et al. (2006) place MOR and KOR expression on pluripotent stem cells at embryonic day 3.5, albeit in cell culture. At mitosis, the stem cells switched from a symmetric, self-renewing division to an asymmetric division that yielded nestin and sox-1-positive neuronal progenitors. Kim et al. (2006) also showed that retinoic acid-differentiated embryonic stem cell (neuronal progenitor cell) proliferation was inhibited by kappa and mu opioid signalling. KOR stimulation also increased proliferation in nestin-positive neural precursor cells isolated from $\sim \mathrm{E} 42$ human embryonic tissue (Sheng et al., 2007). Interestingly, when murine retinoic acid-derived neuronal progenitor 
cells were exposed to the same kappa and mu opioid agonists, an antiproliferative effect was observed (Kim et al., 2006).

\subsubsection{Opiates and Proliferation in the Rodent Forebrain}

Studies have shown that opioids impact upon proliferation in the developing forebrain, although few of these studies detail these effects in the foetal forebrain. DOR mediates proneuronal differentiation in the MEB5 cell line (multipotent stem cells taken from E14.5 forebrain). These cells differentiate to form neurons when exposed to DOR ligand SNC80 (Narita et al., 2006). In the same study, the selective MOR ligand, DAMGO and the KOR selective ligand, U50,488H did not affect proliferation or differentiation. This study contrasts with other studies that have found kappa and mu to be responsible for opioid mediated effects on proliferation (Barg et al., 1993; Knapp et al., 1998; Kim et al., 2006; Sheng et al., 2007).

Reznikov et al. (1999) showed that MOR stimulation by DAMGO in utero modulated the cell cycle within the cortical ventricular neuroepithelium of the E16 mouse (labelled E15 in our study). The authors of this study showed that acute DAMGO and bremazocine (KOR agonist) exposure increased the number of S-phase cells (cells that incorporated $\left[{ }^{3} \mathrm{H}\right]$ thymidine) as well as the number of mitotic nuclei. The authors of this study interpreted these data as an increased duration of both $\mathrm{S}$ phase and mitosis of proliferative cells. In the same study, [D-ser $\left.{ }^{8}-\mathrm{leu}\right]$ enkephalin[Thr] (DSLET) (a DOR agonist) reduced the number of $\left[{ }^{3} \mathrm{H}\right]$ thymidine labelled nuclei and mitotic nuclei. 
Unfortunately, Reznikov et al. (1999) indicated that they used only one pregnant dam per experimental group. The use of multiple littermates in one experimental group to inflate the $\mathrm{n}$ value leaves the study susceptible to confounding litter effects (discussed further in Chapter 2) (Holson and Pearce, 1992; Zorrilla, 1997). Interpretation of the study by Reznikov et al. (1999) must be approached with caution.

In the postnatal forebrain, morphine decreased $\left[{ }^{3} \mathrm{H}\right]$ thymidine incorporation into the rat brain at P1 and P4. Older rats (up until P12) did not show this effect (Kornblum et al., 1987). Morphine treatment (from E12-P6) has also been shown to modulate corticogenesis in vivo in the developing rat by reducing neuronal density of the somatosensory cortex without affecting its thickness (Seatriz and Hammer, 1993). Interestingly, in the same study, naltrexone administration did not increase numbers of neurons. Beta-endorphin produced a robust decrease in $\left[{ }^{3} \mathrm{H}\right]$ thymidine incorporation in the brain that persisted for 11 $\mathrm{h}$ in the P20 rat (Bartolome et al., 1991). This affect was successfully antagonized by naloxone. Lorber et al. (1990) demonstrated that proliferation in the postnatal brain showed a developmentally sensitive response to beta-endorphin mediated inhibition with maximal effect observed during P4-P8. Beta-endorphin mediated inhibition of DNA synthesis in the pre-weanling rat brain was also antagonized by a sulfated fragment of cholecystokinin (Bartolome et al., 1994). DNA synthesis in the P11 forebrain also decreased in response to $\left[\mathrm{D}-\mathrm{Met}^{2}-\mathrm{Pro}^{5]}\right.$ enkephalinamide (Vertes et al., 1982). 
Opioid antagonism also has effects on the postnatal forebrain. Naloxone shows biphasic effects on DNA synthesis in the P11 rat brain (Vertes et al., 1982). Incorporation of $\left[{ }^{3} \mathrm{H}\right]$ thymidine in the forebrain increased in response to naloxone treatment up until $3 \mathrm{~h}$ post-treatment. Proliferation then dropped dramatically lower than in control animals. In addition, it was shown that the opiate antagonist, naltrexone, produced a long-lasting increase in cellular proliferation in the 4-12 week old rat subependymal zone (Schmahl et al., 1989). Amazingly, this effect was still present 11 weeks after naltrexone treatment had ceased. Naltrexone, however, did not produce a difference in $\left[{ }^{3} \mathrm{H}\right]$ thymidine incorporation in the brain of rats younger than two weeks of age (Kornblum et al., 1987).

Seatriz and Hammer (1993) showed that naltrexone treatment from E12-P6 in the rat increased cortical thickness but did not have an effect on the number of neurons in the cerebral cortex. Zagon and McLaughlin (1986b) studied the effect of different doses of naltrexone on the postnatal cerebral cortex (P0-P21). This study showed that whereas high dose naltrexone (50 mg/kg/day, subcutaneous (s.c.) injection), or permanent opioid receptor blockade, decreased the density of neurons in the cerebral cortex (in accordance with the study of Seatriz and Hammer, (1993)), low dose naltrexone (1 mg/kg/day, s.c. injection), or temporary opioid receptor blockade, increased the density. These changes in density occurred simultaneously with changes in cortical thickness such that it was not likely that the absolute number of neurons in the cortex had changed.

In light of these data that support an inhibitory mode of action for opioid ligands and cellular proliferation in the developing rodent forebrain, it is surprising that acute morphine 
treatment increases $\left[{ }^{3} \mathrm{H}\right]$ thymidine uptake in the subependymal zone of the adult rat (Messing et al., 1979; Miller et al., 1982). Interestingly, in the same studies, naloxone by itself did not have an effect. This indicates that there is no tonic control by the opioid system over proliferation in the adult forebrain. Thus in the developing forebrain, there is a great paucity of data for how opiates affect proliferation during embryogenesis. However, the data that exists for opioids and cellular proliferation in the postnatal forebrain details a temporally sensitive relationship that requires further clarification.

\subsubsection{Opiates and Proliferation in the Cerebellum}

Cell counts demonstrated cell numbers in the cerebellum were markedly increased in both the internal granule layer and the molecular layer in response to naltrexone treatment (50 $\mathrm{mg} / \mathrm{kg} /$ day) in neonatal rats (P0 to P21) (Zagon and McLaughlin, 1983). Zagon and McLaughlin (1986a) added to their 1983 study by showing that as high dose naltrexone increased cerebellar size, low dose naltrexone reduced cerebellar size. During this postnatal period, proliferative activity in the cerebellum is also inhibited by beta-endorphin (Lorber et al., 1990).

Interestingly, in vitro, neural precursors taken from the EGL of the cerebellum significantly decreased proliferation in response to morphine (Opanashuk and Hauser, 1998; Hauser et al., 2000). Opanashuk and Hauser (1998) demonstrated that the substantial reduction in proliferation induced by morphine in EGL neuroblasts was completely overridden by 
heparin binding epidermal growth factor, suggesting a degree of interaction between opioid and EGF signaling pathways.

\subsubsection{Opiates and Proliferation in the Adult Hippocampus}

Unlike in the developing brain, where opioids fulfill an inhibitory function with regards to cellular proliferation, studies that have investigated the impact of opioidergic modulation of proliferation in the hippocampus have, at face value, been conflicting and are most likely reflective of an extremely complicated relationship between opioids and proliferation in the adult hippocampus. Chronic morphine treatment dramatically decreased cellular proliferation (Eisch et al., 2000; Mandyam et al., 2004) and decreased polysialylated NCAM expression (Kahn et al., 2005) in the adult rodent hippocampus. Importantly, morphine-induced decrease in hippocampal proliferation was also shown to be independent of hormone release from the adrenal gland, since rats that had undergone adrenalectomy showed the same antiproliferative response to morphine exposure (Eisch et al., 2000). Adrenal hormone release itself impacts upon neural proliferations (Gould et al., 1992). However, acute morphine exposure ( $2 \mathrm{~h}$ prior to BrdU labelling) did not inhibit proliferation (Eisch et al., 2000). Interestingly, in the same chronic morphine treatment regime in the mouse, morphine exposure elicited premature mitosis (Mandyam et al., 2004). These results appear contradictory to studies of morphine exposure in the hippocampus by Persson et al. (2003a; 2003b). 
In vitro, adult hippocampal progenitor cells (AHPs) were sensitive to both mu and delta opioid ligands. AHPs, however did not express KOR nor did they respond to its ligands (Persson et al., 2003a). MOR and DOR signalling in AHPs in vitro caused increased proliferation, indicating a direct signalling pathway for opioids and cellular proliferation in the hippocampus. Conversely, opioid antagonism decreases proliferation of AHPs in vitro (Persson et al., 2003b). Opioid antagonism with a non-selective opioid antagonist, naloxone, was found to decrease proliferation of AHPs. A similar result was seen with MOR selective antagonist, beta-FNA and DOR antagonist, naltrindole. Opioid antagonism with acute naltrexone also decreased cellular proliferation in the adult hippocampus (Holmes and Galea, 2002), although these results are directly contradictory to the studies by Eisch et al. (2000) and Mandyam et al. (2004) that showed an inhibitory effect of morphine, an agonist, on proliferation. Opioid receptor antagonism with naloxone (presumably through MOR and DOR) was also shown to affect differentiation (Persson et al., 2003b). After 10 days of treatment, naloxone decreased gliogenesis (for both astrocytic and oligodendrocytic markers). Naloxone exposure increased numbers of cells expressing the neuronal markers MAP2ab and alpha-internexin. These data suggest an anti-neuronal role for opioid signalling and differentiation in AHPs, which contrasts with the proneuronal differentiation observed in embryonic stem cells (Kim et al., 2006).

Proliferation in the hippocampus of spontaneously hypertensive rats is increased by chronic naltrexone treatment (Persson et al., 2004). However, this effect of opioid signalling on cellular proliferation is extremely context dependent. For example, in the same study it was shown that naltrexone decreased proliferation in the hippocampus after the rats had been 
exercising. Ra et al. (2002) demonstrated that exercise increases cellular proliferation in the adult hippocampus but failed to show an effect with naloxone. Persson et al. (2004) also demonstrated an increase in met-enkephalin in the hippocampus of exercising rats. This increase correlates well with the observed increase in proliferation (which, in the adult hippocampus, correlates mainly to neurogenesis (Harburg et al., 2007)).

Two recent studies using knockout (KO) mice have clearly shown that the endogenous opioid system modulates neurogenesis in the adult hippocampus. Kolodziej et al. (2008) using a MOR KO demonstrated that the KO mouse showed increased hippocampal neurogenesis after ischemic brain injury, while Koehl et al., (2008) using a beta-endorphin KO showed that the increased hippocampal proliferation due to exercise was dependent on beta-endorphin. These studies firmly establish a role for the opioid system in adult neurogenesis. This context dependency (exercising or sedentary) could explain the discordance between the studies of Eisch et al. (2000), Mandyam et al. (2004) and Kahn et al. (2005), with those of Holmes and Galea (2002) and Persson et al. (2003a; 2003b). Although beta-endorphin signals through both DOR and MOR, the selective DOR antagonist, naltrindole, had no effect on proliferation in the hippocampus of running rats (Persson et al., 2004) suggesting that MOR was the receptor involved in the observed modulation of hippocampal proliferation. 
As well as the classical opioid receptors (DOR, KOR and MOR), OGFr is proposed to mediate proliferation on stimulation with the opioid ligand, met-enkephalin (Zagon et al., 1999a). A large amount of material has been published on the effects of the opioid growth factor receptor by Zagon and colleagues detailing the inhibitory growth effects of the opioid growth factor receptor in cancer, angiogenesis and developing systems (reviewed in Zagon et al., 2002). However, this data awaits corroboration from other labs.

\subsubsection{Other Opioid Effects on Neuronal Cells in the Developing Brain}

As well as affecting neuronal proliferation, opiates also modulate other aspects of neurons such as spine formation and neurite outgrowth. Hauser et al. $(1987 ; 1989)$ demonstrated that chronic blockade of opioid receptors using naltrexone $(50 \mathrm{mg} / \mathrm{kg} / \mathrm{day})$ increased both dendrite length and spine number in the hippocampus, cerebral cortex and the cerebellum. Intermittent opioid receptor blockade using low doses of naltrexone $(1 \mathrm{mg} / \mathrm{kg} / \mathrm{day})$ inhibited growth of dendrites and spine number (Hauser et al., 1989). A later study showed that DOR stimulation also decreased neurite length, interpreted as evidence for a role in modulating differentiation (Hauser et al., 2000).

Methadone has been shown to reduce outgrowth from cerebellar explants taken from neonatal rat pups. Additionally, explants taken from the rat pups that were exposed to methadone during gestation also showed reduced outgrowth (Willson et al., 1976). In contrast, cellular and neuritic outgrowth from explants of embryonic and neonatal rat sympathetic and dorsal root ganglia and spinal cord were greatly enhanced by opioid 
peptide treatment (Ilyinsky et al., 1987). Thus opioid effects on outgrowth of neurites can be positive or negative depending on the region examined.

\subsubsection{Opiates and Gliogenesis}

A large body of literature that was established in the 1990s details how opiates impact upon macroglial proliferation, in vitro. Macroglia is the collective term for astrocytes and oligodendrocytes in the CNS. Astrocytes were once regarded as simply support cells for neurons. However, more and more research is outlining much more complicated roles for astrocytes that range from the expression of neuropeptide systems, such as the opioid system as well as assuming the role of neuronal progenitors (see below). Oligodendrocytes are involved with myelination in the CNS that is responsible for the efficient conductance of action potentials down axons.

\subsubsection{Expression of Opioid Receptors and Ligands in Macroglia}

Endogenous opioid signaling components have been characterised in macroglia of the developing brain. Opioid system ligands are expressed by astrocytes in primary culture (Vilijn et al., 1988; Hauser et al., 1990; Stiene-Martin et al., 1991a). Cultured astrocytes from neonatal rat brains expressed large amounts of proenkephalin mRNA and peptide that was regulated by cAMP signalling (Shinoda et al., 1989). Melner et al. (1990) found that only the flattened, adherent, type I astrocytes cultured from the neonatal rat brain, 
expressed proenkephalin mRNA, as opposed to the stellate type II astrocytes or the oligodendrocytes. Spruce et al. (1990) showed that proenkephalin protein was expressed by astrocytes in vitro when taken from P8 rats. This expression increases into adulthood. The same study also showed that immunohistochemical staining for proenkephalin peptide in histological sections of rat cerebellum first appeared at P5. Developmentally regulated temporal changes in proenkephalin mRNA expression also exist in the rat brain. In primary cultures of astrocytes taken from both the cerebellum and the cerebrum, proenkephalin mRNA expression increased from E20 to adulthood. Interestingly, met-enkephalin peptide was shown to decrease markedly from E20 to adulthood (Shinoda et al., 1992). In addition to temporal patterns of enkephalin expression, astrocytes also display differential spatial patterns. This was cited as an example of how astrocytes mimic what were once considered to be the exclusive properties of neurons (Shinoda et al., 1989; Ruzicka et al., 1995).

Macroglia also express opioid receptors. Astrocytes taken from 1 day-old mice expressed DOR, KOR and MOR (Ruzicka et al,. 1995; Stiene-Martin et al., 1998). Strikingly, opioid receptor expression was shown to interact with the cell cycle. Of the neonatal astrocytes that expressed MOR and DOR, a disproportionately large number of these astrocytes were in the $\mathrm{G}_{2} / \mathrm{M}$ phase of the cell cycle as compared to their non-opioid receptor expressing counterparts (Stiene-Martin et al., 1998). As well as differential temporal expression patterns, astrocytes also exhibited differential opioid receptor expression profiles depending on which part of the brain they were cultured from (Ruzicka et al,. 1995). Oligodendrocytes, in vitro, expressed both MOR and KOR, in that order during differentiation (Knapp et al., 1998). In vivo, opioid receptors on macroglia showed cell and 
developmental-specific expression patterns. A higher percentage of both GFAP-positive astrocytes and O4-positive oligodendroglia expressed opioid receptors in the SVZ of the P5 mouse than in the adult mouse SVZ. Of the opioid receptors, MOR was the most highly expressed (Stiene-Martin et al., 2001).

\subsubsection{Opioids and Macroglial Proliferation}

Morphine (Stiene-Martin et al., 1991b; Stiene-Martin and Hauser, 1993; Hauser et al., 1996), and other opioid receptor ligands (Stiene-Martin and Hauser, 1990; Hauser and Stiene-Martin, 1991; Stiene-Martin and Hauser, 1991) have been shown to consistently decrease DNA synthesis in cultured type I astrocytes taken from neonatal rodent brain. Just as proenkephalin expression in primary cultures of astrocytes and opioidergic modulation of proliferation in the postnatal forebrain displayed developmentally regulated patterns of activity, opioid-induced reductions in proliferation of macroglia, in vitro, also displayed temporal patterns (Hauser and Stiene-Martin, 1991). Cultured astrocytes taken from neonatal mouse brains showed reduced proliferation in response to met-enkephalin treatment, when taken from P3 or P5 mouse brains. Inhibition of proliferation was not seen in astrocytes taken from P19 mice. Intriguingly, astrocytes did not display decreased proliferation when taken from E19 mouse brains early during cell culture (within 6 days in vitro). However, cells were affected by met-enkephalin when maintained in culture for 11 days or more, suggesting that additional maturation was needed before the astrocytes became receptive to opioid mediated antiproliferative affects (Hauser and Stiene-Martin, 1991). Stiene-Martin et al. (2001) showed that morphine negatively, and naltrexone 
positively influenced DNA synthesis in astroglia within neonatal (P2-5) murine SVZ and striatum. In contrast to astrocytes, immature oligodendrocytes show increased proliferation in vitro with a MOR specific ligand, PL017. This effect was not observed in more mature oligodendroglia (Knapp et al., 1998).

\subsubsection{Opioid Receptor Signalling Pathways Associated With Proliferation}

Considerable work has gone into elucidating the relationship between opioids, proliferation and mitogen activated kinase (MAP kinase) pathway signalling. The MAP kinase pathway is important for cellular proliferation (reviewed in Raman et al., 2007) and neuronal differentiation of embryonic stem cells (Li et al., 2006). Opioid receptors have been shown to influence ERK phosphorylation in AHPs (Persson et al., 2003b), embryonic stem cells (Kim et al., 2006) as well as in glial cell lines (Bohn et al., 2000a,b; Belcheva et al., 2003; Belcheva et al., 2005). However, different opioid receptors stimulate the ERK pathway via different mechanisms (Belcheva et al., 2005). Belcheva et al. (2005) provided evidence which supports the following series of signalling events from MOR and KOR stimulation in astrocytes. Signalling via MOR with DAMGO in neonatal rat cortical astrocytes initiated a signalling cascade that activated phospholipase $\mathrm{C}$ (PLC) via calmodulin release from MOR (c.f. Wang et al., 1999), resulting in the generation of the secondary messenger, diacylglycerol (DAG). DAG release stimulated the phosphorylation of protein kinase C (PKC)-epsilon. PKC-epsilon is thought to activate the metalloprotease, A Disintegrin And Metalloproteinase (ADAM) which has been shown to release epidermal growth factor (EGF) ligands from the cell membrane (Prenzel et al., 1999). This stimulated EGF 
receptors (Belcheva et al., 2000; 2003) and ultimately, the MAP kinase pathway (Belcheva et al., 2005). In systems that do not express EGF, such as C6 glioma cells, MOR signalling transactivated the fibroblast growth factor (FGF) receptors (Belcheva et al., 2002).

KOR signals to ERK via a mechanism that differs from MOR-ERK signalling. KOR mediated release of the G-beta-gamma subunit activated phosphoinositide-3-kinase (PI3K)gamma. This was proposed to activate the atypical PKC-zeta via a PIP $_{3}-3$ '-PI dependent protein kinase 1 complex (c.f. Hirai and Chida, 2003). PKC-zeta then mobilized calcium ions. KOR trans-activation of the EGF pathway was dependent on calcium release, and it is thought that this may modulate proteases such as ADAM (Belcheva et al., 2005). The calcium dependent nature of KOR signalling and proliferation is corroborated by studies from the Hauser group. As well as inhibition of proliferation, morphine exposure also caused premature maturation in astrocytes which occurred through a calcium dependent mechanism. This effect was shown to be a result of KOR, rather than MOR stimulation by using receptor specific ligands (Stiene-Martin et al., 1993). Signaling through KOR was subsequently shown to increase intracellular calcium while decreasing proliferation (Gurwell et al., 1996).

Chronic morphine inhibits astrocyte replication via MOR signalling through a mechanism that depends on intracellular calcium stores and calcium mediated calcium release (Hauser et al., 1996). This effect was only seen after $48 \mathrm{~h}$ of exposure, thus, chronic opioid treatment at the cellular level has dramatically different effects from acute exposure (measured in intervals that last 5-10 min in studies by Belcheva et al. (2005) on cultured 
astrocytes). Interestingly, chronic ( $>2 \mathrm{~h}$ ) MOR and KOR activation induces a decrease in EGF induction of ERK phosphorylation in COS-7 cells (Belcheva et al., 1998). In support of a mechanism by which opioids signal via receptor tyrosine kinases, morphine was not able to induce any inhibitory effect on proliferating neuroblasts in the presence of heparinbinding-EGF (Opanashuk and Hauser, 1998).

These studies demonstrate that opioid receptors can signal to the MAP kinase pathway via transactivation of EGFR. Transactivation of EGFR via liberation of membrane EGFs such as heparin-binding-EGF by ADAM metalloproteases (Prenzel et al., 1999) has emerged as an important mediator of GPCR signaling (Rozengurt, 2007). A number of different GPCR agonists can signal via 'triple-membrane passing signaling' via EGFR including angiotensin II, ATP, bombesin, bradykinin, carbachol, endothelin, phenylephrine, thrombin (reviewed in Wetzker and Bohmer, 2003), as well as the cannabinoid receptor agonists, anandamide, THC and Win55,212-2 (Hart et al., 2004). Although signaling from GPCRs to the MAP kinase pathway may be modified by more classical means of intracellular signaling. This includes activation of PLC, generation of DAG, and phosphorylation of PKC, raf, MEK and ERK (Rozengurt, 2007). Activation of ERK itself has been implicated with proliferation in a number of ways and has been labeled as a regulator of $G_{1}$ to $S$ phase transition (Meloche and Pouyssegur, 2007). Roovers and Assoian (2000) suggested that both the duration as well as the intensity of ERK activation impacts on proliferation, as ERK activation has been associated with both proliferative and anti-proliferative effects. Whereas strong induction of ERK followed by sustained moderate activation leads to 
proliferation, a strong induction followed by sustained strong induction will arrest the cell cycle.

Opioidergic activation of ERK is context dependent, which is reminiscent of the extremely variable, or case dependent relationship between opioids and proliferation that is demonstrated in the literature. Opioidergic activation of ERK in embryonic stem cells is sustained over $24 \mathrm{~h}$ and is responsible for neuronal differentiation (Kim et al., 2006). In the same study, opioidergic stimulation of ERK in neuronal progenitor cells (which are analogous to radial glia and express astrocytic markers such as astrocyte-specific glutamate transporter (GLAST) (Bibel et al., 2004)) was shown to be transient and independent of observed antiproliferative effects (Kim et al., 2006). Rat cortical astrocytes display only transient increases in ERK phosphorylation upon DAMGO administration, whereas, U69,593, a KOR agonist, elicits a sustained activation. KOR signalling with U69,593 in C6 glioma cells is potently mitogenic (Bohn et al., 2000a). In the same astrocytic glia, MOR signalling strongly counters the proliferative effects of kappa opioid signalling as well as the mitogenic effect of endothelin by inhibiting phosphoinositide and MAP kinase signalling pathways (Bohn et al., 2000b). It is interesting that opioids have shown very different effects in vitro in what are, apart from embryonic stem cells, all essentially cells that display astrocytic markers. However, these studies illustrate at a cell autonomous level, that opioidergic signalling affects proliferation, potentially and interestingly through a second extracellular receptor tyrosine kinase signalling system, the EGF receptor. 
Opioid receptor signalling is also influenced by receptor desensitisation. MOR desensitisation is considered a mechanism that participates in opiate tolerance (Christie, 2008). MOR internalisation can contribute to receptor desensitisation. After activation by an agonist (such as DAMGO), MOR is phosphorylated by G-protein-coupled receptor kinase 2 which results in binding of beta-arrestin 2. This can lead to receptor internalisation by a dynamin dependent-dependent mechanism (Connor et al., 2004). Interestingly, morphine induced MOR desensitisation can occur in the absence of beta-arrestin 2 (Chu et al., 2008).

The literature published on how opioids affect development has several main themes. Opioids have a negative impact on growth in the developing brain, apart from in neuronal progenitor cells taken from the human, where KOR activation stimulates proliferation (Sheng et al., 2007). There is a large body of literature to show that opioids inhibit proliferation in macroglia, but again, this has its exceptions. In the adult, the relationship between opioids and proliferation is context dependent and appears unpredictable. The complicated relationship between cellular proliferation (particularly in the adult brain) and opioids mirrors the many levels of complexity resident within opioid system signalling. Opioid ligands display often promiscuous opioid receptor binding. A cell may express any one or any combination of the three opioid receptors, which are all under complicated transcriptional control. Of the three opioid receptor genes, multiple pharmacological classes and splice variants (at least for MOR) exist. Opioid receptors may also homo or heterodimerise. Each of these receptors may associate with different subtypes of G-protein and are capable of signalling through multiple secondary messengers, as well as different 
receptor systems. Context dependency of opioid signalling effects on development on both a cell type-specific as well as a temporal level has been noted previously (Hauser and Mangoura, 1998). Even given all of this, opioid receptor knockout animals demonstrate that opioids are not strictly necessary for normal development and therefore are only a part of a milieu of incoming antiproliferative/proliferative signals that interact and impact upon each other.

When all the data is taken into account, it supports the idea that the opioids represent a system that a developing or proliferating system may use to modulate or alter events that are already unfolding and are largely under the control of other molecular systems and programs. The idea that the opioid system occupies a lower-level role in development and proliferation that influences more dominant molecular systems operating at the time, accounts for the often contradictory and complicated observations that arise from research that involves opioids and proliferation in the CNS. Perhaps the answer to the question "what is the role of the opioid system in development and proliferation?" is simply, "it depends on what molecular systems are already operating within a proliferative environment". Further research on opioids and development must therefore be placed within the specific context of the whole developing brain. This prospect is exciting given the huge increase in knowledge on brain development that has been generated by means of sensitive genetic and molecular techniques (reviewed in Rakic, 2006) since the 1980s and 1990s when most of the background studies on brain development and opioids were conducted. 


\subsection{The Cerebral Cortex}

The cerebral cortex is arguably the one structure that makes us human (Rakic, 2006). The cerebral cortex is massively expanded in primates, and it is thought that the large size of the human cerebrum is due to mutations in genes that are attributed to microcephaly, a disease characterised by reduced cranial volume and mental retardation. These genes are all associated with the mitotic spindle and are thought to be involved with determining asymmetric or symmetric cellular divisions in the embryonic brain (reviewed in Cox et al., 2006). Interestingly, an allele of the ASPM gene, the most frequently mutated gene in microcephaly, is still under positive selection pressure in the human species, suggesting human brains are still evolving (Mekel-Bobrov et al., 2005).

Each hemisphere of the cerebrum in humans is divided into different lobes that are made of convoluted sulci and gyri to allow for efficient packaging of cortical tissue. These lobes are the frontal, parietal, temporal and occipital lobes. The frontal lobe contains the primary motor cortex that controls movement in the body in conjunction with the basal ganglia, cerebellum, brain stem and spinal cord. The parietal lobe contains the primary somatosensory cortex that contains a map of the sense of touch of the human body. The temporal cortex contains the auditory primary cortex, and the occipital lobe houses the primary visual cortex. The cerebral cortex is also responsible for aspects of our personality (particularly the frontal lobe), language, memory and integration of sensory information in regions of association cortex (adapted from Kandel, 2000). 


\subsubsection{Cerebral Development}

The human brain develops from the primary cerebral vesicles. These consist of the hindbrain (rhombencephalon), the midbrain (mesencephalon) and the forebrain (prosencephalon). The rostral portions of the prosencephalon that contribute to the formation of the cerebral cortex gives rise to the telencephalon. After the prosencephalon develops, two lateral diverticula appear that form the optic vesicles. Before the end of the fourth week of gestation, rudiments of the cerebral hemispheres appear as two "pouches" either side of the forebrain. The growing cerebral hemispheres of the telencephalon proceed to enlarge forwards, upwards and backwards such that the cerebral hemispheres acquire an oval shape. It is in this way that the medial regions of the cerebral hemispheres become separated by the longitudinal fissure. The very large cerebrum present in mammals, especially in humans, continues to grow caudally to overlap the diencephalon (by the second month of gestation), mesencephalon and the cerebellum. The surface of the cerebrum remains smooth until the beginning of the fourth month. By the seventh month all major sulci are present as the cerebral cortex expands in size (adapted from Warwick and Williams, 1973). How the cerebral cortex develops at the cellular level, however, is currently a subject of much research.

\subsubsection{Histogenesis of the Cerebral Cortex}

After splitting of the preplate to form the marginal zone and the subplate, the cerebral cortex forms in an inside-out manner (Angevine and Sidman, 1961) in a tight 7-day period 
of cellular proliferation during late gestation in the mouse (E12 to E18) (Takahashi et al., 1996a); corticogenesis takes almost 17 times longer in humans, but the process is similar (reviewed in Caviness Jr. et al., 1999). In the mouse (a highly utilised animal model for corticogenesis), the 7-day 'neurogenic interval' is comprised of 11 integer cell cycles (Takahashi et al., 1995a). Cellular proliferation that fuels generation of the cerebral cortex occurs in the VZ (so named by the Boulder Committee (1970)), a pseudo-stratified epithelia which rests below the developing cortical plate (Fig. 1.2, panel B). The VZ consists of two broadly defined populations of cells - the proliferative pool of cells $(\mathrm{P})$ and the quiescent, migrating neuroblasts that have exited the cell cycle (Q) (Takahashi et al., 1993). Once neurons have been born in the VZ, they migrate up towards the developing cortical plate via the radial glial scaffold. This idea was first put forward by Rakic (1972) and Levitt and Rakic (1980), who used histological analysis of glial fibre tracts and migrating neuroblasts to obtain clues to the cells' developmental relationships. In the rodent, excitatory neurons migrate radially up radial glial fibres from the VZ of the dorsal telencephalon. In contrast, inhibitory neurons that populate the murine cerebral cortex must migrate from the medial and lateral ganglionic eminences, in the ventral telencephalon, in a tangential manner (Fig. 1.2, panel A) (reviewed in Parnavelas, 2000; Nadarajah et al., 2003).

\subsubsection{A Model of Corticogenesis}

Corticogenesis in the mouse has been divided into three stages (Smart, 1973), based on the type of cell output of the VZ. In the developing mouse telencephalon, stage one (E10 to E12) is dominated by proliferation that enlarges the progenitor cell pool of the VZ, that is, 
no differentiation takes place. Stage two (E13) is characterised by a balance between proliferation and differentiation. Stage three (E14 to E18) is dominated by neurogenerative proliferation with the progenitor cell pool beginning a steady decline. By E18 the pseudostratified epithelium of the VZ is replaced by the cuboidal epithelium of subependymal layer (Takahashi et al., 1995a). Previously, it was thought that neuronal corticogenesis ceased at the end of gestation in the mouse. Astonishingly, however, GFAPpositive astroglia in the P5 mouse brain were shown to be the ancestors of neurons that integrated into the cerebral cortex (Ganat et al., 2006).

During corticogenesis in the mouse embryo, proliferative cells undergo a lengthening in cell cycle that had been described in a number of studies using cumulative BrdU labelling. At E11, proliferative cells had an average cell cycle time of $8.1 \mathrm{~h}$ (Takahashi et al., 1995a). At E16, the practical limit of cumulative BrdU labelling in the VZ, the cell cycle increased to $18.4 \mathrm{~h}$. This was due to a 4-fold increase in $\mathrm{G}_{1}$ phase (Takahashi et al., 1995a). Although the vast majority of work on the developing cerebral cortex has been conducted in the rodent, the primate neurogenic interval was characterised by an increase in cell cycle time from $22.7 \mathrm{~h}$ at $\mathrm{E} 40$ (the onset of primate neurogenesis in the cerebral cortex) until midcorticogenesis when the cell cycle time was $54.1 \mathrm{~h}$. Surprisingly, this was followed by a large decrease in cell cycle time to $27.4 \mathrm{~h}$ at the commencement of genesis of layer III of the cerebral cortex (Kornack and Rakic, 1998).

As cell cycle time increases in the rodent VZ, so does neuronal output. Two fractions of cells exist within the neurogenic embryonic VZ, the P and Q cellular fractions. At E11, the 
$\mathrm{P}$ fraction for the VZ is 1 (Takahashi et al., 1996a). This means that all divisions within the VZ will produce two more proliferative cells. As corticogenesis progresses, this $\mathrm{P}$ fraction steadily decreases. By E14, after 8 of the 11 neurogenic cell cycles have elapsed, the $\mathrm{P}$ fraction drops below 0.5 . This marks the point at which the VZ stops growing, and begins to shrink. This continues until E17, at which point it disappears entirely and is replaced by the ependymal layer that surrounds the LV in the adult. A simple relationship between Q (for a given cell cycle) and cell cycle number has been proposed (Caviness et al., 1999). Q is proportional to the square of the cell cycle number $(\mathrm{CC}$, which in the mouse, for example, can be anything from 1 to 11 ). This relationship is also modified by a constant, $\mathrm{k}$, which is species specific.

$\mathrm{Q}=\mathrm{kCC}^{1.97}$

The total neuronal output from the neurogenic interval is modified by the constant $\mathrm{k}$, which is 0.009 in the mouse. This produces 11 integer cell cycles in which the cerebral cortex is produced (at which time, $\mathrm{Q}=1$ ). By decreasing this species-specific constant, the total output of the neurogenic interval is increased. For example, to produce a brain the size of a monkey's, $\mathrm{k}$ drops to 0.0016 . This results in a neurogenic interval that is 28 cell cycles long.

The observation that cell cycle length increases with neuronal output is more than just correlation. An elegant study by Calegari and Huttner (2003) showed that neurogenic progenitors (Tis21-positive) had longer cell cycle times than their proliferative 
counterparts. Using cumulative BrdU labelling to give an indication of cell cycle time, it was reported that neuronal progenitors in the same neuroepithelia, at the same time in development, had different cell cycle times depending on what progeny they were committed to produce. These observations prompted a theory of neurogenesis, put forward by Götz and Huttner (2005). Essentially intrinsic determinants that influence the fate of the cell (to remain within the $\mathrm{P}$ fraction or to become a part of the $\mathrm{Q}$ fraction) exert their effect during a certain part of the cell cycle, i.e.: $\mathrm{G}_{1}$ phase. However, these determinants require time to work. Therefore, a cell that cycles through $\mathrm{G}_{1}$ quickly will not be sufficiently influenced to leave the cell cycle and will remain proliferative. However, a cell that takes a long time to transition through $\mathrm{G}_{1}$ will incur a higher probability of cell cycle exit.

The rate of change of Q for cells in the VZ does not vary and is proposed to be guided almost solely by cell-intrinsic mechanisms (Caviness Jr. et al., 1999). It has previously been shown that early neuronal progenitor cells which produce neurons destined for the lower (VI and V) cortical layers, can contribute to upper cortical layers when transplanted to a developmentally more mature VZ (McConnell, 1988). However, the reverse is not true. Progenitor cells taken from an older developing cortex cannot generate earlier, lower layers of cortex (Frantz and McConnell, 1996). These studies show cell intrinsic fates are important in neuronal maturation; however, cells can also be manipulated by the surrounding environment. The idea that developmental maturation of cortical progenitors is guided by cell intrinsic mechanisms gained further support with the discovery that before the first quiescent cell emerges in the telencephalon, some clonal lineages are already entirely restricted to glial fates (McCarthy et al., 2001). Shen et al. (2006) further 
elaborated on cell intrinsic cues within the developing cerebral cortex by showing that cortical progenitor cells taken from E10.5 mouse brains and grown in vitro first gave rise to Cajal-Retzius cells, followed by deep layer neurons and finally upper layer neurons. CajalRetzius cells are a transient population of cells that inhabit layer I of the cerebral cortex and express the large extracellular protein, reelin. They are thought to play important roles during development of the cortex which range from modulation of neuronal migration to radial glial maturation (Soriano and Del Rio, 2005). The study by Shen et al. (2006) demonstrated that before E10.5, a progenitor cell is programmed to produce a complex order of neuronal progeny.

Experimental validation of the model of corticogenesis put forward by Takahashi and colleagues has involved genetic manipulation of the cell cycle regulatory gene, p2 $7^{(\mathrm{Kip} 1)}$ (p27), which is expressed at high levels during mid corticogenesis (Delalle et al., 1999). p27 is a CDK inhibitor that checks the cell cycle at the $\mathrm{G}_{1}$ restriction point and is responsible for lengthening of $\mathrm{G}_{1}$ phase (Mitsuhashi et al., 2001). Cells that pass this point in the cell cycle will pass through to mitosis. Cells that leave the cell cycle must leave at this restriction point. p27 activity increases the likelihood that a cell will exit the cell cycle and join the Q fraction. The E14 p27 KO mouse was shown to already have a cerebral wall 23\% larger than its wild-type littermates (Caviness et al., 2003) since a higher proportion of proliferative divisions expands the progenitor cell pool. The leaving $\mathrm{Q}$ fraction at this stage was 5\% lower than normal littermates. At P21, the p27 KO mouse possessed $23 \%$ more supragranular cerebral cortex (layers III and II) (Goto et al., 2004). There was no change in cell density but interestingly there was a $17 \%$ increase in excitatory projection neurons. 
GABAergic inhibitory neurons were not overrepresented in layers II and III of the p27 KO mouse cortex. Conversely, over expression of p27 during cycles 3-4 of the neurogenic interval decreased the thickness of the cerebral cortex at P21 (Tarui et al., 2005). Contrary to expectation, in the same study, p27 over-expression in the E14 mouse VZ did not increase the duration of $\mathrm{G}_{1}$, nor did it increase the neurogenic interval. Collectively, these studies demonstrate that generation of the cortical layers can be manipulated via cellintrinsic control over the cell cycle.

\subsubsection{Radial Glia - Neuronal Progenitors}

The radial glia that populate the VZ were recently discovered to be the neuronal progenitor cells of the CNS (Malatesta et al., 2000; Noctor et al., 2001; Miyata et al., 2001; Malatesta et al., 2003; Anthony et al., 2004), although the clonal relationship between radial glia and neurons was previously suggested by Gray and Sanes (1992) who used retroviral labelling of radial glia in the embryonic chicken optic tectum. Golgi staining revealed that once neurogenesis was over, radial glial cells became the astrocytes of the adult brain (Schmechel and Rakic, 1979). 


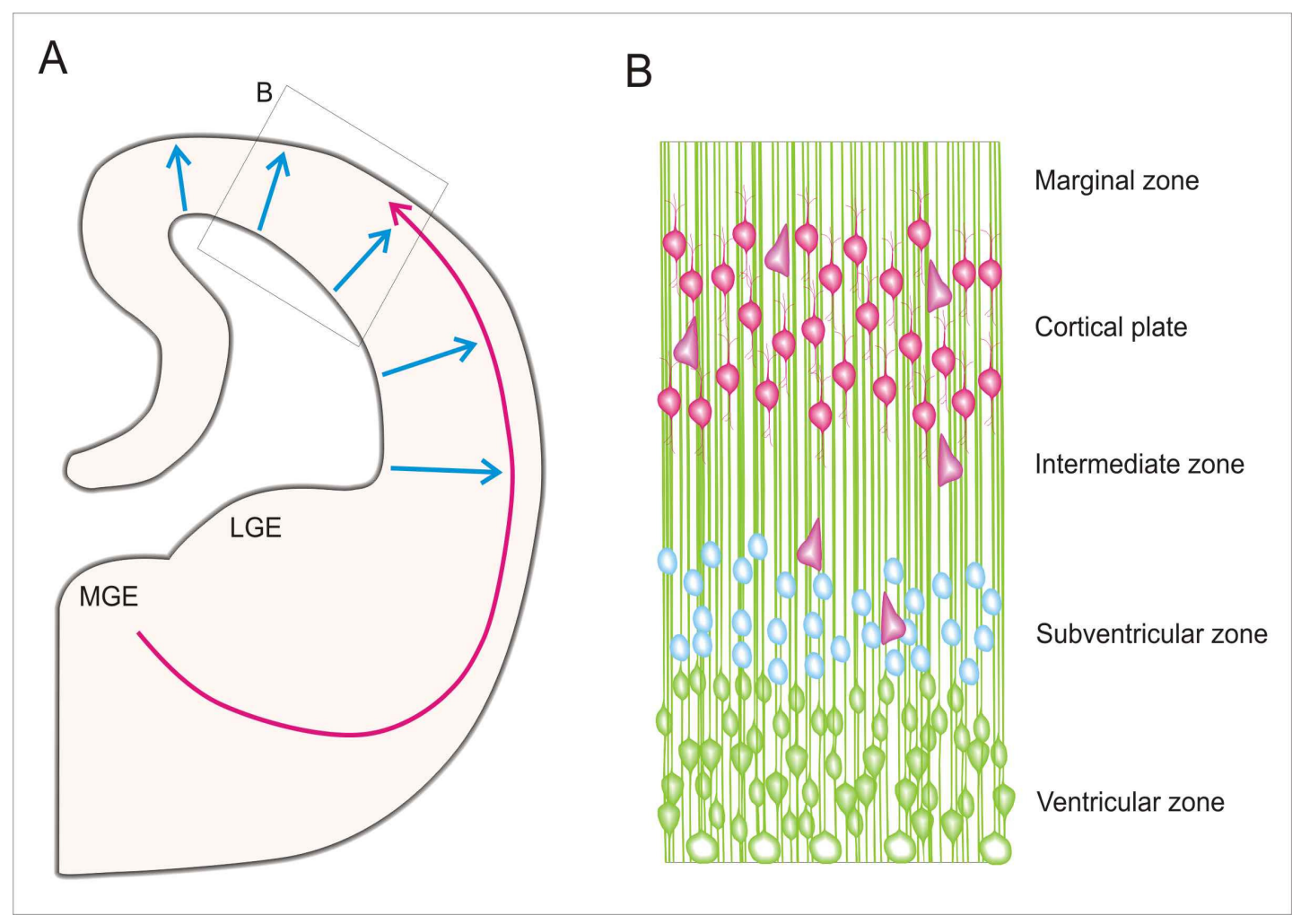

Fig. 1.2. Neuronal migration and nomenclature in the developing cerebral cortex. Panel A shows types of neuronal migration in the embryonic rodent cortex. Excitatory neurons that arise within the dorsal telencephalon migrate radially outward towards the cortical plate (blue arrows). Inhibitory GABAergic neurons are born in the ventral rodent telencephalon, within the medial and lateral ganglionic eminences (MGE and LGE, respectively). These neurons must migrate tangentially into the cortical plate (red arrow) (adapted from Parnavelas, 2000). Panel B shows a transverse section of developing cortex (enlarged from panel A). The VZ, a pseudo-stratified epithelium which sits on top of the $\mathrm{LV}$, is populated by radial glia (green), which give rise to neurons that migrate into the cortical plate via the radial glial scaffold.

Neurogenesis also occurs in the SVZ from basal progenitor cells (blue). The cell sparse intermediate zone separates the SVZ from the developing cortical plate (red cells) (panel B is adapted from The Boulder Committee (1970)).

At the onset of neurogenesis, neuroepithelial cells that form the lining of the neural tube differentiate to form radial glia. Both radial glia and neuroepithelial cells undergo a process 
called interkinetic nuclear migration (INM) (see text below); however, unlike radial glia, neuroepithelial cell nuclei migrate along the entire cell length that extends from the ventricular surface to the outer pial surface; whereas, radial glial cell nuclei are restricted to the VZ. Both types of cell express nestin (reviewed in Pinto and Götz, 2007) and RC2 (Hartfuss et al., 2001) and both have apical-basal polarity. Importantly, at a molecular level, differentiation to radial glia involves expression of astrocytic markers such as brainlipid binding protein (BLBP), GLAST, vimentin and in the primates/human, glial fibrillary acidic protein (GFAP) (reviewed in Götz and Huttner, 2005). Hartfuss et al. (2001) proposed that radial glia represented a heterogeneous population, based on astrocytic antigen expression profiles. However, Anthony et al. (2004) have proposed that radial glia represent a homogenous population, but that radial glia at different stages of development express different antigens. Anthony et al. (2004) showed that all radial glia expressed both GLAST and BLBP at some stage in their development. The first study to find direct evidence that radial glia are neuronal progenitors was Malatesta et al. (2000), after finding that mouse embryonic brain cells that were FACS sorted for the glial antigen, GLAST, could generate neurons when seeded onto a developing rat cortex.

Radial glial contributions to the final neuronal population of the CNS is a topic of debate. Malatesta et al. (2003) used Cre-recombinase under control of a human GFAP promoter that induced the expression of a reporter construct to show that the vast majority of neurons in the cerebral cortex were derived from radial glia. Interestingly, these authors also showed that relatively few neurons in the basal ganglia originate from radial glia ( $21 \%$ in the striatum and $13 \%$ in the globus pallidus). Furthermore, they showed that although most 
glutamatergic projection neurons had radial glial ancestry, most GABAergic interneurons did not. In stark contrast to this study by Malatesta et al. (2003), Anthony et al. (2004) argued that nearly all neurons of the brain, even those of the ventral telencephalon, showed evidence of radial glial ancestry. Like Malatesta et al. (2003), Anthony et al. (2004) used a Cre-recombinase reporter fate mapping system. However, Anthony et al. (2004) used a BLBP promoter to drive Cre-recombinase expression. Whereas the human GFAP promoter is only expressed in the ventral telencephalon from E14.5, BLBP is expressed several days earlier. This makes a big difference between the two fate mapping experiments since by E14.5, the ventral telencephalon is largely gliogenic (Anthony et al. 2004).

\subsubsection{Radial Glial Mitotic Outcomes}

During neurogenesis, radial glial mitoses can have a number of outcomes. Neurons that are born at the ventricular surface in the E14 mouse inherit the basal radial glial process to give rise to a "radial neuron" that undergoes somal translocation to reach the cortical plate (Fig. 1.3 E) (Miyata et al., 2001). Miyata et al. (2004) detailed the behaviour of radial glia around the time the cortical plate emerges in the mouse (E13 to E14). They found that at this stage, most radial glial cell divisions at the ventricular edge produced two proliferative daughter cells $(\mathrm{P} / \mathrm{P}$ divisions, $\mathrm{P}=$ proliferative $)$. Several different types of $\mathrm{P} / \mathrm{P}$ divisions can be achieved at the ventricular wall. After a radial glial cell undergoes a P/P division at the ventricular surface, the proliferative daughter cells may both migrate back to the ventricular surface to divide. This is called a type I division. However, after the initial surface division, one of the proliferative daughter cells may remain near the SVZ (becoming an intermediate 
progenitor cell) to divide and produce two quiescent neurons $(\mathrm{Q} / \mathrm{Q}$ divisions, $\mathrm{Q}=$ quiescent). This is referred to as a type II division.

Interestingly, in the stage two VZ, type II divisions preferentially occurred beneath thicker (thus more developmentally advanced), lateral regions of cortical plate. Type II divisions can be further broken down into two groups (Fig. 1.3 B and C). Type IIa divisions occur when the intermediate progenitor cell inherits the basal (or pial) process from its mother cell. In type IIb divisions, the non-surface dividing cell inherits the ventricular process. In both cases, the vast majority of intermediate progenitor cell divisions have been shown to produce two quiescent neurons, identified by expression of Hu RNA binding protein, a neuronal cell marker.

To add further complexity to progenitor cell types in the VZ of the developing rodent, Gal et al. (2006) showed that radial glial cells share the VZ with another type of progenitor cell, the short neuronal precursor. In contrast to the radial glial cell that spans the entire developing cortex with its basal process, the short neuronal precursor cell has an apical process and a basal process of variable length (Gal et al., 2006). Thus whereas modern studies of radial glia have proposed that radial glia are solely responsible for neurogenesis out of the VZ, the actual situation in the embryonic rodent VZ may more closely match that of the human, where neuronally restricted precursor cells that are not radial glia have been characterised (Zecevic, 2004). In saying this, it cannot be ruled out that short neuronal precursor cells are derived from radial glia. 


\subsubsection{Radial Glia and INM}

During cellular division, progenitor cell nuclei in the VZ undergo the process of INM, as first described by Sauer (1935), whereby the nuclei migrate between the basal VZ, where they reside during S phase, and the apical surface of the VZ, where mitosis occurs. This process occurs in neuroepithelia throughout the developing CNS, including the developing retina. Here, INM is thought to heavily influence neurogenesis by positioning cell nuclei in apical-basal neurogenic gradients (Baye and Link, 2007a). The nucleus accomplishes this migration by using the motor protein kinesin to pull itself along the microtubules in its ascent to the basal regions during $\mathrm{G}_{1}$ phase and dynein and dynactin during its descent in $\mathrm{G}_{2}$ phase to the apical VZ (reviewed in Baye and Link, 2007b).

Migration of proliferative nuclei within the VZ is not a smooth movement. Takahashi et al. (1993) revealed that INM to the apical surface of the VZ was a process in which movement varied as the nucleus accelerated and stalled. This was confirmed by real-time imaging of INM (Tsai et al., 2005). At E14, it was observed that nuclei descending to the apical surface descended in two different phases. The first half of the descent was completed in $\mathrm{S}$ phase, which took approximately $4 \mathrm{~h}$. The second half of the descent which occurs during $\mathrm{G}_{2}$ phase was observed to take only $1 \mathrm{~h}$ (Takahashi et al., 1993). 


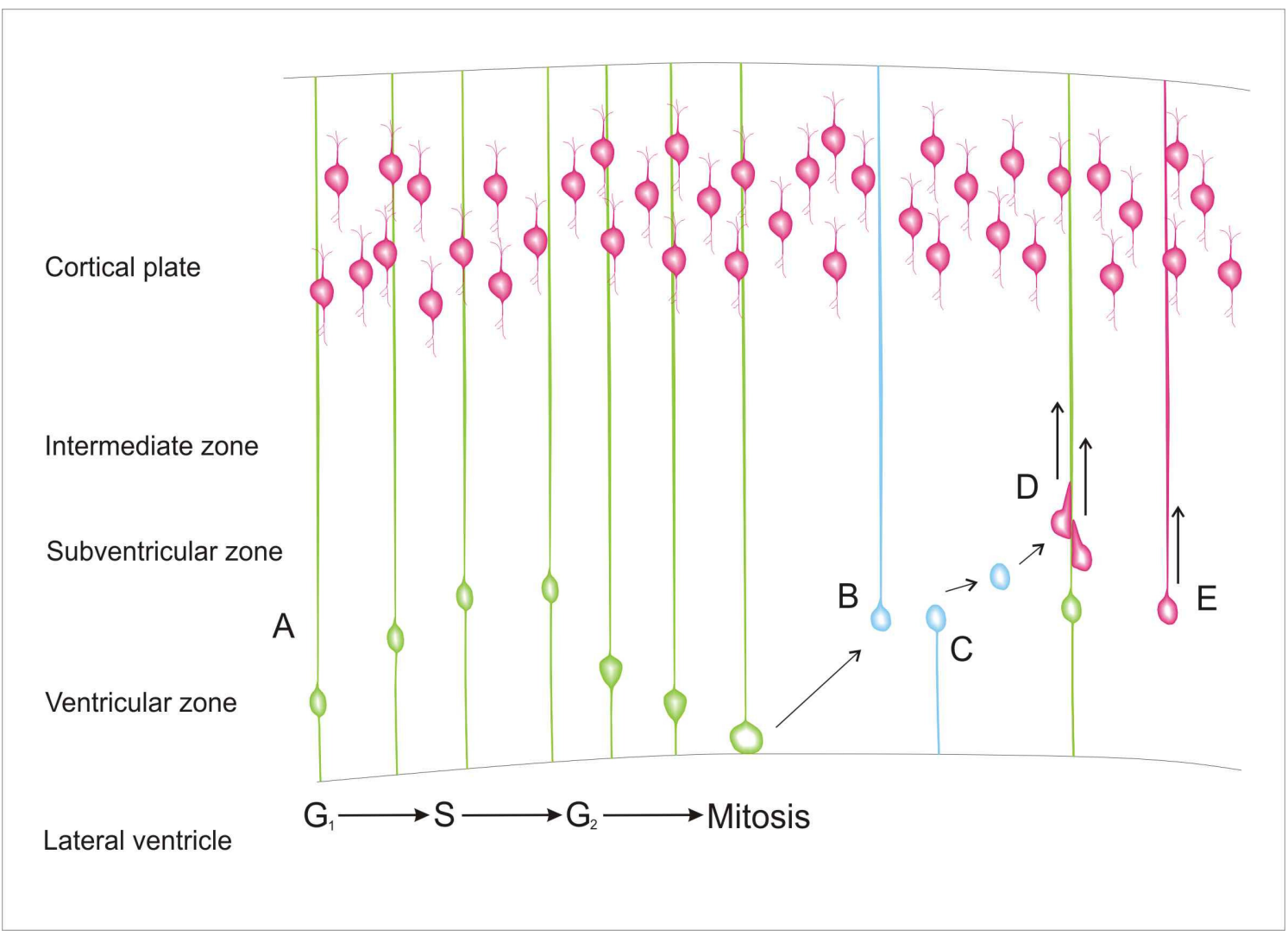

Fig. 1.3. INM and neurogenic divisions within the dorsal telencephalon. A) Radial glia (green) display nuclear migration within the VZ. During $\mathrm{G}_{1}$ phase of the cell cycle, radial glial nuclei migrate away from the LV to undergo $S$ phase. At the start of $\mathrm{G}_{2}$ phase, the nucleus rapidly descends to the ventricular surface to undergo mitosis. If the radial glial cell produces a basal progenitor cell, the basal progenitor (blue) may inherit either the basal (B) or the apical (C) process. After loss of these processes, the basal progenitor cell will produce two quiescent neurons that will migrate to the cortical plate (red cells) (D). Alternatively, a radial glial cell may produce a neuron at the ventricular, or apical surface of the VZ. The neuron inherits the basal process of the radial glial cell and migrates by somal translocation to the cortical plate (E).

The functional consequences of INM in the CNS are poorly understood; however, it seems that the relationship between cell cycle and INM can be easily uncoupled. Previously, perturbation of INM in the Cdc42 KO mouse (Cappello et al., 2006) as well as in the Pax6 KO rat (Tamai et al., 2007) resulted in an uncoupling of $\mathrm{G}_{2}$ phase time and INM. In both 
studies, INM perturbation caused ectopic mitoses. Murciano et al. (2002) and Messier and Auclair (1974) demonstrated that pharmacological inhibition of INM with cytochalasin B, which inhibits actin polymerisation, in other neuroepithelia also led to ectopic mitoses, since $\mathrm{G}_{2}$ phase was left unchanged.

As it has been documented that inhibition of INM does not impact upon $\mathrm{G}_{2}$ phase time, it has also been shown that interrupting the cell cycle at $\mathrm{S}$ phase does not disturb INM (Murciano et al., 2002). Another study, however, showed that knocking out the amyloid precursor protein gene produced a slowing in $\mathrm{G}_{2}$ phase (Lopez-Sanchez et al., 2005) without ectopic mitoses. Ueno et al. (2006) showed that cell cycle progression is required for normal INM to the apical surface of the VZ; however, treatment with the powerful cell cycle inhibitors 5-azacytidine and cyclophosphamide induced massive apoptosis within the VZ.

In an attempt to identify functional consequences of INM, Murciano et al. (2002) investigated the relationship between INM and the anti-neurogenic gradient of Notch1Delta1 (Notch1 ligand) expression and signalling. Interestingly, expression of Notch1 was restricted to the ventricular wall (where radial glial nuclei are positioned during late $\mathrm{G}_{2} / \mathrm{M} /$ early $\mathrm{G}_{1}$ phases), and BrdU labelling showed that Notch1 was expressed by cells in $\mathrm{G}_{2}$ phase, but not by cells in $\mathrm{S}$ phase or early $\mathrm{G}_{2}$ (Murciano et al., 2002). It was shown that INM positions cells such that notch1 expressing neuronal progenitors are exposed to the maximum amount of anti-neurogenic ligand, Delta1, which is also expressed by cells at the apical VZ. Pharmacological perturbation of this positioning resulted in less anti-neurogenic 
Notch1 signalling and more neurogenesis, which, in turn, would decrease the final cumulative neuronal output.

\subsubsection{Role of the SVZ in Foetal Corticogenesis}

Neurogenesis also occurs in the foetal SVZ, where basal neuronal progenitors undergo mitosis (Haubensak et al., 2004; Miyata et al., 2004; Calegari et al., 2005). The proliferative cells that make up the SVZ are a secondary proliferative population and were originally thought to be largely gliogenic, owing to their contribution to the glial cell pool in the postnatal brain (reviewed in Noctor et al., 2007). Study of cell cycle and proliferative behaviour in this zone was slow due to the fact that there is no hard boundary between the $\mathrm{VZ}$ and the SVZ, as well as the fact that the population of the SVZ is intermingled with postmitotic cells (Takahashi et al., 1995b). These basal progenitor cells do not undergo INM (Smart, 1973), and most undergo terminal divisions to generate two neurons during embryogenesis (Noctor et al., 2004). Interestingly, Tarabykin et al. (2001), using the noncoding RNA, Svet1, as a SVZ marker, provided evidence to support the idea that the SVZ may be largely responsible for generation of the upper layers of the cerebral cortex. This hypothesis was supported by the fact the SVZ undergoes massive expansion during generation of the upper cortical layers (Noctor et al., 2007). However, unlike the SVZ of the developing rodent, which primarily produces excitatory neurons, the dorsal SVZ of the human is also a major source of GABAergic interneurons for the cerebral cortex (Letinic et al., 2002). In the rodent, most GABAergic interneurons are derived from the ventral telencephalon. 


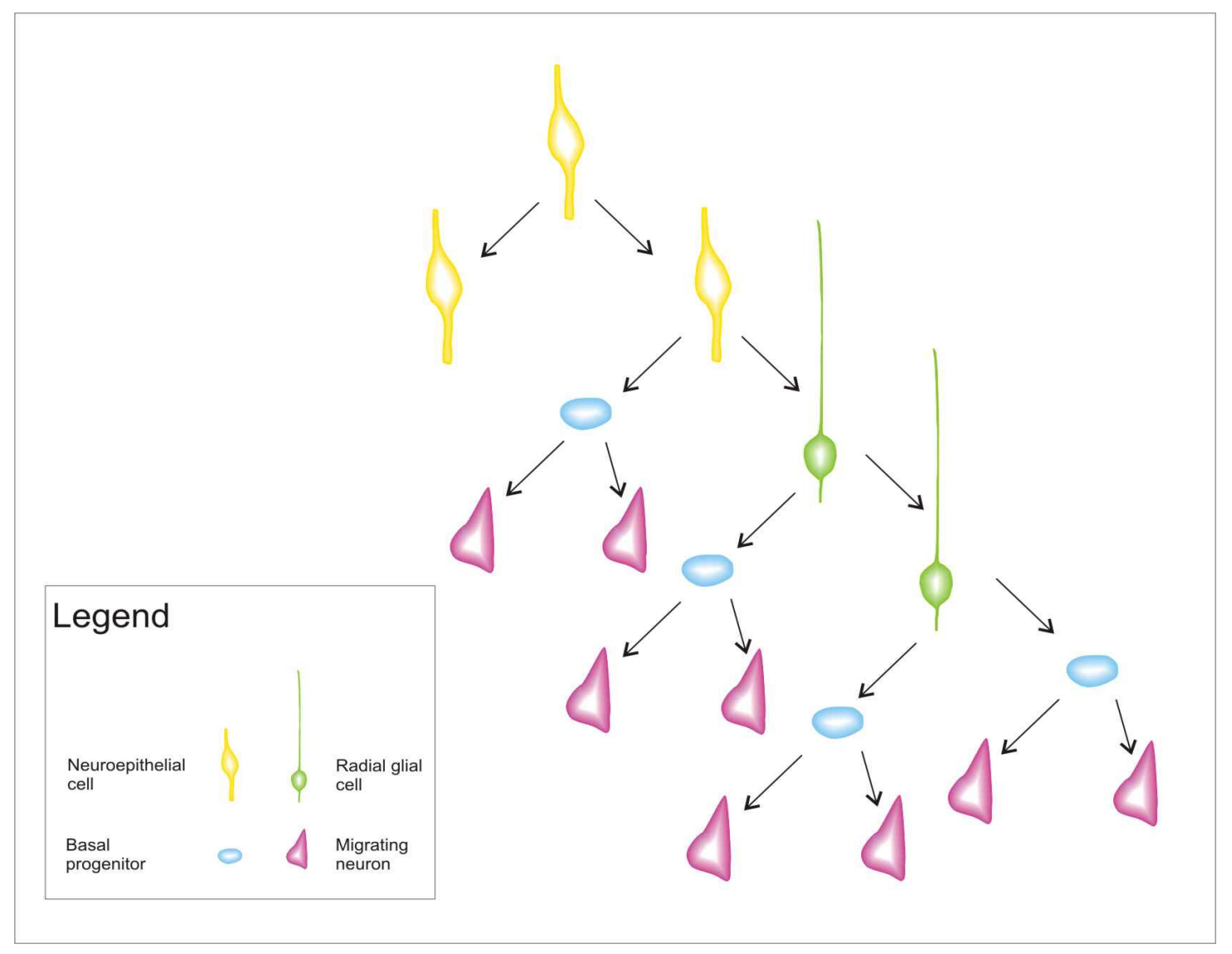

Fig. 1.4. A simplified diagram of neuronal lineage. Neuroepithelial cells, which exist in the VZ prior to neurogenesis, give rise to radial glial cells. Radial glial cells can produce neurons via basal progenitor cells (adapted from Götz and Huttner, 2005). Note that this diagram does not describe astrogliogenesis.

\subsubsection{Postnatal Neurogenesis in the Mammalian Forebrain}

In the neonatal mouse, radial glial cells are present until at least P7, where they still proliferate and even undergo INM (Tramontin et al., 2003). However, unlike the VZ that eventually becomes replaced by a single cell ependymal layer, the SVZ survives past corticogenesis where neurogenesis and gliogenesis manages to persist into adulthood (Lois 
and Alvarez-Buylla, 1993). Neuronal proliferation around the lateral ventricles fuels the rostral migratory stream with migratory neuroblasts destined to differentiate into different types of interneurons within the olfactory bulbs (Young et al., 2007). Interestingly, the rostral migratory stream has recently been identified within the human telencephalon (Curtis et al., 2007a), however, not without controversy (Sanai et al., 2007; Curtis et al., 2007b).

The existence of neuronal stem cells around the adult lateral ventricles in the rat and mouse is a relatively recent finding. Doetsch et al. (1999) were the first to show that these adult neural stem cells were phenotypically astrocytes. Neuronal production around the LV originates at the astrocytic neuronal stem cells (type B cells of the adult SVZ) and proceeds through to a progenitor cell step (type C cells, or transit amplifying cells) that produces the migrating neurons of the rostral migratory stream (type A cells) (Doetsch et al. 1999). It seems as if these astrocytic neuronal stem cells represent the last vestiges of their neurogenic radial glial ancestors, in mammals at least. Merkle et al. (2004) used Cre-loxP based fate mapping to show that type B cells of the SVZ were descendants of radial glia that existed in the P0 mouse forebrain. Thus, even though rodent radial glia have all but disappeared from the VZ by the second week of life, neurogenic potential in the postnatal brain continues in the form of astrocytes.

Our understanding of development of the cerebral cortex has increased dramatically in recent years. It is now apparent that although extrinsic signalling does play some part in corticogenesis, developmental progression within the embryonic brain seems to be 
controlled by cell-intrinsic mechanisms, which includes cell cycle progression. Importantly, the past decade has seen the rise of the astrocytic glial cell as the major source of neurons for the entire CNS. Remarkably, astrocytes continue to proliferate in the adult brain and provide a source of neurons. It is therefore interesting that postnatal astrocytes have been shown to express a fully functional opioid signalling system and proliferating astrocytes taken from the postnatal brain are very sensitive to the antiproliferative affects of opiates. Unfortunately, by the time it was determined that radial glia produce neurons for the embryonic brain, and that astrocytes are responsible for adult neurogenesis, studies on how opiates impact on astrocytic proliferation had largely ground to a halt, particularly in vivo, perhaps for want of a reason to continue. With this in mind, the current research project aimed to characterise the impact of opiate exposure in the context of the embryonic brain. This general aim consisted of 3 objectives. Firstly, expression of MOR in the prenatal brain was analysed on a single-cell level. The second objective of this thesis research was to characterise the effects of morphine exposure on the proliferating VZ. The final objective was to analyse the effect of morphine exposure during corticogenesis on histogenesis of the cortical plate. 


\section{General Methods}

\subsection{Animals}

All experiments involving animals were approved by the Victoria University Animal Ethics Committee.

\subsubsection{Timed Mating}

C57BL/6J mice were mated overnight (9 pm - $9 \mathrm{am})$ at the Malaghan Institute of Medical Research. All mice had access to food and water ad libitum and were separated during pregnancy. Basic environmental enrichment was provided. Mice that had vaginal plugs present in the morning were considered E0. Mice were taken for experiments on E15 or E18 or were left until parturition and taken for tissue dissection at P5 or P12. At the end of the experimental protocol, pregnant dams were sacrificed by cervical dislocation. An incision was made from the pelvis to the ribcage and uterine horns were removed and put into ice-cold PBS. Foetuses were dissected out of the uterus and were blotted briefly, then weighed to gauge developmental progress of the foetus before further dissection. Postnatal pups were sacrificed by decapitation. 


\subsubsection{5-Bromo-2'-Deoxyuridine Injections}

Animals that received a single injection of 5-bromo-2'-deoxyuridine (BrdU, Sigma-Aldrich, $858811-500 \mathrm{MG}$ ) received it as an intraperitoneal injection of PBS containing $10 \mathrm{mg} / \mathrm{ml}$ of BrdU at a dose of $50 \mathrm{mg} / \mathrm{kg}$. Animals were sacrificed at different times after injection of BrdU.

\subsection{Molecular Analysis}

\subsubsection{Real-Time PCR Analysis of DCX Gene Expression}

\subsubsection{Tissue Dissection}

Cerebral cortical tissue was dissected from E18.5 mouse cerebral cortex and was immediately homogenized in $100 \mu \mathrm{l}$ of PBS in $1.5 \mathrm{ml}$ microcentrifuge tubes, using a small plastic pestle. An aliquot of cortical homogenate $(20 \mu \mathrm{l})$ was transferred to $200 \mu \mathrm{l}$ of TRIzol (Invitrogen, 15596-018). Both the PBS homogenate and the TRIzol homogenate were immediately frozen at $-80{ }^{\circ} \mathrm{C}$ until further use.

\subsubsection{RNA Extraction}


Upon thawing, TRIzol samples were centrifuged at $13,000 \mathrm{x}$ g for 15 minutes at $4{ }^{\circ} \mathrm{C}$ to remove lipid and cellular debris. Supernatant was transferred to a new microcentrifuge tube. Chloroform $(100 \mu \mathrm{l})$ was added to every $1 \mathrm{ml}$ of TRIzol supernatant, and the mixture was mixed by vortexing, then centrifuged at $13,000 \mathrm{x} g$ for $10 \mathrm{~min}$ to separate the organic phase from the aqueous phase. The aqueous phase was collected by micropipette and mixed with 0.5 volumes of absolute ethanol. The sample was then further purified using a High Pure RNA Isolation Kit (Roche, 1828 665). The resulting RNA was eluted in $40 \mu$ of $\mathrm{ddH}_{2} \mathrm{O}$. RNA was quantified with an ND-1000 Spectrophotometer (Nanodrop, Wilmington, DE) by obtaining an absorbance at $260 \mathrm{~nm}$ and a $260 \mathrm{~nm} / 280 \mathrm{~nm}$ ratio of absorbance to give an indication of contamination of the RNA by protein as well as phenol from the RNA extraction process.

\subsubsection{Reverse Transcription}

RNA (300 ng) was reverse transcribed into cDNA by Superscript III (Invitrogen, 18080085). Reverse transcription reactions included RNAseOUT (Invitrogen, 10777-019) to inhibit RNAse A, B and C activity. Oligo $\mathrm{dT}_{18}(2.5 \mu \mathrm{M})$ was used as a primer to selectively reverse transcribe messenger RNA.

\subsubsection{Real-Time PCR}


One $\mu$ l of cDNA (Superscipt III reaction mixture) was used per $25 \mu$ l PCR reaction. PCR reactions were based on Platinum SYBR Green qPCR SuperMix-UDG (supplied at 2x concentration, Invitrogen, 11733-046) to which template (cDNA), primers (final concentration of $200 \mathrm{nM}$ ) and fluorescein were added. Fluorescein was added to the reaction mixture at a final concentration of $20 \mathrm{nM}$. Fluorescein was necessary for the iQ4 real-time PCR detection system based on an iCycler platform (Bio-rad, Hercules, CA) to collect background well-factors. Well factors are collected from individual wells at the beginning of a PCR experiment and are used to correct for non-uniformity in fluorescence (usually as a result of pipetting error).

Cycling conditions for the real-time PCR reaction were as follows. An initial step of $50{ }^{\circ} \mathrm{C}$ for 2 min was included before the PCR amplification to eliminate contaminating PCR product. This was achieved using uracil-N-glycosylase, which removes uracil residues from DNA molecules. As Platinum SYBR Green qPCR SuperMix-UDG (Invitrogen, 11733-046) contains dUTP instead of dTTP, contaminating PCR product from prior PCR amplification is digested by uracil-N-glycosylase while cDNA template, which contains dTTP, remains intact. Denaturation was carried out at $95{ }^{\circ} \mathrm{C}$ for $2 \mathrm{~min}$. Amplification was carried out over 35 cycles of $95{ }^{\circ} \mathrm{C}, 15 \mathrm{~s} ; 60{ }^{\circ} \mathrm{C}, 30 \mathrm{~s} ; 72{ }^{\circ} \mathrm{C}, 30 \mathrm{~s}$. Sixty ${ }^{\circ} \mathrm{C}$ was used as the primer annealing temperature for all primer sets used (Table 2.1).

\subsubsection{Agarose Gel Electrophoresis}


PCR product was visualised using agarose gel electrophoresis. Agarose gels (2\%) were prepared by microwaving $1 \mathrm{~g}$ of agarose (Invitrogen, 15510-027) with $50 \mathrm{ml}$ of $1 \mathrm{x}$ TAE buffer. Aqueous ethidium bromide $(10 \mathrm{mg} / \mathrm{ml})$ was added to the molten agarose to a final concentration of $200 \mathrm{ng} / \mathrm{ml}$. Agarose gels were placed into an electrophoresis tank with $1 \mathrm{x}$ TAE buffer containing $200 \mathrm{ng} / \mathrm{ml}$ ethidium bromide. Ten $\mu \mathrm{l}$ of neat PCR product was mixed with $2 \mu$ of $6 x$ loading buffer by pipetting up and down. PCR product mixed with loading buffer $(10 \mu \mathrm{l})$ was loaded into each lane of the agarose gel. Electrophoresis was conducted at $100 \mathrm{~V}$ for $25 \mathrm{~min}$. Ethidium bromide fluorescence was visualised using a KODAK Gel Logic 100 system (Kodak).

\begin{tabular}{lcc}
\hline \multicolumn{1}{c}{ Primer target } & \multicolumn{2}{c}{ Primer sequence 5’ $-3^{\prime}$} \\
\cline { 2 - 3 } & \multicolumn{2}{c}{ Forward } \\
\hline MOR & CCT GCC GCT CTT CTC TGG TT & CGC ATG GGT CGG ACT GGT TG \\
DCX & CAT AGT GCC CAC TGT AGC TTC & CAG ACA AGG ATC GGG TCA \\
Fas & AGC TGA GGA GGC GGG TTC AT & CAT TCT TCC ATA TCG GGC AC \\
Cyclophilin A & GGG GAG AAA GGA TTT GGC TA & ACA TGC TTG CCA TCC AGC C \\
Glyceraldehyde-P & GTG CCA GGC GTG TCA T & GAA GCG TTC GTG AAT AAC CTT \\
dehydrogenase & & \\
(GAPDH) & & \\
18s rRNA & CTT TGG TCG CTC GCT CCT C & CTG ACC GGG TTG GTT TTG AT \\
\hline
\end{tabular}

Table 2.1: Primers used for real-time PCR analysis.

\subsubsection{Western Blotting}




\subsubsection{Protein Extraction}

E18.5 cerebral cortical extract in PBS was solubilised in RIPA buffer. A mammalian protease inhibitor cocktail (Sigma, P8340-5ML) was added to slow protease-mediated protein degradation. Protein extracts were sonicated to break up viscous gDNA using an Ultrasonic Processor sonicator (Heat Systems Ultrasonics, Inc., Farmingdale, NY) at the settings: cycle time, $5 \mathrm{~s} ; \%$ duty cycle, $20 \%$; output control, 2.5 .

\subsubsection{Protein Quantification}

Protein quantification was achieved using a bicinchoninic acid (BCA) based assay (Pearce, 23225). This assay uses BCA to chelate cuprous ions $\left(\mathrm{Cu}^{1+}\right)$ after they have been reduced from cupric ions $\left(\mathrm{Cu}^{2+}\right)$ by protein in an alkaline environment. Chelation of cuprous ions by two molecules of BCA produces a purple product that absorbs strongly at $562 \mathrm{~nm}$. Absorbance at $562 \mathrm{~nm}$ has a linear relationship with increasing protein concentration.

\subsubsection{Protein Electrophoresis and Membrane Transfer}

Protein extracts were diluted to a concentration of $1 \mu \mathrm{g} / \mu \mathrm{l}$ in lithium dodecyl sulfate (LDS) sample buffer (Invitrogen, NP0007). Protein samples were reduced with reducing agent (Invitrogen, NP0004) at $70{ }^{\circ} \mathrm{C}$ for $10 \mathrm{~min}$, then $20 \mu \mathrm{g}$ of protein was loaded per lane of a 412\% ZOOM gel (Invitrogen, NP0923BOX), and the samples were electrophoresed at $100 \mathrm{~V}$ 
for approximately $1 \mathrm{~h}$ in MES buffer (Invitrogen, NP0002). The electrophoresed protein was run with Seeblue II protein size standard (Invitrogen, LC5925). Protein was transferred to Immobilon-FL PVDF membrane (Millipore, IPFL00010), that had been pre-soaked in methanol, in transfer buffer at $20 \mathrm{~V}$ overnight. Only one brain sample was loaded per lane. Where littermates were used for western blotting, brain samples were electrophoresed, western blotted and quantified by densitometry separately, then averaged within the litter to obtain separate $n$ values.

\subsubsection{Antibody Incubation}

The next morning, the PVDF transfer membrane was rinsed in PBS then blocked in PBS containing $5 \%$ reduced fat trim milk for $2 \mathrm{~h}$ at room temperature. The PVDF membrane was then rinsed in PBS and incubated with PBS containing 2\% BSA and primary antibody for $4 \mathrm{~h}$. The membrane was washed in PBS containing 0.1\% TWEEN for $3 \times 5 \mathrm{~min}$, then incubated with secondary antibody conjugated with Alexa-488 (1:5,000, Invitrogen) for a further $2 \mathrm{~h}$. Membranes were washed as for the primary antibody and transferred protein bound to antibody visualised with a Fujifilm FLA5000 fluorescence scanner (Fujifilm Medical Systems, USA) at $600 \mathrm{~V}$.

\begin{tabular}{llll}
\hline Epitope & Animal primary & Company & Dilution \\
& antibody raised in & \\
\hline MOR & Rabbit & Chemicon, Temecula, & $1: 1,000$ \\
& & CA (AB5511)
\end{tabular}




$\begin{array}{llll}\text { DCX } & \text { Guinea Pig } & \text { Chemicon (AB5910) } & 1: 20,000 \\ \text { NeuN } & \text { Mouse } & \text { Chemicon (MAB377) } & 1: 1,000 \\ \text { GAD 65+67 } & \text { Rabbit } & \text { Abcam (AB11070) } & 1: 1,000 \\ \text { GFAP } & \text { Mouse } & \text { Chemicon (MAB360) } & 1: 1,600\end{array}$

Table 2.2: Primary antibodies used for western blotting.

\subsection{Immunohistochemistry}

\subsubsection{Tissue Preparation}

After removal from the uterus, foetuses at E15.5 were rapidly decapitated and whole heads were fixed overnight in PBS containing 4\% paraformaldehyde (PFA). The brains of E18.5 foetuses and P5 pups were dissected out in ice-cold PBS. Brains were fixed overnight in PBS containing 4\% PFA at pH 7.4. The next morning, brains destined for cryostat microtome sectioning were cryo-protected in PBS containing 30\% sucrose and stored at -80 ${ }^{\circ} \mathrm{C}$ until sectioning. Brains destined for paraffin embedding were dehydrated using a graded ethanol/xylene series and embedded in paraffin wax.

\subsubsection{Tissue Sectioning}

Fixed-frozen tissue was mounted in Jung Tissue Freezing Medium (Leica Instruments, Nussloch, Germany) and sectioned at $20 \mu \mathrm{m}$ (rodent tissue) or $40 \mu \mathrm{m}$ (human tissue) using 
an HM 500 OM series cryostat microtome (Microm, Heidelberg, Germany). Sections were mounted on Superfrost Plus microscope slides (Esco, Erie Scientific, Portsmouth, NH), air dried and stored at $-80{ }^{\circ} \mathrm{C}$ until staining.

Brains embedded in paraffin wax were sectioned on an 820 series microtome (American Optical Co., Buffalo, NY), and sections were mounted on Superfrost Plus microscope slides (Esco, Erie Scientific, Portsmouth, NH).

\subsubsection{Fluorescence Immunohistochemistry for Fixed-Frozen Brain Sections}

Frozen sections were air-dried and permeabilised at room temperature in PBS containing $0.05 \%$ Triton $\mathrm{X}-100$ for $15 \mathrm{~min}$ and blocked for $1 \mathrm{~h}$ at room temperature in PBS containing $2 \%$ BSA and $10 \%$ goat serum. Sections were incubated overnight at $4{ }^{\circ} \mathrm{C}$ with primary antibodies.

All antibodies were diluted in PBS containing 2\% BSA and 10\% goat serum. Sections were washed 4 times for 10 min each in PBS containing 0.1\% TWEEN. Sections were then incubated with the appropriate secondary antibodies for $1 \mathrm{~h}$ at room temperature. Sections were washed as for the primary antibody and mounted in Prolong Gold antifade reagent with DAPI for fluorescent visualisation of the cell nuclei (Invitrogen, P-36931). In the present study, DAPI was used as a fluorescent counterstain to help clarify anatomical topography where appropriate. 


\subsubsection{BrdU Staining for Fixed-Frozen Sections}

Frozen sections were thawed at room temperature and air dried. Sections were permeabilised at room temperature in PBS containing 0.05\% Triton X-100 for 15 min, then sections were washed in PBS for $2 \mathrm{~min}$, followed by a 5 min incubation in $40 \mathrm{mM}$ Tris (pH 8) containing $10 \mathrm{mM} \mathrm{CaCl}_{2}, 6 \mathrm{mM} \mathrm{MgCl}$ and $10 \mathrm{mM} \mathrm{NaCl}$ (DNAse I incubation buffer). DNAse I incubation buffer was then replaced with $150 \mu \mathrm{l}$ of DNAse I incubation buffer containing $250 \mu \mathrm{g} / \mathrm{ml}$ DNAse I (bovine pancreas, grade II, Boehringer Mannheim, 104 159). Sections were incubated with DNAse I for $10 \mathrm{~min}$ at $37^{\circ} \mathrm{C}$. Sections were washed $3 \mathrm{x}$ 2 min in PBS containing $10 \mathrm{mM}$ EDTA that chelated divalent cations and deactivated the DNAse I. Sections were then blocked in PBS containing 2\% BSA at room temperature for $1 \mathrm{~h}$. After incubation overnight at $4{ }^{\circ} \mathrm{C}$ with rabbit anti-MOR antibody, sections were washed $3 \times 5 \mathrm{~min}$ in PBS containing 0.05\% TWEEN. Sections were then incubated for $2 \mathrm{~h}$ with goat anti-rabbit-Alexa 555 antibody at room temperature and washed $2 \times 5$ min as above. Sections were then incubated with anti-BrdU Alexa-546 conjugate for $2 \mathrm{~h}$ at room temperature. Sections were washed once in PBS for $5 \mathrm{~min}$ and were immediately fixed in 4\% PFA for 5 min to prevent diffusion of the antibody (Appendix 3, panel C). Sections were washed again in PBS for 5 min and were mounted in Prolong Gold antifade reagent with DAPI. 


\subsubsection{Fluorescence Immunohistochemistry for Paraffin-Embedded Sections}

Paraffin-embedded sections were de-paraffinised in xylene and rehydrated through a graded ethanol series. Antigens were retrieved in a boiling water bath at $100{ }^{\circ} \mathrm{C}$ in $10 \mathrm{mM}$ sodium citrate, $\mathrm{pH} 6.0$ for $10 \mathrm{~min}$. The $\mathrm{pH}$ of this solution is of critical importance. Tissue sections were then left to cool in citrate solution for $30 \mathrm{~min}$.

For retrieval of the Ki67 epitope, sections were put straight into a pressure cooker filled with 21 of boiling $10 \mathrm{mM}$ sodium citrate, $\mathrm{pH}$ 6.0. The pressure cooker lid was then locked down and the sections were left for 10 min after the cooker had come up to pressure. After 10 min at pressure, the pressure cooker was de-pressurised, and the lid was taken off. Cold tap water was run into the pressure cooker for $10 \mathrm{~min}$. Ki67 required retrieval in a pressure cooker, which allows the sodium citrate to reach temperatures greater than $100{ }^{\circ} \mathrm{C}$. Unlike the other antigens, $\mathrm{Ki} 67$ could not be retrieved by boiling with citrate at $100{ }^{\circ} \mathrm{C}$.

All sections were processed as for the frozen sections with specific primary antibodies as described above. Bound primary antibodies were visualised by staining with appropriate fluorescent secondary antibodies. BrdU immunohistochemistry was also visualised by diaminobenzidine (DAB) staining using a Vectastain Elite ABC Kit (Vector Laboratories, Burlingame, CA) according to the manufacturer's instructions. Slides were mounted in either DPX (BDH, Poole, UK) or Prolong Gold antifade reagent as appropriate. 


\subsubsection{DAB Staining}

DAB (Sigma-Aldrich, D12384-1G) was used to stain BrdU-labelled nuclei via anti-BrdU (PRB-1)-biotin ABC staining. Immediately prior to staining, a $0.5 \mathrm{mg} / \mathrm{ml}$ solution of DAB was prepared in PBS. This yielded a clear solution with a white precipitate. Hydrogen peroxide was added to the solution to a final concentration of $0.03 \%$. DAB solution was incubated with tissue at room temperature until sufficient staining was evident. This usually took 10 min. DAB stained slides were counterstained using $0.2 \%$ thionin. Slides were dehydrated in non-reagent grade ethanol, cleared in xylene and mounted in DPX.

\begin{tabular}{|c|c|c|c|}
\hline Epitope & Animal & Source & Dilution \\
\hline MOR & Rabbit & Chemicon (AB5511) & $1: 1,000$ \\
\hline DOR & Rabbit & Chemicon (AB5503) & $1: 1,000$ \\
\hline KOR & Rabbit & Abcam (AB10283) & $1: 1,000$ \\
\hline GLAST & Guinea Pig & Chemicon (AB1782) & $1: 1,000$ \\
\hline Nestin & Mouse & $\begin{array}{l}\text { R\&D Systems } \\
\text { (Minneapolis, MN) } \\
\text { (MAB1259) }\end{array}$ & $1: 100$ \\
\hline Vimentin & Mouse & $\begin{array}{l}\text { DakoCytomation } \\
\text { (Denmark) (M0275) }\end{array}$ & $1: 100$ \\
\hline Doublecortin (DCX) & Guinea Pig & Chemicon (AB5910) & $1: 500$ \\
\hline PCNA & Mouse & Chemicon (CBL407) & $1: 50$ \\
\hline BrdU/IdU (PRB-1) - Biotin & Mouse & Invitrogen (A21301MP) & $1: 50$ \\
\hline BrdU/IdU (PRB-1) - Alexa 546 & Mouse & Invitrogen (A21308) & $1: 100$ \\
\hline BrdU/IdU (PRB-1) - Alexa 488 & Mouse & Invitrogen (A21303) & $1: 100$ \\
\hline BrdU (Br-3) - Biotin & Mouse & Invitrogen (MD5215) & $1: 25$ \\
\hline Neuronal nuclear antigen (NeuN) & Mouse & Chemicon (MAB377) & $1: 200$ \\
\hline
\end{tabular}


Table 2.3: Primary antibodies used for immunohistochemistry.

\begin{tabular}{lllll}
\hline Epitope & Conjugate & Animal & Source & Dilution \\
\hline Rabbit IgG & Alexa 555 & Goat & Invitrogen(A-21428) & $1: 500$ \\
Rabbit IgG & Alexa 488 & Goat & Invitrogen(A-11008) & $1: 500$ \\
Mouse IgG & Alexa 488 & Goat & Invitrogen(A-11001) & $1: 500$ \\
Mouse IgG & Alexa 555 & Goat & Invitrogen(A-21424) & $1: 500$ \\
Guinea Pig IgG & Alexa 488 & Goat & Invitrogen(A-11073) & $1: 500$ \\
Vectalab ABC & Streptavidin-HRP & NA & Vector Laboratories, & NA (Kit \\
Elite & & & Burlingame, CA (PK- & specifications) \\
& & & $6200)$ & \\
Biotin & & & Invitrogen(S-32354) & $1: 500$ \\
\hline
\end{tabular}

Table 2.4: Secondary antibodies used for immunohistochemistry.

\subsection{Statistical Considerations and Analysis}

\subsubsection{The Use of Multiparous Species in Developmental Studies}

Developmental studies that utilise multiparous species as model research systems, such as the mouse, need special consideration for statistical analysis. A litter effect often arises because littermates are more similar to each other than to animals of other litters (Holson 
and Pearce, 1992). This brings with it statistical complications that have been the subject of debate for decades, primarily in the field of teratology (Palmer, 1974; Weil, 1974).

In highly inbred animal models such as rodents, these shared similarities are likely to arise simply as a consequence of the shared maternal environment. This has been observed in parameters as diverse as morphological features, levels of neuroendocrine hormones, as well as teratological parameters (Haseman and Hogan, 1975; Holson and Pearce, 1992; Zorrilla, 1997). The consequence of designating littermates as single observations (or single ' $n$ 's) is to inflate the $\mathrm{n}$ value of the experiment in question. An inflated $\mathrm{n}$ value leaves the experiment open to a type 1 error, or rejection of the null hypothesis when the null hypothesis is in fact true. The seminal study by Holson and Pearce (1992) used MonteCarlo simulations to show that selection of even 2 pups per litter can lead to 3 fold inflation in alpha level.

The simplest and most robust way to circumvent confounding litter effects is simply to define a pregnant dam as the smallest sampling unit of an experiment. All results presented in this thesis use the pregnant dam as the base definition of $\mathrm{n}$. This minimises spurious findings from biased experiments that have not considered litter effects. However, this increases the cost and work involved in the study, since more pregnant dams are needed. Surprisingly, the risky practise of using littermates as a sampling unit is prolific in the literature (Haseman and Hogan, 1975; Holson and Pearce, 1992; Zorrilla, 1996; Wainwright, 1998). 


\subsubsection{Statistical Analysis of Variance}

All statistical analysis in the current thesis research was performed using SPSS for windows v14.0 (SPSS, Chicago, IL). Where only two means were compared, Student's ttest was used to determine equality of means. For analysis of variance where only one measurement was taken from an animal, the univariate procedure was used. For cases where repeated measurements were taken from a single animal, the GLM repeated measures procedure was performed. Repeated measures ANOVA also requires that sphericity is not violated. Mauchly's test of sphericity was used to test this assumption. Corrections for violations of sphericity are provided by SPSS in the form of GreenhouseGeisser, the Huynh-Feldt and the Lower-bound corrections. For analysis of variance tests, normality was determined by Q-Q plot and if necessary, transformed by taking the natural logarithm or the square root of the raw data. 


\section{MOR is Expressed on Radial Glia but not on Migrating Neurons in the Late Foetal Mouse Brain}

\subsection{Introduction}

In vitro studies suggest that opioids exert an inhibitory effect on cellular proliferation. Opioids also have a measurable effect in vivo on proliferating systems within the central nervous system. The presence of MOR within germinal zones of the developing foetal brain was anticipated but needed to be unequivocally established, since numerous studies have generated conflicting results with regard to the location of MOR mRNA, protein, and ligand binding within proliferative regions (Kent et al., 1982; Leslie and Loughlin, 1993; Georges et al., 1998; Leslie et al., 1998; Zhu et al., 1998; Reznikov et al., 1999; Tong et al., 2000).

Early studies in our lab by Drs. Mrkusich, Day and Miller noted the presence of MOR mRNA expression in the E16 rat VZ, using mRNA fluorescence in situ hybridisation (Mrkusich et al., 2004) (Fig. 3.1). It was important at the start to determine the expression patterns of MOR in the developing brain. Fluorescence immunohistochemistry was used to co-label expression with a selection of neuronal and glial markers that would reveal cells in which the receptor was expressed. Reznikov et al. (1999) found opioid receptor expression within the $\mathrm{VZ}$ of the developing mouse foetus but failed to distinguish exactly which cells 
the receptors were expressed on, noting they could be either migrating neurons or progenitor cells. It was decided to look for MOR expression at a time-point just before parturition in the mouse (E18.5) as well as a point during neurogenesis (E15.5). This decision was based on the studies of Kent et al. (1982), who showed opioid receptor radioligand binding in the ependymal zone of the rat just before parturition, and Rius et al. (1991) and Leslie et al. (1998) who demonstrated that the opioid peptides beta-endorphin and enkephalin, respectively, displayed peaks of expression just before birth,

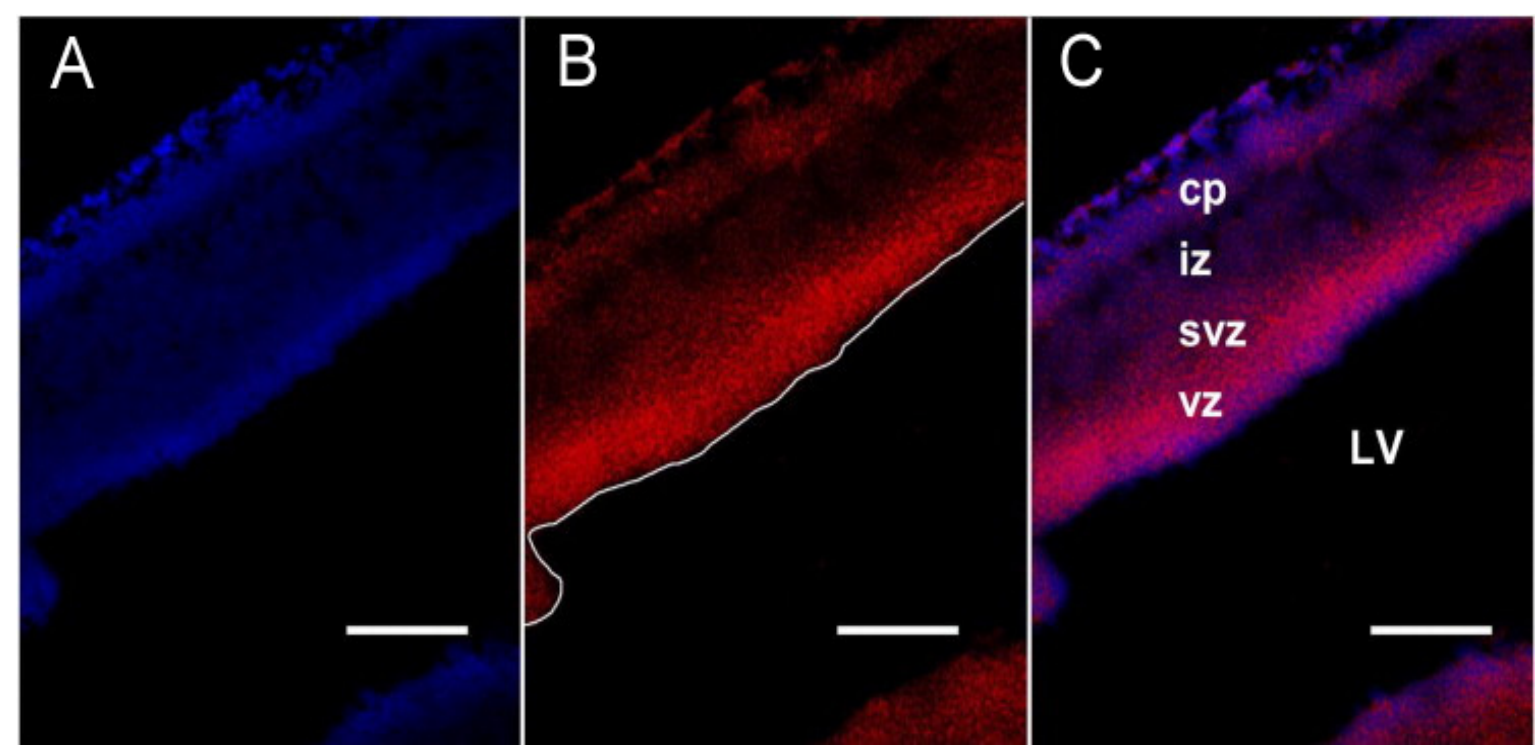

Fig. 3.1. MOR mRNA expression in the VZ of the E16 rat telencephalon. Cy3 labelled cRNA was used as a probe for fluorescence in situ hybridisation as described in Mrkusich et al. (2004) and Day et al. (2007). Panel A shows DAPI counterstaining of sagittal sections $(12 \mu \mathrm{m})$ through the developing cortex and associated dorsal proliferative regions. Panel B shows MOR mRNA hybridisation fluorescence signal, especially strong in the VZ. Panel C shows an overlay of panels A and B. Staining and photography was performed by Dr E.M. Mrkusich. Brain regions are labelled according to the Boulder Committee conventions (1970). cp, cortical plate; iz, intermediate zone; svz, subventricular zone; vz, ventricular zone. Scale bars: $200 \mu \mathrm{m}$ 
Localisation of MOR in the E18.5 mouse was determined in the neuroepithelia of the LV, the third ventricle $(3 \mathrm{~V})$, and the aqueduct $(\mathrm{Aq})$. As development of the cerebral cortex was the primary focus of the current study, analysis of the neurogenic VZ of the E15.5 dorsal telencephalon for expression of MOR, as well as DOR, was also conducted. Unfortunately an anti-KOR antibody that yielded convincing, specific staining on rodent tissue could not be found.

The proliferative epithelia of the E18.5 mouse still exhibit high levels of proliferation; however, it is gliogenesis, not neurogenesis, that predominates at this late stage of cellular proliferation in the late foetal mouse brain (Malatesta et al., 2000). Even though neurogenesis has largely ceased by E18.5, radial glial cells are still the dominant cell phenotype found in the lateral ventricle. GLAST, vimentin, and BLBP-positive radial glial cells are present in all regions of the late foetal mouse brain, including the telencephalon, diencephalon, midbrain, hindbrain/cerebellum, and the spinal cord (reviewed in Götz and Huttner, 2005). For localisation of MOR, GLAST was chosen as a radial glial marker. Data showing the subcellular localisation of GLAST on the radial glial cell has yet to be presented. However, glutamate transport by GLAST is vital for normal brain development thus it must, at least in part, be localised to the plasma membrane of the radial glial cell (Matsugami et al., 2006).

To complement GLAST, DCX was chosen to serve as a marker for migrating neuroblast cells. As MOR affects proliferation in the developing brain, PCNA (proliferating cell 
nuclear antigen) and BrdU were used as markers to assess whether or not MOR expressing cells were proliferative.

As a secondary proliferative zone dedicated to the production of neurons destined to reside within the internal granule layer of the cerebellum (reviewed in Goldowitz and Hamre, 1998), the EGL yields another opportunity to examine the relationship between MOR expression and regions of proliferative activity. Interestingly, MOR expression was shown on neuroblasts cultured from the EGL of the cerebellum (Hauser et al., 2000). As the cerebellum is a structure that develops extremely late in the brain, the cerebellum was probed for MOR expression at E18.5, P5, and P12.

\subsection{Experimental Design}

\subsubsection{Tissue Preparation and Staining}

Animals were taken for tissue preparation and sectioning at E15.5, E18.5, P5, and P12. Animals at E15.5 and E18.5 were injected with single doses of BrdU and sacrificed $3 \mathrm{~h}$ later for tissue processing. All staining was performed alongside negative controls in which the primary antibody had been omitted. 


\subsubsection{Image Acquisition and Analysis}

Analysis of different immunohistochemical markers was consistently performed on the same part of the brain by using anatomical landmarks such as the foetal striatum and the lateral-dorsal corner of the LV. Epifluorescent photomicrographs were taken with an Olympus AX70 photomicroscope (Olympus Optical Co., Hamburg, Germany) equipped with appropriate narrowband fluorescence filters. Images from the photomicroscope were captured using an Olympus DP70 CCD camera.

Confocal microscopy was used to determine co-expression or sub-cellular localisation of MOR with other markers in a single cell of a tissue section. Z-plane image stacks were acquired at $0.6 \mu \mathrm{m}$ intervals as this is the limit of resolution for confocal microscopy in the z-axis. Confocal microscopy was performed using either an MRC 600 Bio-rad confocal system (Bio-rad, Hercules, CA) with a Nikon Diaphot inverted microscope, a Leica TCS 4D confocal microscope (Leica Lasertechnik, Heidelberg, Germany), or an Olympus FV1000 (Olympus Optical Co., Hamburg, Germany). All three dimensional reconstructions, .avi files and three channel fluorescent images were constructed from Zstacks performed with the Olympus FV1000 confocal microscope. Photomicroscope and confocal images were overlaid and background corrected for presentation purposes using ImageJ v1.34s (NIH, USA) and Adobe Photoshop CS2 or CS3 software.

\subsection{Results}




\subsubsection{MOR Antibody Validation}

The anti-MOR antibody used in this study was raised against an exon 4 peptide in the carboxy-terminus of MOR. Correct specificity of the MOR antibody was demonstrated by obtaining the expected staining pattern in sections of the dorsal horn in mouse spinal cord (Fig. 3.2, panel A) and also by observing strong, diffuse staining of foetal (E18.5) striatum (Fig 3.2, panel B; Fig. 3.3, panels A and C). In contrast, patchy MOR staining was found in the P6 mouse striatum (Fig. 3.2, panel D). This was expected, given the ontogeny of MOR expression in the developing striatum. It has previously been shown that as the striatum develops, MOR adopts a patchy expression pattern (Winzer-Serhan et al., 2003) as demonstrated in Fig. 3.2, panel D. Additionally, lysates prepared from cortical dissections corresponding to the same cortical/ventricular regions displayed in Fig. 3.3 were examined by Western blotting and stained using the anti-MOR antibody (Fig. 3.2, panel C). A band at approximately $45 \mathrm{kDa}$, corresponding to the expected size of the unprocessed MOR protein, and a band at $70 \mathrm{kDa}$, corresponding to the glycosylated form of the receptor, were observed. 


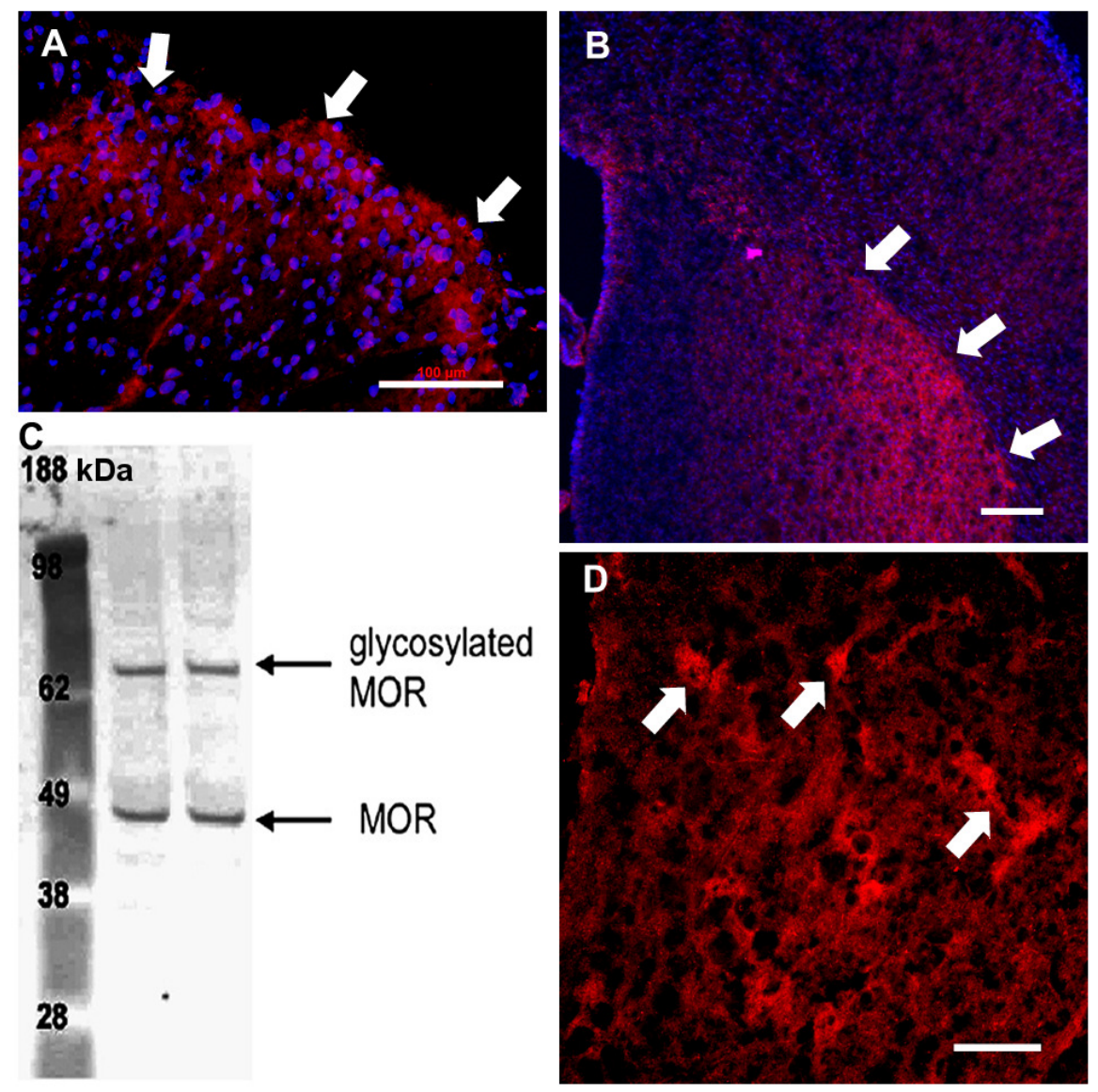

Fig. 3.2. Analysis of rabbit anti-MOR specificity. Panel A shows MOR staining (red) in a transverse section of the adult spinal cord. MOR was expressed strongly in the dorsal horn (white arrows) where MOR regulates sensory neurons. Nuclei were counterstained with DAPI (blue). Panel B shows a low power view of the striatum in the E18.5 mouse telencephalon. The striatum is delineated by white arrows and stained diffusely for MOR, as is characteristic at this developmental stage. The mouse telencephalon was counterstained with DAPI. Lysates prepared from the dorsal cortex of E18.5 mouse brains were separated by LDS-PAGE, transferred to PVDF membrane, and then probed with a rabbit anti-MOR antibody (lanes 2 and 3). Immunoreactive bands corresponding to MOR (45 kDa) and the glycosylated MOR (70 kDa) are indicated. Lane 1 contains pre-stained MW standards (panel C). Panel D shows a higher power view of the mouse neonatal striatum (P6). MOR immunofluorescence appears patchy, as opposed to the diffuse staining seen in late foetal striatum. Patchy regionalisation (shown by white arrows) is characteristic of opioid peptides and opioid receptors during early neonatal development of the murine striatum. Scale bars: $100 \mu \mathrm{m}$. 


\subsubsection{MOR Staining and PCNA Staining}

In the E18.5 brain MOR IR was found within the ventricular neuroepithelium of the LV (Fig. 3.3, panels A-D), 3V (panel E), and Aq (panel F). PCNA is a marker for proliferating cells and cells that have recently divided, allowing regions of proliferative activity to be identified. PCNA IR can be seen in the nuclei of cells that line the wall of the LV, consistent with previous reports (Ajioka et al., 2006). PCNA-positive cells extend into the cortical neuroepithelial layer that covers the developing striatum (panels A and C). Importantly, we show in Fig. 3.3, panels A-D, that MOR IR is expressed in the same location as the PCNA IR, and that these MOR-positive and PCNA-positive cells extend along the cortical neuroepithelium (panels B and D). Panels E and F show regions of the 3V and Aq, respectively, which, similar to the LV, express MOR and PCNA at the ventricle wall. 


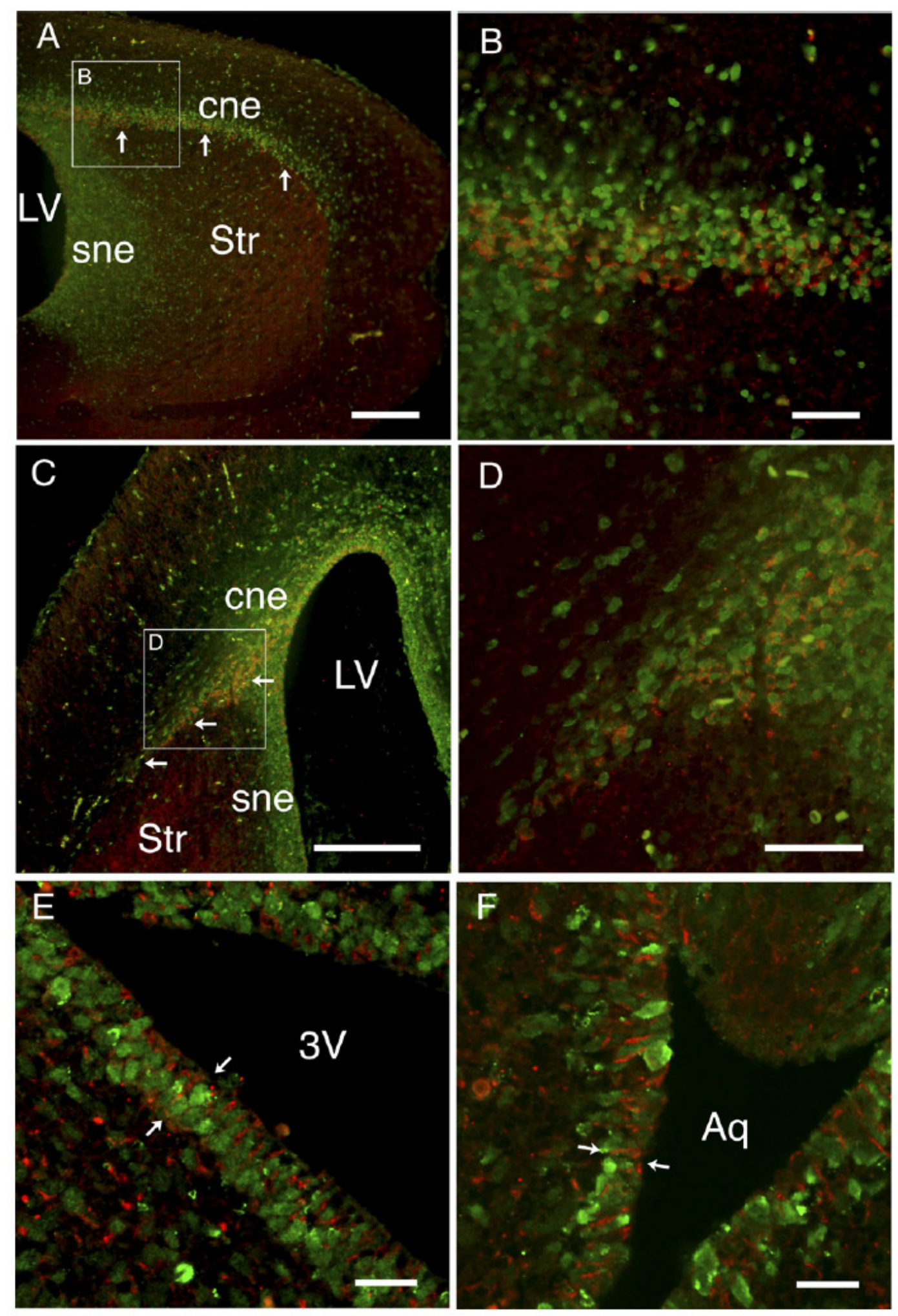


Fig. 3.3. MOR and PCNA IR in the E18.5 neuroepithelia of the LV, 3V, and Aq. Panels A and C show MOR IR (red) in coronal and sagittal sections of the LV, respectively. Proliferating cells (PCNA-positive) were stained (green). The cortical neuroepithelium (cne) in panels A and C is indicated by the white arrows. Higher magnification images of the boxed regions are presented in panels B and D and show that MOR IR was localised to the same area as PCNA IR. Panel E shows a confocal image of a coronal section of the ventral 3V in which MOR IR was tightly associated with a periventricular band of PCNA staining cells (located between the white arrows). Similarly, the proliferative zone of the Aq wall (white arrows, F) contained many PCNA IR nuclei and MOR IR cells. Scale bars: $200 \mu \mathrm{m}$ (A and C), $50 \mu \mathrm{m}$ (B and D), and $20 \mu \mathrm{m}$ (E and F).

\subsubsection{MOR and BrdU Staining in the $V Z$}

To examine the relationship between MOR expression and proliferation in detail, a more defined cohort of proliferating cells was labelled with BrdU. BrdU is only incorporated into cells undergoing DNA synthesis (cells in S-phase of the cell cycle). Dual-staining for BrdU and MOR was examined in the VZ of the LV in E15.5 and E18.5 mouse brains (Fig. 3.4). Confocal imaging of the E15.5 brains (panel A) showed that most of the BrdU stained nuclei (green) in the VZ were also MOR-positive (red). Examples of double-labelled cells are indicated by arrows. As not all of the MOR-positive cells were in S phase, many did not stain for BrdU. At the VZ in the E18.5 brains, fewer BrdU-stained nuclei were detected (Fig. 3.4, panel B), consistent with the decreased level of corticogenesis at this later developmental stage. Examination of orthogonal views by confocal microscopy (side bars) showed that the cytoplasm surrounding most BrdU-positive nuclei was MOR-positive (arrowheads) even when the $\mathrm{x}, \mathrm{y}$ view suggested the nuclei were negative for MOR staining, as is the case for the cell in Fig. 3.4, panel B marked by an asterisk. 

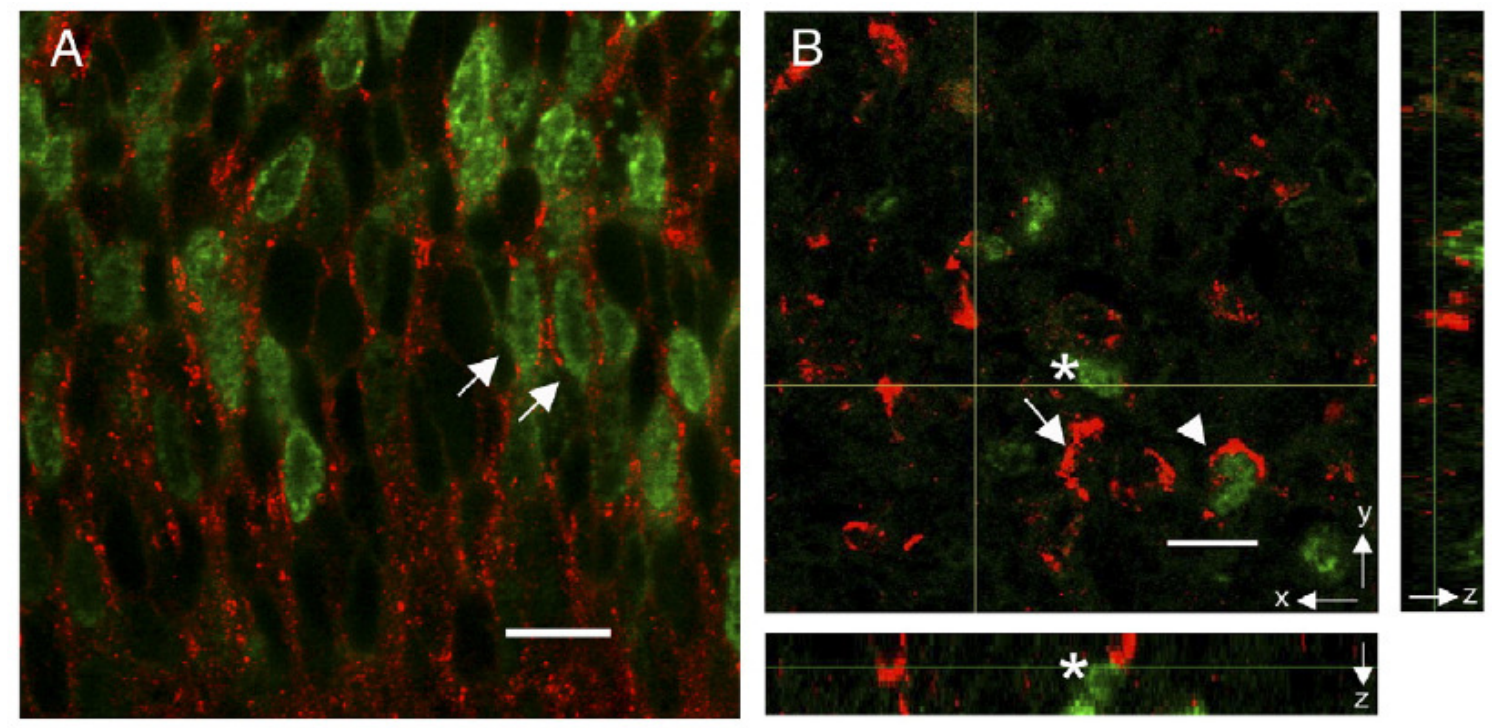

Fig. 3.4. MOR and BrdU IR in the E15.5 and E18.5 VZ. Panels A and B show confocal images of the VZ in E15.5 and E18.5 mouse brains, respectively. Panel A shows that most of the BrdU-positive nuclei (green) were found in MOR-positive cells (red) in the E15.5 brain. Examples of dual-labelled cells are indicated by arrows. Panel B shows the same region of brain in an E18.5 brain similarly stained. Orthogonal views (zplane transects at the yellow lines) are presented, and the $\mathrm{x}, \mathrm{y}$-plane comprising the main panel is indicated within the z-planes as green lines. Within the $\mathrm{x}$, y-plane, a BrdU-positive/MOR-positive cell (arrowhead) and a BrdU-negative/MOR-positive cell (arrow) are indicated. A cell which appears to have stained negative for MOR within the $\mathrm{x}, \mathrm{y}$ plane but can be seen to be MOR-positive within the $\mathrm{y}, \mathrm{z}$-plane is indicated by an asterisk (*). Scale bars: $10 \mu \mathrm{m}(\mathrm{A}$ and $\mathrm{B})$.

\subsubsection{Proliferation in the Developing Cerebellum}

The proliferative zone of the developing cerebellum is the EGL, located at the periphery of the cerebellum and densely packed with proliferating cells that stained strongly for PCNA (Fig. 3.5, panel A). In contrast to the LV, 3V, and Aq, MOR IR was not observed within 
the proliferative zone of the foetal cerebellum (Fig. 3.5, panels B and C) but was present, however, in the Purkinje layer. As the high density of cells within the developing cerebellum makes interpretation of immunohistochemical staining of PCNA IR difficult, cells in S phase were labelled by BrdU incorporation. E18.5, P6, and P12 pups were given BrdU (50 mg/kg) $1 \mathrm{~h}$ prior to sacrifice, and the cerebellum was stained for MOR IR and BrdU incorporation. Figure 3.6, panel A shows that the EGL of the E18.5 cerebellum contained many BrdU-positive nuclei (green) that did not stain for MOR (red); whereas, the developing Purkinje/molecular layer stained for MOR but contained few BrdU-positive nuclei. At P6 the Purkinje layer stained intensely for MOR, and the EGL contained abundant BrdU-positive nuclei with minimal MOR staining (panel B). By P12 (panel C), proliferation was much reduced, but MOR IR in the Purkinje layer was still clearly evident.
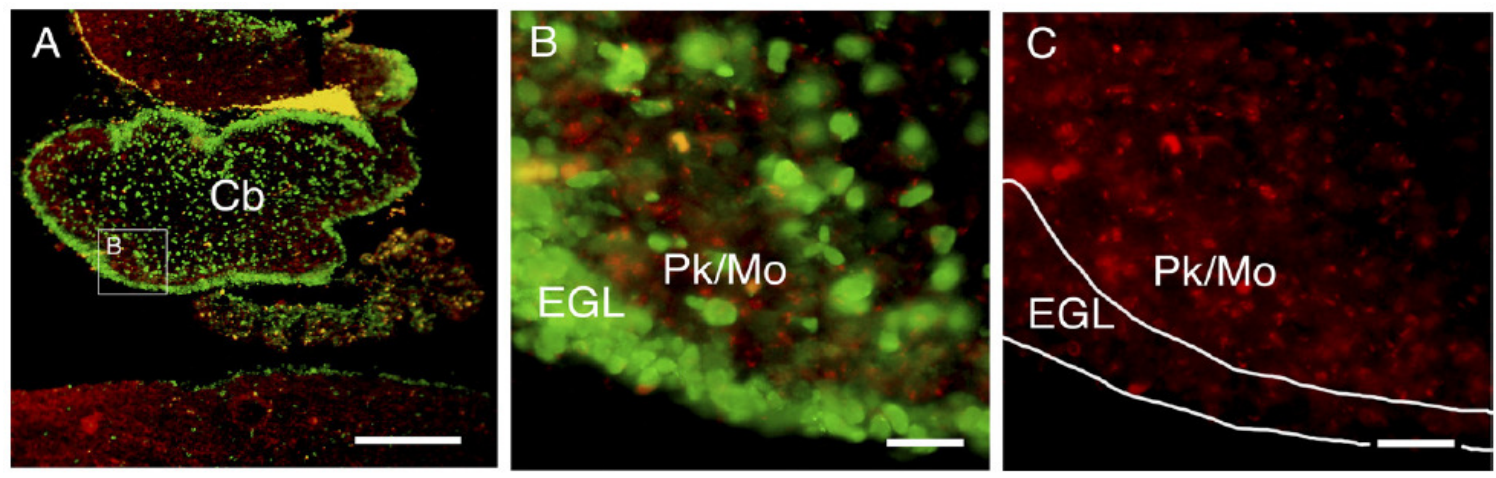

Fig. 3.5. PCNA and MOR IR in the E18.5 cerebellum. Panels A and B show both PCNA and MOR IR, while panel C shows only MOR IR. The EGL in panel A is clearly defined by intense PCNA staining (green) at the periphery of the cerebellum (panel A, low power, and panel B, high power). MOR IR (red) is seen within the Purkinje and molecular layers of the cerebellum just beneath the EGL (A and C). Little MOR IR, however, is present within the EGL, which is shown demarcated by the white lines in panel C. Scale bars: $200 \mu \mathrm{m}(\mathrm{A})$ and $20 \mu \mathrm{m}(\mathrm{B}$ and $\mathrm{C})$. 

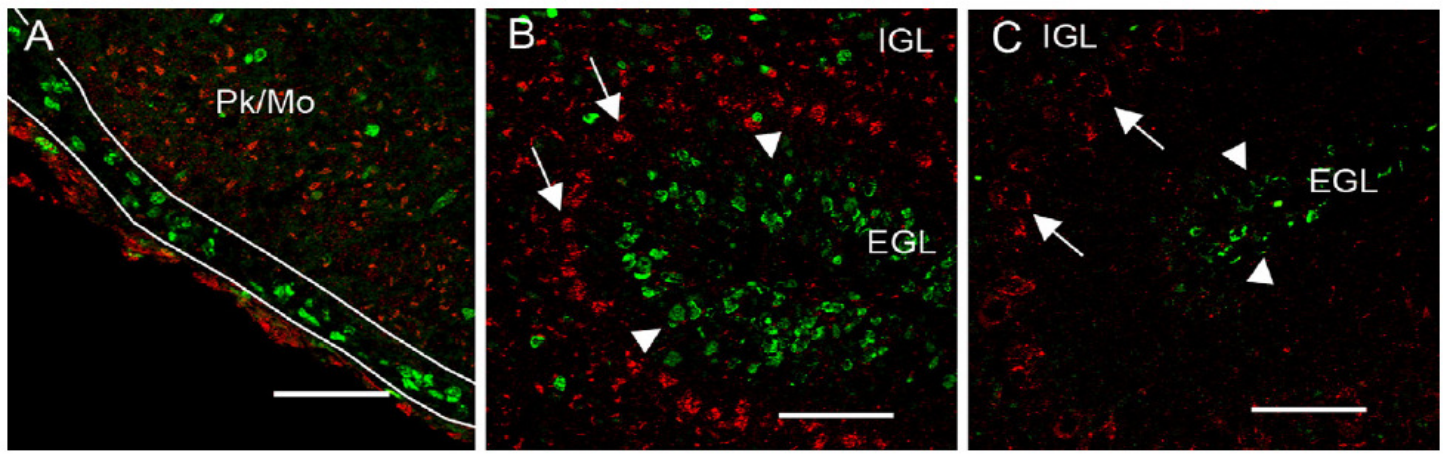

Fig. 3.6. Proliferation and MOR IR in the developing cerebellum. The E18.5 EGL was readily identified by its intense staining for BrdU (green, A, demarcated by white lines). MOR IR (red) was seen within the Purkinje and molecular layers $(\mathrm{Pk} / \mathrm{Mo})$ of the cerebellum just beneath the EGL. Little MOR IR, however, was present within the EGL. Panel B shows proliferation and MOR expression in the P6 cerebellum similarly stained. Strong MOR IR was present in Purkinje cells (white arrows) of the Purkinje layer, as well as the internal granule layer (IGL). Little MOR IR was seen within the EGL (indicated by the arrowheads) where most of the proliferative BrdU-positive cells were found. Similar staining patterns were seen in the P12 cerebellum (panel C) with the Purkinje cells being clearly defined as MOR-positive, but with decreased proliferation (BrdU-staining) in the EGL. Scale bars: $50 \mu \mathrm{m}$.

\subsubsection{MOR and GLAST Staining}

Radial glia are the neuronal progenitors of the cerebral cortex. They stained for GLAST

(Fig. 3.7, panels A-D, green staining) and had processes that extended from the LV wall to the pial surface to provide the scaffold on which pyramidal and interneurons migrate. Higher magnification confocal images of radial glial cell bodies at the LV wall in E15.5 (panel C) and E18.5 (panel D) brains showed that immunoreactivity for GLAST (green) and MOR (red) was located in the plasma membrane of cells. At E15.5 the VZ contains many cells that are both MOR IR and GLAST IR, although not all GLAST-positive cells 
stained for MOR. Orthogonal views confirming dual-labelling of a MOR-positive and GLAST-positive cell (indicated by arrows) are shown in panel C. Similarly, in the E18.5 VZ GLAST-positive cells that stained intensely for MOR (panel D) were clearly visible. Orthogonal views show that MOR was not evenly distributed within the cell membrane as is evident from cross-sections through the z-plane of the cell indicated by an arrow. Using a confocal approach, we found that as for the E15.5 VZ, not all GLAST-positive cells stained for MOR. To determine whether the progenitor cells in the neuroepithelium that overlies the striatum were also MOR and GLAST positive, sections encompassing the brain regions described in Fig. 3.3, panels A to D were stained. All of the MOR IR cells in this region were also GLAST-positive, however, these MOR IR and GLAST IR cells represented only a small fraction of the GLAST-positive population (Fig. 3.7, panels E and F). Even though GLAST-positive cells of the VZ expressed MOR, DOR IR could not be found (Fig. 3.8). The anti-DOR antibody was suitable for immunofluorescence in tissue, since the spinal trigeminal tract of a postnatal mouse stained brightly for DOR (panel C), as expected (positive control).

Confocal analysis of the $3 \mathrm{~V}$ and Aq walls (Fig. 3.9) revealed that the cells at the ventricle walls in these regions were also GLAST-positive (green) with long fibres that radiated perpendicularly (white arrows, panels $\mathrm{A}$ and $\mathrm{C}$ ). This suggests that these cells radiating out from the $3 \mathrm{~V}$ and Aq were also radial glia. Fig. 3.9 panels B and D show that some of these radial glia were also MOR IR (red), as indicated by arrows. 

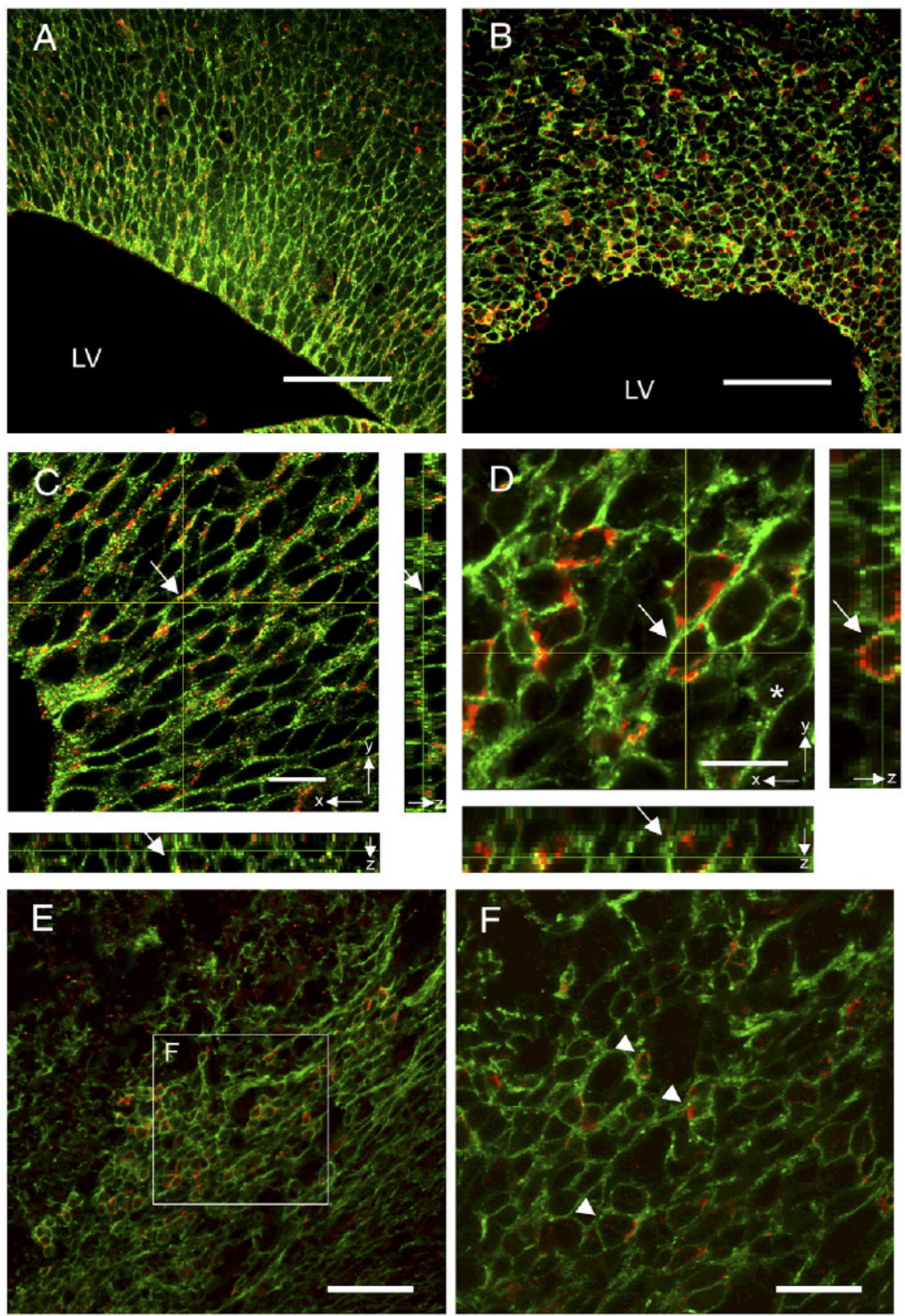
Fig. 3.7. MOR and GLAST IR in the E15.5 and E18.5 LV neuroepithelium. Panels A and B show low power images of the E15.5 and E18.5 VZ, respectively. Both panels show MOR IR (red) in the same region as GLAST staining (green). Panel C shows a higher magnification image of the E15.5 VZ in which MOR IR (red) and GLAST IR (green) can be seen within the same cell. Orthogonal views (z-planes) of the doublelabelled cell (indicated by the arrow) are presented in the side bars. Similarly, panel D shows a higher power image of the E18.5 VZ in which double-labelling for MOR and GLAST is more apparent. Nearly all the MOR-positive cells examined stained for GLAST, but many GLAST-positive cells did not stain for MOR. A GLAST-positive/MOR-negative cell is marked with an asterisk, and orthogonal views through a MORpositive/GLAST-positive cell (arrows) are presented in the side bars. Panels E and F show similarly stained regions of cortical neuroepithelium (equivalent to that in Fig. 3.3, panel D) from E18.5 brains in which MOR IR and GLAST IR are found within the same cell (arrowheads). Scale bars: $50 \mu \mathrm{m}$ (A, B and E), $20 \mu \mathrm{m}$ (F) and $10 \mu \mathrm{m}(\mathrm{C}$ and $\mathrm{D})$.
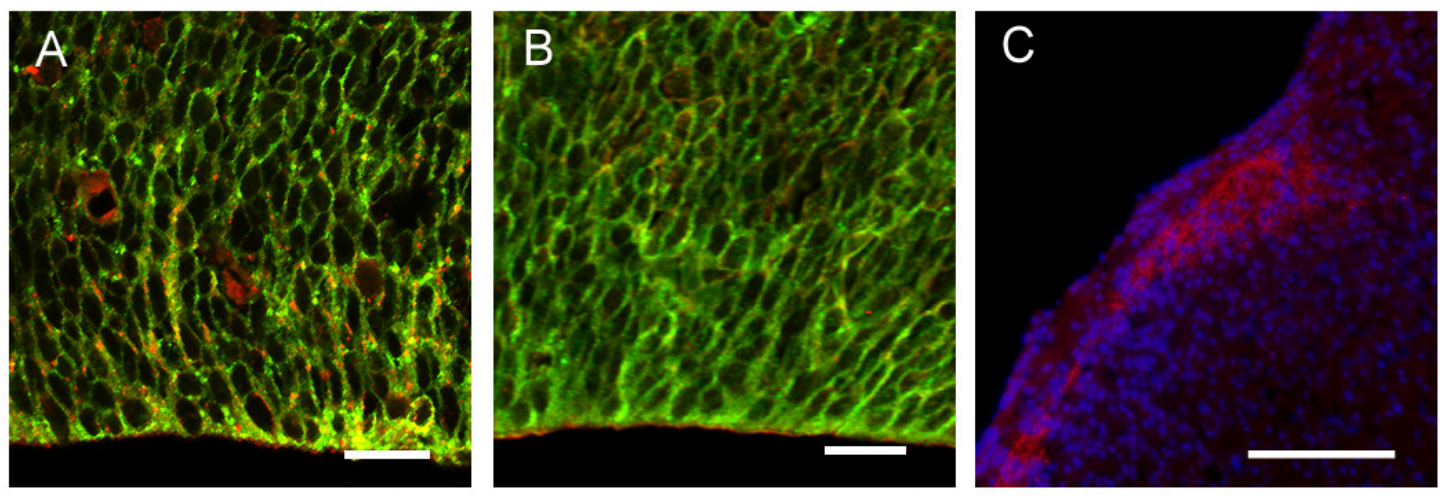

Fig. 3.8. DOR immunoreactivity in the E15.5 VZ. A confocal image of the E15.5 VZ shows radial glia stained for GLAST (green) (panel A). As in Fig. 3.7, panel C, these GLAST-positive radial glia also stained positive for MOR (red). However, when the same region of VZ is stained for DOR (red), there appeared to be minimal expression on the same GLAST-positive radial glia that also stained positive for MOR (panel B). Panel C shows a lower power photomicrograph of the spinal trigeminal tract, which stained strongly for DOR (red). DOR staining in the spinal trigeminal tract was performed under the same conditions as in panel B. The spinal trigeminal tract is counterstained with DAPI (blue). Scale bars: $20 \mu \mathrm{m}$ (A and B) and $200 \mu \mathrm{m}(\mathrm{C})$. 

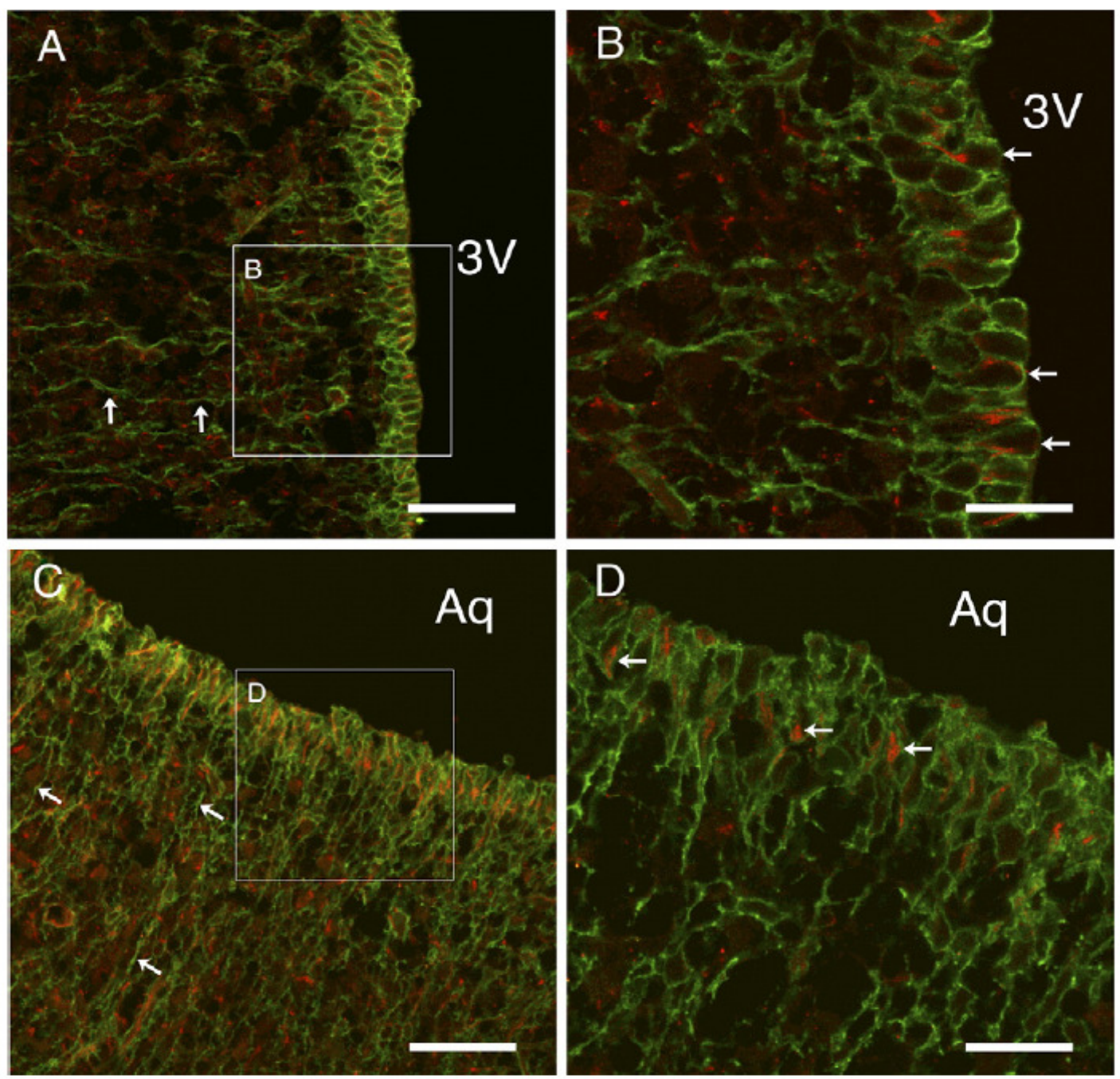

Fig. 3.9. MOR and GLAST IR in the neuroepithelia of the $3 \mathrm{~V}$ and Aq in the E18.5 mouse. Panel A shows intense GLAST staining (green) at the wall of the 3V. GLAST IR processes that extended perpendicular from the ventricular wall can be clearly seen (white arrows). MOR IR (red) was also located around the 3V (A and B). White arrows in panel B identify some of the cells that stained for both MOR and GLAST. Panel C shows MOR and GLAST IR at the wall of the Aq. Similarly, GLAST IR processes extended perpendicular from the wall of the Aq (C, white arrows) with many of the GLAST IR cells also expressing MOR (D, white arrows). Scale bars: $50 \mu \mathrm{m}$ (A and C) and $20 \mu \mathrm{m}$ (B and D). 


\subsubsection{MOR and DCX Staining}

To determine if the MOR IR seen in the cortex was expressed on migrating neurons, sections encompassing the regions described in Fig. 3.3 were double-stained for MOR and the neuronal migration-specific marker DCX. Fig. 3.10, panel A shows MOR IR (red) and DCX IR (green) around the LV with MOR IR being predominantly localised to the edge of the LV wall (arrowheads). Confocal imaging showed that the vast majority of MOR IR cells were not DCX IR. Panels B and C show confocal images of a region of the neuroepithelium equivalent to that presented in Fig. 3.3, panels B and D. As for the LV wall, the MOR IR cells of the cortical neuroepithelium did not express DCX, with MOR IR (red) and DCX IR (green) being mutually exclusive for the majority of cells (panel B, arrowheads). Panel C shows a higher-magnification confocal image of two DCX-positive cells (asterisk) in the cortical neuroepithelium. Orthogonal views confirm that these cells do not express MOR, although they are in close proximity to MOR IR processes.
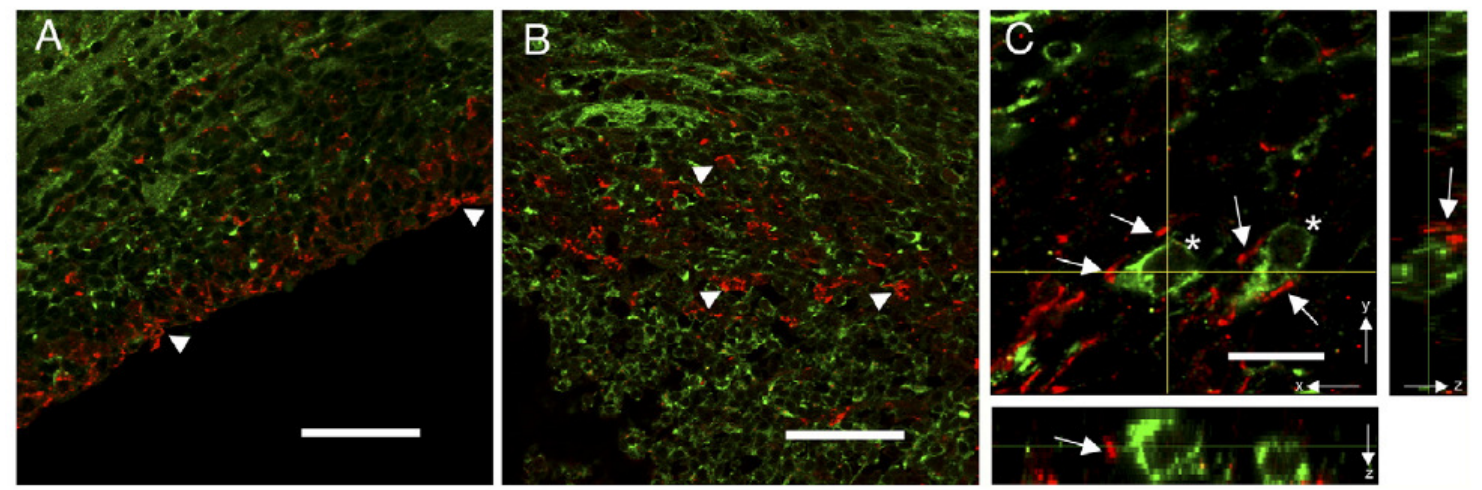

Fig. 3.10. MOR and DCX IR in the E18.5 neocortex. Panel A shows a region of VZ at the LV wall in an E18.5 brain stained for MOR (red) and DCX (green). MOR IR is located mainly within the VZ at the LV wall, with few dual-labelled MOR IR/DCX IR cells apparent. Panel B shows an equivalent region of cortical 
neuroepithelium to that presented in Fig. 3.3, panel D. Most of the MOR IR cells do not express DCX (arrowheads). Orthogonal views (panel C) are presented in the side bars of MOR-positive cells that confirm the absence of DCX IR within these cells. Panel C shows a higher magnification image of cells presented in panel B. Two DCX positive cells in the $\mathrm{x}, \mathrm{y}$-plane are indicated with an asterisk, and orthogonal views in the z-plane are presented in the side bars. Cytoplasmic staining for DCX (green) is clearly visible, and MOR IR (red) processes can be seen contacting the cell bodies (arrowheads). The orthogonal views confirm that the MOR IR seen adjacent to the cell bodies is not within the DCX-positive cell. Scale bars: $50 \mu \mathrm{m}(\mathrm{A}$ and B) and $10 \mu \mathrm{m}(\mathrm{B})$.

\subsubsection{Gradient of Proliferation and MOR Expression within the LV Cortical}

\section{Neuroepithelium}

Previous studies have shown that proliferation is not uniform throughout the cortex, with distinct gradients occurring both medial-lateral and rostral-caudal at early and mid-stages of corticogenesis (Miyama et al., 1997). We therefore wished to examine the topology of proliferation at the end of corticogenesis (E18.5) in the mouse. Proliferative cells were labelled with BrdU and labelled nuclei counted using a stereological approach on paraffinembedded sections. BrdU-stained nuclei were much more numerous at the anterior region of the LV than the posterior such that an anterior to posterior gradient of proliferation existed in the E18.5 mouse cortex (Fig. 3.11). A significant linear regression was fit to the data collected from three mouse embryos $(\mathrm{n}=3$ separate litters $)\left(\mathrm{R}^{2}=0.82, \mathrm{P}<0.05\right)$. The density of MOR IR cells was determined by counting fluorescently stained cells using a photomicroscope. Cell counts for MOR IR were expressed in Fig. 3.11 as the number of cells per unit area. The density of MOR IR followed the opposite trend to that for BrdU 
labelling, with MOR expression decreasing in a posterior to anterior manner, with the greatest density of MOR-positive cells in the posterior sections. A significant linear regression was fit to the data collected from three mouse embryos ( $\mathrm{n}=3$ separate litters) $\left(\mathrm{R}^{2}=0.91, \mathrm{P}<0.05\right)$

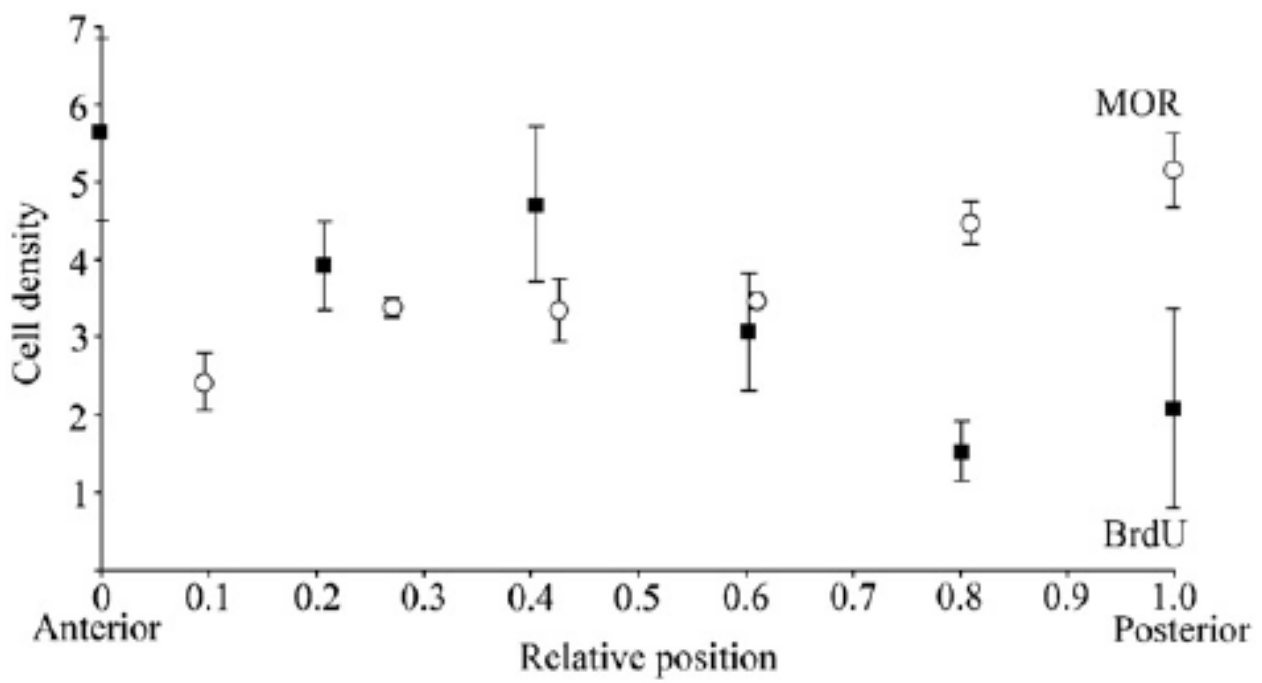

Fig. 3.11. Gradients of proliferation and MOR expression in the dorsal VZ of the LV in E18.5 mice. BrdU incorporation decreased in an anterior to posterior direction $(\mathrm{P}<0.05)$ (black squares), while the number of MOR IR cells (empty circles) increased anterior to posterior $(\mathrm{P}<0.05)$. Staining for BrdU incorporation was performed on paraffin-embedded sections and counted stereologically; whereas, MOR IHC was performed on fixed, then cryopreserved, $20 \mu \mathrm{m}$ sections using non-stereological methods. To correct for the effects of tissue shrinkage that occurs with paraffin embedding, the relative distance from the first anterior appearance of the LV (set arbitrarily at 0 units) to its disappearance posteriorly (1 unit) is plotted along the $\mathrm{x}$-axis. The y-axis represents the density of BrdU-stained nuclei $\left(\mathrm{mm}^{-3} \times 10^{5}\right)$ and MOR-stained cells $\left(\mathrm{mm}^{-2} \times 10^{3}\right)$. Values represent the mean \pm S.E.M. ( $\mathrm{n}=3$ separate litters). $\mathrm{P}$ values were determined by regression analysis. 


\subsection{Discussion}

The present study demonstrated that MOR is expressed within the cortical neuroepithelium of the E15.5 LV, and the neuroepithelia of the LV, 3V, and the Aq of the E18.5 mouse brain. The cells in these regions incorporate BrdU, and hence are proliferative. MOR IR cells within ventricular neuroepithelia were also GLAST IR indicating that they are radial glia. MOR IR cells in proliferating regions were not DCX IR, suggesting that the MORpositive cells within neuroepithelium are radial glial progenitor cells and not migrating daughter neuroblasts. The same staining pattern was seen at all the ventricular surfaces examined.

\subsubsection{Antibody Validation}

While validating the rabbit anti-MOR antibody, western blots were performed to identify detected proteins according to molecular weight. Western blotting using the anti-MOR antibody (AB5511) detected two bands, one at $45 \mathrm{kDa}$ and the other at $70 \mathrm{kDa}$. These bands correspond to the unprocessed MOR protein and the glycosylated form, respectively. Several studies support the existence of MOR glycosylation (see Table 3.1) at similar molecular weights (Eppler et al., 1993; Liu-Chen et al., 1993; Ding et al., 1996; ChaleckaFranaszek et al., 2000; Christoffers et al., 2003; Kivell et al. 2004). Also, the ExPASy Proteomics Server identifies 4 potential glycosylation sites on the murine MOR protein (http://ca.expasy.org/uniprot/P42866). 


\begin{tabular}{|c|c|c|c|}
\hline Species & Detection method & $\begin{array}{l}\text { Detected size of opioid } \\
\text { receptor protein }\end{array}$ & Study \\
\hline Rat & $\begin{array}{l}\text { Isolated using biotinylated } \\
\text { beta-endorphin }\end{array}$ & $\begin{array}{l}60-70 \mathrm{kDa} \text {, reduced to } 40 \\
\mathrm{kDa} \text { with glycosylase. MOR } \\
\text { protein sequence isolated. }\end{array}$ & Eppler et al., 1993 \\
\hline Mouse & $\begin{array}{l}\text { Detected with }\left[{ }^{3} \mathrm{H}\right] \text { beta- } \\
\text { FNA }\end{array}$ & $\begin{array}{l}66 \mathrm{kDa} \text {, reduced to } 43 \mathrm{kDa} \\
\text { with glycosylase }\end{array}$ & Liu-Chen et al., 1993 \\
\hline Rat & $\begin{array}{l}\text { Detection with three } \\
\text { different antibodies }\end{array}$ & A diffuse band at $\sim 60 \mathrm{kDa}$ & Ding et al., 1996 \\
\hline Rat & $\begin{array}{l}\text { Two antisera raised against } \\
\mathrm{C} \text { and } \mathrm{N} \text { termini of MOR }\end{array}$ & Band spanning $60-75 \mathrm{kDa}$ & $\begin{array}{l}\text { Chalecka-Franaszek et al., } \\
2000\end{array}$ \\
\hline Mouse & $\begin{array}{l}\text { Modified Oprm gene to } \\
\text { include hexahistidine tag } \\
\text { and FLAG tag }\end{array}$ & $\begin{array}{l}\text { Glycoprotein found at } 80 \\
\text { kDa. MOR protein sequence } \\
\text { identified with MALDI-MS }\end{array}$ & Christoffers et al., 2003 \\
\hline Rat & $\begin{array}{l}\text { Commercially available } \\
\text { Chemicon antibodies } \\
\text { (AB1774 and AB1580) }\end{array}$ & Two bands at 50 and $70 \mathrm{kDa}$ & Kivell et al., 2004 \\
\hline
\end{tabular}

Table 3.1: Studies that have reported glycosylated forms of the MOR. 


\subsubsection{Implications of MOR Localisation}

\subsubsection{MOR in the VZ and the SVZ of the E15.5 and E18.5 Mouse}

MOR expression in the VZ of the E15.5 mouse dorsal telencephalon has implications for both neurogenesis and gliogenesis during construction of the cerebral cortex, as well as corroborates immunohistochemical results from the study of Reznikov et al. (1999). The presence of MOR on radial glia means that direct opioid signalling may impact upon developmental processes. Expression of opioid system receptors and ligands on cells of astrocytic lineage (Vilijn et al., 1988; Hauser et al., 1990; Ruzicka et al., 1995; StieneMartin et al., 1998) perturbs both proliferation and differentiation in vitro (Stiene-Martin et al., 1991b; Stiene-Martin et al., 1993; Hauser et al., 1996).

Interestingly, no DOR expression was found in the cortical VZ of the E15.5 mouse. This result contrasts with that of Reznikov et al. (1999) who found diffuse DOR staining in the VZ. Other studies, however, have demonstrated that in contrast to MOR and KOR, DOR expression is generally very low in the foetal rodent brain (Leslie et al., 1998; Zhu et al., 1998).

Intense MOR expression on PCNA-positive cells of the dorsal-rostral (Fig. 3.3, panel B) and dorsal-lateral (panel D) SVZ of the E18.5 mouse was surprising, given past radio in situ hybridisation studies (Leslie et al., 1998; Zhu et al., 1998). However, based on older radioligand binding studies that found signal within the developing SVZ (Kent et al., 
1982), as well as literature that documents the sensitivity of neonatal astrocytes to opiates (c.f. literature published from the groups of Hauser and Coscia), this result was consistent with a significant number of previous studies (see above).

The extensions of the SVZ in the late foetal mouse brain observed in Fig. 3.3, panels A-D, are a result of the fusion of the lateral ventricle at the cortico-striatal boundary. The rostral regions of SVZ (Fig. 3.3, panels A and B) produce cells for the rostral migratory stream in the adult brain. The dorsal-lateral regions of SVZ shown in panels $\mathrm{C}$ and $\mathrm{D}$ are a rich source of glia in the developing postnatal brain (reviewed in Brazel et al., 2003). Astroglia from the SVZ are a source (albeit a very minor one) of cortical neurons (Ganat et al., 2006) as well as neurons for the ventral forebrain in the postnatal rodent (De Marchis et al., 2004). As well as being a proliferative area in the neonate, the SVZ retains neurogenic capability in the adult mammal (Lois and Alvarez-Buylla, 1993) and contributes to increases in cell number (partly due to increased proliferation) in response to injury in the rat cerebral cortex. Interestingly, after injury in the brain, neurons migrate to the area of injury (reviewed in Romanko et al., 2004). Thus the expression of the MOR on astrocytic lineage cells of the SVZ is potentially of wider interest to areas of neuro-repair and regeneration in the adult brain.

The existence of an anterior to posterior gradient of proliferation within the proliferative zone of the E18.5 dorsal forebrain and an inverse gradient of MOR expression is consistent with the idea of MOR activation being anti-proliferative. It is, however, speculative to suggest that MOR signalling maintains or regulates the gradient of proliferation in vivo, as 
there is still little direct supporting evidence to indicate that opioid ligands have an antiproliferative effect in vivo via MOR signalling within the context of the perinatal rodent forebrain (Stiene-Martin et al., 2001). The data we have presented in this study does, however, place expression of the receptor on the appropriate cells for modulation of corticogenesis, and it is of interest to note that morphine exposure through the period E12 to P6 in the rat decreased cortical neuronal density (Seatriz and Hammer, 1993).

\subsubsection{MOR in the Developing Cerebellum}

In contrast to the epithelia lining the ventricular walls, the proliferative zone (EGL) of the developing cerebellum lacked MOR immunoreactivity in the E18.5 brain. The cerebellum is a late-developing structure and is extremely immature in the late foetal mouse (Goldowitz and Hamre, 1998). We, and others, have conclusively shown that the rodent cerebellum expresses MOR mRNA and protein, predominantly in the Purkinje layer and the deep cerebellar nuclei (Mrkusich et al., 2004; Xu et al., 2006, Zhang et al., 2006). Mrkusich et al. (2004) demonstrated the presence of opioid receptor mRNA as well as protein, via immunohistochemistry, in Purkinje cells and the deeper cerebellar nuclei. This was also demonstrated immunohistochemically by Zhang et al. (2006). Xu et al. (2006) corroborated these findings in mice using a green fluorescent protein-tau reporter under control of the exon 1 MOR promoters by demonstrating MOR promoter activity in Purkinje cells as well as the deeper cerebellar nuclei. Interestingly, several years earlier, the presence of opioid receptor expression in the cerebellum was discounted by both radio in situ 
hybridisation studies as well as immunohistochemical studies (Mansour et al., 1994; Mansour et al., 1995; Ding et al., 1996; Zhu et al., 1998).

Given the relative immaturity of the cerebellum at birth and its continued development up to the third postnatal week (Goldowitz and Hamre, 1998), we reasoned that MOR expression may increase after E18.5. This was, however, not the case since the P6 and P12 cerebellum showed strong MOR staining of Purkinje cells, as in the adult, but replicating (BrdU-positive) cells still lacked MOR expression in the postnatal brain. These data suggest a different role for MOR signalling in the developing cerebellum than that in the ventricular neuroepithelia. The data also corroborate our previous report that MOR mRNA is expressed in Purkinje cells of the developing cerebellum (Mrkusich et al., 2004).

The lack of MOR staining in the EGL of the cerebellum was surprising, given findings published in the literature (Hauser, 1992; Hauser et al., 2000). Hauser (1992) reported that morphine treatment inhibited BrdU incorporation in neuroblasts from the $\mathrm{EGL}\left(\mathrm{IC}_{50}=22.5\right.$ $\mathrm{nM}$ ). The inhibitory effect of morphine exposure was shown again in vitro, along with MOR immunostaining on isolated neuroblasts from the EGL. As the anti-MOR antibody used in the current study has no difficulty in detecting MOR in Purkinje cells, the striatum, and in dorsal horns of the spinal cord, MORs in the EGL may have been expressed at such a low level that our detection system was not sensitive enough to find them. Alternatively, EGL neuroblasts may have up-regulated MOR as a consequence of these cells being cultured in vitro in the studies of Hauser (1992) and Hauser et al. (2000). 


\subsubsection{Technical Considerations}

It should be noted that the antibody used in this study (Chemicon, rabbit anti-MOR, AB5511) was raised against exon 4 of MOR. Exon 4 containing splice variants of the MOR include MOR-1, as well as the exon-11 containing splice variants MOR-1H to MOR-1L (Abbadie et al., 2004). However, this antibody does not recognise the rodent carboxy terminus MOR splice variants such as MOR-1A to MOR-1F. As these MOR carboxy terminus splice variants differ in their responses to endogenous opioid peptides as well as exogenous opiates (Bolan et al., 2004; Pasternak et al., 2004), future work is needed to characterise the foetal expression patterns of these variant receptors in relation to MOR expression. We have previously detected mRNA coding MOR in the developing rat cerebellum (Mrkusich et al. 2004). This study used riboprobes that detected the 5' UTR of MOR mRNA. Using the anti-MOR antibody described in this study, we found good correlation between MOR mRNA and protein expression in all regions of the cerebellum apart from the EGL. As others have shown differential immunohistochemical localisation for carboxy terminus splice variants in the adult rat CNS (Abbadie et al., 2000), it is possible that the discordance between protein expression and mRNA localisation in the EGL may be attributable to expression of a splice variant lacking exon 4. Alternatively, the discrepancy may simply reflect the lower resolution of mRNA in situ hybridisation and the confounding factor of high cell density in the developing cerebellum that would reduce the signal-to-noise ratio. 
In the present chapter, MOR expression in proliferative neuroepithelia of the developing mouse brain was observed only on replicating radial glia (BrdU-positive, GLAST-positive cells) around the ventricular neuroepithelia, but not on newborn migrating neurons (DCXpositive cells). As there is evidence in the literature to support the hypothesis that MOR signalling modulates proliferation, particularly in astrocytes in vitro, the possibility of MOR modulation of cell cycle progression in the developing foetal brain warrants further investigation.

The results presented in this chapter have been published in Brain Research (Sargeant et al., 2007) 


\title{
4. MOR is Expressed on Glia of the Foetal Human Forebrain
}

\author{
4.1 Introduction
}

\subsubsection{The Need for Human Studies}

The wealth of data collected on the complicated relationship between opioids and cellular proliferation has been obtained mainly from rodent models. The little data that does exist on how opioids interact with human CNS progenitor cells has been obtained from studies using dissociated tissue in culture (Sheng et al., 2007). Such studies are limited as valuable information regarding spatial distribution, and how cells might interact with their surrounding cellular matrix, is lost. As the development of the rodent brain does subtly diverge from primate brain development in aspects other than sheer size and proportions, it is therefore important to gather data on the endogenous opioid system in vivo, in the developing human foetus.

4.1.2 Anatomical and Cellular Contrasts between Human and Rodent Corticogenesis 
The primary proliferative zone of the developing rodent forebrain is the VZ (Dehay and Kennedy, 2007). During the peak of neurogenesis in the mouse forebrain (E14 (Miyama et

al., 1997; Caviness et al., 2003)), the largest proliferative region is the pseudostratified VZ; whereas, the smaller SVZ is a zone where intermediate progenitor cells (basal progenitors) undergo terminal, neurogenic divisions. In contrast to the developing rodent cortex, the primate SVZ dwarfs the VZ at the equivalent developmental stage. Furthermore, the primate SVZ is split by the inner fibrous layer into the inner and outer SVZ (Smart et al., 2002). Another difference is the primate SVZ generates inhibitory interneurons (Letinic et al., 2002); whereas, in the rodent brain, this is primarily the role of the ganglionic eminences (Bulfone et al., 1993; Porteus et al., 1994; De Carlos et al., 1996; Lavdas et al., 1999). It has been suggested that enlargement of the primate SVZ is directly related to the evolutionary enlargement of the primate cerebral cortex (Zecevic et al., 2005).

\subsubsection{Cell Cycle Ontogeny}

As well as these anatomical and functional differences between the proliferative zones of the primate and rodent, cell cycle kinetics are also markedly altered. In the rodent forebrain, there is a consistent increase in cell cycle time as development progresses (Miyama et al., 1997), whereas in the primate the neurogenic interval increases from the onset of neurogenesis until mid-corticogenesis after which there is a large decrease in cell cycle time at the commencement of genesis of layer III of the cerebral cortex (Kornack and Rakic, 1998, see Chapter 1). Irrespective of the differences between rodent and primate corticogenesis, both the positioning and occurrence of non-ventricular surface mitoses at 
the preplate stage of corticogenesis (very early in development) are remarkably similar (Carney et al., 2007). Also, the primate SVZ, like the rodent SVZ, is heavily involved with generation of supragranular layer neurons (Tarabykin et al., 2001; Lukaszewicz et al., 2005)

\subsubsection{Neuronal Progenitor Cell Types}

Radial glial cells were first identified as a scaffold upon which neurons migrated in the primate brain using GFAP immunohistochemistry (Rakic, 1972; Levitt and Rakic, 1980). Even though both murine and primate radial glia serve as a scaffold for migrating neurons, and neuronal progenitor cells (Mo et al., 2007), rodent radial glia do not express the intermediate filament GFAP until they differentiate into astrocytes (Tramontin et al., 2003). In addition, rodent radial glial nuclear movements are restricted to the VZ, whereas in the human, radial glial nuclei are free to migrate into the SVZ or the intermediate zone to undergo mitosis (Howard et al., 2006).

Furthermore, in the foetal human brain, neuronally restricted progenitor cells that express markers such as beta III-tubulin and DCX can also undergo mitosis to produce neurons (Howard et al., 2006; Mo et al., 2007). In spite of much research into rodent brain development, such cells have not yet been found in the developing mouse brain, although the recent discovery of short neuronal precursor cells in the foetal rodent brain (Gal et al., 2006) has provided a possible murine equivalent for human neuronally restricted precursors. 


\subsubsection{Rate of Evolutionary Change}

Primate genes involved with brain development and behaviour evolved significantly more quickly than their rodent counterparts over the same time (Dorus et al., 2004). In fact, genes involved with brain development and behaviour were the fastest evolving genes in the primate. Some of these genes included ASPM (abnormal spindle-like, microcephaly associated) and microcephalin, both of which regulate brain size in the human (Cox et al., 2006). Interestingly, Dorus et al. (2004) also showed that MOR gene had evolved significantly faster in the primate than in the rodent, suggesting that the MOR was part of the genetic shift that contributed to increased complexity in the human brain.

Although the developing primate and rodent brain have a lot of features in common, there are aspects of significant difference. This makes it important to assess whether results obtained in the rodent model can be extrapolated to the human developing cortex. Thus, the aim of the work presented in this chapter was to determine if the immunohistochemical findings from the mouse, that showed MOR expression on radial glia of the late foetal forebrain (Chapter 3), was also true for the human foetal brain.

\subsection{Research Design}




\subsubsection{Immunohistochemical Markers}

In order to assess MOR IR, the same antibody was used for the human studies as that used for the mouse studies. This was possible because the antigenic peptide used to generate the antibody (from the carboxy terminus of MOR) does not vary in peptide sequence between rodents and humans. Human radial glia were identified by staining for vimentin, a class III intermediate filament protein (Herrmann and Aebi, 2000), rather than GLAST as in the mouse. To further characterise MOR staining, nestin (NeuroEpithelial STem cell marker), a class VI (or class IV) intermediate filament, that is expressed by proliferating neuroepithelial and radial glial cells (Hockfield and McKay, 1985; Frederiksen and McKay, 1988) was also used. Nestin is expressed by neuronal progenitor cells (Frederiksen et al., 1988; Cattaneo and McKay, 1990). As in the mouse, DCX is expressed on migrating neurons.

\subsubsection{Tissue Processing and Image Acquisition}

All immunohistochemistry was performed on fixed-frozen, $40 \mu \mathrm{m}$ thick, coronal cryostatmicrotome sections. Images were captured using an Olympus FV1000 confocal system. Cell counting was performed on sections of human foetal forebrain within the dorso-lateral SVZ at the level of the caudate-putamen. Image manipulation and cell counting was performed using Fireworks (v7.0.2.295) and ImageJ (v1.37) software. 


\subsection{Results}

\subsubsection{Sample Fixation}

Human foetal brain samples were gifted from Associate Professor Tailoi Chan-Ling and transported from Sydney University in sterile PBS. Fig. 4.1 shows the brain samples on arrival. Brains were fixed prior to transport from Australia by a number of different methods that included either fixation in PFA or ethanol (Table 4.1).

\begin{tabular}{lll}
\hline Brain ID & Age $(\mathrm{GW})$ & Fixation Protocol \\
number & 12 & PBS containing $4 \%$ PFA overnight \\
\hline 1 & 16 & 8 min PBS containing $4 \%$ PFA, 40 min $70 \%$ ethanol \\
2 & 16 & PBS containing $4 \%$ PFA overnight \\
4 & 19 & 8 min PBS containing 4\% PFA, 40 min 70\% ethanol \\
5 & 20 & PBS containing 4\% PFA overnight \\
\hline
\end{tabular}

Table 4.1: Fixation parameters for the brain samples obtained from As. Prof. T.C-L.

Sectioning and Nissl staining of brain 1 (12 gestational weeks (GW)) showed it consisted almost entirely of brainstem (8.4, Appendix 4); however, all other brain samples contained regions of forebrain that could be used to investigate opioid receptor staining and its relationship with glial markers. Because human brain tissue that had been fixed overnight in $4 \%$ PFA gave inconsistent immunostaining results, it was decided to use the human brain 
samples that had been fixed in ethanol after $8 \mathrm{~min}$ in 4\% PFA for the rest of the analysis (brains 2 and 4).
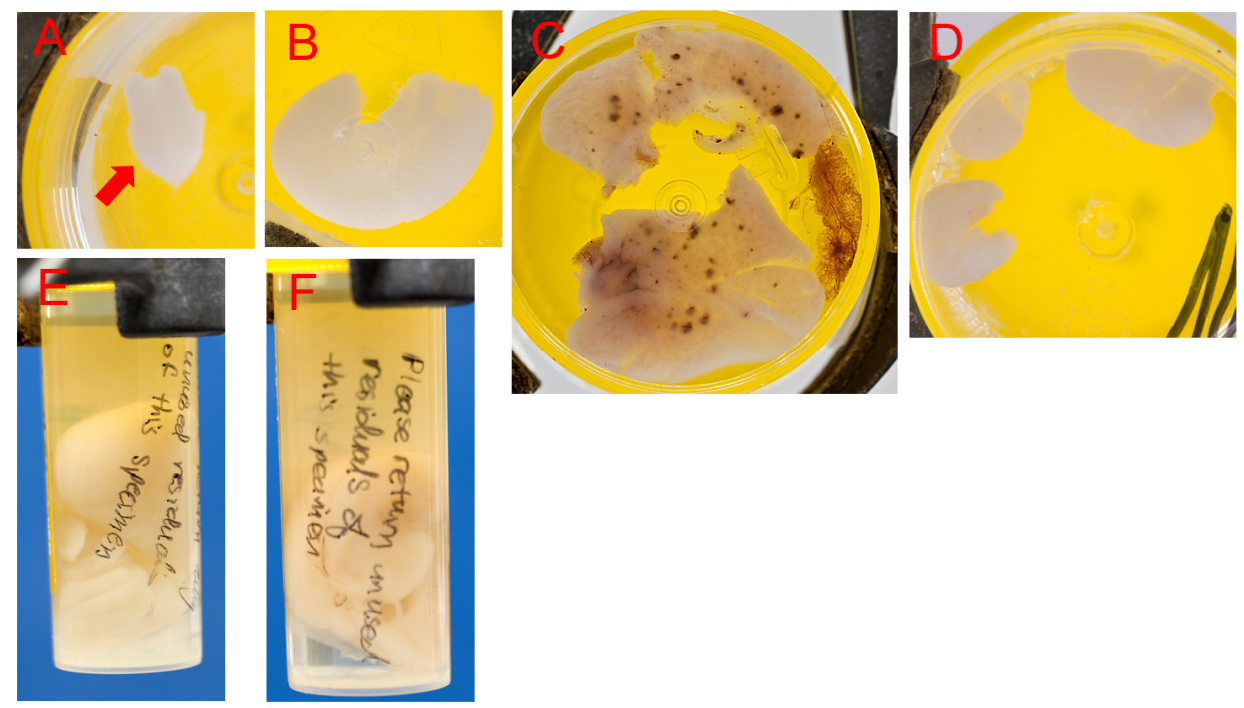

Fig. 4.1. Human foetal brain samples on arrival from Australia. Panel A shows brain 1, which was 12 gestational weeks (GW) (red arrow). Panel B shows brain 2, a $16 \mathrm{GW}$ brain that had been fixed in ethanol. A $16 \mathrm{GW}$ brain (brain 3) that had been fixed in 4\% PFA (panel C) demonstrated the different effects of fixation on gross sample morphology, as compared with panel D. Panel D shows a fragmented 19 GW brain (brain 4) that had been fixed in ethanol, and panels E and F show a $20 \mathrm{GW}$ brain that had been quartered before packaging and transport (brain 5).

\subsubsection{Human Foetal Brain Sample and Reconstruction}

Brain number 3 was obtained as a number of small fragments on arrival. The foetal brain was photographed and the image was reorganised to allow identification of brain regions (Fig. 4.2). 


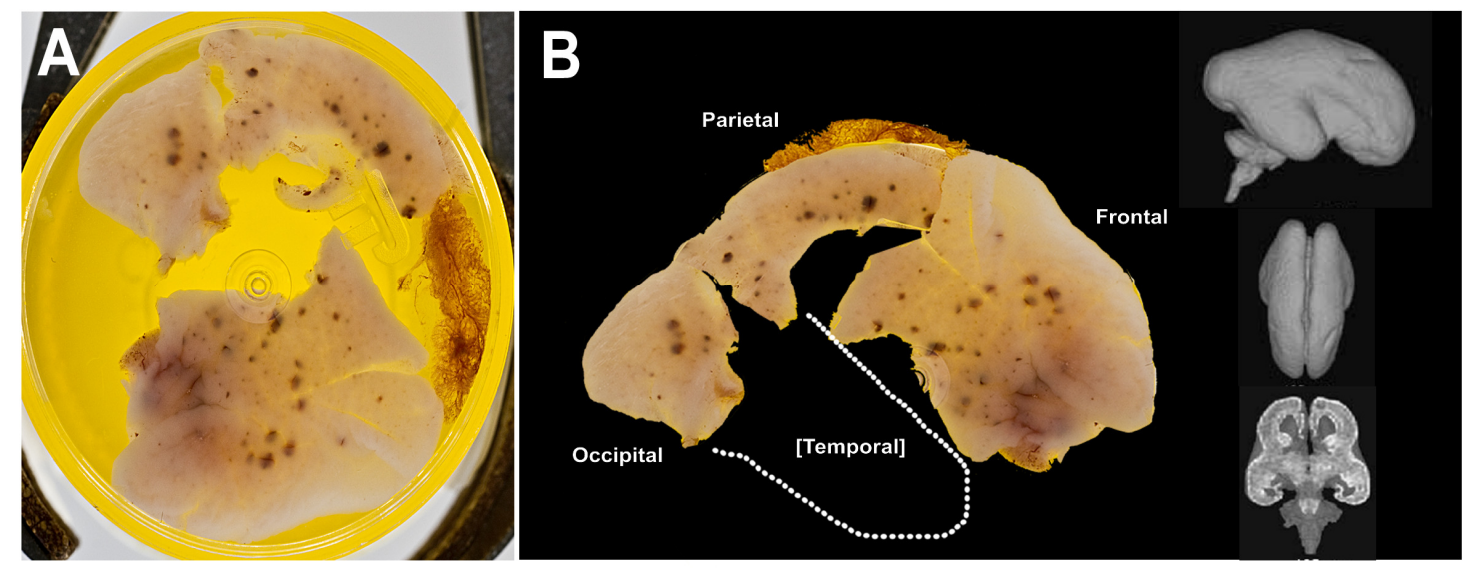

Fig. 4.2. Photography and identification of a main structures. Panel A shows the $16 \mathrm{GW}$ foetal brain (brain 3) on arrival in its sample container. Digital rearrangement of the photograph presented in panel A allowed for determination of anatomical aspects of the brain (panel B). On the right hand side of panel B are images created from MRI data taken from $16 \mathrm{GW}$ foetuses (Sbarbati et al., 2004). Subsequent microscopic morphology confirmed the rearrangement presented in panel B.

\subsubsection{Opioid Receptor Antibody Staining in the Human Brain}

Frozen tissue was sectioned from regions of forebrain that contained striatum. Ligand binding studies have shown the human striatum expresses both MOR and KOR (Vonkeman et al., 1996; Voorn et al., 1996; Daunais et al., 2001). When sections of human forebrain were stained with antibodies for MOR and KOR, fibre tracts within the striatum were clearly stained. This anatomy is in agreement with the known function and distribution of these opioid receptors in the striatum (Fig. 4.3, panels C and D). Omission of the primary opioid receptor antibodies resulted in no staining in adjacent sections of striatum, showing that staining was not due to non-specific binding by the secondary antibody (Fig. 4.3, panels A and B). 

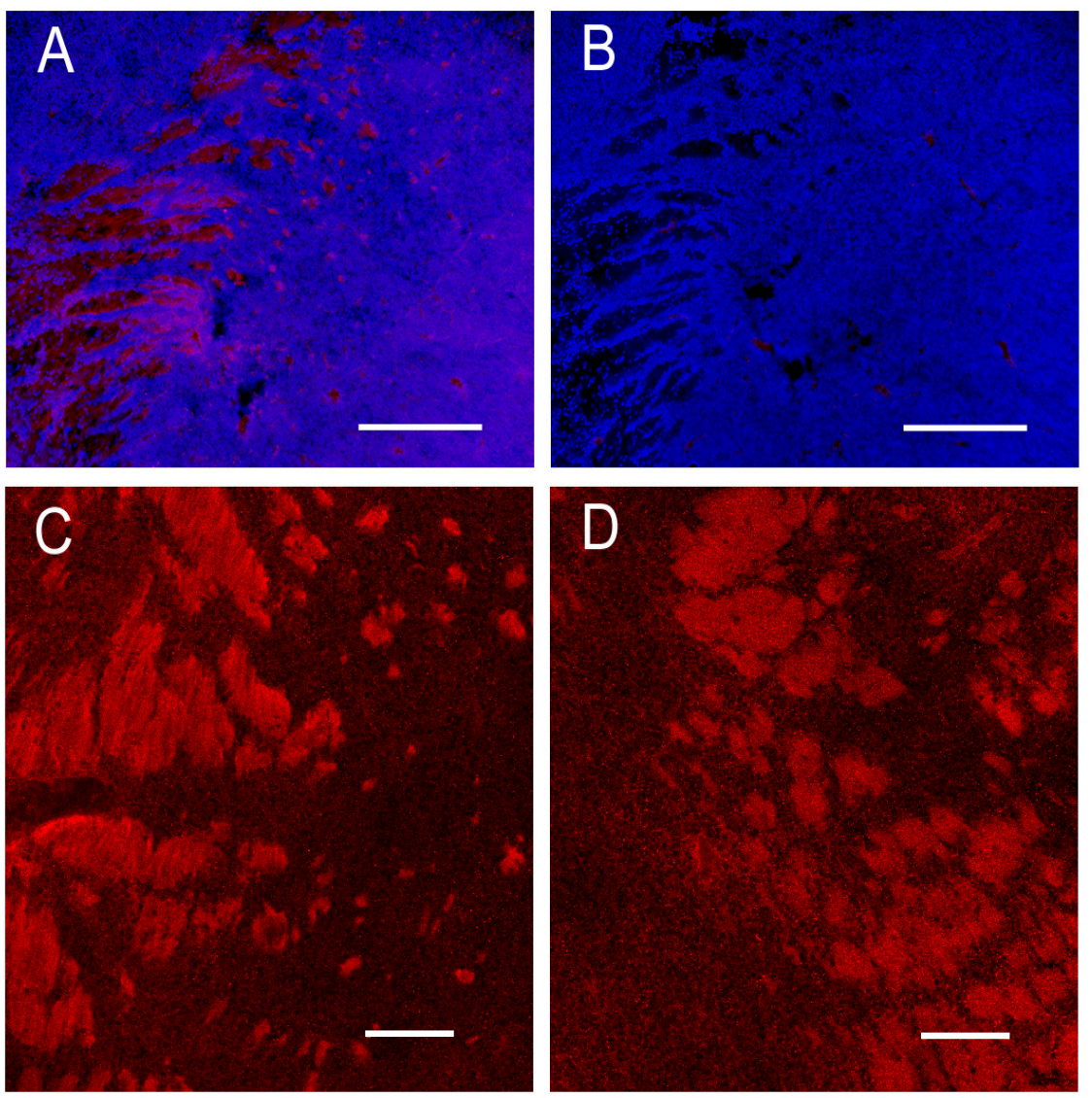

Fig. 4.3. Opioid receptor staining in the $16 \mathrm{GW}$ human striatum. Panel A shows MOR staining (red) and DAPI staining for nuclei (blue). Panel B shows an adjacent section of human striatum stained with only the goat anti-rabbit-Alexa 555 antibody. This image was captured and manipulated identically to that in panel A. Panel C shows MOR IR in the human striatum. Fibre tracts were clearly stained for MOR (red). Panel D shows the striatum of the same brain stained for KOR. This staining was similar to that of MOR. Scale bars: $200 \mu \mathrm{m}(\mathrm{A}$ and $\mathrm{B})$ and $100 \mu \mathrm{m}(\mathrm{C}$ and $\mathrm{D})$.

Upon investigation of the dorso-lateral VZ/SVZ, MOR-positive cells that had the same staining characteristics as in the late foetal mouse brain were identified. Interestingly, no such KOR-positive cells could be found in this region. Staining for KOR was not undertaken in the mouse. The MOR-positive cells present in the dorso-lateral SVZ of the 
16 and 19 GW human brain were present up to the ventricular edge (Fig. 4.4, panels A and B); however, they were not present in great numbers in the VZ, as seen in the late foetal mouse brain (Fig. 4.4, panel C).
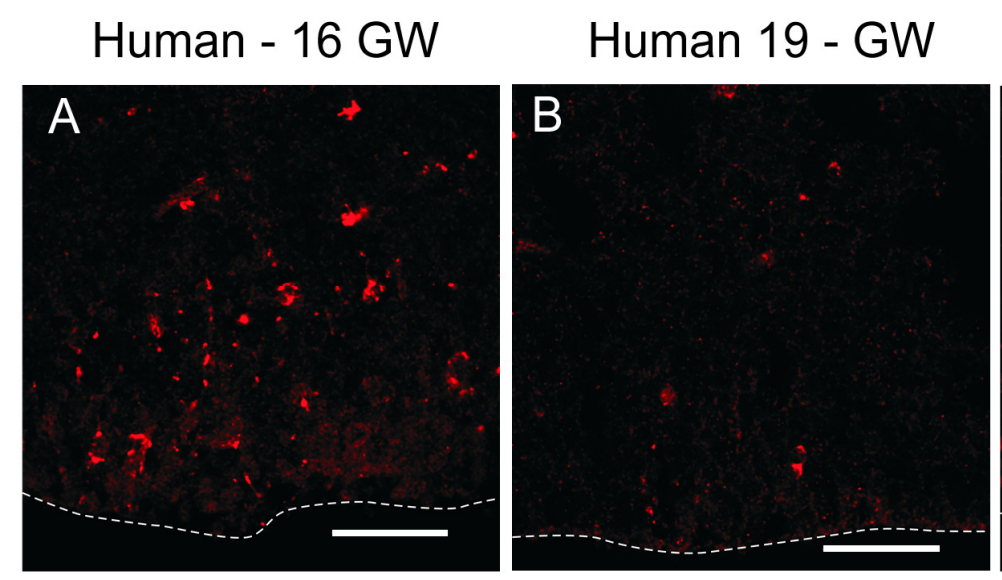

\section{Mouse - E18.5}

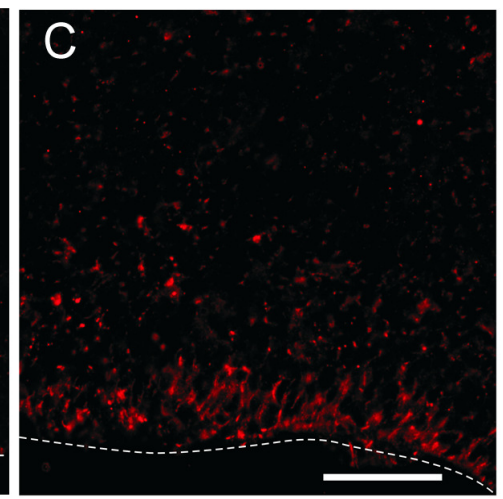

Fig.4.4. MOR staining at the LV surface in the human and in the mouse. Panels A and B show MOR staining at the lateral ventricular wall (dashed line) in the 16 and $19 \mathrm{GW}$ human dorsal telencephalon. MOR positive cells were present in the SVZ but did not populate the VZ. Panel C shows that in the E18.5 dorso-lateral VZ of the mouse, MOR staining is present in both the SVZ, and also at the ventricular wall, in contrast to the human foetal staining pattern seen in panels A and B. Scale bars: $50 \mu \mathrm{m}$.

\subsubsection{MOR Co-labelling in the Foetal Human Dorso-Lateral SVZ}

In order to better characterise which types of cell express MOR in the SVZ of the human foetal brain, co-labelling of MOR immunofluorescence was investigated with other markers for glial or neuronal cells. In this study, vimentin was used as a radial glial marker, and nestin was used to determine whether MOR expressing cells were potentially neurogenic. Staining for vimentin (Fig. 4.5) or the other intermediate filament protein nestin, revealed 
the radial glial scaffold which was still present at $19 \mathrm{GW}$. Staining for DCX was used to assess whether migrating neurons or neuroblasts expressed opioid receptors. MOR IR colabelled strongly with vimentin at both 16 and $19 \mathrm{GW}$ (Fig. 4.6, panels A and B), but also co-labelled with nestin (panels C and D). As with the mouse, MOR staining in the SVZ displayed little or no co-labelling with DCX (panels E and F).
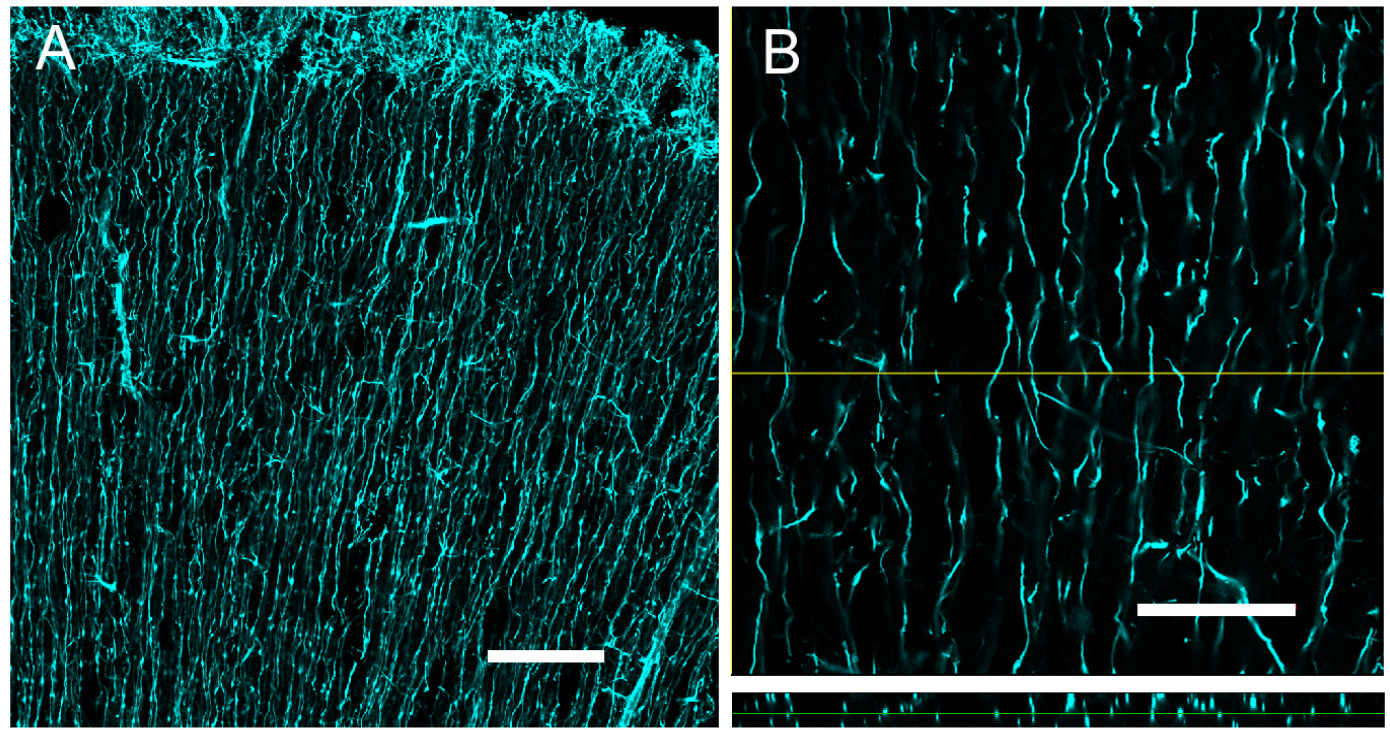

Fig. 4.5. Vimentin staining of the radial glial scaffold at $19 \mathrm{GW}$ in the developing human cortex. Panel A shows a low power view of basal processes of the radial glia in the developing human cerebral cortex stained for vimentin. A higher magnification view (orthogonally cut in the $\mathrm{x}$-axis) shows radial processes in longitudinal as well as in transverse section (shown in the $\mathrm{x}, \mathrm{z}$-axis at the bottom of the picture). Scale bars: $100 \mu \mathrm{m}(\mathrm{A})$ and $50 \mu \mathrm{m}(\mathrm{B})$. 
$16 \mathrm{GW}$
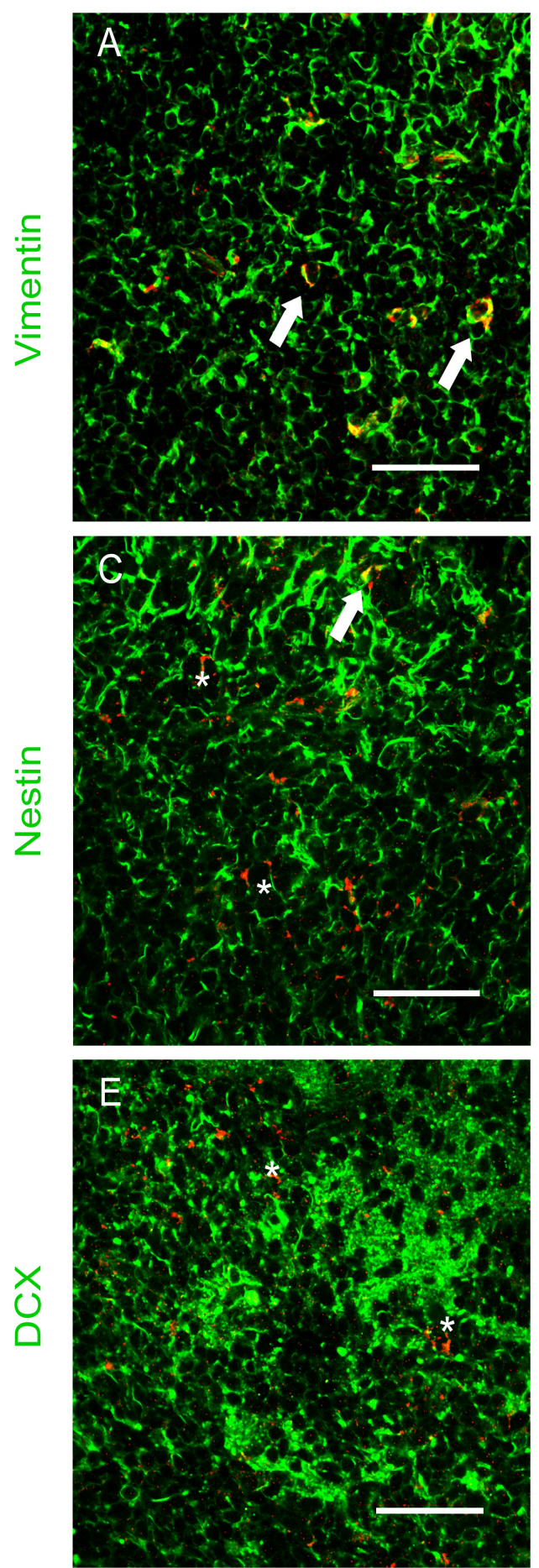

19 GW
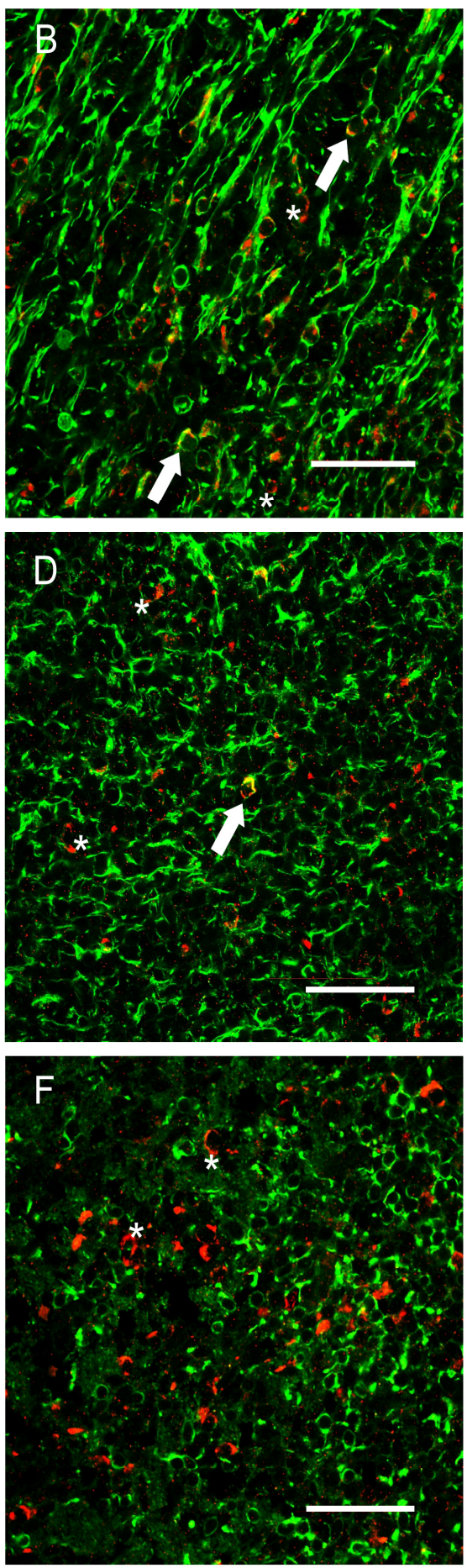
Fig. 4.6. Cellular co-expression of MOR and the markers vimentin, nestin and DCX. MOR IR (red) in the 16 and 19 GW SVZ showed cellular co-labelling with vimentin in a high proportion of MOR IR cells (panels A and B). Co-expression of MOR occurred less with nestin in the two brains examined (panels C and D), whereas, co-expression of MOR and DCX did not occur in the SVZ. Scale bars: $50 \mu \mathrm{m}$.

Co-expression of MOR and other markers was determined by analysing high-resolution images of cells in the $\mathrm{x}, \mathrm{y}$ and $\mathrm{z}$-axes. Examples of cellular co-expression of MOR with the markers vimentin and nestin are shown in Fig. 4.7. As seen in the mouse, cells that expressed MOR in the SVZ showed intense labelling in distinct subcellular locations.

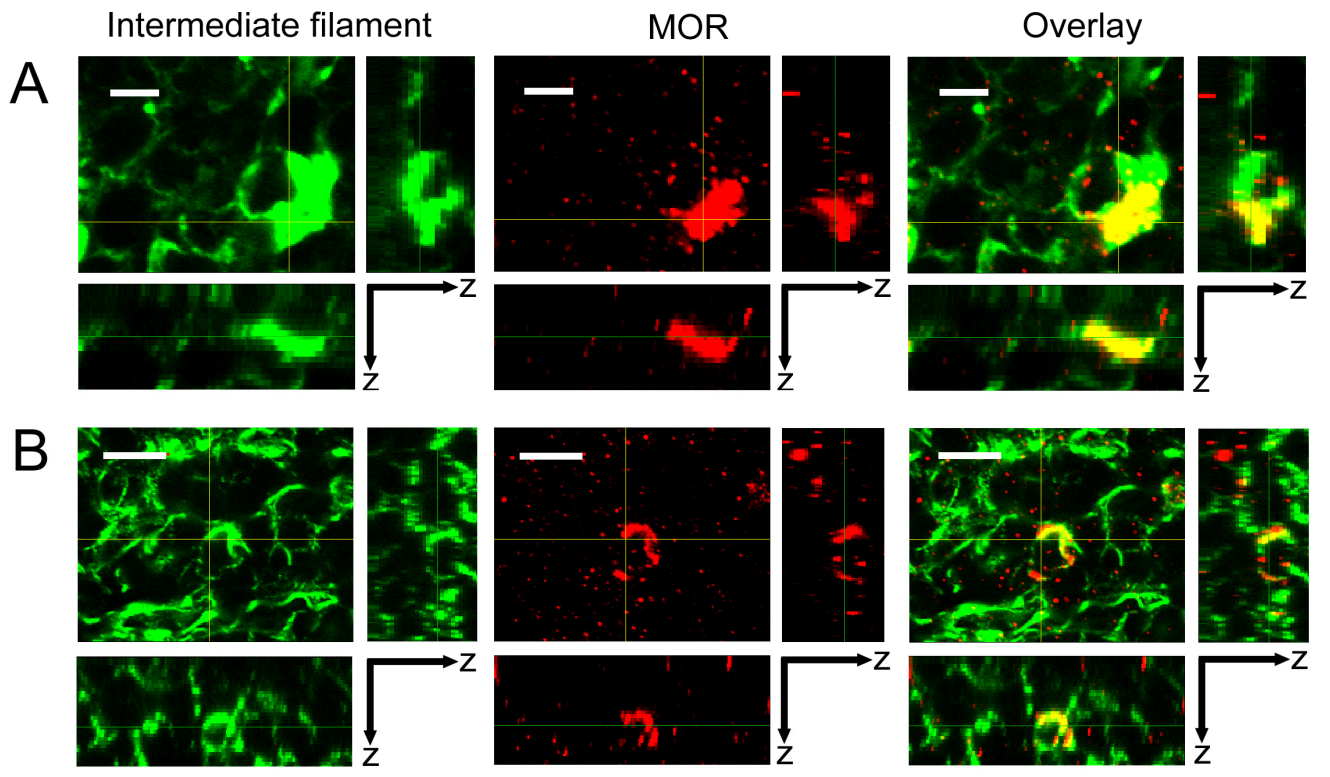

Fig. 4.7. Orthogonal views of cells co-expressing MOR IR and vimentin or nestin. Image series A shows cellular co-expression of vimentin (green) and MOR (red) in a single cell of the 16 GW SVZ. Image series B shows co-expression of MOR and nestin in the $19 \mathrm{GW}$ brain. MOR expression is intense but does not label the entire cell cytoplasm/plasma membrane in a diffuse manner, as seen with vimentin, giving a patchy, localised staining pattern instead. Scale bars: $5 \mu \mathrm{m}(\mathrm{A})$ and $10 \mu \mathrm{m}(\mathrm{B})$. 
In order to characterise cell marker expression of MOR expressing cells, MOR-positive cells from the SVZ were counted, and proportions of cells co-labelled for different markers were determined. Results are presented as a proportion of all MOR expressing cells counted. Cells were counted from a $16 \mathrm{GW}$ and a $19 \mathrm{GW}$ brain (brains 2 and 4). In the 16 GW brain, most MOR-positive cells (86\%) were vimentin-positive. Few MOR-positive cells displayed dual-labelling for nestin (only 9\%), and negligible numbers of cells colabelled for DCX (4\%). In comparison with the $16 \mathrm{GW}$ brain, the $19 \mathrm{GW}$ brain showed that fewer MOR-positive cells co-labelled for vimentin (58\%); whereas, the proportion of duallabelled nestin-positive cells had increased (40\%). No dual-labelled DCX-positive cells could be found.

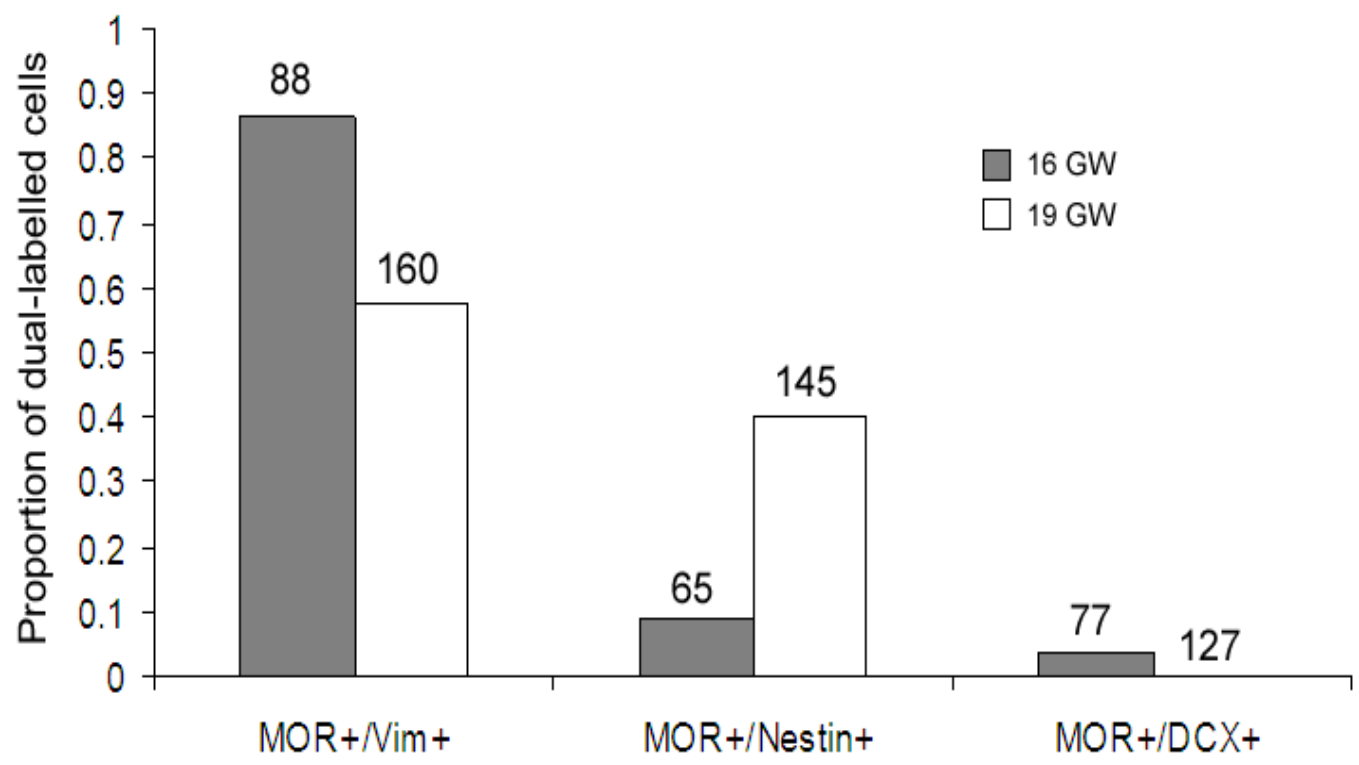

Fig. 4.8. A graph showing proportions of MOR expressing cells that co-express the markers vimentin, nestin and DCX. The majority of MOR expressing cells in the 16 GW SVZ (grey bars) express vimentin, but, less than $10 \%$ of MOR expressing cells also expressed nestin. In the 19 GW SVZ (white bars), proportions of 
MOR expressing cells dual-labelled for vimentin and nestin changed such that fewer cells co-labelled for vimentin, and more cells were co-labelled for nestin. In both brains analysed, very few MOR-DCX duallabelled cells were found. Numbers above the bars represent the number of cells counted for each category.

\subsection{Discussion}

The current chapter has described MOR, as well as KOR, expression in the caudateputamen, where opioid receptor labelling in the human brain has already been characterised (Vonkeman et al., 1996; Voorn et al., 1996; Daunais et al., 2001; Wang et al., 2006). Importantly, MOR staining was also shown in the dorso-lateral SVZ of the $16 \mathrm{GW}$ forebrain. These MOR-positive cells were sparsely populated and stained intensely for MOR IR. Furthermore, they also expressed vimentin, an intermediate filament expressed by radial glia, and some of the cells also expressed the neuronal progenitor marker, nestin. Very few of these cells expressed the neuronal marker, DCX. Preliminary quantitative data showed that the $16 \mathrm{GW}$ brain had higher co-expression of MOR with vimentin; whereas, this double-labelling was much less in the 19 GW brain. Conversely, little MOR-nestin coexpression was seen in the $16 \mathrm{GW}$ brain, but this dual-staining pattern increased four-fold in the $19 \mathrm{GW}$ brain. The MOR-positive cells seen in the human SVZ closely resembled MOR-positive cells of the foetal mouse brain dorsal SVZ. In contrast to the foetal mouse, no marked MOR staining could be identified at the lateral ventricular edge.

The observation that KOR IR was not present in the same regions of human foetal SVZ is consistent with a study by Stiene-Martin et al. (2001) who showed that MOR-IR far 
exceeded that of DOR and KOR in the P2-5 rodent SVZ. However, this result contrasts with the study of Sheng et al. (2007) who showed that cultured nestin-positive neuronal progenitor cells expressed KOR and that stimulation of these receptors promoted cellular proliferation. These neuronal progenitor cells were cultured from 7-9 GW human foetuses, in contrast to the older foetuses used for the present study (16 and 19 weeks old). This difference in developmental stage may account for the lack of KOR IR observed in the present study.

In contrast to the developing E18.5 (Chapter 3) and P2-5 (Stiene-Martin et al., 2001) rodent brains, where MOR IR was found at the ventricular wall, the human brain showed no robust MOR staining at the LV; MOR staining was only present in the SVZ. This difference between rodent and human may be explained by the fact that the rodent and the human VZs are divergent in both relative size and contribution towards the developing cerebral cortex (Dehay and Kennedy, 2007).

Identification of MOR IR glia in the human foetal brain is important because it highlights a similarity to the rodent brain in which glia in the developing late foetal (Chapter 3) and postnatal (Stiene-Martin et al., 2001) animals express opioid receptors and opioid ligands (Vilijn et al., 1988; Hauser et al., 1990; Stiene-Martin et al., 1991a). It also shows that glia within the SVZ of the human may be susceptible to the proliferative effects of direct opioid signalling seen in vitro (Hauser et al., 1996) as well as the proliferative effects observed in vivo (Stiene-Martin et al., 2001). Furthermore, the finding that MOR was expressed on nestin-positive cells shows that some of these cells may have been multipotent neuronal 
progenitors, suggesting that opioid signalling has the potential to directly impact upon neurogenesis in the human foetal forebrain.

As was consistent with the mouse study, MOR expression was restricted to glia (shown by GLAST in the mouse), not neurons or neuroblasts within the proliferative zones. Radial glia at mid-gestation in the human forebrain display marker heterogeneity (Howard et al., 2006). Similarly, the use of two different intermediate filament markers in the present study showed that MOR-positivity was not exclusive to one type of radial glial marker and that one marker could not wholly characterise the type of glial cell that expresses MOR. Two different models could account for this observation. MOR-positive glia could be comprised of a heterogeneous population of cells that have subtly different lineages. Alternatively, MOR expression may occupy one type of cell that is in different states of differentiation such that at any one point in time, MOR expression appears on radial glia bearing different markers. These models are reminiscent of the arguments used to explain marker heterogeneity observed on murine radial glia by others (Malatesta et al., 2003; Anthony et al., 2004, respectively).

Although only two different brains were analysed in detail, each brain at the two time points analysed displayed markedly different co-expression profiles with regards to MOR, vimentin and nestin. It is interesting that MOR-nestin co-expression increased from $16 \mathrm{GW}$ to $19 \mathrm{GW}$, because in the rodent, as time progresses during neurogenesis, nestin expression has been shown to decrease, not increase (Frederiksen and McKay, 1988). MOR has previously been shown to induce nestin expression (differentiation) in embryonic stem cells 
(Kim et al., 2006). However, as only one brain was analysed per time point, it is not possible to determine whether these changes were real shifts in marker profiles of whether the values simply represented experimental variation. Further study with more foetal tissue as well as other glial markers, such as GFAP, to investigate MOR expressing cells within the human SVZ is required. Although, because similar MOR-positive glia that were identified in the SVZ of the rodent were also seen in the human SVZ, the rodent may represent a reasonable model for human brain development, at least in terms of opioid signalling. A large-scale study of MOR-positive glia in the rodent would be of value as a first step in identifying heterogeneity or differentiative dynamics within the population of MOR-positive (thus probably opiate sensitive) glial cells.

In conclusion, the current study has demonstrated with a high degree of certainty that the proliferative, MOR-positive glia observed in the rodent SVZ are also present in the developing human brain. Thus, the developing mouse brain faithfully replicates aspects of these cells in that they are morphologically similar in their staining characteristics and display glial, but not neuronal (DCX), markers. This observation has not been previously reported in the literature and is significant as it demonstrates that opioid system physiology in the developing rodent brain replicates at least some aspects of opioid physiology in the foetal human brain. Given that opioid signalling has been extensively characterised as being modulatory on proliferation and differentiation and that human foetuses are exposed to opiates as a course of analgesic treatment or as a recreational drug in the mother, the characterisation of MOR expression on glia of the developing human brain has significance 
for health of the foetus in mothers exposed to opiates as well as possibly being important as an endogenous modulatory system during normal human brain development. 


\section{Acute Morphine Exposure Lengthens Cell Cycle within the Dorsal Forebrain of the E15.5 Mouse}

\subsection{Introduction}

Several studies have detailed how acute exposure to opioid compounds affects proliferation and cell cycle parameters in the developing forebrain (Vertes et al., 1982; Bartolome et al., 1991; Reznikov et al., 1999; Stiene-Martin et al., 2001). Although these studies outlined the anti-proliferative impact of opiate exposure, they did not extrapolate these effects to the overall development of the brain. For example, Reznikov et al. (1999) showed changes in cell cycle parameters in response to opiates and concluded that opiates modulate proliferation in the developing mouse telencephalon. However, no developmental consequences on brain structure or cognitive/motor function were drawn from these data.

Takahashi et al. (1996a) showed that the developing cerebral cortex can be divided into two cell populations: the $\mathrm{P}$ and the $\mathrm{Q}$ fractions. Early in corticogenesis, radial glia undergo mainly $\mathrm{P} / \mathrm{P}$ divisions to expand the proliferative cell pool. This progenitor pool expansion greatly influences the final cumulative output of neurons since precocious $P / Q$ or $Q / Q$ divisions deplete the progenitor cell pool and cause a dramatic decrease in neuronal output in the final stages of corticogenesis. Whether progenitor cells undergo $\mathrm{P}$ or $\mathrm{Q}$ divisions in the developing cerebral cortex is dependent on cell cycle length, particularly $\mathrm{G}_{1}$ phase 
length (Calegari and Huttner, 2003). Interestingly, INM to the apical VZ also plays a part in maintaining the progenitor cell pool. Altered INM can lead to ectopic mitoses that increase the number of cells exiting the cell cycle (Murciano et al., 2002; Cappello et al., 2006; Tamai et al., 2007). Interference of INM by deletion of Cdc42 results in a massive increase of basal progenitor cells during corticogenesis (Cappello et al., 2006).

Given the known actions of opiates on cellular proliferation, we speculated that morphine exposure during the period of corticogenesis may alter cell cycle progression in dividing radial glia, potentially modulating neuronal output given the putative relationship between cell cycle duration and outcome of radial glial division. Using a time course of BrdU pulse labelling and immunohistochemical detection of mitotic nuclei, we report the effect of acute morphine treatment in the E15.5 mouse embryo on cell cycle progression in the developing cortex.

\subsection{Methods}

\subsubsection{Morphine and BrdU Treatment}

On the morning of E15 (6 am), pregnant dams were injected s.c. with either $10 \mathrm{mg} / \mathrm{kg}$ morphine sulfate (aq) (Hameln Pharmaceuticals GmbH, Germany) or a sham treatment of an equivalent volume of saline. The same dose of morphine or saline was administered every three hours. Six hours after the first injection of morphine, animals were given BrdU 
$(50 \mathrm{mg} / \mathrm{kg})$, intraperitoneally. Animals were sacrificed by cervical dislocation at selected time points.

\subsubsection{Measurement of Proliferation and INM}

To determine whether morphine treatment altered the number of proliferative cells in $\mathrm{S}$ phase, 3 morphine-treated and 3 saline-treated controls were given $\operatorname{BrdU}$, then sacrificed 1 $\mathrm{h}$ later and the pups processed for DAB staining for BrdU incorporation. BrdU-positive nuclei in coronal sections of the dorsal-lateral VZ were counted from the first anterior appearance of the third ventricle in a $200 \mu \mathrm{m}$-wide column, perpendicular to the ventricular surface. Sections were matched to this brain region throughout the study.

To determine whether morphine altered progression of INM, pregnant dams were given BrdU, then sacrificed at hourly intervals over a $6 \mathrm{~h}$ period (Fig. 5.1B). Pups from 9 morphine-treated and 8 saline-treated controls were examined along with pups from 5 morphine-treated MOR KO animals (Schuller et al., 1999) and 6 saline-treated MOR KO controls. MOR KO animals (on a C57BL/6J background) were used to determine whether or not observed changes in INM were due to signalling through MOR and were kindly supplied by Professor John Pintar and Professor MacDonald Christie. To assess INM, the same column of VZ used to determine the number of proliferative cells was stratified into $8 \times 20 \mu \mathrm{m}$ bins from the apical surface of the VZ. BrdU-positive nuclei were considered to reside within a particular bin if they lay within or on the lower-most boundary of that bin. 


\subsubsection{Relative Rate of $G_{2} / M$ transition}

To determine the effect of morphine on $\mathrm{G}_{2} / \mathrm{M}$ progression, 6 morphine-treated dams and 6 saline-treated control animals were sacrificed $2 \mathrm{~h}$ after BrdU injection (Fig. 5.1C). Staining for both BrdU and phospho-histone $\mathrm{H} 3$ was undertaken and the fraction of phospho-histone H3-stained nuclei and BrdU/phospho-histone H3 dual-labelled nuclei determined for the same area of ventricle as for the $1 \mathrm{~h}$ BrdU-labelled animals. At least 200 phospho-histone H3 stained nuclei per animal were counted.

\subsubsection{Morphine Treatment Effect on the Duration of the Cell Cycle and Exit from the}

\section{Proliferative Pool}

To determine whether morphine treatment altered the duration of the cell cycle, 8 morphine-treated dams and 5 saline controls were administered BrdU, sacrificed $16 \mathrm{~h}$ later (Fig. 5.1D), and stained for BrdU and phospho-histone H3. The rationale for the experiment was that after $16 \mathrm{~h}$, a fraction of the BrdU-labelled cohort will have re-entered $\mathrm{S}$ phase and transitioned through $\mathrm{G}_{2} / \mathrm{M}$ such that any dual-labelled BrdU/phospho-histone $\mathrm{H} 3$ nuclei will represent cells that have undergone one complete cell cycle (S phase to S phase). Takahashi et al. (1995b) showed that $16 \mathrm{~h}$ after BrdU injection in the E15 mouse VZ, BrdU-labelled, proliferating cells have transitioned a whole cell cycle and have entered mitosis. Animals received $10 \mathrm{mg} / \mathrm{kg}$ doses of morphine (or an equivalent volume of saline) subcutaneously every $3 \mathrm{~h}$ until $6 \mathrm{~h}$ after the BrdU injection, after which point morphine injections were given every $6 \mathrm{~h}$ until sacrifice. Animals were E15.5 at the time of sacrifice. 
To assess the extent of phospho-histone $\mathrm{H} 3 / \mathrm{BrdU}$ co-labelling $16 \mathrm{~h}$ after BrdU administration, the dorsal telencephalon was divided into medial and lateral halves. Counting in the dorsal telencephalon was further subdivided by categorising cells into apical (VZ) or basal (SVZ) regions. Late $\mathrm{G}_{2}$ nuclei as well as mitotic nuclei were assessed for BrdU incorporation. Nuclei in $\mathrm{G}_{2}$ phase possess punctate, or speckled phospho-histone $\mathrm{H} 3$ (serine 10) staining as a result of progressive phosphorylation of heterochromatin that is required for mitosis (Hendzel et al., 1997). Mitotic nuclei were discriminated from interphase nuclei by intense phospho-histone $\mathrm{H} 3$ staining as well as mitotic morphology. Phospho-histone $\mathrm{H} 3$ (serine 10) staining can be used to discriminate between $\mathrm{G}_{2}$ and mitotic nuclei. Phospho-histone H3 staining is also specific to these phases of the cell cycle as during telophase of mitosis, histone $\mathrm{H} 3$ is dephosphorylated such that no staining can be seen in $\mathrm{G}_{1}$ phase nuclei (Hendzel et al., 1997).

Exit from the proliferative pool (cell cycle exit) was determined by staining for BrdU and Ki67, counting BrdU-labelled cells and determining the fraction that also stained for Ki67. Stained nuclei were counted in a $200 \mu \mathrm{m}$ tissue column comprising the most medial and most lateral regions of the dorsal VZ/SVZ. Approximately 600 BrdU-positive nuclei were counted for each of the medial and lateral regions. The slides were coded such that the researcher counting was unaware whether they originated from a morphine-treated or saline-treated control animal. 


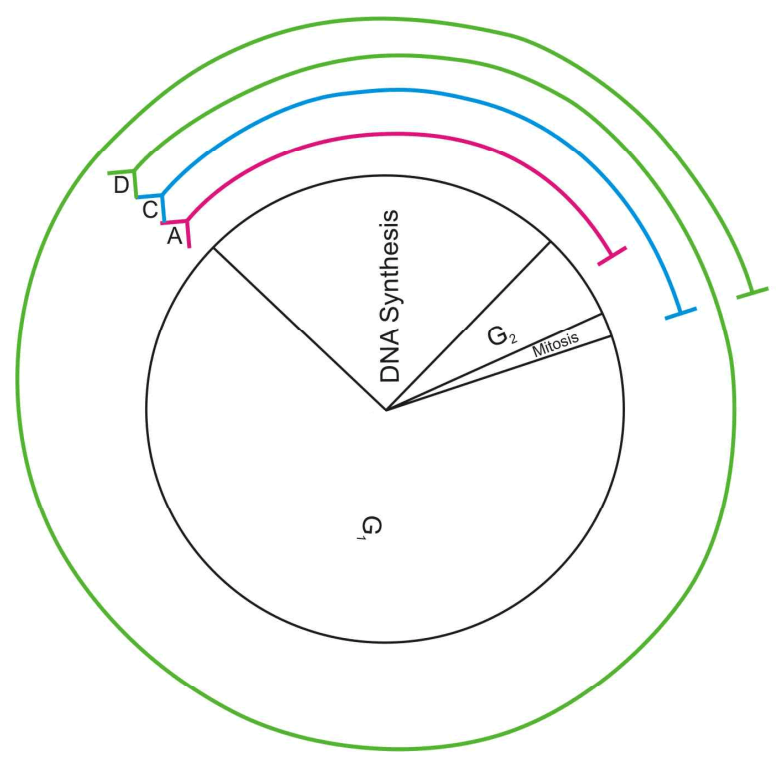

Cell cycle assessment

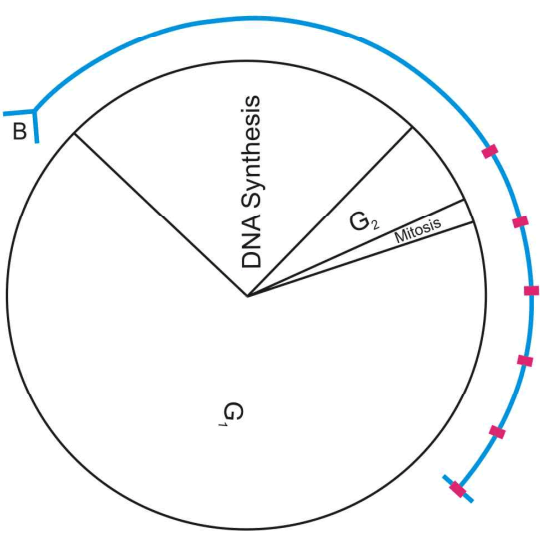

Measurement of INM

Fig. 5.1. Diagrams of BrdU pulse-chase experiments. BrdU pulse-chase paradigms are represented for cell cycle assessment experiments (see 5.2 - Research Design) and for tracking INM of BrdU-labelled nuclei. A) For measurement of the proliferative index of the VZ, animals received an injection of BrdU that labels cells during S phase (DNA synthesis) (red, line A). Animals were sacrificed $1 \mathrm{~h}$ later, approximately half way through $\mathrm{G}_{2}$ phase. B) To follow cohorts of BrdU labelled nuclei in their INM from the basal to the apical VZ as they progress through $\mathrm{G}_{2}$, animals received a pulse of BrdU and were sacrificed at hourly intervals from 1 $\mathrm{h}$ post-injection to $6 \mathrm{~h}$ post-injection (sacrifice represented by red intervals on blue line B). C) Measurement of $\mathrm{G}_{2} / \mathrm{M}$ phase transition required sacrifice $2 \mathrm{~h}$ post-BrdU injection (blue, line $\mathrm{C}$ ) since most mitotic nuclei were labelled with BrdU after $2 \mathrm{~h}$. D) In order to assess cell cycle exit and to make a relative cell cycle measurement, animals received a BrdU injection and were sacrificed $16 \mathrm{~h}$ later (green, line D). At the time of sacrifice, the BrdU pulse-labelled cohort of nuclei that were still proliferative had progressed approximately one full cell cycle. Upon sacrifice, BrdU-labelled nuclei were observed in $\mathrm{G}_{2}$ phase and mitosis, although other BrdU nuclei would also be in $\mathrm{S}$ phase and others would most probably have advanced to $\mathrm{G}_{1}$. 


\subsubsection{Imaging and cell counting}

Brightfield images were captured using an Olympus AX70 photomicroscope (Olympus Optical Co., Hamburg, Germany) equipped with an Olympus DP70 CCD camera. Confocal microscopy was performed using an Olympus FluoView FV1000. Confocal images were overlaid and labelled using Olympus FluoView v1.6a Viewer and Adobe Photoshop CS3 software. Cells were counted using ImageJ software (v1.37).

\subsubsection{Statistical analysis}

Data taken from hourly INM measurements of migrating BrdU cohorts was analysed by an ANCOVA test to allow for time as a co-variate. Data from $\mathrm{G}_{2} / \mathrm{M}$ phase progression, relative cell cycle time and cell cycle exit were assessed for normality using a Q-Q plot and transformed by taking the natural logarithm or the square root of the raw data. Since these experiments involved repeated measurements, analysis of data was performed using the general linear model repeated measures procedure.

\subsection{Results}

\subsubsection{Morphine Slows INM}


Representative images of BrdU-labelled nuclei migrating from the basal VZ to the apical $\mathrm{VZ}$ are shown as a time course for morphine-treated animals and saline-treated controls (Fig. 5.2). One hour after BrdU administration, nuclei from cells in $\mathrm{S}$ phase reside in the basal VZ region; however, by $3 \mathrm{~h}$ post-BrdU treatment, a cohort of labelled nuclei had undergone INM and moved to the apical VZ where they underwent mitotic division. Morphine treatment delayed the INM such that by $3 \mathrm{~h}$ post-BrdU treatment, few labelled nuclei were present at the apical VZ (LV wall) in morphine-treated animals compared with saline-treated controls.

Initial quantitative analysis of INM (Fig. 5.3) showed a difference in the proportion of cells accumulating in the apical VZ between saline-treated and morphine-treated animals. Fig. 5.4, panel A, shows that morphine treatment did not change the number of cells labelled by BrdU (S phase cells); whereas, panel B shows that morphine treatment delayed (or slowed) INM such that it took longer for labelled nuclei to reach the apical VZ $(P=0.013$, ANCOVA) and longer to leave the basal VZ (panel C) ( $\mathrm{P}=0.036$, ANCOVA). This only became evident $4 \mathrm{~h}$ post labelling, a timing effect also observed by Cappello et al. (2006). The basal VZ was defined at a $60 \mu \mathrm{m}$ layer, positioned $40 \mu \mathrm{m}$ basally from the ventricular edge as this region is where the vast majority of nuclei reside during $\mathrm{S}$ phase (Fig. 5.2, $1 \mathrm{~h}$ after BrdU injection). Slower INM did not occur when the experiment was repeated (panel D) using a MOR KO mouse strain. Additionally, no pyknotic nuclei, markers of apoptosis, were observed in any of the groups. 


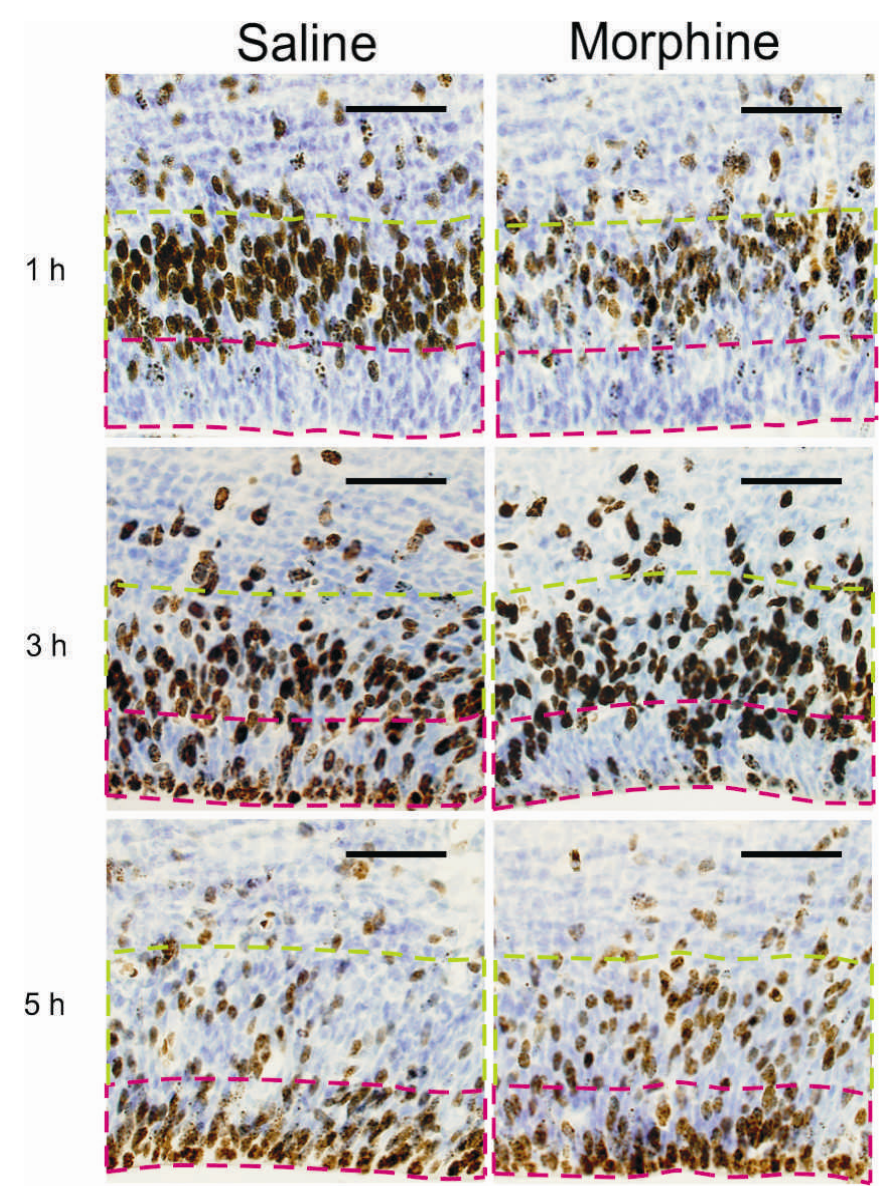

Fig. 5.2. Acute morphine treatment slows INM in the VZ of the LV in the E15.5 mouse. Regions used for cell counting in the basal VZ and apical VZ are indicated by green and red boxes respectively. Nuclei were counted as being in the apical VZ if they resided within $40 \mu \mathrm{m}$ of the lateral ventricle wall, or as basal nuclei if they were in a $60 \mu \mathrm{m}$ region above the apical VZ. One hour after BrdU administration, BrdU staining (brown) is seen in the basal VZ where cells were undergoing $\mathrm{S}$ phase. The saline-treated control animals and morphine-treated animals look similar, however; by $3 \mathrm{~h}$ after BrdU-labelled nuclei have migrated to the apical $\mathrm{VZ}$ during $\mathrm{G}_{2}$ phase, it can be seen that fewer cells accumulated at the apical surface of the VZ in the morphine-treated animals. By $5 \mathrm{~h}$ post-labelling it can be seen that more BrdU-labelled nuclei were left in the basal VZ in the morphine-treated animals. Scale bars: $50 \mu \mathrm{m}$. 
Morphine
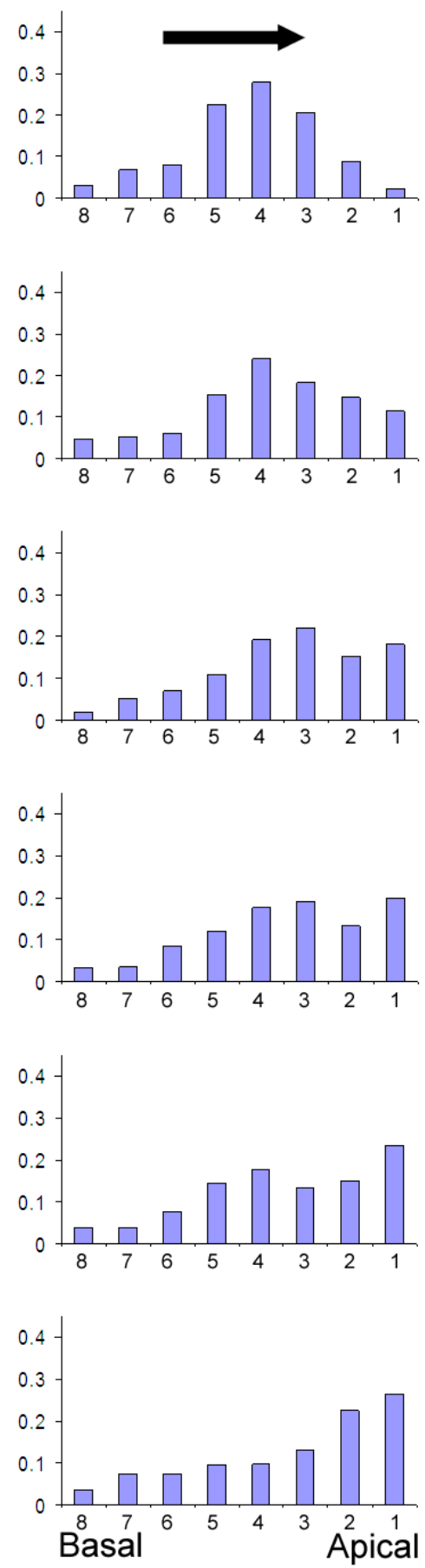

Bin number
Saline

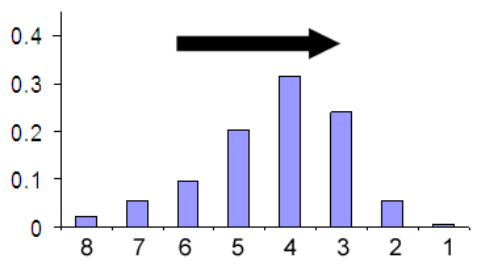

$1 \mathrm{~h}$

Cell cycle

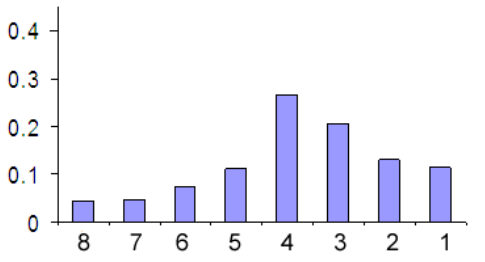

$2 \mathrm{~h}$
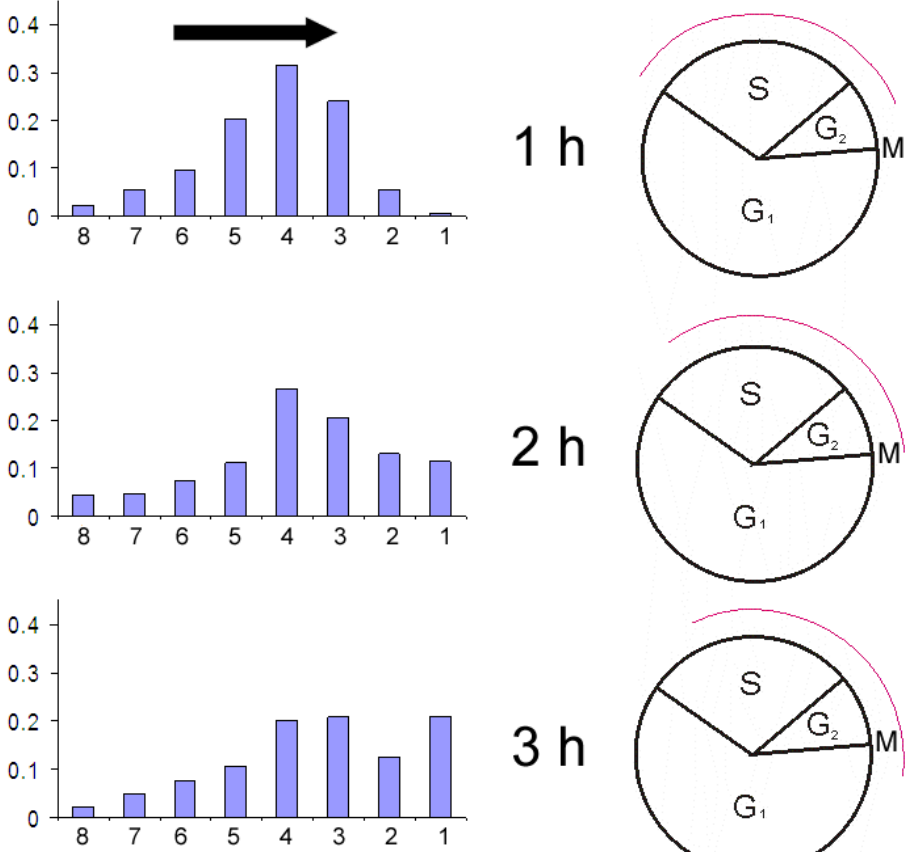

$3 \mathrm{~h}$
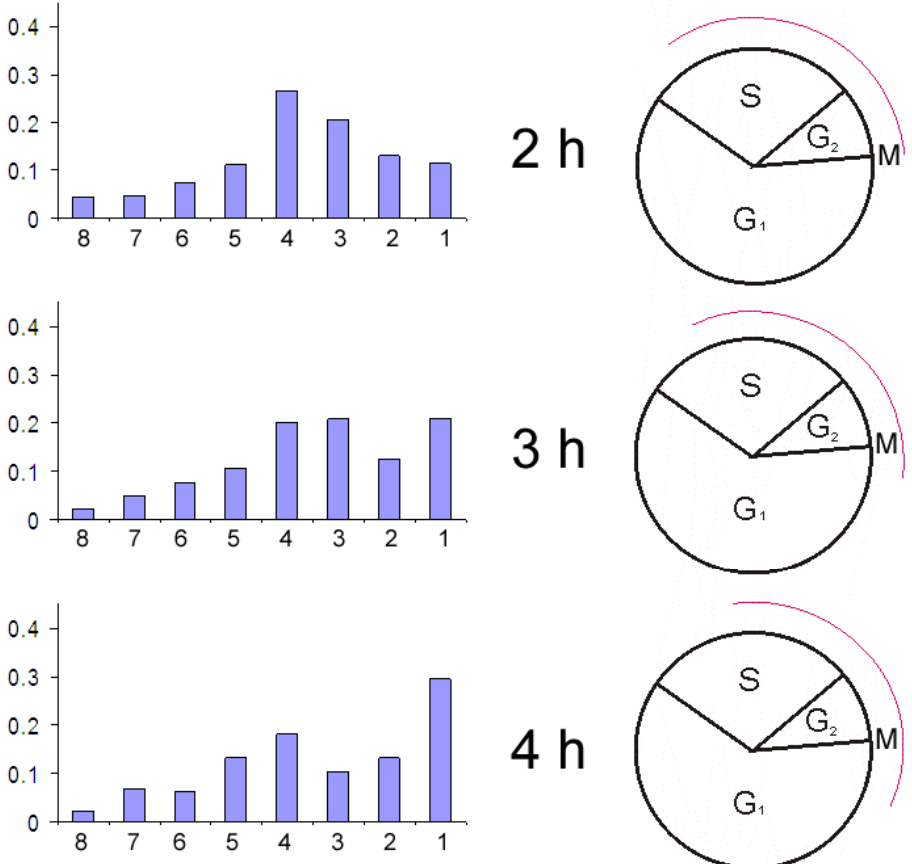

$4 \mathrm{~h}$
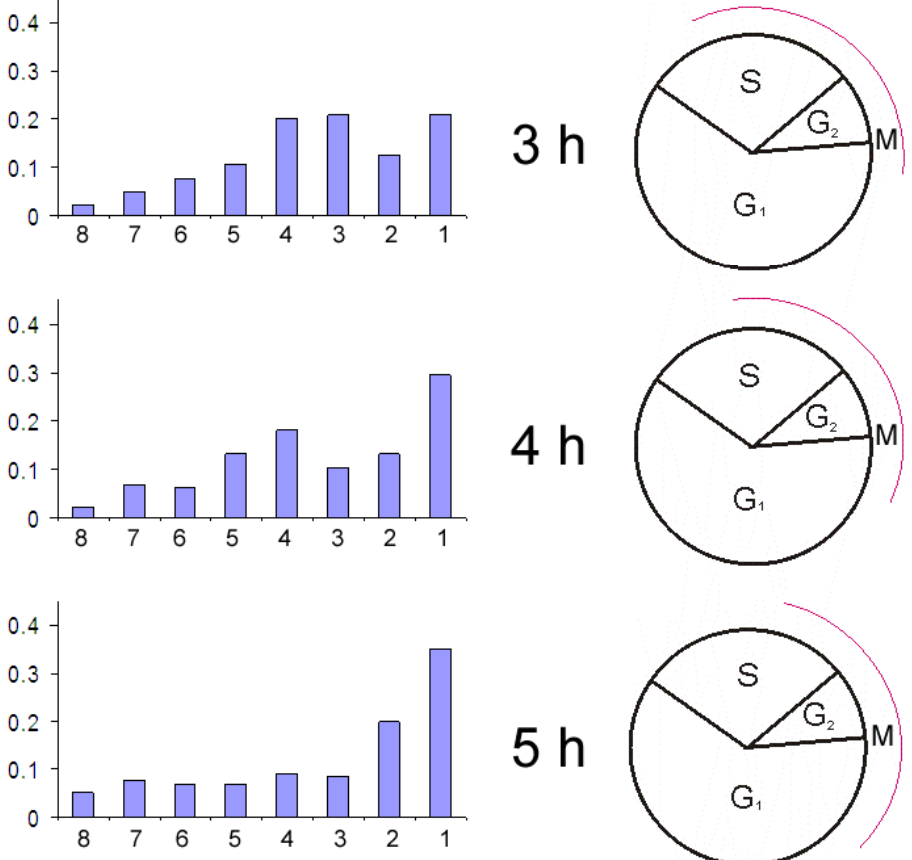

$5 \mathrm{~h}$
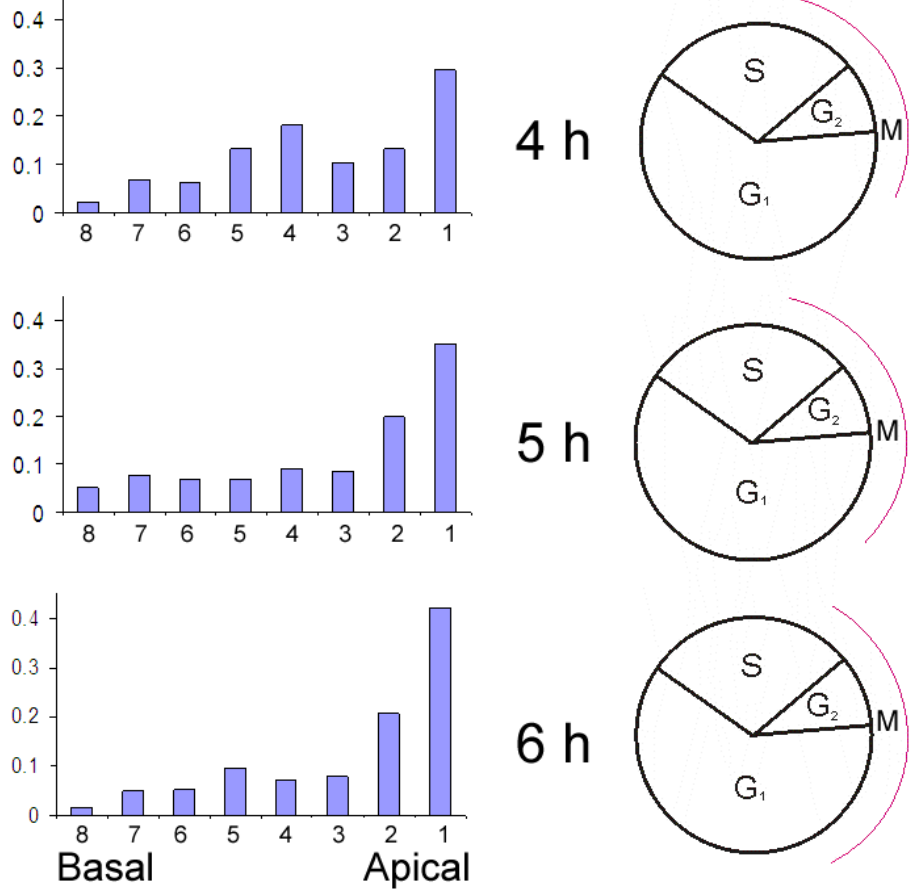

Bin number 
Fig. 5.3. Histogram plots showing INM of BrdU-labelled nuclei within the VZ. Histograms show the proportion of BrdU-labelled nuclei (y-axis) in a given $20 \mu \mathrm{m}$ bin stratified within the VZ (as described in 5.2 - Research Design). Bins represented on the x-axis range from 1 (at the apical surface of the VZ) to 8 (at the basal VZ). As time progresses, BrdU-labelled nuclei occupied more apical positions, as shown by a shift to the right, or the apical side of the histogram (black arrows). Morphine slowed this process down such that BrdU-labelled nuclei took a longer time to accumulate at the apical VZ. Histograms are accompanied by cell cycle diagrams (right) that show which part of the cell cycle BrdU-labelled nuclei progressed to in the salinetreated control animals (red line).
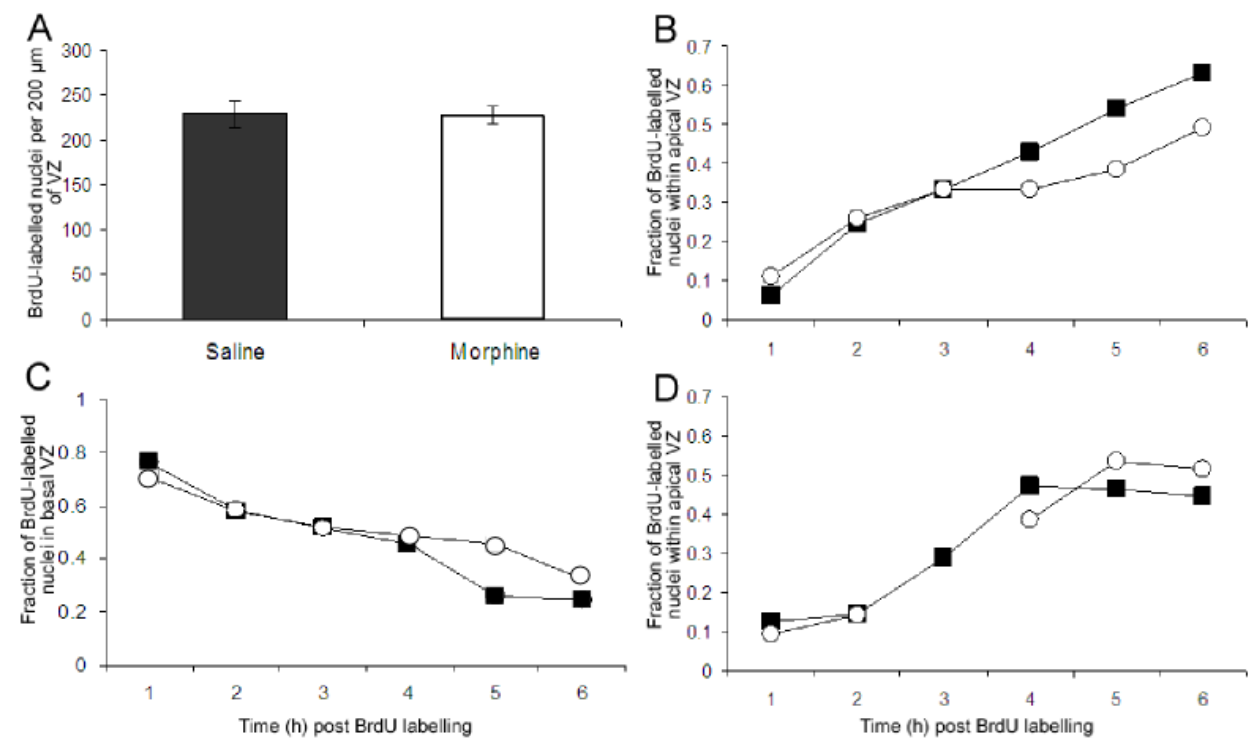

Fig. 5.4. Morphine does not alter total BrdU labelling in E15.5 mouse foetuses but does slow INM in the embryonic VZ. A) Graph A shows that morphine treatment did not change the total number of BrdU-labelled nuclei counted per $200 \mu \mathrm{m}$ length of basal VZ when compared with saline-treated control animals (values are the mean \pm SEM). B) Graph B shows that morphine slowed the migration of BrdU-positive nuclei into the apical VZ, as indicated by accumulation over time of BrdU-labelled nuclei in the most apical $40 \mu \mathrm{m}$ region of the VZ. Morphine-treated animals $(\bigcirc)$ exhibited lower proportions of total BrdU-labelled nuclei in the apicalmost $40 \mu \mathrm{m}$ of VZ compared to saline-treated animals ( $\boldsymbol{\square})$. This effect was only observed $4 \mathrm{~h}$ or more postBrdU labelling. C) Graph C shows that the basal VZ exhibited a complementary pattern of BrdU-labelling over time after the BrdU pulse. The proportion of total BrdU-labelled nuclei retained by the basal $60 \mu \mathrm{m}$ of 
VZ (y-axis, as defined in Fig. 5.3) declined over time; however, morphine-treated animals showed a slower rate of decline, indicating BrdU-labelled nuclei were retained for longer in the basal VZ before migrating to the apical surface. D) Graph D shows that the morphine-induced slowing of accumulation of BrdU-positive nuclei within the apical VZ, observed in C57BL/6J mice (panel B), was not seen in morphine-treated MOR KO mice compared to saline-treated MOR KO mice.

\subsubsection{Relative $G_{2} / M$ Phase Measurement}

To corroborate the finding presented in Figs. 5.2, 5.3 and 5.4, we examined the effect morphine treatment had upon the time required for $\mathrm{G}_{2} / \mathrm{M}$ transition by labelling $\mathrm{S}$ phase cells with a single pulse of BrdU and then $2 \mathrm{~h}$ later sacrificing the animals and determining the number of mitotic profiles that showed BrdU labelling. Cells displaying mitotic profiles were detected by intense staining for phospho-histone H3. Fig. 5.5 shows representative photomicrographs of BrdU-labelled nuclei (green) and phospho-histone H3-stained nuclei (red) $2 \mathrm{~h}$ after BrdU administration. Panel A shows staining from a saline-treated control and panel B from a morphine-treated animal. Cells that were in late S phase had punctate BrdU staining (asterisks) and, as expected, reached the apical VZ ahead of those nuclei that showed solid BrdU staining. In the saline-treated animals, punctate BrdU/phospho-histone H3 staining can be seen at the LV wall in some cells (white arrows), and it is clear that more late S phase labelled-nuclei reached the apical VZ. In the morphine-treated animals (panel B), fewer mitotic profiles (phospho-histone H3-labelled nuclei) co-labelled with BrdU, and less late S phase-labelled nuclei have transitioned to the apical VZ by INM. 
Panel $\mathrm{C}$ shows the fraction of phospho-histone H3-labelled nuclei that also stained for BrdU in the apical and basal VZ for saline-treated controls and morphine-treated dams. For the saline-treated control group, $86 \%$ of the apical phospho-histone H3 stained mitotic profiles also stained for BrdU; while, in the morphine-treated cohort only $43 \%$ were duallabelled. Similarly, $82 \%$ of the phospho-histone H3-positive basal progenitors also stained for BrdU in the saline-treated group while only $41 \%$ were dual-labelled in the morphinetreated group. The effect of morphine on $\mathrm{G}_{2} / \mathrm{M}$ progression within the dorsal telencephalon was highly statistically significant $(\mathrm{P}<0.01)$. 


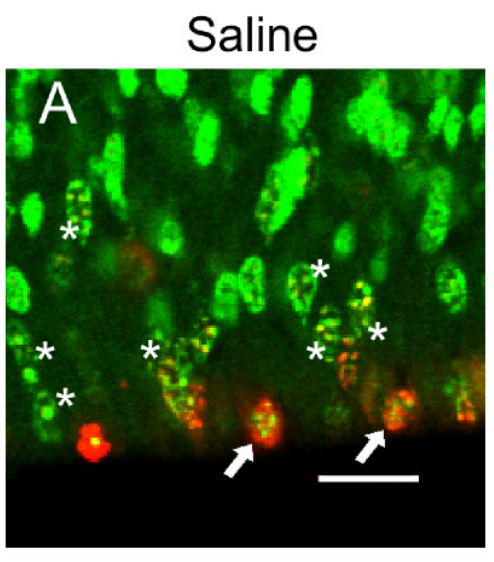

C

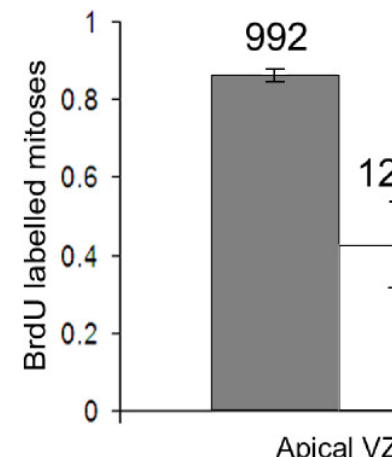

Morphine

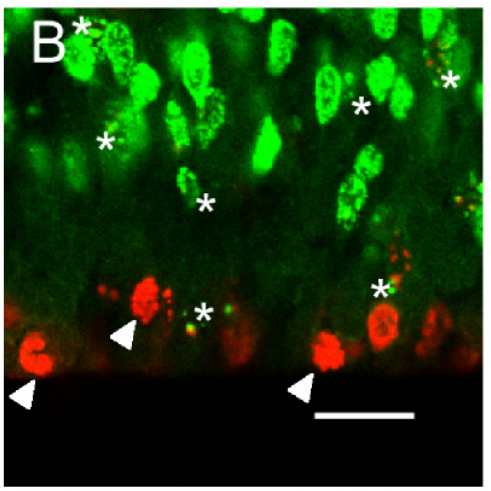

Saline

Morphine

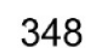 \\ 348}

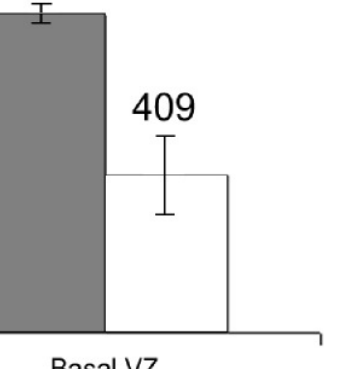

Fig. 5.5. Morphine treatment slows $\mathrm{G}_{2} / \mathrm{M}$ progression in the E15.5 pup. Panels $A$ and $B$ show staining of nuclei for phospho-histone $\mathrm{H} 3$ (red) and BrdU (2 h after BrdU injection) (green) in a representative salinetreated control foetus (panel A) and a morphine-treated foetus (panel B). Many of the phospho-histone H3labelled mitotic nuclei at the apical surface in saline-treated animals showed punctate staining for BrdU (white arrows). Punctate BrdU labelling is characteristic of cells that are in late S phase at the onset of BrdU labelling (asterisks). In the morphine-treated animals, fewer BrdU-positive nuclei displaying punctate labelling were located near the apical surface of the VZ (panel B, asterisks). Similarly, fewer of the apical mitoses in morphine-treated animals stained for BrdU, and thus these nuclei appeared red only (arrowhead). Scale bars: $20 \mu \mathrm{m}$. Panel C graphically depicts the fraction of phospho-histone H3-stained nuclei (mitotic) in the apical and basal VZ that also stained for BrdU. The total number of mitoses counted are indicated above each bar. In the saline-treated controls (grey), most apical and basal mitoses were BrdU-labelled. Acute 
morphine treatment (white) reduced the number of dual-labelled basal and apical mitoses approximately twofold. Values are the mean \pm SEM ( $n=6$ saline-treated control dams and 6 morphine-treated dams).

\subsubsection{Relative Cell Cycle Length}

To determine whether the increased duration of $\mathrm{G}_{2} / \mathrm{M}$ transition caused by morphine exposure also measurably altered the duration of the cell cycle, we examined the fraction of cells that remained proliferative and were able to re-enter $\mathrm{S}$ phase and transition to mitosis for a second time. Preliminary experiments indicated that $16 \mathrm{~h}$ after BrdU administration, sufficient proliferative cells had entered a second round of mitosis to determine if morphine treatment increased the time required to transit the entire cell cycle. Examination of mitotic profiles in conjunction with phospho-histone $\mathrm{H} 3$ staining is able to identify nuclei that are in $\mathrm{G}_{2}$ phase by their morphology and speckled phospho-histone $\mathrm{H} 3$ staining. Mitotic nuclei stained intensely for phospho-histone H3 (red) and appear solid with the image capture settings used to detect $\mathrm{G}_{2}$ phase nuclei (Fig. 5.6). A confocal photomicrograph of the $\mathrm{VZ}$ of a representative saline-treated control animal (panel A) is compared with a morphinetreated animal (panel B). Punctate labelled phospho-histone H3 nuclei (red), indicative of late $G_{2}$ phase nuclei, were seen in transit to the apical surface of the LV (white asterisks). No punctate phospho-histone H3 stained nuclei were detected at the apical VZ. Duallabelling of these late $\mathrm{G}_{2}$ phase nuclei with phospho-histone $\mathrm{H} 3$ and $\mathrm{BrdU}$, administered 16 $h$ previously, identified cell nuclei that were in $\mathrm{G}_{2}$ phase after a second round of DNA synthesis. In saline-treated animals (Fig. 5.6, panel A) several such nuclei could be identified (punctate orange/yellow staining, white arrows); whereas, in the morphine- 
treated animals (Fig. 5.6, panel B) such nuclei were seen in fewer numbers. Orthogonal views in the Z-plane confirmed the phospho-histone $\mathrm{H} 3$ and BrdU staining was located within the same cell nuclei. The location of the dual-labelled nuclei was analysed to determine whether morphine treatment slowed cell cycle progression differently in the medial and lateral regions of the dorsal $\mathrm{VZ}$ or if it affected basal versus apical divisions differently (panels $\mathrm{C}$ and $\mathrm{D}$ ). The fraction of late $\mathrm{G}_{2}$ phase and mitotic nuclei that were BrdU-positive was determined and is shown in panels C and D. 

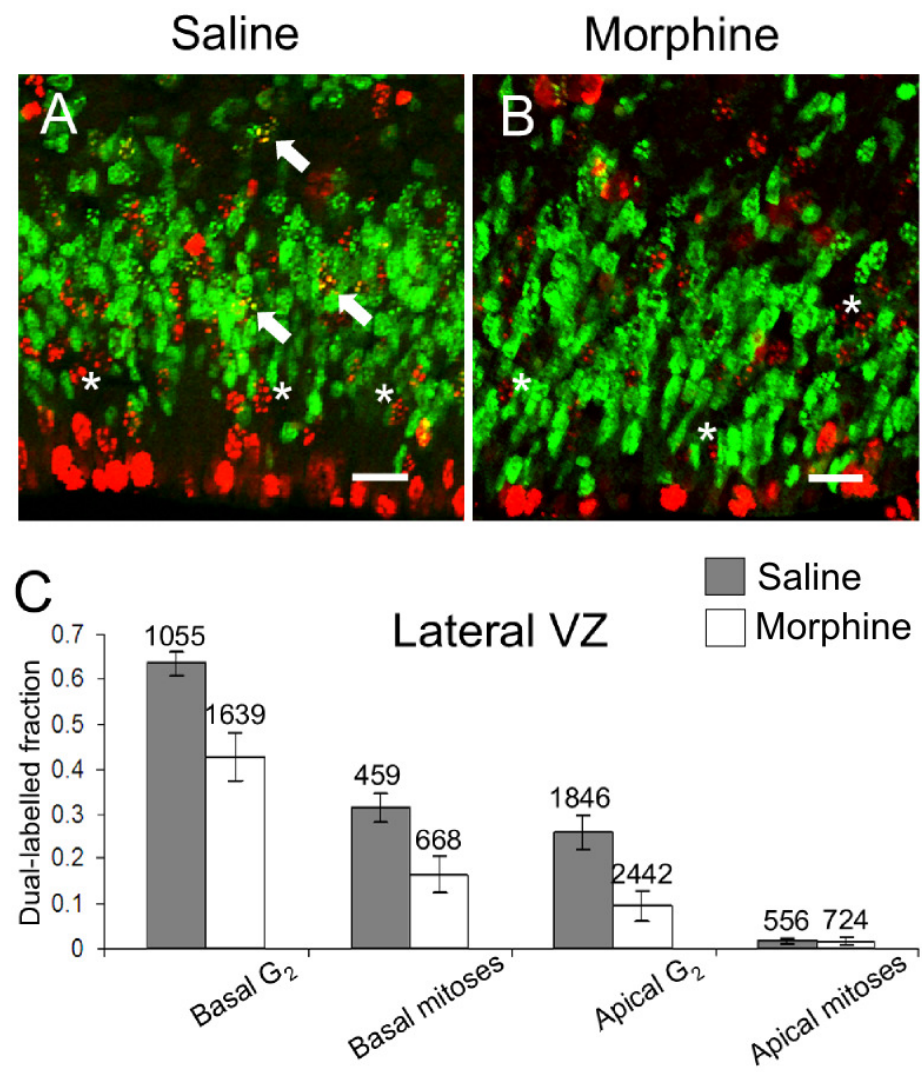

D

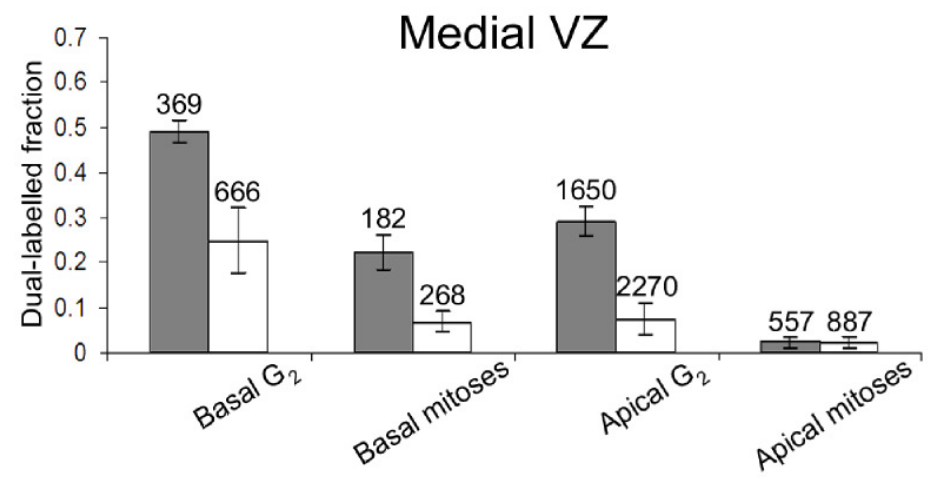

Fig. 5.6. Morphine treatment decreases BrdU/phospho-histone $\mathrm{H} 3$ co-labelling $16 \mathrm{~h}$ after BrdU administration in basal and apical progenitors. Panels A and B show staining for BrdU and phospho-histone $\mathrm{H} 3$, as described in Fig. 5.5, in dams that were administered BrdU $16 \mathrm{~h}$ prior to sacrifice. Of interest are those BrdU-stained nuclei (green) that are undergoing a second round of mitosis and stain for phospho-histone H3 (red). Nuclei displaying punctate phospho-histone $\mathrm{H} 3$ labelling (white asterisks) are in $\mathrm{G}_{2}$ phase (panels $\mathrm{A}$ and $\mathrm{B}$ ). Nuclei dual-labelled for phospho-histone $\mathrm{H} 3$ and BrdU have yellow punctate staining (white arrows) and are readily 
detected in the saline-treated control animals (panel A). Scale bars: $20 \mu \mathrm{m}$. Panels C and D depict the fraction of phospho-histone H3-labelled nuclei that also stain for BrdU in the lateral and medial portions of the VZ, respectively. This population of labelled nuclei was further categorized as either basal or apical in either the medial or lateral portions of the VZ. In addition, the phospho-histone H3 staining was further characterised as to whether the nuclei showed punctate $\left(\mathrm{G}_{2}\right.$ phase) or solid (mitotic) staining. Morphine treatment (white bars) reduced the number of basal BrdU-labelled $\mathrm{G}_{2}$ and mitotic nuclei in both medial and lateral regions. Similarly the fraction of apical BrdU-labelled $\mathrm{G}_{2}$ phase nuclei was reduced in both lateral and medial regions. No significant difference in the number of apical BrdU-labelled mitoses, however, was detected. Values are given as the mean \pm SEM $(n=5$ saline-treated control dams and 8 morphine-treated dams). The value above each bar is the total number of nuclei counted in each category.

In all regions of dorsal telencephalon, including the lateral basal, lateral apical (panel C), medial basal and medial apical (panel D), a higher proportion of $\mathrm{G}_{2}$ nuclei were BrdUlabelled than mitotic nuclei $(\mathrm{P}<0.01)$, supporting our assertion that there is an advancing wave of BrdU-positive cells that are about to undergo mitosis. Interestingly, in both lateral and medial dorsal telencephalon, more basal progenitor $\mathrm{G}_{2}$ and mitotic nuclei were BrdUpositive than the apical radial glia of the $\mathrm{VZ}(\mathrm{P}<0.01)$. The same effect of morphine treatment was seen in both the lateral and medial portions of the VZ. Overall, morphine treatment significantly decreased BrdU/phospho-histone H3 co-labelling in the dorsal telencephalon $(\mathrm{P}<0.01)$. This result was consistent with a morphine-induced slowing of cell cycle. There was little difference, however, in BrdU co-labelling of the apical mitotic cells between morphine-treated and saline-treated controls because of the extremely rare occurrence of BrdU-positive apical mitoses (only 21 and 26 BrdU-positive total apical mitoses out of 1123 and 1611 total apical mitoses were counted in saline-treated animals and morphine-treated animals, respectively). Similarly, morphine treatment ( $8 \mathrm{~h}$ continuous 
morphine exposure, $\mathrm{n}=6$ saline-treated, 6 morphine-treated dams, or $22 \mathrm{~h}$ continuous morphine exposure, $\mathrm{n}=5$ saline-treated, 8 morphine-treated dams) did not alter the relative fraction of basal to apical mitoses (Fig. 5.7) nor did it alter the number of either basal or apical mitoses counted per unit length of VZ. 

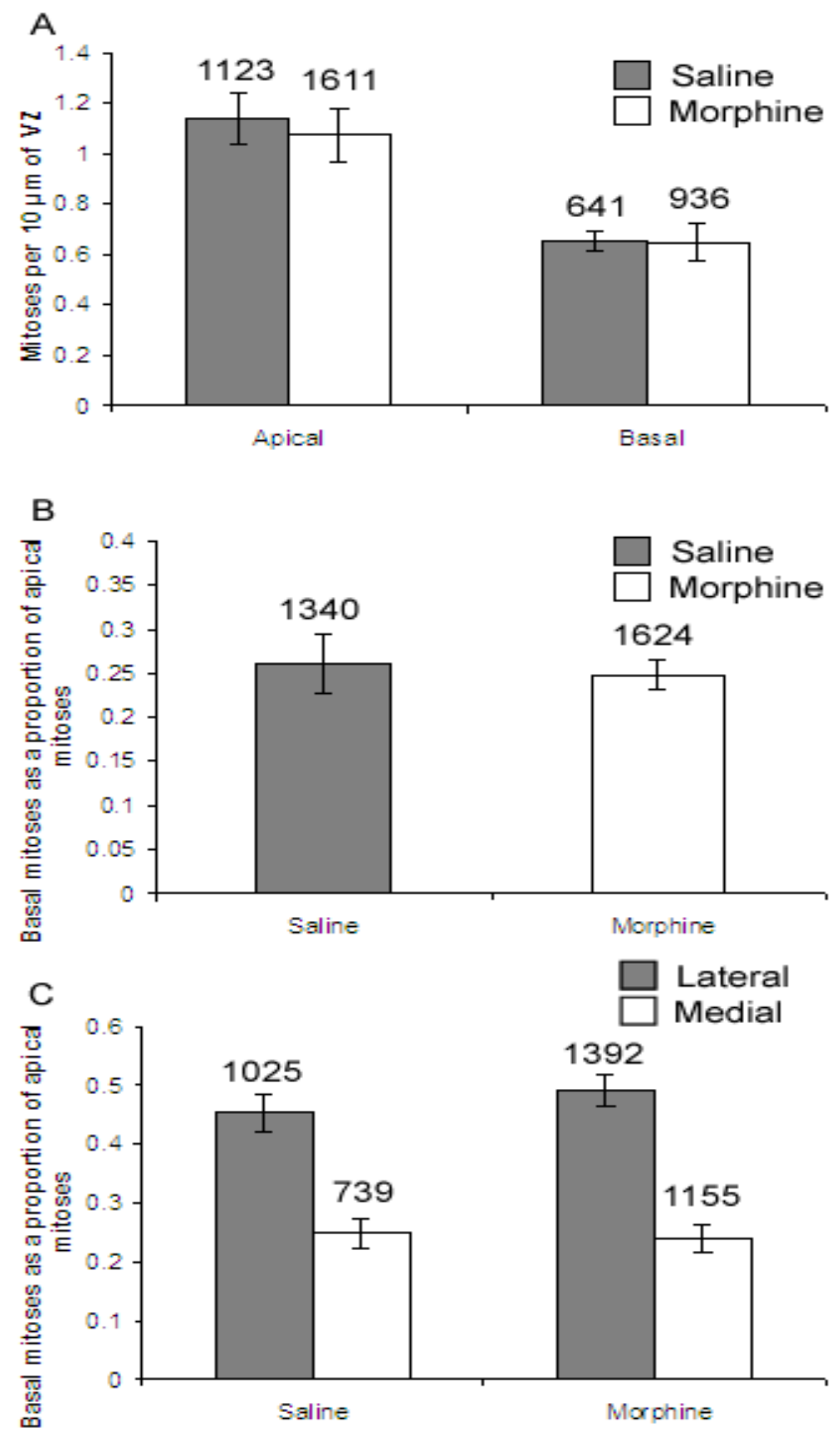

Fig. 5.7. Morphine treatment does not change the number of mitoses or the proportion of basal to apical mitoses. Mitoses detected only with phospho-histone H3 were counted and compared between morphinetreated and saline-treated contol animals in both lateral-medial and apical-basal position. Panel A shows that after $22 \mathrm{~h}$ of morphine exposure, the number of either apical or basal mitoses per unit length of ventricular surface does not change ( $\mathrm{n}=5$ saline-treated litters and 8 morphine-treated litters). Panel B shows that in 
comparison to saline-treated animals (grey bar) morphine did not alter the proportion of apical to basal mitoses after $8 \mathrm{~h}$ of morphine exposure (white bar) ( $\mathrm{n}=6$ saline-treated litters and 6 morphine-treated litters). Similarly, panel $\mathrm{C}$ shows that after $22 \mathrm{~h}$ of morphine exposure, there was no difference in the proportion of basal to apical mitoses. Grey bars represent mitoses in the lateral half of the dorsal VZ; whereas, white bars represent mitoses in the medial half of the dorsal VZ. As expected, in both groups there was a much lower proportion of basal mitoses in the developmentally less advanced medial VZ $(\mathrm{P}<0.01)(\mathrm{n}=5$ saline-treated litters and 8 morphine-treated litters). Values are given as the mean \pm SEM. Numbers above bars represent the total number of mitoses assessed for that category.

\subsubsection{Measurement of Cell Cycle Exit}

To determine whether acute morphine exposure influences whether progenitor cells undergo a proliferative division to yield another radial glial cell and a quiescent neuron (P/Q division) or exit the cell cycle entirely by dividing to form two quiescent cells $(\mathrm{Q} / \mathrm{Q}$ division), the same cohort of animals used to analyze the effect of morphine on cell cycle progression was stained for BrdU and the proliferation marker Ki67. Ki67 is expressed on replicative cells but not on postmitotic cells that have entered $\mathrm{G}_{0}($ Gerdes et al., 1984). Thus, $16 \mathrm{~h}$ after BrdU treatment, nuclei that have stained positively for both BrdU and Ki67 have remained within the cell cycle $(\mathrm{P})$; whereas, nuclei that have stained for BrdU alone have exited the cell cycle (Q). The fraction of BrdU-positive nuclei that stained for Ki67 and the fraction of nuclei that stained for BrdU alone was determined in coronal sections of VZ, divided into medial and lateral portions $(\mathrm{n}=5$ saline-treated controls, 8 morphinetreated animals). Fig. 5.8, panel A shows a representative region of basal VZ that contains nuclei that have stained for BrdU alone (red) (white arrows); whereas, cells that co-stained 
for Ki67 (green) and BrdU appear yellow (asterisks). There was no significant effect of morphine treatment on the number of co-stained BrdU/Ki67-positive cells in either the medial or lateral regions of the $\mathrm{VZ}(\mathrm{P}=0.093)$.
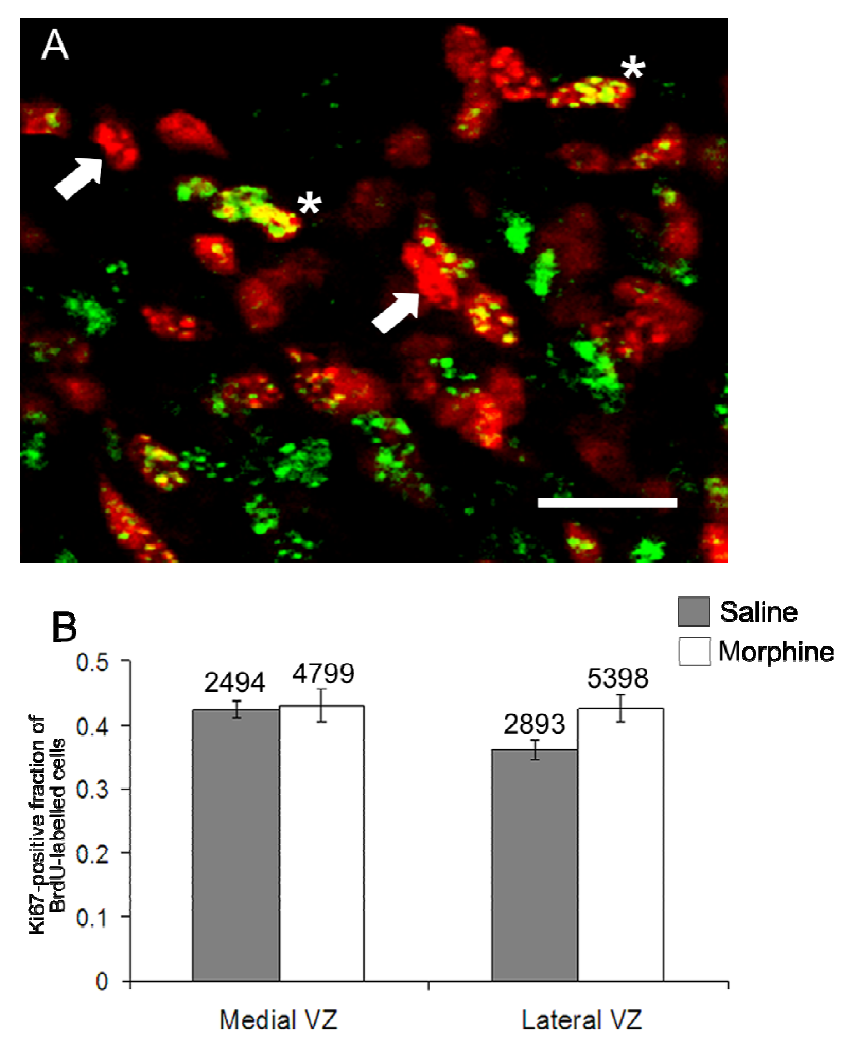

Fig. 5.8. Morphine does not affect cell cycle exit. Staining for the proliferation marker Ki67 (green) and BrdU (red) was performed on the same cohort of animals as in Fig. 5.6. Confocal imaging (panel A) was used to detect labelled nuclei (red) that have exited the cell cycle and therefore do not show green Ki67 IR (white arrows). BrdU-positive nuclei that are still proliferative stain for Ki67 and thus appear yellow (white asterisks). Scale bar: $20 \mu \mathrm{m}$. Panel B shows morphine treatment (white bars) did not alter the fraction of duallabelled Ki67/BrdU-positive nuclei compared to saline-treated control animals (grey bars) in either the medial or the lateral VZ. The fraction of BrdU-positive nuclei that also stained for Ki67 is presented as the mean \pm SEM ( $\mathrm{n}=5$ saline-treated control dams and 8 morphine-treated dams). The values above each bar represent the number of BrdU-stained nuclei counted. 


\subsection{Discussion}

In this chapter, we have shown that activation of opioid receptors by morphine influences cell cycle progression. We have previously reported that radial glia express MOR (Chapter 3), and we provide supporting evidence in the present chapter that morphine causes an increase in the time required for $\mathrm{G}_{2} / \mathrm{M}$ transition. Although morphine acts predominantly through MOR, it can also activate DOR and KOR depending upon the dose used. The effects we observed are, however, likely to be mediated via MOR since slowing of INM to the apical surface of the VZ (Fig. 5.4) was not detected when the experiment was reproduced with a MOR KO strain. Additionally, we were unable to detect DOR on the radial glia in the VZ (Chapter 3, Fig. 3.8, panel B). It is also possible that morphine was acting indirectly on radial glia and basal progenitor cells at a paracrine or even an endocrine level, for example, by modulating the hypothalamic-pituitary-adrenal axis to influence levels of hormones such as growth hormone (Spiegel et al., 1982). However, the expression of MOR on radial glia, in our opinion, favours a direct mechanism of action on the radial glia. Whether or not the morphine mediated effects on cell cycle are consequences of direct opioid signalling through MOR on radial glia, or a downstream effect needs to be the focus of future studies in isolated cells and tissues.

\subsubsection{Technical Considerations}




\subsubsection{Justification of Morphine Dose}

The doses of morphine sulfate given in this experiment were close to what is required for analgesia in the mouse. For example, given s.c., the $\mathrm{ED}_{50}$ for morphine sulfate analgesia in the mouse is approximately $5 \mathrm{mg} / \mathrm{kg}$, as measured by tail-flick latency (Schuller et al., 1999; Gilbert et al., 2004). Mice receiving a morphine hydrochloride dose of $80 \mu \mathrm{mol} / \mathrm{kg}$ (equivalent to $30 \mathrm{mg} / \mathrm{kg}$ morphine sulfate) via s.c. injection reached peak plasma levels of morphine $(18 \mu \mathrm{M}) 25$ min after injection (Handal et al., 2002). The half-life of morphine in mouse plasma was $28 \mathrm{~min}$, and no detectable traces of morphine were found in the plasma $3 \mathrm{~h}$ after s.c. injection. In the same study, no plasma morphine-6-glucuronide was detectable at any time after morphine injection. Based on these results, our dose of 10 $\mathrm{mg} / \mathrm{kg}$ (s.c.) of morphine sulfate would be expected to peak after 25 mins but would probably never exceed a plasma concentration of $6 \mu \mathrm{M}$ (see 8.6 - Appendix 6 for calculations). Morphine would also not accumulate following repeat injections, since injections were given $3 \mathrm{~h}$ apart, more than enough time for morphine to be cleared from the system. However, based on $\mathrm{K}_{\mathrm{i}}$ values of selective opioid receptor subtype ligands and $\mathrm{EC}_{50}$ values using $\left[{ }^{35}\right.$ S $]$ GTPgammaS (a G-protein substrate) (Clark et al., 1989; Mignat et al., 1995; Olianas et al., 2006), these concentrations of morphine are high enough to stimulate all three opioid receptors,. In our study, therefore, as well as being a MOR agonist, morphine would also be stimulating KOR, and to a lesser extent, DOR present in the brain.

\subsubsection{2 $G_{2} / M$ Relative Measurement}


The present chapter used BrdU/phospho-histone $\mathrm{H} 3$ dual-labelling to compare $\mathrm{G}_{2} / \mathrm{M}$ phase length between morphine-treated and saline-treated animals. As a single time-point was chosen ( $2 \mathrm{~h}$ post-BrdU injection) at which to sacrifice animals, a number of technical issues have to be considered in the interpretation of the results.

\subsection{Was $G_{2}$ Phase or Mitosis Lengthened?}

One explanation for the observed decrease in BrdU/phospho-histone $\mathrm{H} 3$ dual-labelling could be that $\mathrm{G}_{2}$ phase was lengthened. However, the same result would have been observed if $\mathrm{G}_{2}$ phase stayed the same length but instead mitosis lengthened. This scenario would have resulted in decreased proportions of mitoses labelled by the $2 \mathrm{~h}$-BrdU pulse and thus a lowered percentage of dual-labelled mitoses. However, as an increased number of mitoses was not observed (Fig. 5.7) and a slowing of INM to the ventricular surface was observed (Fig. 5.4) in the absence of ectopic mitoses, it is very likely that it was $\mathrm{G}_{2}$, and not mitosis, that was lengthened. The existence of 2 different $\mathrm{G}_{2}$ checkpoints has been reported (Xu et al., 2002) that may contribute to the lengthening of the time spent in $\mathrm{G}_{2}$ phase.

\subsubsection{Relative Cell Cycle Length Measurement}

Cell cycle length was compared between saline and morphine-treated animals by labelling cells with BrdU, sacrificing after $16 \mathrm{~h}$ and double-staining for BrdU and phospho-histone H3. This method is analogous to the method used to measure the cell cycle time of basal 
progenitor cells by Takahashi et al. (1995b). Takahashi et al. (1995b) used a time course following BrdU injection to construct a ‘\% labelled mitoses' curve. To conserve animals, we used a single time point $(16 \mathrm{~h})$ in conjunction with analysis of BrdU labelling of two phases of the cell cycle $\left(\mathrm{G}_{2}\right.$ phase and mitosis) using information from phospho-histone $\mathrm{H} 3$ labelling.

\subsection{Was the Observed Difference in Dual-Labelling Due to a Faster or Slower Cell} Cycle Time?

After a survival time of $17 \mathrm{~h}$ post-labelling, Takahashi et al. (1995b) observed 50\% labelling of basal mitoses with $\left[{ }^{3} \mathrm{H}\right]$ thymidine. The present study was reasonably consistent with these results, given that approximately $35 \%$ of mitoses in the basal progenitor cell population had been labelled with BrdU $16 \mathrm{~h}$ post-BrdU injection. The morphine-treated animals displayed much less dual labelling of mitoses. As this analysis only contained a single time-point, it was difficult to determine whether the morphine-treated animals displayed a quicker cell cycle time (more cells had entered and left a second round of mitosis before sacrifice) or a slower one (fewer cells entered a second round of mitosis before sacrifice). However, analysis of $\mathrm{G}_{2}$ phase nuclei BrdU labelling provides a reference point from which one can infer whether cell cycling was faster or slower (Fig. 5.9). If labelling of mitoses in the morphine-treated animal had already peaked and was declining (i.e. a faster cycling), one would expect the $\mathrm{G}_{2}$ phase nuclei to exhibit even less ' $\% \mathrm{BrdU}$ labelling' as they become BrdU-labelled by cycling BrdU-positive cells up to $2 \mathrm{~h}$ (the length of $\mathrm{G}_{2}$ phase in the foetal forebrain (Takahashi et al., 1995b)) before the mitotic 
nuclei. In fact, more $\%$ BrdU labelling of these $\mathrm{G}_{2}$ phase nuclei was observed, indicating that an increased proportion of BrdU labelling of mitotic nuclei immanent. Given that exit from the cell cycle was the same between saline-treated and morphine-treated animals (Fig. 5.4, panel A and Fig. 5.8), and a higher proportion of BrdU staining of $\mathrm{G}_{2}$ phase nuclei than mitotic nuclei indicated an approaching cohort of BrdU-labelled nuclei, a lower proportion of dual-labelling in the morphine-treated animals suggests a slower cell cycle. 


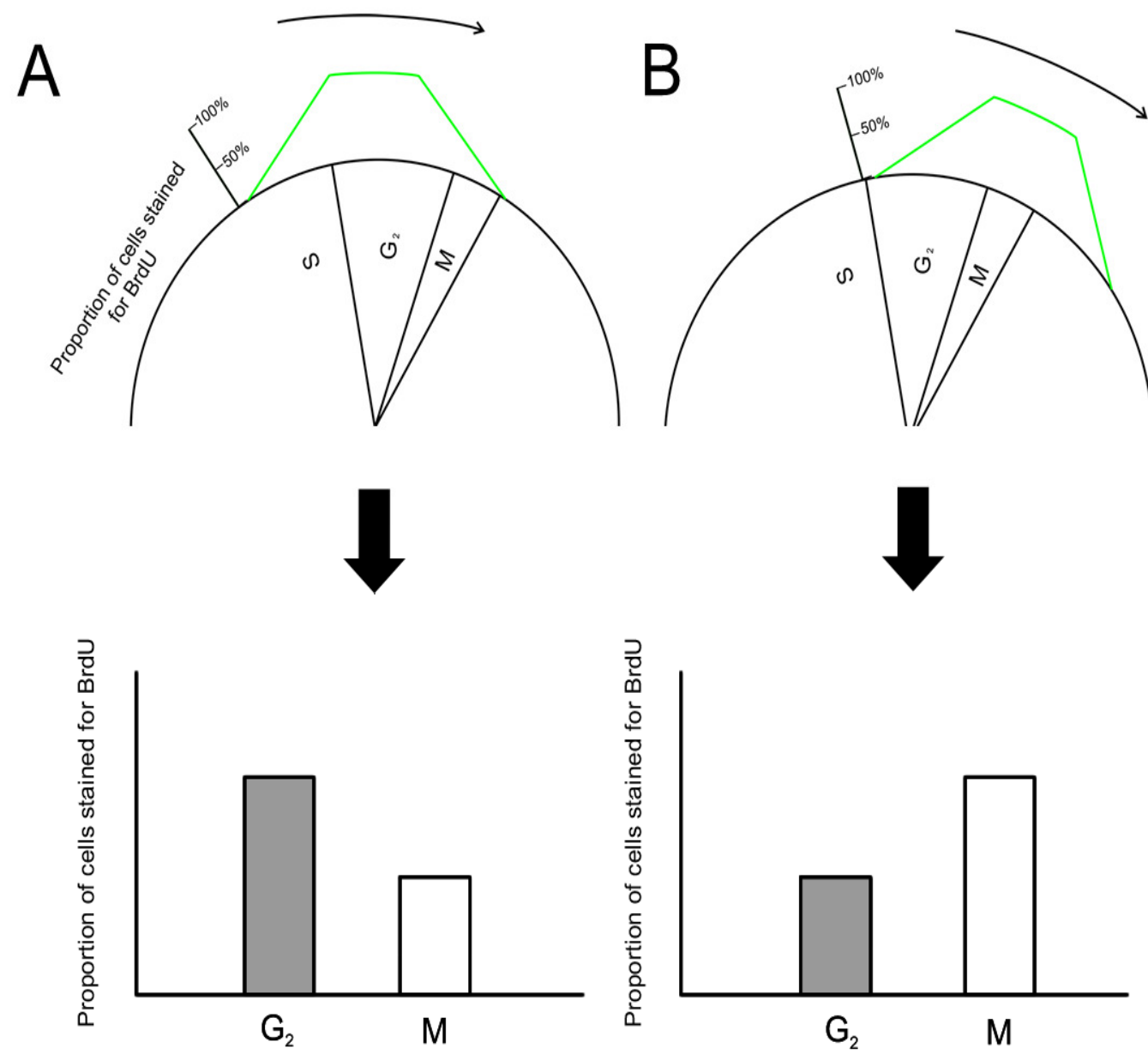

Fig. 5.9. BrdU labelling of $\mathrm{G}_{2}$ phase and mitotic nuclei. Panel A shows a cohort of nuclei approaching mitosis having entered a second round of the cell cycle since BrdU labelling. The green line above the cell cycle diagrams represents the cycling cohort of BrdU-labelled nuclei. Animals injected with BrdU $16 \mathrm{~h}$ prior to sacrifice show a higher proportion of $\mathrm{G}_{2}$ phase nuclei labelled with $\mathrm{BrdU}$ than the proportion of mitotic nuclei labelled with BrdU (refer to the graphs in Fig. 5.6). In contrast, panel B shows that the pattern of a faster travelling cohort of BrdU-labelled nuclei, where most cells have already completed a second round of mitosis. In this case, there is a lower proportion of BrdU-labelled $\mathrm{G}_{2}$ phase nuclei than BrdU-labelled mitoses. As seen in Fig. 5.6, a higher proportion of BrdU-labelled $\mathrm{G}_{2}$ nuclei, as opposed to a higher proportion of BrdUlabelled mitotic nuclei, $16 \mathrm{~h}$ after BrdU labelling, is indicative of an approaching wave of BrdU-labelled cells. 
Therefore, the lower proportions of BrdU-labelled $\mathrm{G}_{2} / \mathrm{M}$ nuclei seen after a second round of the cell cycle could indicate a slower cell cycle in morphine-treated animals.

This experiment also includes an internal control. Takahashi et al. (1995b) showed at E15, that basal progenitor cells had a cell cycle time that was $2.5 \mathrm{~h}$ faster than cells of the VZ. Consistent with this observation, the basal progenitor cells in our experiment showed higher proportions of BrdU labelling than cells in the apical VZ.

\subsection{How Does Cell Cycle Heterogeneity Affect this Measurement?}

Dual-labelling with BrdU and phospho-histone $\mathrm{H} 3$ is insensitive to variations in the cell cycle. It is a snapshot that is biased. The E15 VZ contains a gradient of cell cycle times that range from $17.5 \mathrm{~h}$ in the medial VZ to $21 \mathrm{~h}$ in the dorsal VZ (Miyama et al., 1997).

Calegari et al. (2005) also showed that significant cell cycle time heterogeneity exists within the VZ between neurogenic (Tis21-positive) and non-neurogenic (Tis21-negative) progenitors. The 'snapshot' taken in the current study may only be looking at one population of cells. It is possible that other populations of cells, not detected due to a slower, or much quicker cell cycle time than the dual-labelled mitoses observed, are not affected by morphine.

\subsubsection{Exit from the Cell Cycle - Measurement Using Ki67}


Ki67/BrdU dual labelling is used to measure differences in cell cycle re-entry (Chenn and Walsh, 2002; Hodge et al., 2004), although the biology of Ki67 expression is still the topic of ongoing research. The consensus is that Ki67 labels all cycling cells $\left(G_{1}, S, G_{2}, M\right)$ but not differentiated cells $\left(\mathrm{G}_{0}\right)$.

\subsection{Is Ki67 expression restricted to proliferative cells?}

The absence of Ki67 expression from quiescent cells has been reported in a number of studies (Gerdes et al., 1984; Endl et al., 1997). Although it is generally accepted that Ki67 is only expressed in proliferating cells (Scholzen and Gerdes, 2000), some Ki67 protein expression has been reported in quiescent cells in association with ribosomal RNA synthesis. However, in quiescent cells, Ki67 is greatly down-regulated compared to proliferating cells and requires optimal conditions for visualisation; hence, Ki67 IR cannot be detected in quiescent cells under standard histological conditions (Bullwinkel et al., 2006), as employed in the current study. Thus, it should be a good marker for cell cycle exit in our study.

5.4.1.4.2 How would an increase in cell cycle time impact upon measurement of BrdU/Ki67 dual-labelling?

A slowing in cell cycle would affect which part of the cell cycle a BrdU-labelled nucleus was in at the time of sacrifice. This has implications since if a cell was delayed such that it 
had not yet made the decision to exit the cell cycle and was thus still Ki67-positive, this would artificially increase the observed number of proliferating cells. An increase in proliferating cells was observed in the lateral VZ for the morphine-treated litters (Fig. 5.8, panel B); however, this increase was not statistically significant. Also, the fact that after a 1 $\mathrm{h}$ BrdU pulse no change in the number of BrdU-positive nuclei was observed (Fig. 5.4, panel A) also supports the view that morphine treatment did not significantly alter cell cycle re-entry.

Although the present study found an opiate-induced decrease in the rate of cell cycle progression, it did not corroborate the findings of Reznikov et al. (1999) who reported increases in labelling index $\left(\left[{ }^{3} \mathrm{H}\right]\right.$ thymidine-labelled cells per unit area of VZ) for the MOR and KOR ligands, DAMGO and bremazocine, respectively. We found no such increase in BrdU labelling with morphine (Fig. 5.4, panel A). It must be pointed out that the opiate used in our study is not a purely selective mu opioid ligand, like the synthetic enkephalin, DAMGO. The study by Reznikov et al. (1999), however, must be treated with caution, since a single pregnant female was used for each experimental group. Teratological studies of multiparous species such as mice are confounded by litter effects if the study selects littermates (within a litter) as its base definition of sample size (Haseman and Hogan, 1975; Holson and Pearce, 1992; Zorrilla, 1997).

Modulation of the duration of $\mathrm{G}_{2}$ phase by opiates has been reported in other brain regions and other cellular systems. For example, chronic morphine exposure in the adult hippocampus in vivo markedly decreased cellular proliferation (Eisch et al., 2000) and 
shortened $\mathrm{G}_{2}$ phase (Mandyam et al., 2004). In vitro experiments using the non-selective opiate ligands ethylketocyclazocine and etorphine, which both have low nanomolar affinity for opioid receptors, blocked T47D breast cancer cells in $\mathrm{G}_{2} / \mathrm{M}$ phase (Panagiotou et al., 1999). This opiate-induced cell cycle block occurred simultaneously with an opioid induced modification of the actin/tubulin cytoskeleton. These data are in accordance with our observation that morphine alters INM, a process that is sensitive to microfilament disruption by cytochalasin B (Messier and Auclair, 1974; Murciano et al., 2002). Interestingly, it has also been shown in astrocytes cultured from neonatal mice, which a disproportionately large number of MOR-positive astrocytes were in $\mathrm{G}_{2} / \mathrm{M}$ phase (StieneMartin et al., 1998).

Our observation that both basal and apical mitoses showed a morphine-induced lengthening of $\mathrm{G}_{2} / \mathrm{M}$ phase suggests that morphine is inhibiting the cell cycle of neural/glial progenitors, rather than directly affecting INM. Slowing of INM would occur as well since INM and $\mathrm{G}_{2}$ phase are linked processes in radial glia. The study of Cappello et al. (2006), however, showed that INM in the Cdc42 KO mouse as well as in the Pax6 KO rat (Tamai et al., 2007) was uncoupled with $\mathrm{G}_{2}$ phase duration. In both the studies, disruption of INM caused ectopic mitoses. Murciano et al. (2002) and Messier and Auclair (1974) also demonstrated that pharmacological inhibition of INM with cytochalasin B in other neuroepithelia led to ectopic mitoses, with $\mathrm{G}_{2}$ phase left unchanged. In contrast, Ueno et al. (2006) showed that cell cycle progression is required for normal INM to the apical surface of the VZ. It is of interest that morphine allows the coordination of cell cycle and INM to 
remain intact (i.e. no ectopic mitoses) while other studies have demonstrated that they can be easily uncoupled (Cappello et al., 2006; Tamai et al., 2007).

The VZ of the embryonic brain contains gradients of important extrinsic fate determinants, such as the anti-neurogenic Notch1 (as well as the Notch1 ligand, Delta1) (Murciano et al., 2002). Interestingly, expression of Notch1 is restricted to the ventricular wall, and BrdU labelling has shown that Notch1 is expressed by cells in late $\mathrm{G}_{2}$ phase, but not in cells in $\mathrm{S}$ phase or early $\mathrm{G}_{2}$ (Murciano et al., 2002). Regulation of the duration of $\mathrm{G}_{2}$ phase by opioids could potentially be a mechanism that regulates exposure of cells to ligands that regulate cell fate. While in our study, morphine was not observed to alter cell cycle exit, it cannot be ruled out that morphine may influence developmental maturation and cell fate, as has been shown in cultures of embryonic stem cells (Kim et al., 2006).

In the present chapter, we have shown that morphine exposure in utero has acute effects on progenitor cell behaviour within the $\mathrm{VZ}$ of the developing telencephalon. Acute exposure to morphine slows the arrival of radial glial nuclei to the apical surface of the LV as well as increases the duration of $\mathrm{G}_{2} / \mathrm{M}$ phase of the cell cycle. These effects induced a detectable slowing of the overall cell cycle. Although it is likely that some phases of the cell cycle were left undisturbed, determination of whether a slowing in $\mathrm{G}_{2} / \mathrm{M}$ was the sole contributor to an overall slowing in cell cycle awaits further investigation. The biological implications and the impact on developmental processes of opioidergic perturbation of cortical progenitor cell cycle in vivo warrants future research. 
Results from this chapter have been published in the European Journal of Neuroscience (Sargeant et al., 2008). 


\section{Chronic Morphine Exposure and Histogenesis of the Cerebral Cortex}

\subsection{Introduction}

Studies have shown that opioids inhibit proliferation in astrocytic cells (Stiene-Martin and Hauser, 1990; Stiene-Martin and Hauser, 1991; Stiene-Martin et al., 1991b; Stiene-Martin and Hauser, 1993; Hauser et al., 1996). Given that MOR-IR has been identified on radial glia during corticogenesis (Chapter 3, Fig. 3.7, panel A) and acute morphine exposure affects cell cycle progression (Chapter 5), it is possible that chronic morphine exposure during corticogenesis will affect neuronal output from the VZ/SVZ and impact upon the final neuronal content of the cerebral cortex.

\subsubsection{Chronic Opiate Treatment and Effects on Cellular Proliferation}

In vivo, chronic opioid treatment decreases numbers of neurons within the cerebral cortex. Morphine (10 mg/kg/day) delivered by an osmotic minipump from E12 to P6, decreases neuronal numbers in the P6 rat somatosensory cortex (Seatriz and Hammer, 1993). However, because morphine treatment spanned both corticogenesis as well as a period of postnatal neuronal apoptosis (Verney et al., 2000), it was unclear from this study whether 
morphine treatment acted to reduce cellular proliferation, increase neuronal cell death, or both. The fact that chronic morphine treatment decreases proliferation in the adult hippocampus (Eisch et al., 2000) would favour a mechanism by which morphine decreases proliferation. However, morphine treatment has also been shown to increase fas-mediated apoptosis in the adult brain (Emeterio et al., 2006).

Since opiates can modulate the cell cycle as well as cellular proliferation and since disturbing these processes can have lasting consequences for the tightly co-ordinated sequence of events of corticogenesis, the aim of this study was to determine whether chronic morphine treatment over the period of corticogenesis in the foetal mouse perturbs cortical development. In order to determine whether chronic morphine exposure in utero induced general systemic toxicity, the weights of the pregnant dam, the E18.5 foetus and the P5 pup were analysed. To determine the impact of chronic morphine exposure on the development of the cerebral cortex, neuronal content was assessed both at the end of corticogenesis at E18.5 as well as postnatally, at P5. The P5 pup was analysed as at this stage, cortical laminations become clear and a morphine induced effect had been previously shown in the postnatal cerebral cortex (Seatriz and Hammer, 1993).

\subsection{Research Design}

\subsubsection{Chronic Morphine Administration}


Time-mated pregnant C57BL/6J dams began chronic morphine administration on the evening of E11.5 that lasted until the morning of E18. Morphine sulfate $(10 \mathrm{mg} / \mathrm{kg})$ was injected s.c. into the dams twice daily, once at 9 am and once at 9 pm each at a dose of 10 $\mathrm{mg} / \mathrm{kg}$. Control animals were given equivalent volumes of saline. For analysis of foetal mouse cerebral cortices, pregnant dams were sacrificed by cervical dislocation at E18.5. Mouse foetuses were removed from the uterus and sacrificed by decapitation. Brains were rapidly dissected and fixed overnight in $4 \%$ PFA.

\subsubsection{Morphine Effect on Weight Gain in the Pregnant Dam and Mouse Pup}

Weights of the pregnant dams were recorded from E11.5 to E18, over the period of chronic morphine injection. To determine if morphine had an effect on weight gain in the developing mouse foetus, E18.5 mouse foetuses were dissected from the pregnant dam, as well as from associated amniotic membranes and placenta, blotted on tissue paper to remove mucus and weighed.

To determine if chronic morphine exposure in utero had an effect on postnatal pup growth, pregnant dams were given one last injection of morphine on the evening of E18.5 and were then allowed to give birth on E19. On the morning of birth, approximately three pups from a morphine-treated litter were swapped for the same number of pups from a saline-treated control litter. This cross-fostering was performed to investigate the effect of in utero morphine exposure as well as the effect of drug exposure on the mother on pup weight. 
Pups were allowed to survive until the afternoon of P5, at which point they were weighed and sacrificed by decapitation.

\subsubsection{Effects of Chronic Morphine Treatment on Proliferation and Neuronal Migration in the Foetal Cerebral Cortex}

To assess proliferation in the chronically morphine-treated foetal mouse brain, pregnant mice were injected with BrdU (50 mg/kg) on the morning of E18. Three hours later, pregnant dams were sacrificed, and E18.5 mouse brains were rapidly dissected out from the foetuses and fixed in 4\% PFA. To determine whether or not chronic morphine treatment altered neuronal migration to the outer cerebral cortex, chronically treated pregnant dams were injected with BrdU on E15.5. Pregnant dams were allowed to survive until E18.5, at which point they were sacrificed as described above.

E18.5 mouse brains from both experiments were paraffin embedded and sectioned in the coronal plane at $14 \mu \mathrm{m}$ thickness. To see if chronic morphine treatment had a major impact on proliferation in the E18.5 forebrain or on migration of neurons born on E15.5 to the cerebral cortex, sections were DAB stained for BrdU labelling and background-stained with $0.2 \%$ thionin. 
To analyse DCX and Fas gene expression within the E18.5 chronically morphine-exposed cerebral cortex, brains were rapidly dissected out of the pup, and cortices were microdissected from the brain such that the front $2 \mathrm{~mm}$ of cortex and back $2 \mathrm{~mm}$ of cortex were cut off in a coronal plane and discarded. Cerebral cortex was removed from the remaining brain by removing cortex that lay on top of the striatum. To validate the use of cyclophilin A as an internal control (or 'housekeeping gene'), cyclophilin A mRNA expression was compared to GAPDH mRNA and 18S rRNA expression in chronically treated samples of morphine $(20 \mathrm{mg} / \mathrm{kg} / \mathrm{day})$ or naltrexone-treated $(20 \mathrm{mg} / \mathrm{kg} / \mathrm{day})$ cerebral cortex. Morphine and naltrexone were initially used to analyse the effect of opioidergic modulation on transcription of housekeeping genes. However, experiments using naltrexone were later abandoned to focus resources on reaching conclusive results in the investigation of the effects of morphine on brain development.

\subsubsection{Western Blot Analysis of DCX in the E18.5 Cerebral Cortex}

Western blotting for DCX was performed on the same cortical homogenate samples that were used for real-time PCR analysis. Western blotting for DCX at E18.5 produced two closely associated bands that were semi-quantified by densitometry using ImageJ (v 1.37) software.

6.2.6 Counting DCX Positive Neurons in the E18.5 Cerebral Cortex 
In order to investigate whether a change in DCX expression in the cerebral cortex was due to a change in number of DCX-positive cells, or a change in DCX expression per cell, E18.5 cerebral cortex was fluorescently stained for DCX protein. Coronal sections of cerebral cortex at the level of the first appearance of the $3 \mathrm{~V}$ were immuno-stained for DCX and NeuN. Nuclei were stained with DAPI. NeuN was used to clearly distinguish the cerebral cortex from the intermediate zone and white matter. DAPI-stained nuclei that were positive for DCX staining were counted in $3 \times 50 \mu \mathrm{m}$ columns per section of cortex and three sections of cortex were counted per animal. Measurements of cerebral cortex were performed at the lateral-medial positions of the cortico-striatal junction (lateral) as well as the dorsal-most region of cortex (medial) (Fig. 6.1).
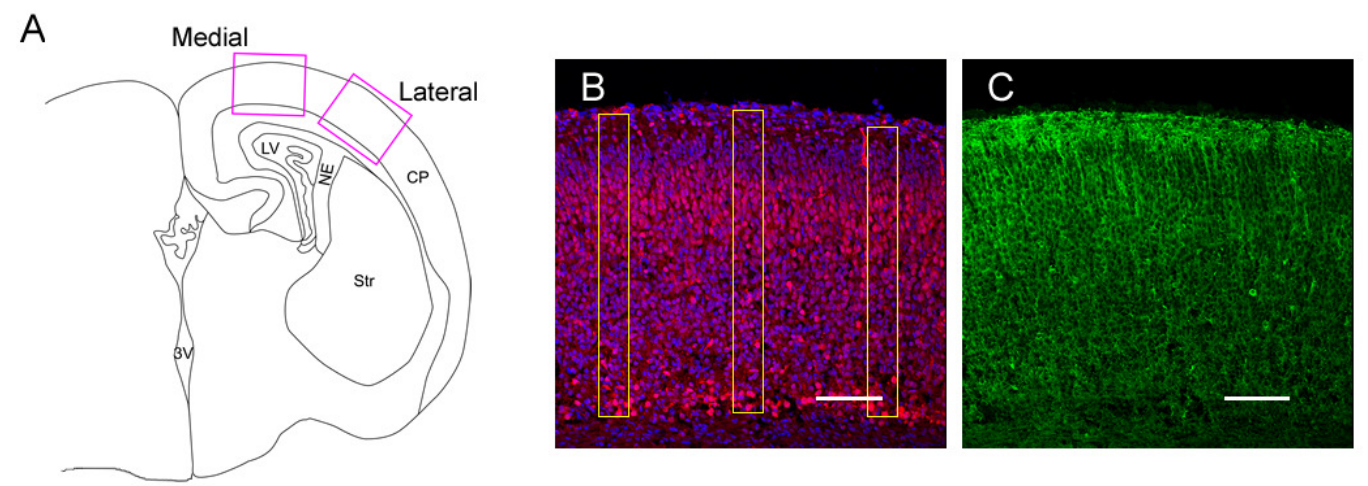

Fig. 6.1. Measurement of the E18.5 cerebral cortex. Panel A shows a trace of an E18.5 brain at the coronal level used for counting DCX-positive nuclei. Pink boxes at medial and lateral positions along the cerebral cortex represent areas where confocal scans were taken. Panel B shows a confocal micrograph of a lateral area of cerebral cortex. Red fluorescence is staining for NeuN; whereas, nuclei are stained blue with DAPI. Yellow rectangles represent the $50 \mu \mathrm{m}$ columns in which DCX-positive nuclei were counted. The counting columns spanned the thickness of the cerebral cortex, as delineated by NeuN staining. Panel C shows the exact same region of cerebral cortex stained for DCX (green immunofluorescence). $3 \mathrm{~V}$, third ventricle; CP, cortical plate; LV, lateral ventricle; NE, neuroepithelium; Str, striatum. Scale bars: $100 \mu \mathrm{m}$. 


\subsubsection{Western Blot Analysis of P5 Cerebral Cortex}

Western blotting was used to determine if chronic morphine treatment induced changes in the abundance of neuronal and glial protein markers present within the P5 mouse cerebral cortex. P5 neocortex was microdissected from morphine and saline-treated mouse brain and homogenised in preparation for western blotting. Cortical homogenates were electrophoresed and immunoblotted for NeuN, GAD65+67 and GFAP. The housekeeping gene GAPDH was used as a loading control.

\subsubsection{Stereological Analysis of Nissl-Stained Neurons in the P5 Neocortex}

Stereological counting was used to determine the number of neuronal nuclei in the cerebral cortex. After paraffin embedding, P5 mouse brains were serially sectioned and Nissl stained with a $0.2 \%$ thionin solution. Random-systematic sampling was performed such that approximately $12 \times 20 \mu \mathrm{m}$ thick sections were sampled per brain, which were a distance of $320 \mu \mathrm{m}$ apart along the rostral-caudal axis. The term random-systematic refers to sampling that begins at a random start point but follows a systematic pattern (for example, every $8^{\text {th }}$ section) thereafter. Ten-twelve coronal sections sampled in a random-systematic manner produces acceptably low levels of variation in stereological estimates of volume and number (Gundersen et al., 1999). 
Stereological analysis was performed on the cerebral cortex, as defined by the Allen Reference Atlas (Allen Institute for Brain Science, 2007; http://www.brain-map.org/). Analysis of the 6-layered cerebral cortex extended medially to include the cingulate cortex and laterally far enough to include cortex up to but not including the entorhinal cortex. Analysis also spanned the entire cerebrum rostro-caudally. Analysis was also broken down into separate categories based on layers. The first two categories consisted of the infragranular layers: layers VI and V. The third category was made up of the supragranular cortical layers: layers IV and II/III.

Neuronal nuclei were identified in thionin-stained tissue according to previously described criteria (Seatriz and Hammer, 1993). Briefly, neuronal nuclei were $5 \mu \mathrm{m}$ or larger and possessed lightly staining nucleoplasm with distinct nucleoli; whereas, glial nuclei were characteristically smaller and were more densely stained. To confirm that these nuclei were in fact neuronal, NeuN immunofluorescence staining was conducted on sections of P5 cortex for comparison (Fig. 6.2). 

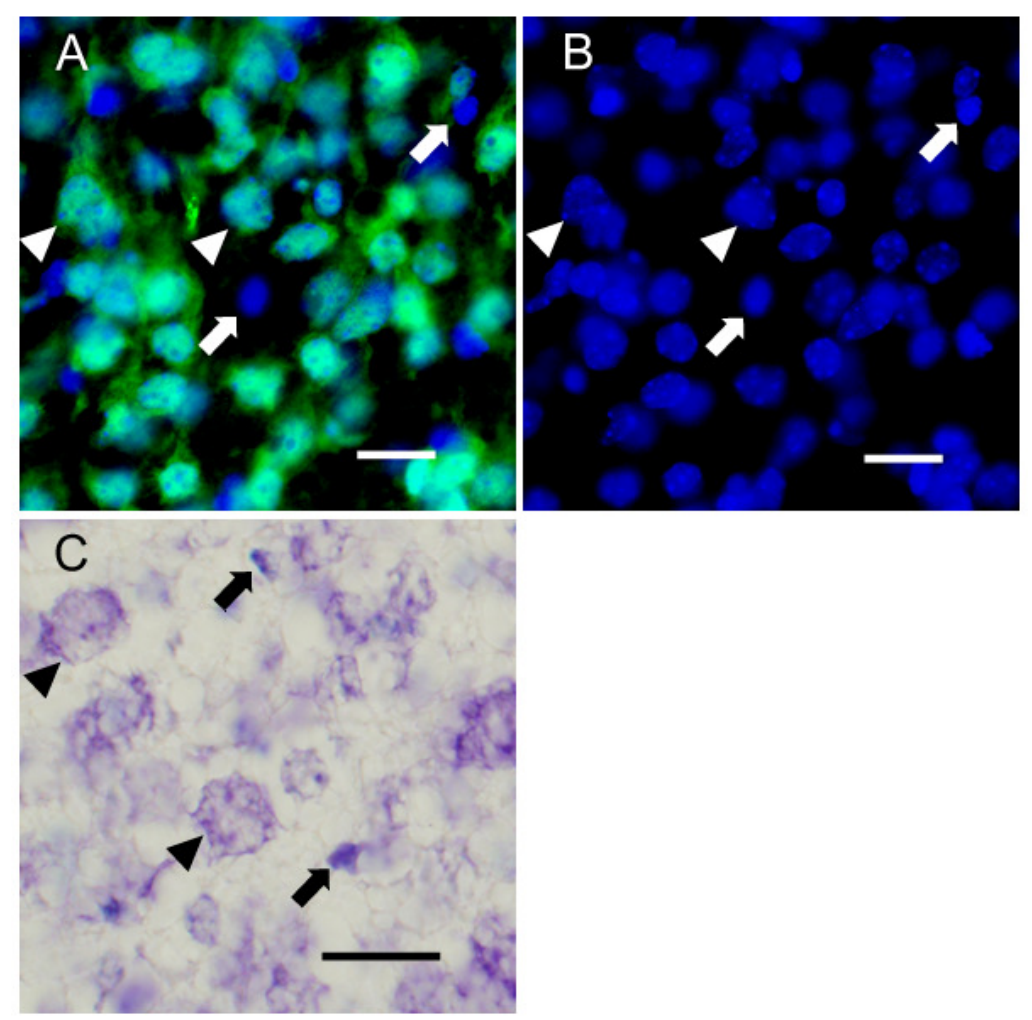

Fig.6.2. Neuronal and glial nuclear morphology. Panel A shows a section of P5 mouse cerebral cortex stained for NeuN (green). Nuclei are stained with DAPI (blue). Arrows show glial nuclei that do not stain for NeuN. Arrowheads show neuronal nuclei that do stain positively for NeuN. The nuclei of the glia that do not stain for NeuN densely stain for DNA (blue) and are smaller than neuronal nuclei (arrows, panel B). Neuronal nuclei are larger and have distinct characteristic multiple nucleoli (arrowheads, panel B). Panel C shows cerebral cortex that has been Nissl-stained with thionin. Arrows show densely staining glial nuclei; whereas, arrowheads show larger neuronal nuclei that have sparsely staining nuclei and contain nucleoli. Some neuronal nuclei are also surrounded by purple Nissl substance (rRNA). Scale bars: $20 \mu \mathrm{m}$.

For estimation of the reference volume (volumes of the cortical layer categories), the Cavalieri principle was used (Gundersen and Jensen, 1987). Put simply, this involves measuring and summating the total area an object occupies on a sampled set of sections. The summated area of this object is then multiplied by the distance that separates any two 
of the sections. For measurement of area, a grid was laid over a video display of a lowmagnification view of the cortical section. The points on the grid were $145 \mu \mathrm{m}$ apart and thus occupied an area of $21,025 \mu \mathrm{m}^{2}$. Points that lay on top of a given category of cortical layer were counted and summated for all 12 serial sections. The reference volume of that layer for that brain was then calculated by the following equation:

$\mathrm{V}_{\text {ref }}=\sum \mathrm{a}_{\mathrm{i}} \cdot \mathrm{t}$

where $V_{\text {ref }}$ is the reference volume, $\sum \mathrm{a}_{\mathrm{i}}$ is the sum of all areas counted for a given object on the 12 serially sectioned slides and $\mathrm{t}$ is the distance that separates the randomsystematically selected sections.

The density of neuronal nuclei within cortical layers was determined using an optical dissector method (see Bonthius et al., 2004). This involved using an unbiased counting frame that was laid over a real-time microscopic view of cortex (Fig. 6.3). Optical dissector samples were taken in a random-systematic raster pattern throughout the object of interest until over 100 samples were taken for the entire sampled set of sections. Sampling 100-200 points is usually sufficient to restrict the coefficient of error due to random noise to 0.1 0.07 (Gundersen et al., 1999). The unbiased counting frame was $20 \mathrm{x} 20 \mu \mathrm{m}$ in its $\mathrm{x}$ and $\mathrm{y}$ dimensions and $8 \mu \mathrm{m}$ in its $\mathrm{z}$ dimension $\left(3200 \mu \mathrm{m}^{3}\right)$. The z-plane movement of the microscope stage was measured by using a linear gage unit (Mitutoyo, Japan). In taking one measurement, the slide was focussed up until nuclei came into sharp focus. The stage was then focussed up a further $2 \mu \mathrm{m}$ in the z-plane to guard against lost objects. Any nucleus in 
focus that lay within the unbiased counting frame at this point was discounted, as this formed the exclusion plane. The cortical section was slowly focussed up by $8 \mu \mathrm{m}$ and neuronal nuclei that came into sharp focus during this time were counted.
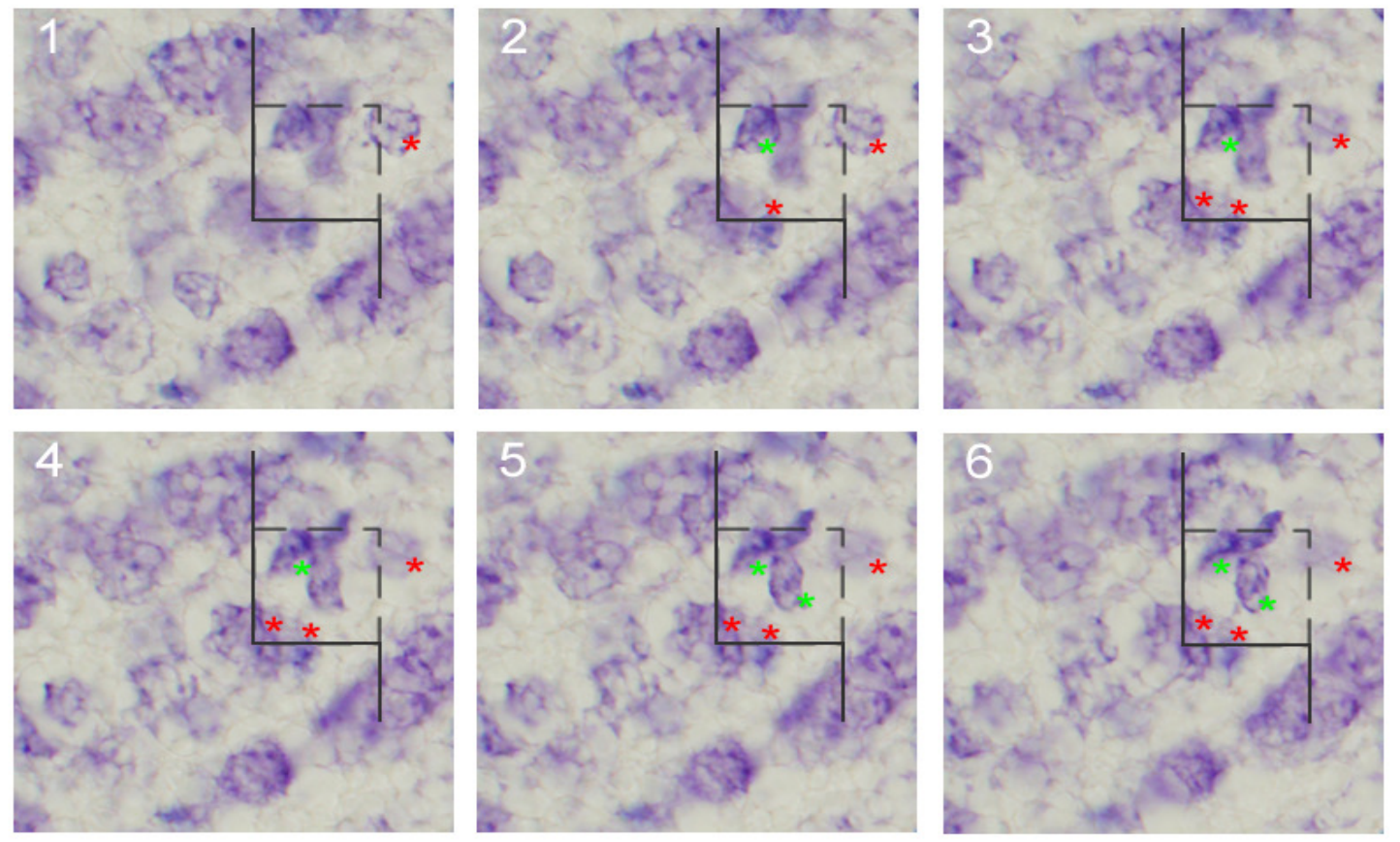

Fig. 6.3. Demonstration of an optical dissector. The unbiased counting frame is represented by the black box, which has an area of 20x20 $\mu \mathrm{m}$. The area within the box is the sample area. The solid left and bottom edges of the box are exclusion planes: if an object touches these lines it is not counted. Conversely, if an object touches the upper or right hand side broken lines it is included in the sample. As an example, these 6 panels represent the same $\mathrm{x}$, y plane that moves $6 \mu \mathrm{m}$ in the $\mathrm{z}$ plane. Panel 1 is the upper exclusion plane. Any nucleus that is in sharp focus on this plane is discounted, represented by the red asterisk. As nuclei come into focus, as in panel 2, they are counted, represented by the green asterisk. In panel 3, two nuclei in the optical dissector come into focus but must be discounted as they touch the solid exclusion lines. In panel 5, one more nucleus of neuronal morphology comes into focus. If this was a complete optical dissector, the end result would be that this optical dissector of 20x20x6 $\mu \mathrm{m}^{3}$ contained 2 neuronal nuclei. 
The neuronal nuclear density of the cortical layers was calculated by dividing the total number of nuclei counted by the total volume sampled by the optical dissector method, and is represented by the following equation:

$\mathrm{N}_{\mathrm{v}}=\sum \mathrm{Q}^{-} / \sum \mathrm{V}_{\mathrm{dis}}$

where $\mathrm{N}_{\mathrm{v}}$ is the density of neuronal nuclei (neuronal nuclei $/ \mathrm{mm}^{3}$ ), $\mathrm{Q}^{-}$represents the number of neuronal nuclei counted per optical dissector and $\mathrm{V}_{\mathrm{dis}}$ is the volume of the dissector.

Using the density and the reference volume, the total number of neuronal nuclei was then estimated by the following equation:

$\mathrm{N}=\mathrm{V}_{\text {ref }} \cdot \mathrm{N}_{\mathrm{v}}$

where $\mathrm{N}$ is the total number of neuronal nuclei.

\subsubsection{Statistical Analysis}

Analysis of variance for the weights of pregnant dams in response to chronic morphine administration, DCX-positive cell counting in the E18.5 cortex and stereological analysis of the P5 cerebral cortex was performed using the GLM repeated measures procedure (SPSS v14). Analysis of pup weights in the cross-fostering experiment was conducted using the GLM univariate procedure (SPSS v14). For determination of the statistical significance of 
the difference between two means when only one parameter was tested, Student's t-test was used.

6.3 Results

\subsubsection{Chronic Morphine Exposure and Weight Gain in Pregnant Dams}

Chronic morphine exposure from E11.5 to E18.5 decreased weight gain in the pregnant dam. At E11.5, pregnant dams weighed an average of 29 g (Fig. 6.4, panel A). However, at the beginning of morphine exposure (E11.5 - E13.5), there is a $1.4 \mathrm{~g}$ weight deficit between morphine-exposed mice and saline controls. Even though weight between the two groups of pregnant dams appears to diverge from E11.5 until E13, normal weight gain is restored after this time. The early reduction in weight gain manifests itself by the end of pregnancy as a small deficit in weight between the two groups (Fig. 6.4, panel A). The difference in weight between the morphine-treated and saline-treated mice is statistically significant $(\mathrm{P}<0.01$, adjustment for multiple comparisons: Bonferroni). Importantly, even though the weights of pregnant dams were decreased by chronic morphine exposure between E11.5 - E18.5, it did not affect the weight of E18.5 embryos (Fig. 6.4, panel B). 
A

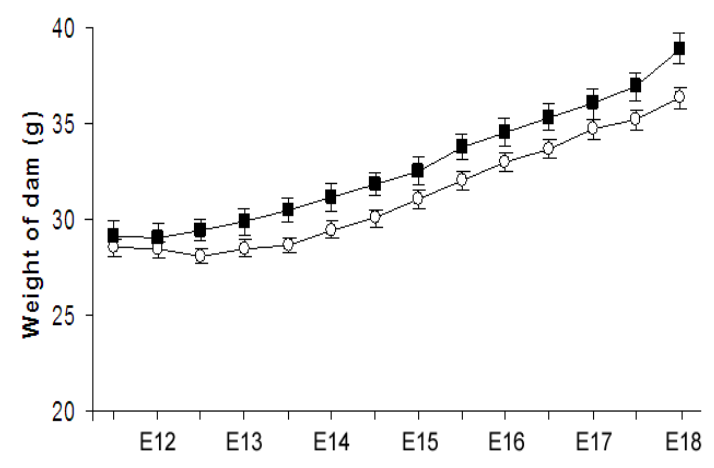

$B$

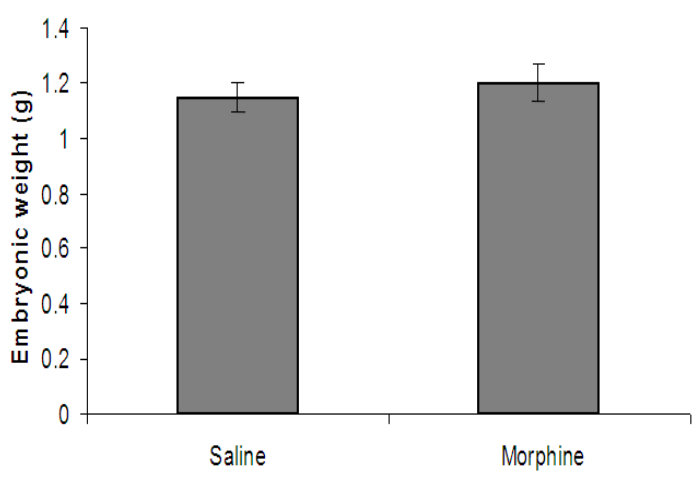

Fig. 6.4. The effects of chronic morphine exposure, in utero, on weight gain in the pregnant dam and in the late mouse foetus. Panel A shows a graph of weight gain of the pregnant dams. Compared to saline-treated controls ( $\mathbf{\square})$, morphine-treated mice (०) display a lag phase from which they recover at E13.5. Panel B shows embryonic weight at E18.5 is not affected by chronic morphine administration.

To investigate whether chronic morphine administration in utero had an effect on postnatal growth of the pup, some pups were cross-fostered to mothers from the opposite treatment group, left to P5 and weighed (Fig. 6.5). Similarly to the E18.5 embryos, chronic morphine treatment from E11.5 to E18.5 on the mouse pups did not alter the weights of P5 mice.

Although in utero exposure to morphine had no effect on weight, morphine exposure on the mother from E11.5 to E18.5 did have an effect on pup weight. Saline-treated and morphinetreated pups raised by a morphine-treated mother were on average $0.32 \mathrm{~g}$ heavier than pups raised by a saline-treated mother $(\mathrm{P}<0.01)$. 


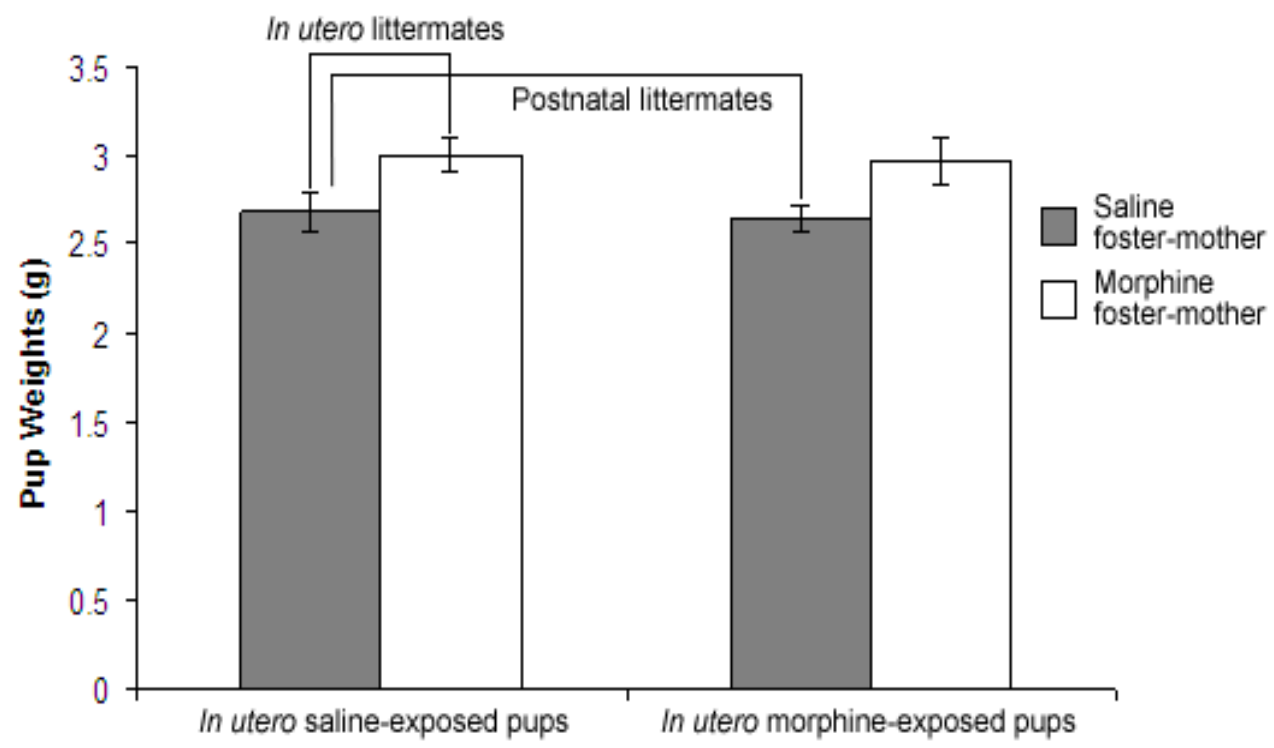

Fig. 6.5. The effects of chronic in utero morphine exposure on the weight of cross-fostered pups at P5. Pups from the same litter at birth either remained with their birth mothers or were re-distributed to mothers from the opposite experimental group and left until P5. At P5, pups that were raised with saline-treated mothers (grey bars) were, on average, smaller than pups raised by morphine-exposed mothers (white bars). However, there was no effect of prenatal drug exposure on the foetus from E11.5 to E18.5 on the weight of the mouse at P5. $n=7$ saline-exposed/saline-mother litters, 9 morphine-exposed/saline-mother litters, 11 salineexposed/morphine-mother litters, 12 morphine-exposed/morphine-mother litters. Values are the mean \pm SEM.

6.3.2 Real-Time PCR Analysis

\subsubsection{Real-Time PCR Validation}

Cortical dissections from E18.5 foetal mice that were chronically exposed to morphine were analysed by real-time PCR. The housekeeping gene that was used in these studies was cyclophilin A. Expression of cyclophilin A was compared to two other housekeeping genes, 
18S rRNA and GAPDH. The expression of cyclophilin A was not found to vary in any consistent direction against these two other housekeeping genes (using the $\Delta \Delta \mathrm{Ct}$ method) when animals that had been exposed to chronic naltrexone $(20 \mathrm{mg} / \mathrm{kg} / \mathrm{day})$ and chronic morphine $(20 \mathrm{mg} / \mathrm{kg} / \mathrm{day})$ were compared to each other. For this analysis, 8 morphine exposed foetuses from 4 litters and 7 naltrexone exposed foetuses from 2 litters were used (Fig. 6.6).

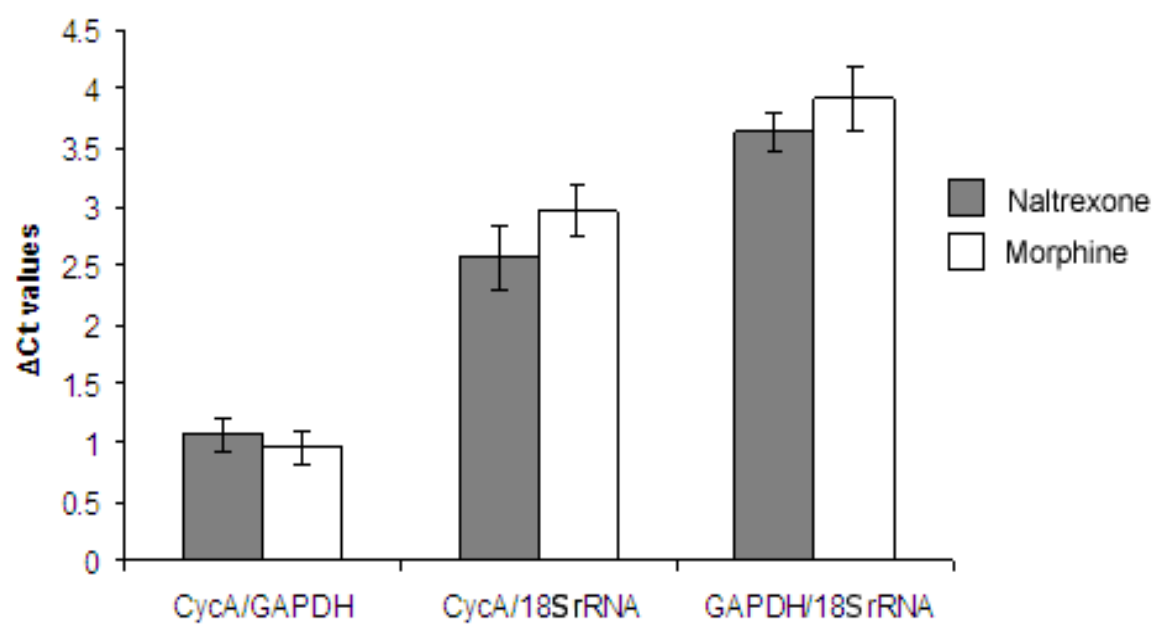

Fig. 6.6. Quantitative comparison of Cyclophilin A with other housekeeping genes. Comparison of cyclophilin A with other housekeeping genes was conducted to determine whether opioid perturbation significantly altered levels of this internal control. Comparison with real-time PCR of GAPDH and 18s rRNA shows that cyclophilin A was not significantly affected when foetuses that were exposed to chronic morphine were compared to foetuses that were exposed to chronic naltrexone, an opiate antagonist. $\mathrm{n}=7$ naltrexone exposed foetuses ( 2 litters) and 8 morphine exposed foetuses (4 litters). Values are the mean \pm SEM (calculated from individual foetuses).

Primer efficiency was calculated for primer sets used in the present study. Template was diluted four-fold ( $2 \Delta \mathrm{Ct}$ values) for each step in a serial dilution that spanned 5 data points 
(Fig. 6.7). All $\Delta \mathrm{Ct}$ values obtained in duplicate were plotted against the log of the fold dilution, and primer efficiencies were calculated with the equation:

Primer efficiency $=\left(10^{(-1 / \text { slope })}\right)-1($ Peters et al., 2004)

All primer efficiencies tested were close to $100 \%$ (Table 6.1) Efficiencies over $90 \%$ are desirable for quantification whereas efficiencies over $100 \%$ indicate the presence of primer dimer (Brisson et al., 2000).

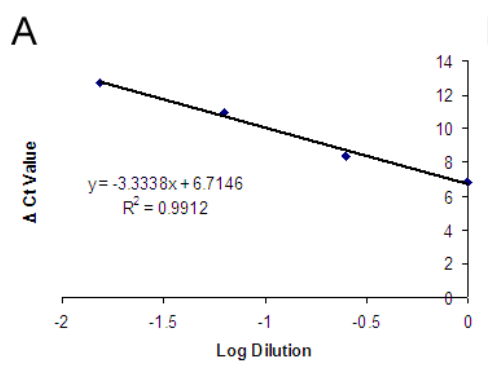

B
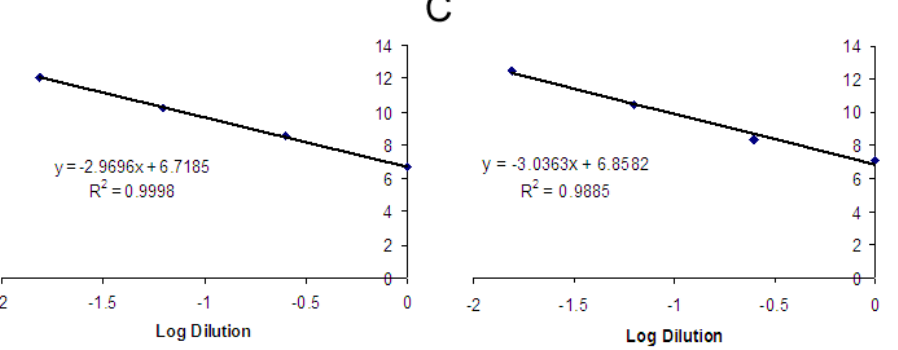

Fig. 6.7. Graphs used to determine primer efficiency values. $\Delta \mathrm{Ct}$ values were plotted against the log of dilution to obtain the gradient that was used to determine the primer efficiency values for primer pairs. Panel A shows values used to calculate the primer efficiency for the primers used to amplify a fragment of cyclophilin A cDNA; panel B shows values obtained for primer pairs used to amplify DCX cDNA sequence and panel $\mathrm{C}$ is for MOR. $\mathrm{R}^{2}$ values shown on the graph demonstrate the quality of the data. Each data point represents the mean of 2 replicates. 


\begin{tabular}{lc}
\hline Primer set & Primer efficiency $(\%)$ \\
\hline & \\
CycA & 99.5 \\
DCX & 117.1 \\
MOR-1 & 113.4 \\
\hline Table 6.1. Primer sets shown with associated primer efficiency values.
\end{tabular}

\subsubsection{Melt Curve Profile Analysis}

To further assess the suitability of the selected primer sets, melt curve profile analysis was performed that allowed analysis of the purity of the respective PCR products. Melt curve analysis involves measuring the fall of fluorescence signal from Sybr green I intercalated with double-stranded DNA plotted against rising temperature of the reaction mixture. As a specific sequence of DNA will have a characteristic temperature at which the double helix will melt apart (which will reduce Sybr Green I fluorescence), the presence of a specific PCR product will provide a sharp peak on a graph that plots the first derivative of relative fluorescence units over temperature versus temperature of the reaction mixture (dRFU/dT vs. T). This represents the rate of change of fluorescence plotted against reaction temperature. A single peak on such a graph indicates the presence of a single product whereas a low diffuse peak from $75-85^{\circ} \mathrm{C}$ is indicative of the presence of primer dimer.

The melt curves for cyclophilin A, MOR and DCX PCR products all produce a single peak at $87^{\circ} \mathrm{C}, 90^{\circ} \mathrm{C}$ and $85^{\circ} \mathrm{C}$, respectively (Fig. 6.8, panel C). Melt curve analysis of negativecontrol PCR reactions was also carried out to determine if contamination could have 
confounded the experiments. Both reactions that used $\mathrm{H}_{2} \mathrm{O}$ as well as equimolar untranscribed mRNA as template did not display any fluorescence specific for the PCR products (Fig. 6.8, panel D). As well as ruling out contaminating PCR product, this also demonstrated that there was no measurable contaminating genomic DNA in the PCR reactions.

\subsubsection{Expression of MOR within the E18.5 Dorsal Telencephalon}

Data obtained from these experiments showed that expression of MOR mRNA was not significantly altered by chronic morphine administration compared with saline-treated controls. These results indicated that MOR mRNA is expressed within the dorsal cerebrum of the E18.5 foetus, corroborating the results of our IHC on MOR protein expression in this region (Chapters 3 and 4). MOR transcript was approximately $4000\left(2^{12}\right)$ fold less abundant than the ubiquitous cyclophilin A transcript or $500\left(2^{9}\right)$ fold less expressed than DCX mRNA within the dissected volume of tissue.

\subsubsection{Chronic Morphine Exposure and DCX mRNA Expression in the E18.5 Cerebral}

\section{Cortex}

Chronic morphine exposure over the period of corticogenesis significantly decreased DCX mRNA expression within the E18.5 neocortex $(\mathrm{P}=0.016$, Student's t-test, $\mathrm{n}=8$ salinetreated dams, 8 morphine-treated dams). Morphine-treated animals showed an average 
increase of $0.35 \Delta \mathrm{Ct}$ units compared with saline-treated animals (Fig. 6.8, panel A). This corresponds to a $22 \%$ decrease $\left(1 / 2^{\Delta \mathrm{Ct}}\right)$ in DCX transcript expression in chronically morphine-treated cerebral cortices.

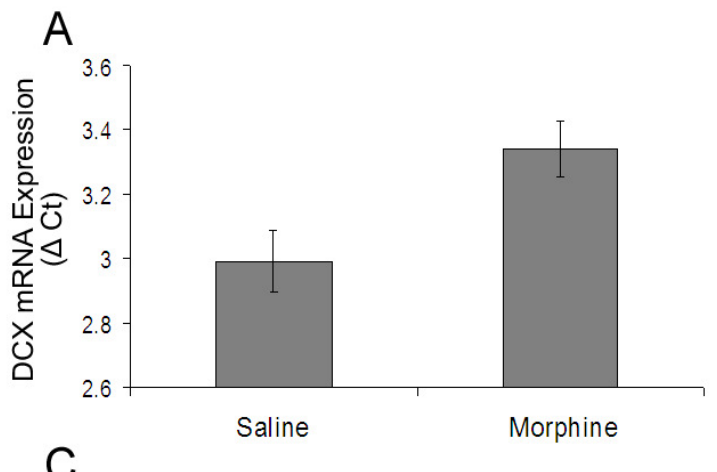

B
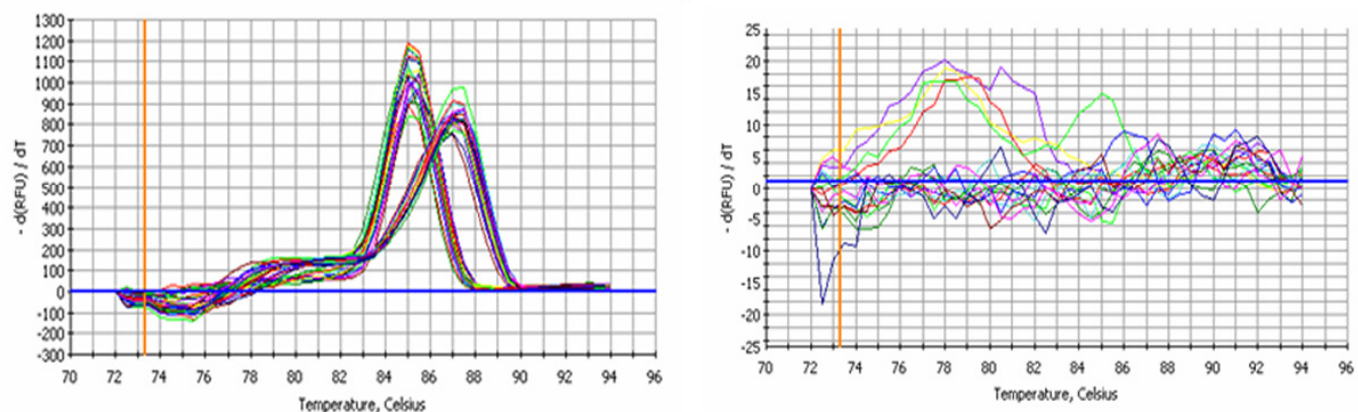

E

F
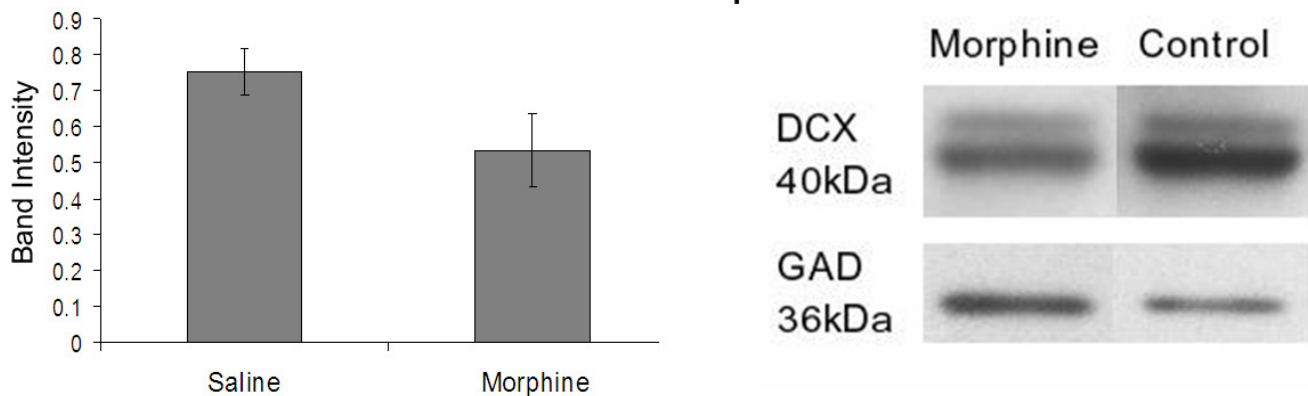

Fig. 6.8. Chronic morphine treatment during corticogenesis decreases DCX expression in the developing cortex. Chronic morphine treatment increases the $\triangle \mathrm{Ct}$ value at which DCX mRNA expression is detected in the E18.5 cortex $(\mathrm{P}=0.016$, Student's t-test, $\mathrm{n}=8$ saline-treated litters and 8 morphine-treated litters $)$ (panel A). Panel B shows PCR products electrophoresed on a 2\% agarose gel and stained with ethidium bromide. 
Lanes 1, 3 and 5 show DCX PCR product (187 bp) while lanes 2, 4 and 6 show cyclophilin A PCR product

(287 bp) that was amplified from the same reverse-transcribed template as the respective DCX PCR products.

Panel C shows a melt curve analysis in which $\mathrm{dRFU/dT}$ is plotted against reaction mixture temperature. DCX

PCR product was identified on the melt curve analysis graph as a peak at $87^{\circ} \mathrm{C}$, and cyclophilin A PCR

product could be detected as a peak at $85^{\circ} \mathrm{C}$. PCR products at the end point of the amplification reaction were pure except for small amounts of primer dimer, as shown by low levels of product detected at $76-83{ }^{\circ} \mathrm{C}$. Melt curve analysis of negative controls (PCR reactions conducted using either $\mathrm{ddH}_{2} \mathrm{O}$ or equimolar un-reverse transcribed RNA as template) showed no specific DCX or cyclophilin A amplification product (panel D). Panel E shows densitometric analysis of a western blot for DCX that was standardised using the internal control, GAPDH. Chronic morphine treatment decreased DCX protein, as was seen for DCX mRNA (panel A), although this was not statistically significant. Panel F shows DCX and GAPDH immunoblots that were used in the densitometric analysis of DCX protein expression. Double bands were present, and both bands were used for densitometry. Values on bar graphs are the mean \pm SEM.

Cortical lysates were western blotted for DCX in order to corroborate the real-time PCR datum. Immunoblotting for DCX protein showed a major and a minor band at approximately $40 \mathrm{kDa}$. These bands represent both the phosphorylated and unphosphorylated forms of the DCX protein (Francis et al., 1999). Densitometric analysis of both DCX bands, standardised against the internal control GAPDH (a single band at 36 $\mathrm{kDa}$ ), showed that chronic morphine treatment decreased the average amount of DCX protein, although this relationship was not statistically significant $(P=0.15$, Student's ttest, $\mathrm{n}=3$ saline-treated litters and 4 morphine-treated litters).

\subsubsection{Fas Expression}


To determine if the decreased levels of DCX could have been a result of Fas-mediated apoptosis, Fas mRNA expression was analysed using real-time PCR. As expected from the literature (Cheema et al., 1999), Fas mRNA expression was found to be extremely low in the same microdissected E18.5 cortex analysed for DCX mRNA expression. Fas mRNA expression was the same for saline-treated as for morphine-treated cerebral cortex when standardised using cyclophilin A as the internal control $(n=3$ saline-treated dams and 3 morphine-treated dams) (Fig. 6.9). This graph, however, omits data points from two morphine-treated litters in which PCR amplification failed to detect Fas mRNA in microdissected E18.5 cerebral cortex. This, in conjunction with the fact that Fas amplification products were typically detected at 31-32 reaction cycles, demonstrates that only trace amounts of Fas mRNA were present within the E18.5 cerebral cortex.

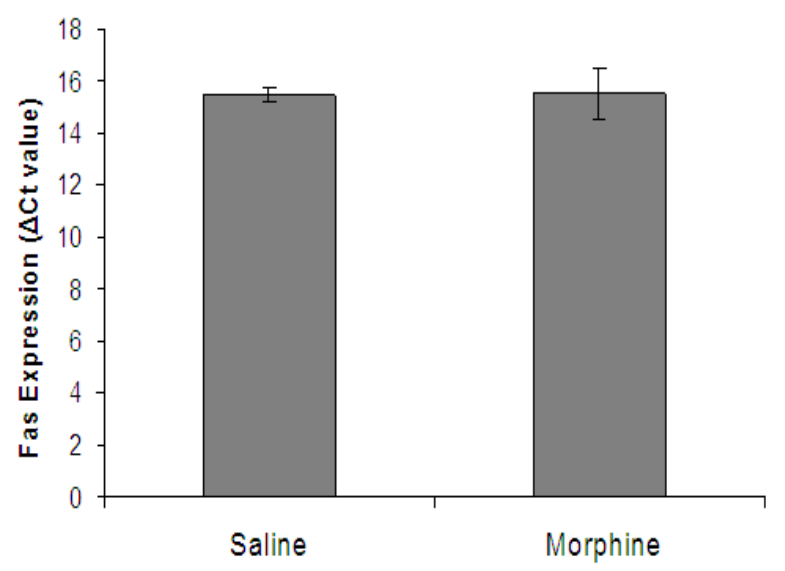

Fig. 6.9. Fas mRNA expression does not change between saline and morphine-treated E18.5 cerebral cortex. Fas mRNA expression (standardised to cyclophilin A mRNA expression) is not affected by chronic morphine treatment. Fas mRNA expression is also barely detectable in the E18.5 cerebral cortex. Two morphinetreated litters were omitted from this graph as Fas mRNA could not be detected from these cortical RNA extracts. ( $\mathrm{n}=3$ saline-treated litters and 3 morphine-treated litters). Values are mean \pm SEM. 


\subsubsection{Effect of Chronic Morphine Exposure on Cellular Proliferation and Neuronal}

Migration

Analysis of how chronic morphine treatment affects proliferation in the dorsal forebrain of the E18.5 mouse pup was measured by using a BrdU pulse at the end of chronic administration. Analysis of how chronic morphine administration affects neuronal migration from the $\mathrm{VZ}$ to the outside of the developing cortical plate was also analysed by injecting BrdU (50 mg/kg) at E15.5. Animals were allowed to survive until E18.5 and then were sacrificed. Unfortunately, due to difficulties in obtaining sufficient numbers of timedmated pregnant dams, analysis of the effects of chronic morphine on development of the cerebral cortex using BrdU pulse-chase paradigms was abandoned. However, preliminary results suggest that chronic morphine treatment had no major effects on total cellular proliferation (Fig. 6.10), as measured by BrdU pulse-labelling, and neuronal migration (Fig. 6.11) as measured by BrdU pulse-chase, in the developing cerebral cortex.
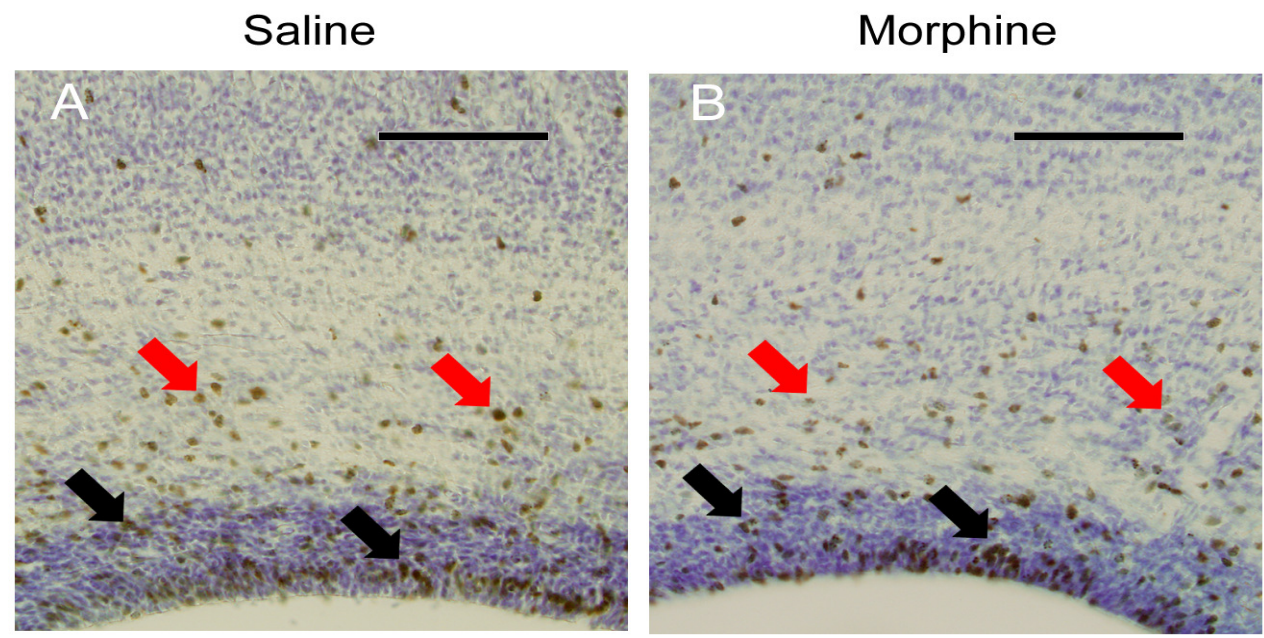
Fig. 6.10. Morphine does not have dramatic affects on total proliferation in the E18.5 dorsal forebrain.

Chronic morphine treatment (panel B) does not visibly decrease acute BrdU pulse-labelling (brown nuclei) within either the subependyma (black arrows) or the intermediate zone (red arrows) of the E18.5 forebrain compared with the saline-treated control (panel A). Sections were counterstained with thionin. Scale bars: 200 $\mu \mathrm{m}$.
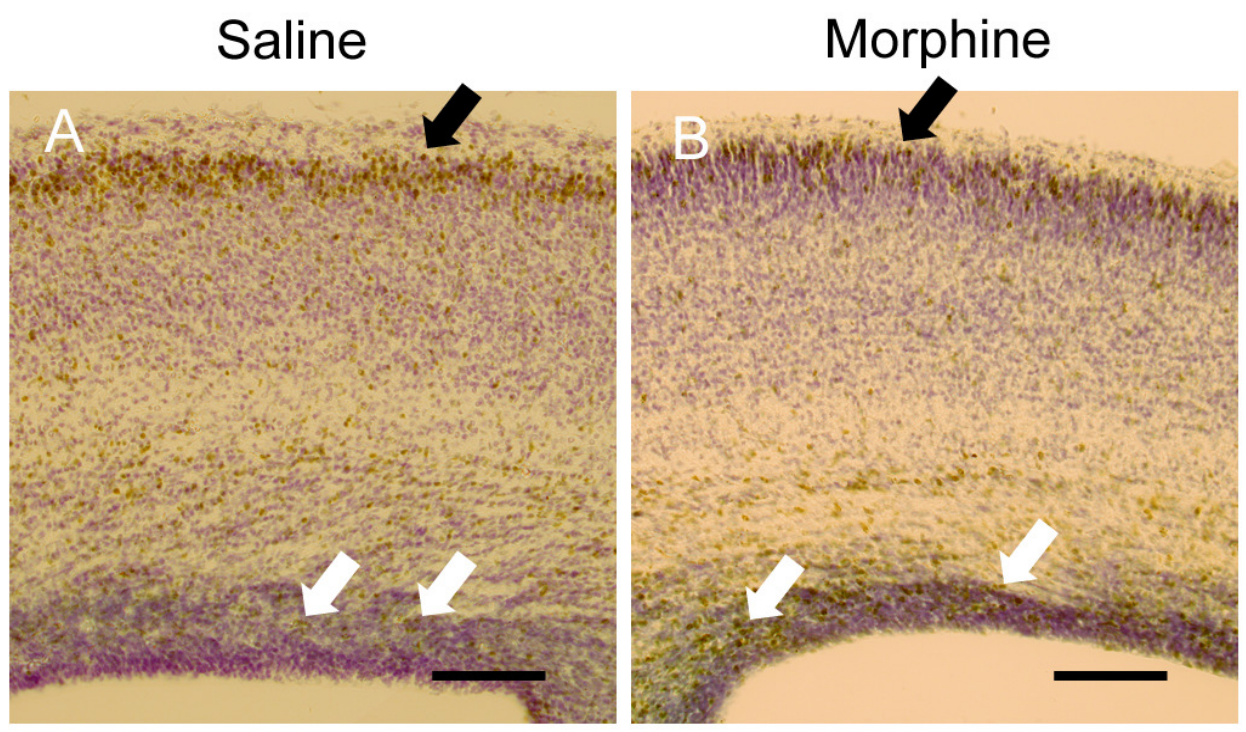

Fig. 6.11. Chronic morphine treatment does not induce major abnormalities in neuronal migration. Neuronal nuclei that were BrdU pulse-labelled at E15.5 (brown staining) had migrated to the edge of the cerebral cortex by E15.5 (black arrows). Some BrdU staining can be seen at the ventricular edge (white arrows). Chronic morphine treatment (panel B) does not cause major abnormalities, when compared with the saline-treated control brain (panel A). Scale bars: $200 \mu \mathrm{m}$.

\subsubsection{DCX-Positive Cell Counting in the E18.5 Cerebral Cortex}

Real-time PCR analysis showed that DCX expression in the cerebral cortex was lower in chronic morphine-treated animals. To determine the nature of this decrease in gene 
expression (i.e.: is it a reduction in the number of DCX-expressing cells or a reduction in DCX gene expression per cell?), E18.5 cortical sections were prepared and numbers of DCX-positive cells within the cerebral cortex were counted. At this stage, the entire cortex is positive for DCX staining. Only the pia and the SVZ show no DCX staining (Chapter 3). The same sections were also stained for NeuN to highlight the boundaries of the cerebral cortex. It was found that there was no difference in the number of DCX-positive cells within the E18.5 cerebral cortex, even though DCX expression had decreased (Fig. 6.12, panel A). Analysis of the number of DCX-positive neurons in the cerebral cortex and measurement of cortical thickness showed that even though morphine treatment had no effect, the region of lateral cerebral cortex analysed was larger than the medial cortex analysed $(\mathrm{P}=0.01)$.
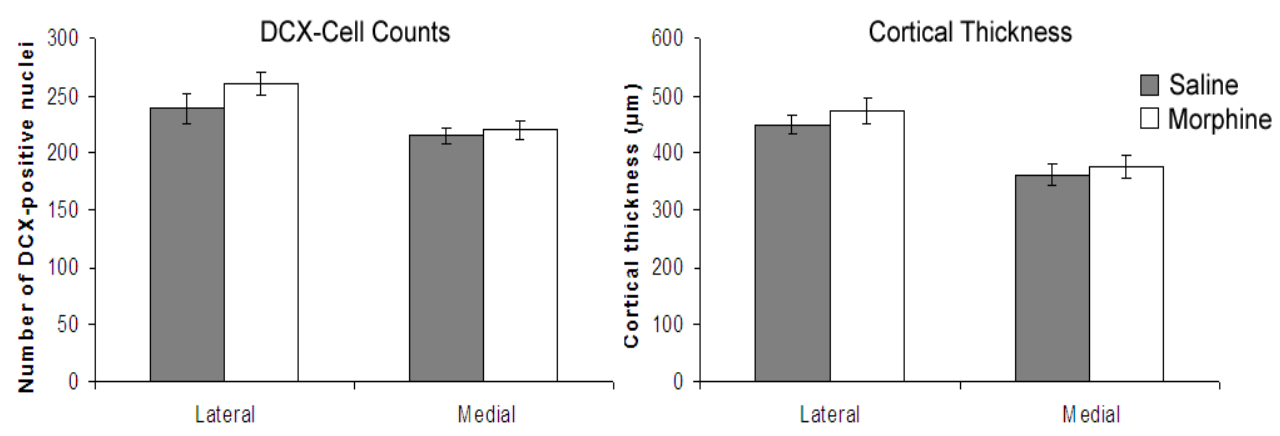

Fig 6.12. DCX-positive cell counting and thickness of the E18.5 cerebral cortex. Panel A shows the number of DCX-positive DAPI-stained nuclei per counting column. Grey bars show the number of cells counted in the lateral cortex as opposed to cells counted in the medial cerebral cortex (white bars). There is no difference in the number of cells counted for each region of cortex between saline-treated controls and morphine-treated animals. There is however a slight decrease in the number of cells counted in the medial cerebral cortex as opposed to the larger lateral regions of cortex. Panel B shows the average cortical thickness of the same regions of cortex that were counted for DCX-positive cells. These measurements show the same patterns as 
the DCX-positive cell counts. Grey bars show cortical thickness in the lateral cerebral cortex and white bars show cortical thickness in the medial cerebral cortex. $n=4$ saline-treated dams and 4 morphine-treated dams. Values are the mean \pm SEM.

\subsubsection{Analysis of the P5 Cerebral Cortex - Western Blotting}

To determine whether a change in neuronal content as a result of chronic morphine treatment could be detected by western blotting, P5 mouse neocortex was microdissected, homogenized and electrophoresed on an acrylamide gel. Western blotting was carried out for the neuronal markers, NeuN and GAD 65+67, the glial marker GFAP and the housekeeping gene, GAPDH (Fig. 6.13). Interestingly, no change in protein levels for the neuronal and glial markers tested could be detected $(n=4$ saline-treated animals, 4 morphine-treated animals (all from different litters)), despite the decrease in DCX mRNA observed after chronic morphine treatment at E18.5. All values were normalized against the housekeeping gene, GAPDH. 


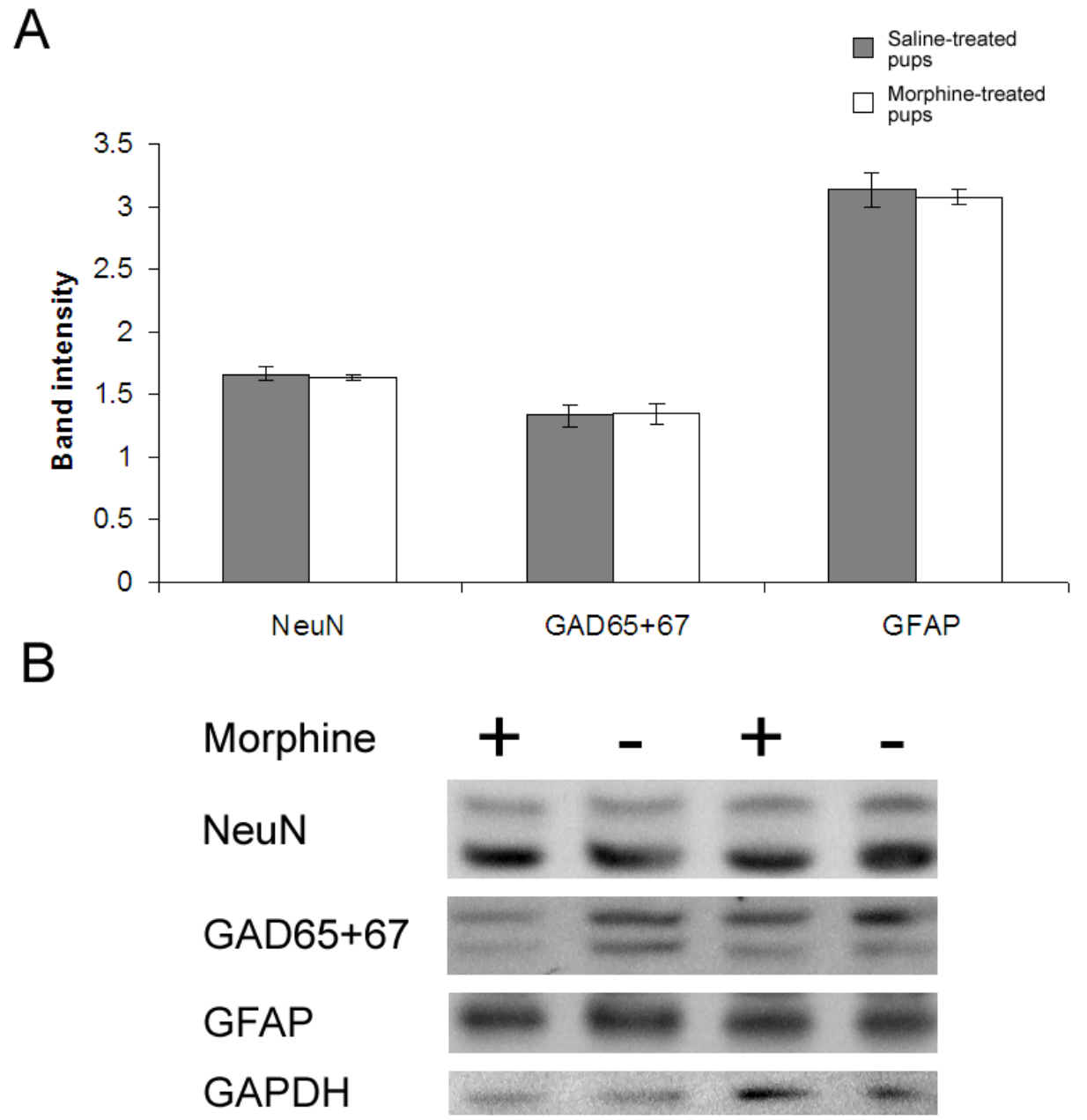

Fig. 6.13. Chronic morphine exposure from E11.5 to E18.5 does not change expression of neuronal and glial markers in the cerebral cortex at P5. Protein expression of the mature neuronal marker, NeuN, the GABAergic neuronal marker, GAD65+67, and the astroglial marker, GFAP, was not altered by chronic morphine treatment when standardised to GAPDH (panel A). Panel B shows specific immunostaining taken from fluorescent scans of four representative P5 cerebral cortex western blots. $n=4$ saline-treated pups and 4 morphine-treated pups (from different litters). Values are the mean \pm SEM.

6.3.6 Stereological Cell Counting in the P5 Neocortex 
To analyse the cellular content of the P5 neocortex, thick paraffin embedded coronal sections of P5 brains that were chronically treated with either saline or morphine in utero were Nissl stained with thionin and counted via a stereological method to estimate the total number of nuclei that bore neuronal characteristics, the neuronal density and the reference volume of the cortical layers in question. Stereological measurements were taken from the supragranular layers (II, III and IV), layer V and layer VI.

Using a repeated measures analysis, it was found that chronic morphine treatment from E11.5-E18.5 had a significant interaction with cortical layer number within the neocortex of the $\mathrm{P} 5$ pup $(\mathrm{P}<0.05)$. Chronic morphine treatment had the largest impact on layer $\mathrm{V}$ of the neocortex causing a decrease in the number of neurons in this layer (Fig. 6.14, panel A). Using Cavalieri estimates, it was shown that morphine had little affect on the volume of the neocortical layers (panel B). The reduction in cellular number within the neocortex was therefore primarily a result of lower cell density and not a decrease in the volume of layer V. 


\section{A}

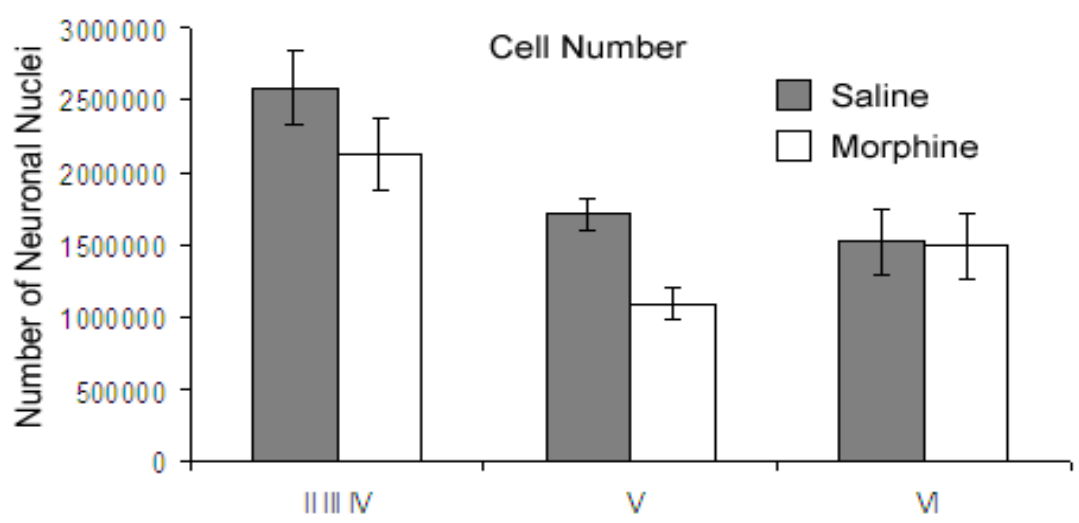

B

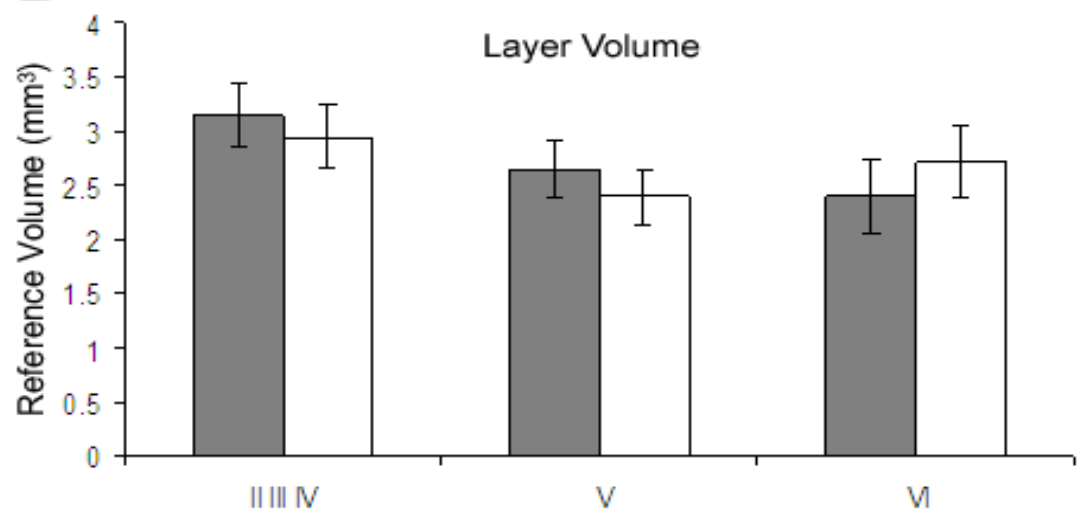

Cortical layer

Fig. 6.14. Stereological analysis of the cerebral cortex of the P5 mouse. Panel A shows that chronic in utero morphine treatment had little effect on the numbers of neurons in the supragranular layers (layers II, III and IV). Chronic morphine treatment did markedly decrease the numbers of neurons in layer V of the P5 cerebral cortex $(\mathrm{P}<0.05)$. Layer VI did not display any change. Panel B shows that although morphine impacted on the numbers of neuronal nuclei in layer $\mathrm{V}$, it did not affect the volume of the cortical layers. $\mathrm{n}=4$ salinetreated animals and 3 morphine-treated animals (layers II/III/IV and layer VI); 3 saline-treated animals and 5 morphine-treated animals (layer V). Values are the mean \pm SEM.

\subsection{Discussion}


Collectively, the current experiments showed that chronic morphine treatment, in utero, perturbed corticogenesis without having any effect on the body weight of the animals. The amount of DCX transcript in the E18.5 cortex was decreased $(\mathrm{P}<0.05)$, and, at P5 a reduction in the number of cells was detected that was layer specific $(\mathrm{P}<0.05)$.

\subsubsection{Chronic Morphine Administration does not Decrease Weight in the Perinatal Mouse}

The interesting observation that morphine treatment of the pregnant dam had an effect on maternal weight $(\mathrm{P}=0.01)$ could have a number of explanations. It was observed that morphine treatment produced opiate-induced hyperlocomotion, as has been described in the literature (Mori et al., 2004). This extra activity may have reduced weight gain in the pregnant dam due to increased energy consumption. Alternatively, or in conjunction with morphine-induced hyperlocomotion, morphine may have disrupted eating habits in the pregnant dam, as previously documented in the rodent (Markham et al., 1971). No monitoring of food intake was attempted in this study.

Importantly, although chronic morphine treatment affected weight gain in the pregnant dam, it did not affect the weight of the E18.5 mouse foetus or P5 pup. When pups were cross-fostered between treatment groups at birth it was observed that exposure of the pups themselves to morphine in utero had absolutely no effect on pup weight at P5. However, pups that were raised by saline-treated mothers, whether the pups had developed in a morphine or saline-treated mother, were lighter than pups that were raised by morphine- 
treated mothers, even though morphine treatment of the mother had ceased 5 days earlier. The reason for this difference remains unclear, although it is possible that the smaller litter size of the morphine-treated mothers (an average of 5.4 pups, as opposed to 6.4 pups for a saline-treated dam's average litter size) resulted in higher pup weights for morphine-treated mothers. However, it is interesting to note that human opiate exposed mothers show significantly increased feeding behaviour towards their infant (LaGasse et al., 2003). Taken together, little biological significance should be assigned to the statistical significance of the increase in pup weights observed in litters raised by morphine-treated dams. The lack of effect observed between morphine treatment in utero and pup weight contrasts with several studies in the literature, which show significant decreases in weight associated with prenatal methadone exposure (Buchenauer et al., 1974; Chandler et al., 1975; Hutchings et al., 1976; Zagon et al., 1977b) and morphine exposure (Tao et al., 2001). This disparity between our results and the literature is most likely a result of differences in dosage as well as duration of treatment. However, our results do indicate that long-term prenatal exposure to the opiate, morphine, does not necessarily result in reduced pup weight, a situation also seen in pregnant human opiate abusers (Lester et al., 2002).

\subsubsection{Morphine Induced Down-Regulation of DCX Expression}

Although chronic morphine treatment showed a lack of general growth effects, as measured by body weight, morphine decreased the amount of DCX mRNA expression within the cerebral cortex at the end of neurogenesis. This result may have one of two explanations. Either DCX mRNA was down-regulated in each cell by morphine treatment, or morphine 
decreased the proportion of DCX expressing cells within the dissected volume of cortical tissue. The fact that no significant difference was detected between the numbers of DCX expressing cells in the E18.5 mouse cortex treated with morphine and saline suggests that the second explanation (a lowered DCX expression per cell) is the most likely cause of the decrease in DCX expression.

There are a number of explanations for why DCX expression may be lower in morphinetreated animals per migrating neuron. Morphine may be directly or indirectly signalling to DCX positive neurons to decrease DCX expression. Direct signalling is unlikely since migrating neurons do not express MOR (Chapter 3). Another explanation for the loss of DCX mRNA expression in the E18.5 forebrain is that morphine treatment may have caused apoptosis of DCX-positive neurons. Morphine treatment and withdrawal is known to cause apoptosis and up-regulation of Fas and FasL proapoptotic genes in other systems (Yin et al., 1999; Emeterio et al., 2006). However, in the late foetal mouse brain, Fas expression was barely detectable (Cheema et al., 1999; Fig. 6.9), and morphine had no effect on these levels in the late foetal cortex. It is unlikely that Fas-mediated apoptosis accounts for the decreased levels of DCX mRNA expression seen with chronic morphine treatment.

Alternatively, morphine may perturb the development of radial glial cells in the VZ such that by E18.5, more radial glia in the developing forebrain have finished neurogenesis, and therefore there is less migratory activity and less DCX expression to be detected via realtime PCR. It is conceivable that morphine treatment shifted maturation of the cortex. Given the short time in which the cortex is generated, a shift by even half a day could have 
accounted for the observed reduction in DCX. Indeed, the intrinsically controlled programme of neurogenesis followed by neuronal progenitor cells (Shen et al., 2006) can be precociously matured by changing the surrounding cellular environment (McConnell, 1988). In line with this, it is also interesting that morphine can cause premature differentiation of astrocytes cultured from the P1-2 mouse cortex (Stiene-Martin et al., 1993). Given this evidence, the possibility that morphine treatment perturbs maturation of the neurogenic programme by modulating maturation/differentiation in astrocytic radial glia should be the focus of future research.

\subsubsection{Chronic Morphine and Loss of Cortical Neurons in the Postnatal Pup}

Hammer and Seatriz (1993) showed that chronic, continuous morphine treatment in the developing rat (E12-P6) decreased neuronal number in the cerebral cortex. To test for this in mice, western blot analysis of neuronal (NeuN and GAD65+67) and glial (GFAP) markers in the P5 mouse brain was conducted. Surprisingly, it was found that chronic morphine treatment over the period of corticogenesis did not change levels of neuronal or glial markers in the cerebral cortex. This meant that by P5, any difference in neuronal marker expression detected at the end of gestation had either been compensated or corrected for by the developing brain, or western blotting was too insensitive to pick up a difference. The latter explanation is a distinct possibility because of the loss of spatial information following homogenisation. 
To determine if any differences in cortical architecture due to chronic morphine treatment persisted (or emerged) in the P5 brain, 6 days post-morphine exposure, stereological analysis of the different cortical layers was performed. Seatriz and Hammer (1993), using Nissl staining in the rat, found a decreased number of neuronal nuclei after chronic morphine exposure from E12 to P6. Interpretations in this study were limited by the broad window of morphine treatment used. Early in that same window (E14-E20), histogenesis of the cerebral cortex occurs in the rat. However, in the neonatal period (P0-P6), large numbers of neurons in the cerebral cortex (10-20\%) undergo apoptosis (Verney et al., 2000) as the brain develops its pattern of synapses and neuronal circuitry. Stereological analysis in the present study supported the findings of Seatriz and Hammer (1993) as chronic morphine treatment in the developing mouse brain reduced the number of cortical neurons. This only seemed to occur, however, in layer V of the neocortex, although the experiment may not have had the power to detect changes in other layers. Seatriz and Hammer (1993) also showed that layer V was most affected by chronic morphine treatment. We only treated with morphine from E11.5 to E18.5 in the mouse, a period that corresponds to the time of corticogenesis. From these data as well as the fact that chronic morphine treatment did not decrease numbers of DCX-expressing cells (neurons) in the cerebral cortex at the end of gestation (E18.5), it is probable that chronic morphine treatment in utero increased apoptosis in the neonate. This idea is consistent with the fact that the volume of the P5 cerebral cortex was unaltered. Also, Harburg et al. (2007) show that MOR KO augments neurogenesis in the adult hippocampus by increasing the number of surviving neurons, demonstrating that MOR may modulate apoptosis in the CNS. If it is the case that in utero morphine exposure increases apoptosis in the postnatal cortex, it is 
interesting and significant that morphine has far reaching impacts that are still evolving long after morphine treatment has ceased.

Neurons that reside in the infragranular layers of the cerebral cortex (layers V and VI) are a major output for the cerebral cortex that modulates motor and sensory function (Hattox and Nelson, 2007). Projection neurons of layer V are a heterogeneous population that can be divided into two groups, type I, which include the large corticospinal neurons (Gao and Zheng, 2004), and neurons which project to the pons, and type II, which project to the ipsilateral striatum or the contralateral cortex. Such neurons can also be subdivided by the use of molecular markers (Molnar and Cheung, 2006). Layer V corticofugal neurons are also crucial to movement. Beloozerova et al. (2003) showed that layer V corticofugal neurons, not layer V cortico-cortico or layer VI neurons, were highly active during both simple and complex locomotion. Interestingly, decreased psychomotor performance has been linked to in utero opiate exposure in humans (Bunikowski et al., 1998). This is controversial as other research has shown this to be the result of confounding environmental factors (Hans and Jeremy, 2001). The largest study on prenatal drug exposure in humans to date, found that without correction for covariates, opiate exposure in utero depressed psychomotor performance (Messinger et al., 2004). With correction for covariates, this effect became statistically insignificant $(\mathrm{P}=0.07)$.

Our study has implications for opiate exposure on the human foetal brain since it showed that development of the cerebral cortex was sensitive to opiates over the period of corticogenesis, as morphine affected radial glial cell cycle progression, and postnatally, 
apoptosis. Even though in utero exposure to morphine has effects on neurons both at the end of neurogenesis (E18.5), and in the neonatal mouse (P5), it remains to be determined whether or not these changes persist into adulthood. It has been shown that much neuronal apoptosis occurs after P5 (Verney et al., 1999). It has also been shown that neuronal apoptosis occurs in response to a lack of neurotrophic support from target neurons or cells (Raff et al., 1993; Davies, 2003). Fewer neurons at P5 may simply mean that the neurons that do exist have a higher chance of forming meaningful synaptic connections and thus have a higher chance of survival. Future studies should therefore be extrapolated to older developmental time points as well as into adulthood. Furthermore, even if morphineinduced changes in the cerebral cortex persist into adulthood, it does not necessarily mean that the animal will be detrimentally affected at a behavioural level. For example, in the development of Parkinson's disease, a human can sustain an $80 \%$ decrease in striatal dopamine before becoming symptomatic. It is thought that plasticity in cortico-basal ganglia-thalamocortical pathways may account for substantial compensation to dopaminergic dysfunction in Parkinson's disease (Bezard et al., 2003).

In conclusion, the current chapter has provided evidence that chronic morphine exposure in utero decreases DCX expression in the E18.5 cerebral cortex. This may be a result of a perturbation in the neurogenic programme, since a decrease in the number of neurons was not detected. Chronic morphine exposure in utero also has lasting implications to neonatal development. At P5, after morphine exposure had ceased, neuronal loss was detected in layer $\mathrm{V}$ of the cerebral cortex. It is unlikely that these effects were the result of system-wide 
toxicity since no biologically significant changes in either foetal or postnatal pup weight were detected. 


\section{General Discussion}

\subsection{Project Summary}

The overall aim of this thesis research was to characterise the effects of opiate exposure within the context of the developing cerebral cortex. Specifically, this was investigated by characterising the localisation of MOR in the foetal brain, determining the effects of morphine exposure on the proliferative populations of neuronal progenitors within the developing VZ, and assessing the effects of chronic, or long term opiate exposure on development of the cerebral cortex. We have demonstrated that the opioid signalling system can impact upon development of the cerebral cortex. This was shown by linking MOR IR with the neuronal progenitor cells, radial glia. MOR IR was found in the neurogenic (E15.5) as well as the gliogenic (E18.5) cortical neuroepithelia. The same MOR-staining that was seen in the E18.5 mouse forebrain was also seen in the foetal human brain at mid-gestation (16 and $19 \mathrm{GW})$. Although no direct effect on net proliferation could be demonstrated, it was shown that acute exposure to morphine could impact upon progression of the cell cycle of progenitor cells $\left(\mathrm{G}_{2} / \mathrm{M}\right.$ phase $)$ within the E15.5 mouse forebrain. Finally, having demonstrated opioid receptors were present within proliferative zones of the foetal forebrain, and progenitor cells were sensitive to opiate exposure, it was shown that chronic opiate exposure could affect development of the cerebral cortex itself. Prenatally, these changes were subtle but supported the idea that 
morphine may have perturbed developmental progression. Postnatally, however, chronic morphine exposure, in utero, resulted in fewer neurons in the cerebral cortex. These results indicate that development of the cerebral cortex is sensitive to opiate signalling. This research is significant because it is the first research that directly demonstrates morphine has the potential to modulate cortical progenitor cells (radial glia) in utero. These data also support the idea that opioid signalling plays an endogenous role in the developing brain, although this assertion requires further research.

\subsection{Future Research}

\subsubsection{Remodel the In Vivo Experiments in an In Vitro Setting}

Working in whole animals is advantageous because the results that are obtained are not confounded by abstract molecular and physical environments. The primary disadvantage to in vivo research is that an animal is a complex system that is difficult to dissect at the molecular and biochemical level. Differential distribution of any given compound within an animal as well as the large amounts needed to treat animals (versus treatment of a culture dish of cells) are both factors that favour the use of in vitro systems for detailed analysis of biochemical processes. With these considerations in mind, future research should include organotypic slice culture in an investigation of the effects of opioids on development of the VZ. 
Organotypic slice culture has several advantages over in vivo studies for investigating development of the VZ in that it has all of the benefits of in vitro work yet it maintains some of the normal local cell interactions and phenomena like INM and neuronal migration along radial processes. Radial glial INM, cell cycle and mitotic stages can be visualized in slices using fluorescent DiI labels (Miyata et al., 2002). These lipophilic fluorescent dyes label plasma membranes of living cells. Unfortunately, organotypic explants of VZ exhibit a slower cell cycle time than the $\mathrm{VZ}$ in vivo, as well as transient checkpoints at $\mathrm{G}_{1}$ and $\mathrm{G}_{2}$ phase which disappear several hours after explant (Takahashi et al., 1999). However, the use of organotypic slice culture allows for the unrestricted use of opioid antagonists as well as biochemical tools like specific kinase and calcium store inhibitors. These could help to dissect the signalling mechanisms that lead to the morphine-induced slowing of cell cycle in the $\mathrm{VZ}$ observed in vivo. A good beginning for research on opioid signalling and intracellular signal transduction in organotypic slice cultures would be to test the work of Coscia and colleagues, which centres on transactivation of EGF receptors and the MAP kinase pathway (Belcheva et al., 2003; 2005). Opioid effects on proliferation that signal through the MAP kinase pathway have also been shown in other systems such as in hippocampal progenitor cells (Persson et al., 2003a).

\subsubsection{Use Opioid Antagonists to Identify Receptor Subtypes and Endogenous Activities}

Many studies that have investigated the effects of opiates on proliferation have used antagonists such as naltrexone to demonstrate that signalling occurs through the opioid receptors (Hauser et al., 1996; 2000; Stiene-Martin et al., 2001). 
A few studies have demonstrated that some opioid peptide mediated effects cannot be antagonised by the opioid receptor antagonists, naltrexone and naloxone (reviewed in Wollemann and Benyhe, 2004). However, all studies (to the knowledge of the author) that have attempted to antagonise proliferative or developmental effects of opioids with opioid receptor antagonists have been able to do so successfully. The use of opioid antagonists would serve to refine the current research twofold. Selective opioid antagonists (Table 7.1) would enable a more precise description of the specific opioid receptors that modulate corticogenesis under normal conditions. Even though the present study has produced supporting evidence for a role of the opioid system in corticogenesis by showing that radial glia express opioid receptors and are sensitive to morphine, it is still possible that the effects observed in this study are pathological effects induced by supranormal opiate levels that bear little relevance to normal physiological conditions.

Opiate antagonists were not used in the current research project because of the limited supply of animals, especially pregnant dams, which allowed for morphine tests but not antagonist tests. The decision was made to more thoroughly investigate the effects of the opiate morphine on brain development rather than to investigate fewer possibilities but with opioid antagonists included as well. To gain the full benefit from using antagonists, one would employ a battery of receptor-subtype specific antagonists. Given the available resources, this would have severely restricted the breadth of this thesis research. 


\begin{tabular}{|c|c|c|}
\hline Opioid Receptor & Antagonist & Notes \\
\hline \multirow{3}{*}{ MOR } & CTOP & Highly selective mu antagonist, cyclic peptide \\
\hline & CTAP & Selective mu antagonist, cyclic peptide \\
\hline & Beta-FNA & Irreversible selective mu antagonist, non-peptide \\
\hline DOR & Naltrindole & Highly selective delta antagonist, non-peptide \\
\hline \multirow{3}{*}{ KOR } & Nor-BNI & Selective kappa antagonist, non peptide \\
\hline & DIPPA & Irreversible, selective kappa antagonist, non peptide \\
\hline & GNTI & Highly potent, selective kappa antagonist, non peptide \\
\hline
\end{tabular}

\subsubsection{Use Gene KO Animals to Delete Specific Receptors}

A more precise tool for the elucidation of the opioid receptor population involved in the morphine effects would be to use different as well as combinatorial opioid receptor KO animals. In this thesis, we used a MOR KO to provide evidence that the morphine-induced effects on INM were MOR-related. KOs eliminate the unknown aspects inherent in pharmacological receptor blockade such as receptor promiscuity and unknown accessibility of the antagonist to the foetal brain. However, even $\mathrm{KO}$ mice have their caveats in developmental research (see Chapter 1 for detailed examples). The best way to study how opioid receptors may contribute towards CNS development would be to employ an inducible KO, such as the tamoxifen inducible gene KO (Erdmann et al., 2007). A tamoxifen inducible gene $\mathrm{KO}$ is a conditional $\mathrm{KO}$ in which Cre-recombinase is expressed as a fusion protein with a mutated ligand domain of an estrogen receptor. Cre-recombinase activity is initiated by injecting tamoxifen into the animal. This Cre-recombinase activity will then induce recombination of DNA flanked by loxP sites. As recombination can be 
temporally restricted by use of the tamoxifen system, it can also be spatially restricted by placing Cre-recombinase expression under the control of a cell-specific promoter. This system has been used before with success in the embryonic mouse brain (Burns et al., 2007). To continue the research of the present study, loxP sites would be placed around any or all of the opioid receptor genes, and Cre-recombinase expression would be placed under the control of the radial glial-specific BLBP or hGFAP promoter. Injection of tamoxifen would therefore induce opioid receptor $\mathrm{KO}$ that is specific to radial glia. This approach has the technical advantages of both molecular biology and pharmacological receptor blockade in that specific receptors can be removed acutely, without time for the developing system to induce compensatory measures.

\subsubsection{Investigate Different Treatment Regimes - Continuous Administration by Osmotic}

\section{Pumps}

Further research should also expand upon and refine drug administration regimes. Continuous release of opiates with miniosmotic pumps show reduced toxicity with chronic experiments (Hutchings et al., 1992). This does not matter so much with acute experiments, where the animal remains under the influence of the opiate for the duration of the experiment; however, it would be useful for longer duration treatments such as exposure over the entire period of corticogenesis. The use of a constant infusion of opiate in a chronic experiment would be beneficial as the animal would not withdraw from the injected opiate drug after it has been cleared from its system. The use of osmotic pumps would be a 
good model for opiate administration in hospital where opiates may be infused intravenously or epidurally (George, 2006)

Further research into cell cycle progression and chronic opiate administration is also required. The current study found acute courses of morphine slowed $\mathrm{G}_{2} / \mathrm{M}$ phase progression. Research into whether this occurs after chronic morphine exposure, or if some adaptive measures have occurred, would be of clinical relevance. Other studies have found that chronic morphine exposure markedly decreases proliferation in the brain, unlike acute exposure (Eisch et al., 2000). Future research should investigate whether such dramatic differences in response to chronic or acute drug exposure is a consistent theme in the developing brain.

\subsubsection{Use Other Clinically Relevant Opiates}

As well as mode of administration, the use of different opiates of clinical significance should also be considered. These include fentanyl, alfentanyl, codeine, pethidine, diamorphine (heroin) and methadone. Although morphine is an opiate of extremely high clinical significance, the fact that it does not induce opioid receptor internalisation but other opiates do (Arden et al., 1995; Keith et al., 1996) means that the effects of morphine described in this thesis should be examined using other opiates. Furthermore, different splice variants of MOR have been shown to have different affinities for different opioid ligands (Bolan et al., 2004). Whereas it is valid to say further research with selective antagonists is important for elucidation of a role for opioids in normal development, it is 
just as valid to say future research should focus on the use of additional opiate compounds and administration regimes to strengthen its clinical relevance.

\subsection{Weaknesses in the Field of Opioids and Developmental Biology}

The study of how opiate drugs affect development had its roots in the panic that surrounded the recreational abuse of heroin and the use of methadone as a maintenance drug (Markham et al., 1971). Early morphometric studies found no significant effects of opiates on growth, apart from mild decreases in weight. It was not long after this, however, in the 1980s before people began to investigate potential effects of opiates on cellular proliferation, using tools such as $\left[{ }^{3} \mathrm{H}\right]$ thymidine (Vertes et al., 1982; Miller et al., 1982; Zagon and McLaughlin et $a l ., 1983)$, and these continued with the characterisation of the opioid system in astroglia in the late 1980s and early 1990s (Vilijn et al., 1988; Hauser et al., 1990; Stiene-Martin et al., 1991a). Over the 1990s, research that came mainly out of three labs (those of I. Zagon, K. Hauser and C. Coscia) now provide the bulk of the literature on how the opioid system interacts with neural development at a cellular scale. Apart from the lab of C. Coscia, which is investigating how opioids interact with the MAP kinase signaling pathway in glia (Belcheva et al., 1998; 2000; 2002; 2003; 2005), these studies on how opioids affect the proliferation of astroglial lineage cells have largely come to a halt. The reason why this research has stopped, in my opinion, is in part due to difficulty in reproducing some of the work that has been previously published. To some extent, this may be due to the context dependency and temporal sensitivity of opioid effects on development, possibly due to the 
inherently indirect nature of opioid signaling on cellular proliferation via receptor tyrosine kinases (discussed further in Chapter 1). However, on a more aggressive note Petrie (1993) labeled the study of Zagon and McLaughlin (1983) as "lab specific", having failed to replicate the reported growth-enhancing effects of opioid antagonism with naltrexone. We also had difficulty in replicating results published in the study of Reznikov et al. (1999). Although we noted that different opioid ligands were used (Chapter 5), we still failed to observe the $20-40 \%$ changes in S phase-labelling reported in the Reznikov study.

These points aside, one particular aspect of the history of research on opioids and development seems curious. Results from some studies have reported massive effects on the number of cells in given brain structures. These include a 2.7 fold increase in the number of neurons in the molecular layer of the cerebellum in response to postnatal high dose naltrexone treatment (Zagon et al., 1983), a 28\% decrease in the number of internal granule neurons of the cerebellum in response to postnatal low dose naltrexone (Zagon et al., 1986a) (a tremendous decrease in neuronal number given the internal granule layer houses half of the neurons in the brain (Ghez and Thach, 2000)) and a $40 \%$ increase in the $\left[{ }^{3} \mathrm{H}\right]$ thymidine labeling index of the E16 (c.f. E15 in the present study) mouse in response to an acute dose of DAMGO. Given the magnitude and significance of these results, it is unclear why the opioid system in the developing cerebellum has not been vigorously researched and widely acknowledged as a major modulating force in the proliferating cerebellum. It is also unclear as to why a signaling system that could increase the labeling index of the VZ by a massive $40 \%$ has not been pursued, given that corticogenesis is now an intense topic of study. Such a large induction of S phase-labeling seems unusual. To put 
the sheer magnitude of a $40 \%$ increase in $\mathrm{S}$ phase labeling into perspective, overexpression of insulin-like growth factor in the mouse telencephalon increases cell cycle reentry by 15\% (Hodge et al., 2005) while EGF (20 ng/ml) increases BrdU labeling in cultures of SVZ cells by $40 \%$ (Agasse et al., 2006). Furthermore, a $40 \%$ increase in S phase labelling along with reported increases in mitotic indices at the ventricular edge in response to DAMGO (>20\%) (Reznikov et al., 1999) would provide a major disruption to the model of corticogenesis put forward by Caviness et al. (2003) and would therefore be likely to cause obvious and long lasting morphological effects on cortical histogenesis.

Despite the uncertainty of these large opioid induced changes, the present study provided some support for major effects similar to those reported in the literature. For example, Seatriz and Hammer (1993) showed that chronic morphine exposure reduced cortical layer V neuronal number by half in the postnatal pup. This was also demonstrated in the mouse (Chapter 6) where a similar effect was observed. Given that this effect was reproducible, why has such a large effect gone unnoticed in the literature? Perhaps this wane in opioiddevelopmental research has come as a consequence of a number of studies pointing out in utero exposure to opiates in humans produces only weak effects, at best, on human brain development (Kaltenbach, 1994).

An area that needs clarification is the importance of OGFr in brain development. The nuclear met-enkephalin receptor or 'opioid growth factor receptor', is a receptor that reportedly has anti-cancer potential (Cheng et al., 2007; 2008) and globally modulates cellular proliferation during organogenesis (Zagon et al., 1999b). The literature on this 
receptor comes from mostly one lab (Zagon and colleagues), and it would be useful to have more molecular and biochemical background on the action of the receptor. Since the cloning of OGFr in 1999 (Zagon et al., 1999a), 31 papers have been published on or relating to that receptor (search "opioid growth factor receptor" on Pubmed Entrez). Of these 31 papers, only 5 are from groups other than Zagon's laboratory group. Of these 5 papers, 3 relate to global gene expression studies (Urosevic et al., 2004; Matejusova et al., 2006; Oczko-Wojciechowska et al., 2006), one to cDNA expression library screening (Mollick et al., 2003) and one is an immunohistochemical study that used antibodies provided by Zagon and McLaughlin (Robertson and Andrew, 2003). Thus, none of these 5 studies provide support for a direct proliferative function of the cloned receptor. This contrasts greatly with the multi-lab corroboration that was immediately forthcoming after cloning of the classical opioid receptors (Evans et al., 1992; Kieffer et al., 1992; Chen et al., 1993a; 1993b; Eppler et al., 1993; Li et al., 1993; Meng et al., 1993; Minami et al., 1993; Thompson et al., 1993; Wang et al., 1993). A quick search on Medline reveals that the last 20 papers published on other developmentally associated proteins, specifically the TrkA neurotrophin receptor, notch1 and wnt7b consisted of studies from 19, 20 and 19 different labs, respectively. Such numbers are indicative of a healthy research field with findings that are readily reproducible.

Optimistically, research on opioid signalling and brain development has available several new opportunities. If opioid research can develop a niche within the field of brain development, it will be able to benefit from large gains in knowledge made since the early 2000s. The significance of opioid-developmental research obviously extends to the clinical 
arena; however, future research should perhaps focus on the endogenous biology of the opioid system in development. This approach has excellent potential as there is much opportunity to marry the knowledge already gained on opioidergic modulation of astrocytic proliferation with new-found roles for astrocytic lineage cells. The latter range from radial glia, which manufacture the neurons of the CNS, to astrocytes, which assume the role of stem cells within the adult brain.

\subsection{An Integrated Model of Opioidergic Signalling and Cortical Development}

Based on data taken from this project, the following hypothetical model of how opiates impact on corticogenesis, in vivo, can be made.

Opioid receptors are expressed on neuronal progenitor cells of the cerebral cortex (Chapter 3 and 4). Evidence for direct opioid signalling impacting on proliferation of astroglial lineage cells is presented by in vitro studies (Stiene-Martin and Hauser, 1993; Hauser et al., 1996). Opioid signalling through MOR (possibly also KOR) on radial glial cells slows cell cycle progression in progenitor cells of the VZ. This occurs in vivo and may be restricted to $\mathrm{G}_{2} / \mathrm{M}$ phase of the cell cycle (Chapter 5). Acting through a perturbation of the cell cycle, opioids modulate progression of the intrinsic neurogenic programme to induce early maturation. This is a distinct possibility as a change in cell cycle time and INM alters the length of time a radial glial cell spends in an environment that may or may not favour differentiation (discussed in Chapter 5). Other studies show the intrinsic neurogenic 
programme may be influenced by external environmental cues to promote precocious maturation (McConnell, 1988) and that morphine influences differentiation in astrocytic cells (Stiene-Martin et al., 1993). The fact that less DCX (migratory marker) was expressed at the end of neurogenesis in response to chronic morphine treatment suggests that morphine throughout corticogenesis impacted upon the developmental maturity of cells within the VZ such that corticogenesis at E18.5 was at a more advanced stage. This modulation of glial maturation did not alter neuronal numbers at the end of corticogenesis but perturbation of subtle aspects of the neuronal progeny remains a possibility.

Based on the observation that there are fewer neuronal nuclei at P5, but not at E18.5 (the end of neurogenesis), chronic morphine exposure and opioid signalling could also modulate apoptosis that occurs within the neonatal cerebral cortex. Opioid effects on neuronal apoptosis are supported by observations in the literature that have shown chronic morphine induced apoptosis in the adult brain (Yin et al., 1999; Emeterio et al., 2006). Apoptosis was not measured directly in the present study.

Two major conclusions can be drawn from this thesis. 1) Support is given for the hypothesis that opioid receptors (present within the proliferating neuroepithelia of the developing forebrain) do not directly modulate proliferation. Rather, opioids lead to subtle perturbations in the intrinsic neurogenic programme of cortical progenitor cells. 2) The effects of opiate exposure on development of the cerebral cortex may be delineated into signalling that affects the prenatal histogenesis of the cerebral cortex, as well as the apoptotic remodelling of the cerebral cortex that occurs postnatally. Evidence for increased 
apoptosis from the present study comes from the loss of neurons in the morphine-exposed P5 cerebral cortex, despite no differences in neuronal density in the E18.5 foetus (Chapter 6). Thus, through development, it is possible that opioid receptors have different roles at different times. The possibility of multiple roles for opioid receptors during development of the brain mirrors the versatile nature of opioid receptors in modulating the wider physiological environment of the organism through independent actions on multiple organ systems.

7.5 What is the Significance of Opioid Signalling in Development of the CNS?

As well as providing evidence that the opioid system can modulate development of the cerebral cortex in the mouse, the finding that opioid receptors are expressed on progenitor cells within the human foetal brain (Chapter 4) makes the study relevant to human brain development in the face of opioid exposure during pregnancy. This significance is twofold.

\subsubsection{A Biological Role for the Endogenous Opioid System in Corticogenesis?}

The opioid system has long been implicated in cellular proliferation, especially in vitro, although some studies have provided strong evidence that opioids also modulate proliferation in vivo (Miller et al., 1982; Kornblum et al., 1987; Schmahl et al., 1989; Lorber et al., 1990; Bartolome et al., 1991; Stiene-Martin et al., 2001). However, our study is the first to demonstrate a clear set of effects of morphine on corticogenesis of the prenatal 
mouse. This is important in the first instance since it provides evidence that the opioid system forms a part of the complex and dynamic system that is the developing cerebral cortex. Owing to the observed slowing in cell cycle, it appears that in the developing cortex, the holistic role for the opioid system would likely be growth limiting in nature. Although the opioid system may modulate normal development of the cerebral cortex, the extent to which it does this remains unclear, but it is likely that it only plays a minor role. This is evident from the normal brain morphology of the triple opioid receptor $\mathrm{KO}$ mice (Gaveriaux-Ruff and Kieffer, 2002); thus, either there must be some system redundancy, i.e., other systems that replace opioid signalling or it indicates that opioids are not active during normal development (see Chapter 1). In line with this, only minor developmental abnormalities are seen in humans prenatally exposed to opiates (Kaltenbach, 1994; Lester et al., 2002). The opioid system presumably comprises one of the many signalling systems that add complexity, control, and fine tuning to the development of the cerebral cortex.

\subsubsection{A Highly Utilised Drug with Lasting Consequences?}

Since the opioid system is implicated in cortical development, exogenous opiate drugs may interfere with this development. There are two ways in which humans may be exposed to opiates prenatally: exposure from opiates as a course of maternal analgesia and exposure from maternal opiate abuse. Maternal opiate abuse may also be further divided into uncontrolled opiate abuse or controlled maintenance programs that often employ opiates such as methadone or buprenorphine (Lejeune et al., 2006). Although large studies have found no major opiate-induced impacts on measures such as IQ and motor performance in 
humans (Kaltenbach, 1994; Ornoy et al., 1996; Hans and Jeremy, 2001; Lester et al., 2002), it cannot be ruled out that opiate exposure may subtly change aspects of human cognition, especially given the evidence in this thesis that opiate exposure during corticogenesis alters cellular behaviour and development.

As discussed in Chapter 6, changes that occur early in life might not persist into adulthood. Even if these changes persist into adulthood, they may not actually result in behavioural abnormalities. The human cerebral cortex is a robust organ that displays substantial plasticity (Glazewski and Fox, 1996) and has the ability to restore cognitive function via astonishing compensatory changes after damage from stroke or mechanical injury (Nudo, 2006). Answering the question of whether opiate exposure causes long lasting changes in human behaviour or intellect would require further research on adult human subjects. Even though prenatal opiate exposure in humans does not elicit major developmental defects, the fact the opioid system has been implicated in development of the cerebral cortex means further analysis of human subjects prenatally exposed to opiates is warranted. However, this may be somewhat of a flippant suggestion. In an overview of research from the human maternal opiate abuse field, Kaltenbach (1994) called for studies on the developmental effects of opiate abuse in humans to abandon multifactorial research paradigms that try to control for confounding factors such as socio-economic status and poly-drug abuse. Instead it was suggested that the best way to move forward in our understanding of how opiates affect human development is to include opiate abuse into the context of a 'multi-effects model' in which opiate exposure is considered within a cumulative framework of risk 
factors that stem from maternal drug abuse and addiction. Animal and subsequent human biological research would therefore provide the basis of a part of this framework. 


\section{Appendix}

\subsection{Appendix 1 -Reagent List}

- $10 \times$ phosphate buffered saline (PBS)
○ $\mathrm{NaCl}$
$80 \mathrm{~g}$
- $\mathrm{Na}_{2} \mathrm{HPO}_{4}$ (Anhydrous)
$14.2 \mathrm{~g}$
○ $\mathrm{KCl}$
$2 \mathrm{~g}$
○ $\mathrm{KH}_{2} \mathrm{PO}_{4}$
$2.4 \mathrm{~g}$

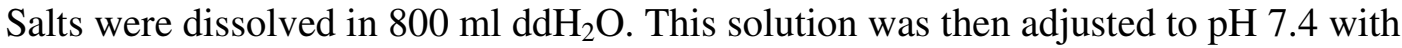
HCL. The solution was then adjusted to 11 . 10xPBS was split into $500 \mathrm{ml}$ aliquots and autoclaved. 1xPBS $(0.15 \mathrm{M})$ was prepared from this stock.

- $4 \%$ paraformaldehyde (PFA)
○ Paraformaldehyde
$40 \mathrm{~g}$
$\circ 1 \times$ PBS
$960 \mathrm{ml}$

PFA was made up to a volume of $800 \mathrm{ml}$ with $1 \mathrm{x}$ PBS. This solution was adjusted to $\mathrm{pH}$ 12 with $\mathrm{NaOH}$. Once the PFA had dissolved, the solution was adjusted to $\mathrm{pH} 7.4$ and made up to 11 with $1 \times$ PBS.

- DAB solution $(0.5 \mathrm{mg} / \mathrm{ml})$
○ DAB
$5 \mathrm{mg}$ 

$\circ 1 \times \mathrm{PBS}$
$10 \mathrm{ml}$
○ $30 \% \mathrm{H}_{2} \mathrm{O}_{2}$
$10 \mu 1$

Immediately prior to use, DAB was added to PBS and vortexed. This left a white precipitate at the bottom of the tube that did not dissolve. $\mathrm{H}_{2} \mathrm{O}_{2}$ was then added and this solution was directly applied to histological sections to reveal HRP conjugates.

- DNAse I buffer for BrdU epitope retrieval
$\circ$ Tris
$4.85 \mathrm{~g}$
○ $\mathrm{CaCl}_{2} \cdot 2 \mathrm{H}_{2} \mathrm{O}$
$1.47 \mathrm{~g}$
○ $\mathrm{MgCl}_{2} \cdot 6 \mathrm{H}_{2} \mathrm{O}$
$1.22 \mathrm{~g}$
○ $\mathrm{NaCl}$
$0.58 \mathrm{~g}$

Mix constituents in 11 of $\mathrm{ddH}_{2} \mathrm{O}$.

- $50 \times$ Tris-Acetate-EDTA (TAE buffer)
○ Tris
$242 \mathrm{~g}$
$\circ \quad 0.5 \mathrm{M} \mathrm{Na}_{2} \operatorname{EDTA}(\mathrm{aq})$
$100 \mathrm{ml}$
- Glacial acetic acid
$57.1 \mathrm{ml}$

Mix constituents and make up to 11 with $\mathrm{ddH}_{2} \mathrm{O}$.

- 6x PCR product loading buffer
- Glycerol
$3 \mathrm{ml}$
○ $\mathrm{H}_{2} \mathrm{O}$
$7 \mathrm{ml}$
- Bromophenol blue
A few crystals to colour 
Mix constituents and store at $4{ }^{\circ} \mathrm{C}$.

- RIPA buffer
○ Tris
$606 \mathrm{mg}$
$\circ \mathrm{NaCl}$
$877 \mathrm{mg}$
- $\mathrm{Na}_{2}$ EDTA
$37.22 \mathrm{mg}$
○ Triton X-100
$1 \mathrm{ml}$
- Sodium Deoxycholate
$1 \mathrm{~g}$
- SDS
$100 \mathrm{mg}$

Mix constituents and make up to $100 \mathrm{ml}$ with $\mathrm{ddH}_{2} \mathrm{O}$

- Western transfer buffer
$\circ$ Tris
$3.03 \mathrm{~g}$
○ SDS
$1 \mathrm{~g}$
○ Glycine
$14.4 \mathrm{~g}$
○ Methanol
$200 \mathrm{ml}$

These components were mixed and adjusted to a volume of 11 with $\mathrm{ddH}_{2} \mathrm{O}$.

- Thionin Nissl stain

- Stock thionin stain $(1.3 \%)$

- Thionin $13 \mathrm{~g}$

- $\mathrm{ddH}_{2} \mathrm{O}$ 
Thionin was stirred and heated for $1 \mathrm{~h}$. Thionin solution was then filtered.

$\circ$ Working thionin solution $(0.2 \%, \mathrm{pH} 4)$

- 1 M Acetic acid $\quad 80 \mathrm{ml}$

- $1 \mathrm{M} \mathrm{NaOH} \quad 14.4 \mathrm{ml}$

- $1.3 \%$ thionin stock $\quad 76.4 \mathrm{ml}$

These constituents were mixed and diluted to $400 \mathrm{ml}$ with $\mathrm{ddH}_{2} \mathrm{O}$. 
- Deparaffinise and rehydrate paraffin embedded sections of brain:

○ $100 \%$ xylene for 10 mins x 2

○ $100 \%$ ethanol for 2 mins

- $90 \%$ ethanol for 2 mins

○ $80 \%$ ethanol for 2 mins

○ $70 \%$ ethanol for 2 mins

- Wash in $\mathrm{ddH}_{2} \mathrm{O}$ for 2 mins

O NOTE: As phosphate salts cause thionin to precipitate, PBS must not be used for this procedure. This causes purple precipitate to collect on the tissue sections.

- Stain in $0.2 \%$ thionin until desired colouring is obtained

- This typically took 3-4 mins for procedures performed in this thesis research

- Wash slides in $\mathrm{ddH}_{2} \mathrm{O}$ for 2 mins

- Dehydrate and clear sections:

○ $70 \%$ ethanol for 2 mins

○ $80 \%$ ethanol for 2 mins

○ $90 \%$ ethanol for 2 mins

○ $100 \%$ ethanol for 2 mins

○ $100 \%$ xylene for 10 mins x 2

- Remove excess xylene and mount sections in DPX mounting media 
- Factors that decreased Nissl staining intensity:

$\circ$ Ethanol used in the dehydration step usually leeched thionin from the stained brain sections. To account for this, sections were usually initially over-stained in thionin.

- Nissl staining intensity was also markedly decreased by antigen recovery in boiling sodium citrate. Nissl staining sections that had been stained for BrdU therefore required much more time to stain in thionin. 


\subsection{Appendix 3 - Optimisation of BrdU Staining on Frozen Tissue Sections}

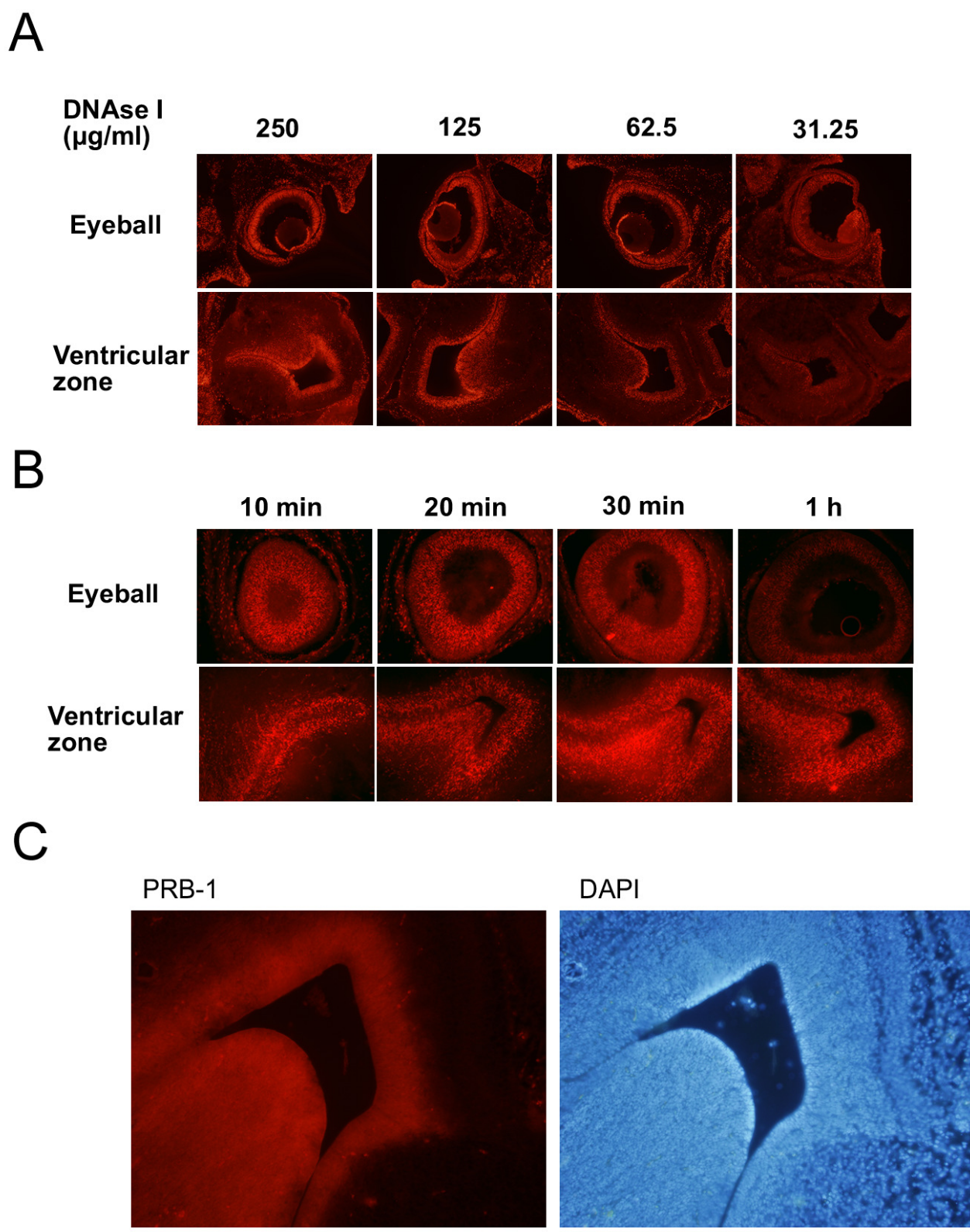

Fig. 8.1. Optimisation of BrdU staining of fixed-frozen tissue. Optimisation of fluorescence staining for BrdU incorporation in frozen sections of tissue required an antigen retrieval technique that was not as harsh as using boiling sodium citrate buffer or 1-2N HCL, as is used in some studies. Epitope retrieval using DNAse I was chosen as it did not destroy the frozen tissue sections. Panel A shows a titration in DNAse I concentration 
from $250-31.25 \mu \mathrm{g} / \mathrm{ml}$. From these results, a concentration of $250 \mu \mathrm{g} / \mathrm{ml}$ was chosen for further DNAse I retrieval optimisation. Panel B shows optimisation of DNAse I digestion time. A digestion time of 10 mins was chosen for future experiments. Interestingly, over-digestion is shown in the neuroepithelium of the eyeball at $1 \mathrm{~h}$. Panel $\mathrm{C}$ shows diffusion of the anti-BrdU antibody, PRB-1. BrdU staining (red fluorescence) can be seen around the lateral ventricle of the E15.5 brain. However, staining is diffuse and has spread out from its normal localisation within the VZ/SVZ. This phenomena was finally resolved by performing a postfixation step in 4\% PFA for 5 mins after incubation with the anti-BrdU antibody. 


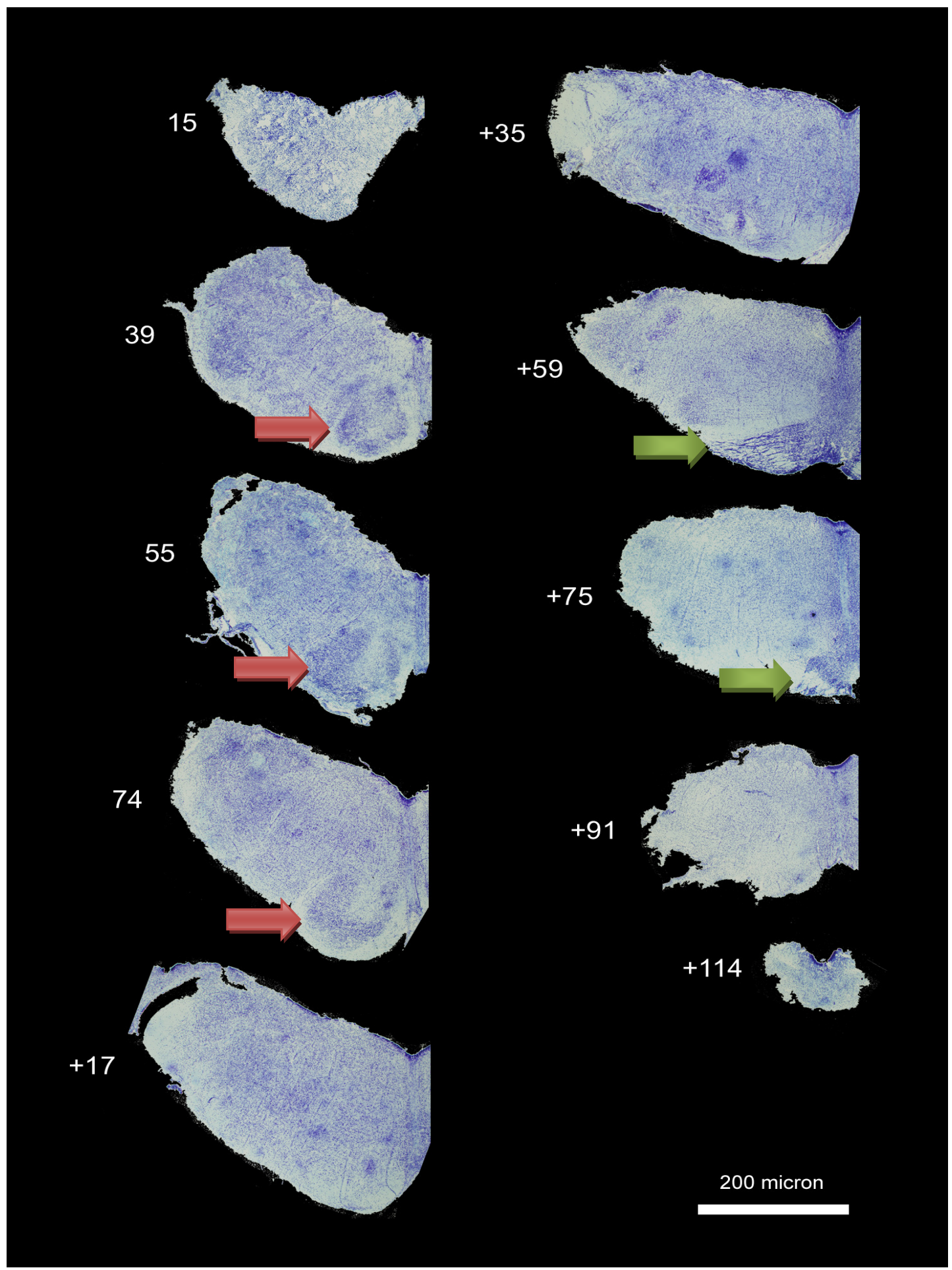


Fig. 8.2. Sectioning and staining of Brain 1. Brain $1(12 \mathrm{GW})$ was sectioned at $40 \mu \mathrm{m}$ thickness and Nissl stained in order to conclusively identify its anatomy. Brain 1 comprised entirely of brainstem, as shown by these Nissl stained sections. Regions of note are the inferior olivary nucleus on sections 39, 55 and 74 (red arrows), and the pons, on sections +59 and +75 (green arrows). 

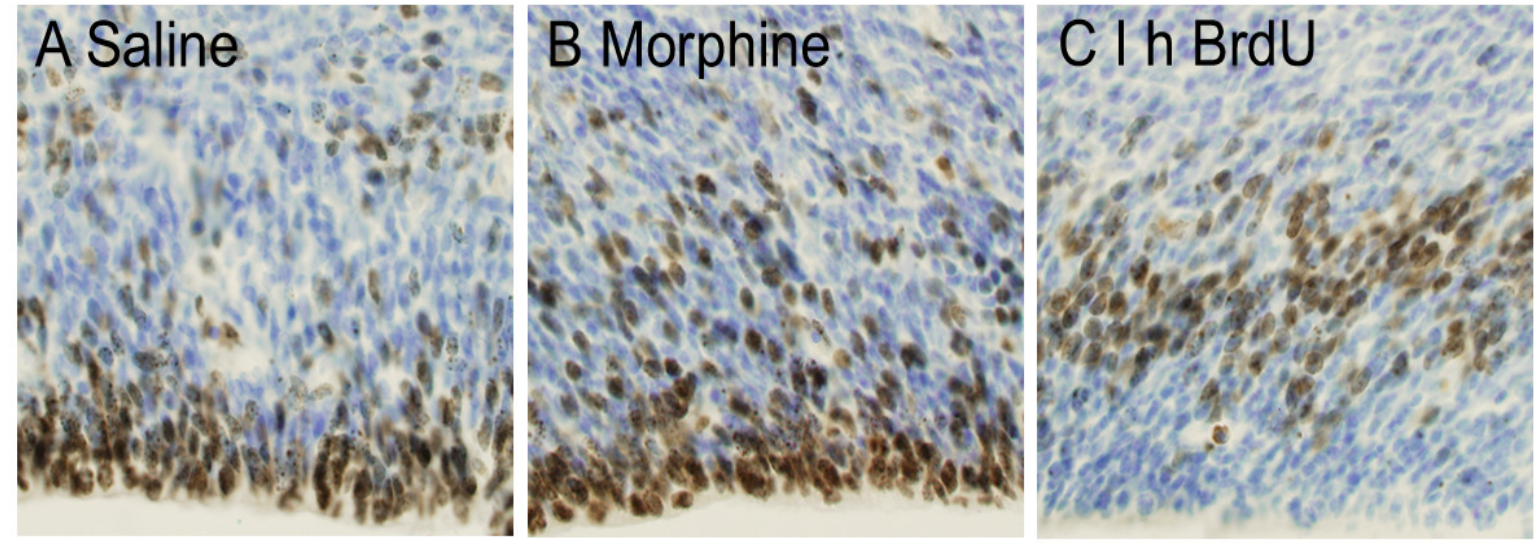

Fig. 8.3. The morphine-INM pilot experiment. After establishing that there was no effect of morphine on BrdU labelling (Chapter 5, Fig. 4, panel A), it was decided to investigate whether or not morphine had any effect on INM to the ventricular surface $\left(\mathrm{G}_{2}\right.$ phase). This preliminary experiment was performed to assess whether or not this idea was viable before committing large numbers of pregnant dams to full experiments. Panel A is from an E15 saline-treated control. It shows the VZ which is DAB-stained for BrdU that was injected $6 \mathrm{~h}$ prior to sacrifice. This same panel showed that nearly all cells had migrated to the ventricular surface. Panel B shows that acute morphine treatment resulted in an accumulation of BrdU-labelled nuclei in the basal regions of the VZ (the same drug administration regime was used as the one used to assess the effect of morphine exposure on INM in Chapter 5). Panel C shows VZ DAB-stained for BrdU that was injected $1 \mathrm{~h}$ prior to sacrifice as a comparison for INM to the ventricular surface. After assessing these results, it was decided to plan experiments that would investigate the effects of morphine on INM and $\mathrm{G}_{2} / \mathrm{M}_{\text {phase duration. }}$ Animals used to generate the pictures presented here were not used in the final analysis of these experiments. 
8.6 Appendix 6 - Calculation of Likely Concentration of Peak Plasma Morphine

Morphine used in this thesis research was morphine sulfate $\left(\mathrm{C}_{34} \mathrm{H}_{40} \mathrm{~N}_{2} \mathrm{O}_{10} \mathrm{~S} \cdot 5 \mathrm{H} 2 \mathrm{O}, \mathrm{MW}=\right.$ 758.83).

We used a dose of $10 \mathrm{mg} / \mathrm{kg}$ morphine sulfate. That is:

$0.01 \mathrm{~g} / \mathrm{kg} / 758.83 \mathrm{~g} / \mathrm{mol}=1.32 \times 10^{-5} \mathrm{~mol} / \mathrm{kg}$

or

$13.2 \mu \mathrm{mol} / \mathrm{kg}$ of morphine sulfate

As there are two molecules of morphine in one mole of morphine sulfate, this means that $10 \mathrm{mg} / \mathrm{kg}$ of morphine sulfate is $26.4 \mu \mathrm{mol} / \mathrm{kg}$ of morphine. As Handal et al. (2002) used a dose of $80 \mu \mathrm{mol} / \mathrm{kg}$ of morphine hydrochloride in their studies, the dose of morphine used in the present research is

$100 \times(26.4 \mu \mathrm{mol} / \mathrm{kg} / 80 \mu \mathrm{mol} / \mathrm{kg})=33 \%$

of the dose of morphine administered to animals in the pharmacokinetic studies by Handal et al. (2002) who showed that a dose of $80 \mu \mathrm{mol} / \mathrm{kg}$ produced a peak plasma concentration of $18 \mu \mathrm{M}$. Therefore, our dose of $10 \mathrm{mg} / \mathrm{kg}$ is likely to produce a peak plasma concentration of

$18 \mu \mathrm{M} \times 0.33=5.9 \mu \mathrm{M}$ 
MTT Assay

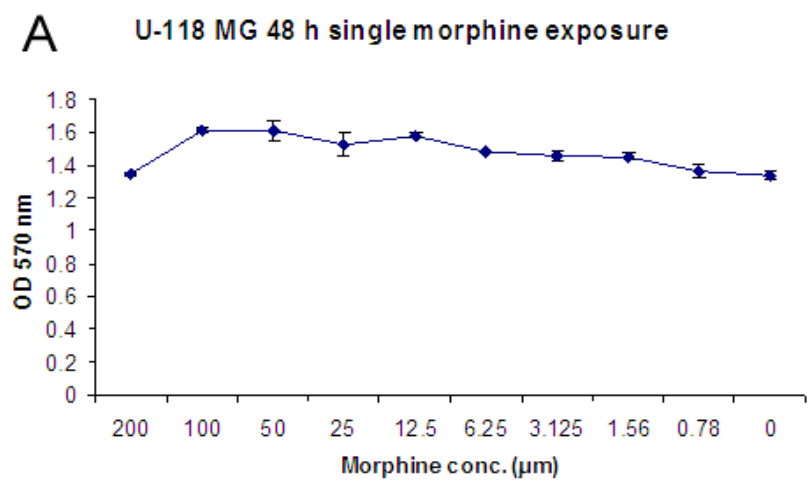

B SH-SY5Y $48 \mathrm{~h}$ single morphine exposure

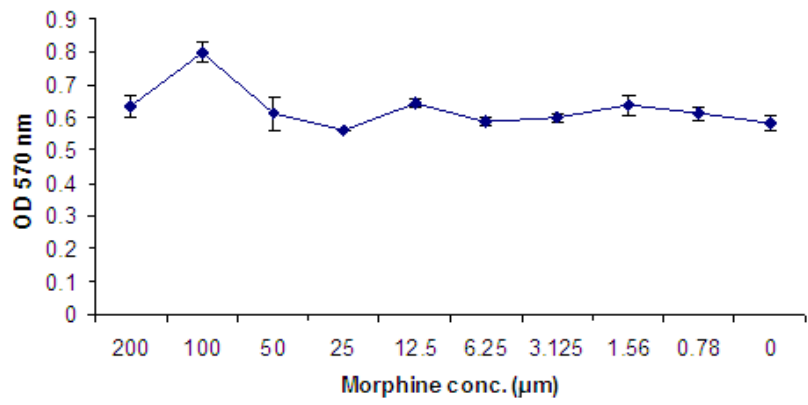

C N2A 48 h single morphine exposure

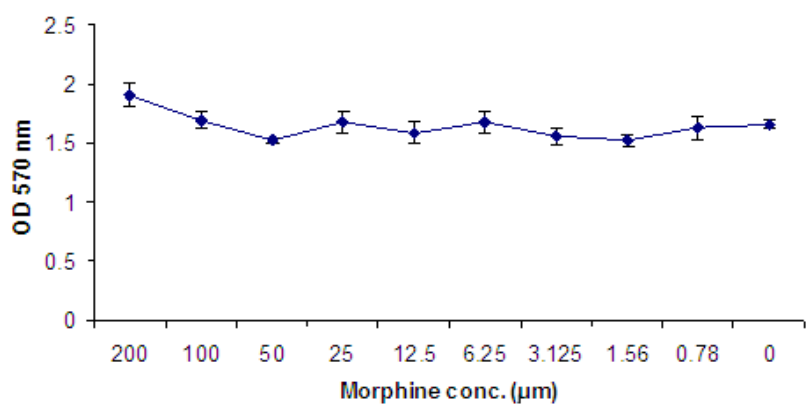

Fig. 8.4. Morphine and growth of cell cultures. MTT assay was used as an indirect measure of proliferation on cell culture lines. The MTT assay uses a yellow tetrazolium salt that is reduced to purple formazan at the mitochondria to assay for respiration. Cells were cultured in RPMI media (Invitrogen, 11875-085) containing $10 \%$ foetal bovine serum and $1 \%$ penicillin/streptomycin. Before the effects of morphine were tested on the 
growth of three different cell lines (U-118 MG (human glioblastoma), SH-SY5Y (human neuroblastoma) and N2A (mouse neuroblastoma), the concentration of cells used in this assay was calibrated such that there was no ceiling effect. To determine whether morphine inhibited proliferation of these cell lines, cells were cultured in the presence of a range of concentrations of morphine (x-axis). It was shown that after $48 \mathrm{~h}$ of exposure to these concentrations of morphine, that there was no effect whatsoever on cellular proliferation as measured by MTT assay. All data points are the average of 3 replicates. Values are the mean \pm SEM. 


\section{Reference List}

Abbadie, C., Pan, Y.X., Drake, C.T., Pasternak, G.W., 2000. Comparative immunohistochemical distributions of carboxy terminus epitopes from the muopioid receptor splice variants MOR-1D, MOR-1 and MOR-1C in the mouse and rat CNS. Neuroscience 100, 141-153.

Abbadie, C., Pan, Y.X., Pasternak, G.W., 2004. Immunohistochemical study of the expression of exon 11-containing mu opioid receptor variants in mouse brain. Neuroscience 127, 419-430.

Agasse, F., Benzakour, O., Berjeaud, J.M., Roger, M., Coronas, V., 2006. Endogenous factors derived from embryonic cortex regulate proliferation and neuronal differentiation of postnatal subventricular zone cell cultures. Eur. J. Neurosci. 23, 1970-1976.

Ajioka, I., Maeda, T., Nakajima, K., 2006. Identification of ventricular-side enriched molecules regulated in a stage-dependent manner during cerebral cortical development. Eur. J. Neurosci. 23, 296-308.

Angelogianni, P., Li, H.L., Gianoulakis, C., 2000. Ontogenesis of proopiomelanocortin and its processing to beta-endorphin by the fetal and neonatal rat brain. Neuroendocrinology 72, 231-241.

Angevine, J.B. Jr, Sidman, R.L., 1961. Autoradiographic study of cell migration during histogenesis of cerebral cortex in the mouse. Nature 192, 766-768.

Anthony, T.E., Klein, C., Fishell, G., Heintz, N., 2004. Radial glia serve as neuronal progenitors in all regions of the central nervous system. Neuron 41, 881-890.

Arden, J.R., Segredo, V., Wang, Z., Lameh, J., Sadee, W., 1995. Phosphorylation and agonist-specific intracellular trafficking of an epitope-tagged mu-opioid receptor expressed in HEK 293 cells. J. Neurochem. 65, 1636-1645.

Bai, J., Ramos, R.L., Ackman, J.B., Thomas, A.M., Lee, R.V., LoTurco, J.J., 2003. RNAi reveals doublecortin is required for radial migration in rat neocortex. Nature Neurosci. 6, 1277-1283.

Bare, L.A., Bansson, E., Yang, D., 1994. Expression of two variants of the human mu opioid receptor mRNA in SK-N-SH cells and human brain. FEBS Lett. 354, 213216.

Barg. J., Simantov, R., 1989. Developmental profile of kappa, mu and delta opioid receptors in the rat and guinea pig cerebellum. Dev. Neurosci. 11, 428-434.

Barg, J., Belcheva, M., McHale, R., Levy, R., Vogel, Z., Coscia, C.J., 1993. Betaendorphin is a potent inhibitor of thymidine incorporation into DNA via mu- and kappa-opioid receptors in fetal rat brain cell aggregates in culture. J. Neurochem. 60, 765-767.

Barr, G.A., Zadina, J.E., 1999. The ontogeny of endomorphin-1- and endomorphin-2-like immunoreactivity in rat brain and spinal cord. Ann. N. Y. Acad. Sci. 897, 145-153. 
Barry, D., McDermott, K., 2005. Differentiation of radial glia from radial precursor cells and transformation into astrocytes in the developing rat spinal cord. Glia 50, 187197.

Bartolome, J.V., Bartolome, M.B., Lorber, B.A., Dileo, S.J., Schanberg, S.M., 1991. Effects of central administration of beta-endorphin on brain and liver DNA synthesis in preweanling rats. Neuroscience 40, 289-294.

Bartolome, J.V., Lorber, B.A., Bartolome, M.B., 1994. Brain cholecystokinin and betaendorphin systems may antagonistically interact to regulate tissue DNA synthesis in rat pups. Brain Res. 661, 19-24.

Baye, L.M., Link, B.A., 2007a. Interkinetic nuclear migration and the selection of neurogenic cell divisions during vertebrate retinogenesis. J. Neurosci. 27, 1014310152.

Baye, L.M., Link, B.A., 2007b. Nuclear migration during retinal development. Brain Res. Epub ahead of print.

Belcheva, M.M., Vogel, Z., Ignatova, E., Avidor-Reiss, T., Zippel, R., Levy, R., Young, E.C., Barg, J., Coscia, C.J., 1998. Opioid modulation of extracellular signalregulated protein kinase activity is ras-dependent and involves Gbetagamma subunits. J. Neurochem. 70, 635-645.

Belcheva, M.M., Wong, Y.H., Coscia, C.J., 2000. Evidence for transduction of mu but not kappa opioid modulation of extracellular signal-regulated kinase activity by G(z) and G(12) proteins. Cell Signal. 12, 481-489.

Belcheva, M.M., Haas, P.D., Tan, Y., Heaton, V.M., Coscia, C.J., 2002. The fibroblast growth factor receptor is at the site of convergence between mu-opioid receptor and growth factor signaling pathways in rat C6 glioma cells. J. Pharmacol. Exp. Ther. 303, 909-918.

Belcheva, M.M., Tan, Y., Heaton, V.M., Clark, A.L., Coscia, C.J., 2003. Mu opioid transactivation and down-regulation of the epidermal growth factor receptor in astrocytes: implications for mitogen-activated protein kinase signaling. Mol. Pharmacol. 64, 1391-1401.

Belcheva, M.M., Clark, A.L., Haas, P.D., Serna, J.S., Hahn, J.W., Kiss, A., Coscia, C.J., 2005. Mu and kappa opioid receptors activate ERK/MAPK via different protein kinase $\mathrm{C}$ isoforms and secondary messengers in astrocytes. J. Biol. Chem. 280, 27662-27669.

Beloozerova, I.N., Sirota, M.G., Swadlow, H.A., 2003. Activity of different classes of neurons of the motor cortex during locomotion. J. Neurosci. 23, 1087-1097.

Bezard, E., Gross, C.E., Brotchie, J.M., 2003. Presymptomatic compensation in Parkinson's disease is not dopamine-mediated. Trends Neurosci. 26, 215-221.

Bilsky, E.J., Bernstein, R.N., Hruby, V.J., Rothman, R.B., Lai, J., Porreca, F., 1996. Characterization of antinociception to opioid receptor selective agonists after antisense oligodeoxynucleotide-mediated "knock-down" of opioid receptor in vivo. J. Pharmacol. Exp. Ther. 277, 491-501.

Bohn, L.M., Belcheva, M.M., Coscia, C.J., 2000a. Mu-opioid agonist inhibition of kappaopioid receptor-stimulated extracellular signal-regulated kinase phosphorylation is dynamin-dependent in C6 glioma cells. J. Neurochem. 74, 574-581.

Bohn, L.M., Belcheva, M.M., Coscia, C.J., 2000b. Mitogenic signaling via endogenous kappa-opioid receptors in C6 glioma cells: evidence for the involvement of protein 
kinase $\mathrm{C}$ and the mitogen-activated protein kinase signaling cascade. J. Neurochem. 74, 564-573.

Bohn, L.M., Raehal, K.M., 2006. Opioid receptor signalling: relevance for gastrointestinal therapy. Curr. Opin. Pharmacol. 6, 559-563.

Bolan, E.A., Tallarida, R.J., Pasternak, G.W., 2002. Synergy between mu opioid ligands: evidence for functional interactions among mu opioid receptor subtypes. J. Pharmacol. Exp. Ther. 303, 557-562.

Bolan, E.A., Pan, Y.X., Pasternak, G.W., 2004. Functional analysis of MOR-1 splice variants of the mouse mu opioid receptor gene Oprm. Synapse 51, 11-18.

Bonthius, D.J., McKim, R., Koele, L., Harb, H., Karacay, B., Mahoney, J., Pantazis, N.J., 2004. Use of frozen sections to determine neuronal number in the murine hippocampus and neocortex using the optical disector and optical fractionator. Brain Res. Brain Res. Protoc. 14, 45-57.

Boulder Committee: Angevine, J.B. Jr, Bodian, D., Coulombre, A.J., Edds, M.V. Jr, Hamburger, V., Jacobson, M., Lyser, K.M., Prestige, M.C., Sidman, R.L., Varon, S., Weiss, P.A., 1970. Embryonic vertebrate central nervous system: revised terminology. The Boulder Committee. Anat. Rec. 166, 257-261.

Brazel, C.Y., Romanko, M.J., Rothstein, R.P., Levison, S.W., 2003. Roles of the mammalian subventricular zone in brain development. Prog. Neurobiol. 69, 49-69.

Brisson, M., Tan, L., Park, R., Hamby, K., 2000. Identification of nonspecific products using melt-curve analysis. Bio-Rad Laboratories, Inc. Technical note 2684.

Brownstein, M.J., 1993. A brief history of opiates, opioid peptides, and opioid receptors. Proc. Natl. Acad. Sci. U.S.A. 90, 5391-5393.

Buchenauer, D., Turnbow, M., Peters, M.A., 1974. Effect of chronic methadone administration on pregnant rats and their offspring. J. Pharmacol. Exp. Ther. 189, 66-71.

Bulfone, A., Puelles, L., Porteus, M.H., Frohman, M.A., Martin, G.R., Rubenstein, J.L., 1993. Spatially restricted expression of Dlx-1, Dlx-2 (Tes-1), Gbx-2, and Wnt-3 in the embryonic day 12.5 mouse forebrain defines potential transverse and longitudinal segmental boundaries. J. Neurosci. 13, 3155-3172.

Bullwinkel, J., Baron-Lühr, B., Lüdemann, A., Wohlenberg, C., Gerdes, J., Scholzen, T., 2006. Ki-67 protein is associated with ribosomal RNA transcription in quiescent and proliferating cells. J. Cell. Physiol. 206, 624-635.

Bunikowski, R., Grimmer, I., Heiser, A., Metze, B., Schafer, A., Obladen, M., 1998. Neurodevelopmental outcome after prenatal exposure to opiates. Eur. J. Pediatr. 157, 724-730.

Burns, K. A., Ayoub, A. E., Breunig, J. J., Adhami, F., Weng, W. L., Colbert, M. C., Rakic, P., Kuan, C. Y., 2007. Nestin-CreER mice reveal DNA synthesis by nonapoptotic neurons following cerebral ischemia hypoxia. Cereb. Cortex 17, 2585-2592.

Calegari, F., Huttner, W.B., 2003. An inhibition of cyclin-dependent kinases that lengthens, but does not arrest, neuroepithelial cell cycle induces premature neurogenesis. J. Cell Sci. 116, 4947-4955.

Calegari, F., Haubensak, W., Haffner, C., Huttner, W.B., 2005. Selective lengthening of the cell cycle in the neurogenic subpopulation of neural progenitor cells during mouse brain development. J. Neurosci. 25, 6533-6538. 
Cappello, S., Attardo, A., Wu, X., Iwasato, T., Itohara, S., Wilsch-Brauninger, M., Eilken, H.M., Rieger, M.A., Schroeder, T.T., Huttner, W.B., Brakebusch, C., Gotz, M., 2006. The Rho-GTPase cdc42 regulates neural progenitor fate at the apical surface. Nat. Neurosci. 9, 1099-1107.

Carney, R.S., Bystron, I., López-Bendito, G., Molnár, Z., 2007. Comparative analysis of extra-ventricular mitoses at early stages of cortical development in rat and human. Brain Struct. Funct. 212, 37-54.

Cattaneo, E., McKay, R., 1990. Proliferation and differentiation of neuronal stem cells regulated by nerve growth factor. Nature 347, 762-765.

Caviness, V.S. Jr, Takahashi, T., Nowakowski, R.S., 1999. The G1 restriction point as critical regulator of neocortical neuronogenesis. Neurochem. Res. 24, 497-506.

Caviness, V.S. Jr, Goto, T., Tarui, T., Takahashi, T., Bhide, P.G., Nowakowski, R.S., 2003. Cell output, cell cycle duration and neuronal specification: a model of integrated mechanisms of the neocortical proliferative process. Cereb. Cortex 13, 592-598.

Chalecka-Franaszek, E., Weems, H.B., Crowder, A.T., Cox, B.M., Côté, T.E., 2000. Immunoprecipitation of high-affinity, guanine nucleotide-sensitive, solubilized muopioid receptors from rat brain: coimmunoprecipitation of the $\mathrm{G}$ proteins $\mathrm{G}$ (alpha o), G(alpha i1), and G(alpha i3). J. Neurochem. 74, 1068-1078.

Chandler, J.M., Robie, P.W., Schoolar, J.C., Desmond, M.M., 1975. The effects of methadone on maternal-fetal interactions in the rat. J. Pharmacol. Exp. Ther. 192, 549-554.

Cheema, Z.F., Wade, S.B., Sata, M., Walsh, K., Sohrabji, F., Miranda, R.C., 1999. Fas/Apo [apoptosis]-1 and associated proteins in the differentiating cerebral cortex: induction of caspase-dependent cell death and activation of NF-kappaB. J. Neurosci. 19, 1754-1770.

Chen, Y., Mestek, A., Liu, J. Yu, L., 1993a. Molecular cloning of a rat kappa opioid receptor reveals sequence similarities to the mu and delta opioid receptors. Biochem. J. 295, 625-628.

Chen, Y., Mestek, A., Liu, J., Hurley, J.A., Yu, L., 1993b. Molecular cloning and functional expression of a mu-opioid receptor from rat brain. Mol. Pharmacol. 44, 8-12.

Cheng, F., Zagon, I.S., Verderame, M.F., McLaughlin, P.J., 2007. The opioid growth factor (OGF)-OGF receptor axis uses the p16 pathway to inhibit head and neck cancer. Cancer Res. 67, 10511-10518.

Cheng, F., McLaughlin, P.J., Verderame, M.F., Zagon, I.S., 2008. The OGF-OGFR axis utilizes the p21 pathway to restrict progression of human pancreatic cancer. Mol. Cancer 7, 5.

Chenn, A., Walsh, C.A., 2002. Regulation of cerebral cortical size by control of cell cycle exit in neural precursors. Science 297, 365-369.

Christie, M.J., 2008. Cellular neuroadaptations to chronic opioids: tolerance, withdrawal and addiction. Br. J. Pharmacol. 154, 384-396.

Christoffers, K.H., Li, H., Keenan, S.M., Howells, R.D., 2003. Purification and mass spectrometric analysis of the mu opioid receptor. Brain Res. Mol. Brain Res. 118, 119-131. 
Chu, J., Zheng, H., Loh, H.H., Law, P.Y., 2008. Morphine-induced mu-opioid receptor rapid desensitization is independent of receptor phosphorylation and beta-arrestins. Cell. Signal. 20, 1616-1624.

Clark, J.A., Houghten, R., Pasternak, G.W., 1988. Opiate binding in calf thalamic membranes: a selective mu 1 binding assay. Mol. Pharmacol. 34, 308-317.

Clark, J.A., Liu, L., Price, M., Hersh, B., Edelson, M., Pasternak, G.W., 1989. Kappa opiate receptor multiplicity: evidence for two U50,488-sensitive kappa 1 subtypes and a novel kappa 3 subtype. J. Pharmacol. Exp. Ther. 251, 461-468.

Clendeninn, N.J., Petraitis, M., Simon, E.J., 1976. Ontological development of opiate receptors in rodent brain. Brain Res. 118, 157-160.

Colman, A.S., Miller, J.H., 2001. Modulation of breathing by mu1 and mu2 opioid receptor stimulation in neonatal and adult rats. Respir. Physiol. 127, 157-172.

Connor, M., Christie, M.D., 1999. Opioid receptor signalling mechanisms. Clin. Exp. Pharmacol. Physiol. 26, 493-499.

Connor, M., Osborne, P.B., Christie, M.J., 2004. Mu-opioid receptor desensitization: is morphine different? Br. J. Pharmacol. 143, 685-696.

Connor, M., Kitchen, I., 2006. Has the sun set on kappa3-opioid receptors? Br. J. Pharmacol. 147, 349-350.

Corbo, J.C., Deuel, T.A., Long, J.M., LaPorte, P., Tsai, E., Wynshaw-Boris, A., Walsh, C.A., 2002. Doublecortin is required in mice for lamination of the hippocampus but not the neocortex. J. Neurosci. 22, 7548-7557.

Corwin, M.J., Lester, B.M., Golub, H.L., 1996. The infant cry: what can it tell us? Curr. Probl. Pediatr. 26, 325-334.

Cox, J., Jackson, A.P., Bond, J., Woods, C.G., 2006. What primary microcephaly can tell us about brain growth. Trends Mol. Med. 12, 358-366.

Coyle, J.T., Pert, C.B., 1976. Ontogenic development of [3H]naloxone binding in rat brain. Neuropharmacology 15, 555-560.

Curtis, M.A., Kam, M., Nannmark, U., Anderson, M.F., Axell, M.Z., Wikkelso, C., Holtås, S., van Roon-Mom, W.M., Björk-Eriksson, T., Nordborg, C., Frisén, J., Dragunow, M., Faull, R.L., Eriksson, P.S., 2007a. Human neuroblasts migrate to the olfactory bulb via a lateral ventricular extension. Science 315, 1243-1249.

Curtis, M.A., Kam, M., Nannmark, U., Faull, R.L.M., Eriksson, P.S., 2007b. Response to comment on "Human neuroblasts migrate to the olfactory bulb via a lateral ventricular extension". Science 318, 393.

Cvejic, S., Devi, L.A., 1997. Dimerization of the delta opioid receptor: implication for a role in receptor internalization. J. Biol. Chem. 272, 26959-26964.

Dahl, J.L., Epstein, M.L., Silva, B.L., Lindberg, I., 1982. Multiple immunoreactive forms of met- and leu-enkephalin in fetal and neonatal rat brain and in rat gut. Life Sci. 31, $1853-1856$.

Davies, A.M., 2003. Regulation of neuronal survival and death by extracellular signals during development. EMBO J. 22, 2537-2545.

Davila-Garcia, M.I., Azmitia, E.C., 1989. Effects of acute and chronic administration of leu-enkephalin on cultured serotonergic neurons: evidence for opioids as inhibitory neuronal growth factors. Brain Res. Dev. Brain Res. 49, 97-103.

Daunais, J.B., Letchworth, S.R., Sim-Smelley, L.J., Smith, H.R., Childers, S.R., Porrino, L.J., 2001. Functional and anatomical localization of mu opioid receptors in the 
striatum, amygdale and extended amygdale of the nonhuman primate. J. Comp. Neurol. 433, 471-485.

Day, D.J., Mrkusich, E.M., Miller, J.H., 2007. Comparative quantitation of mRNA expression in the central nervous system using fluorescence in situ hybridization. Methods Mol. Biol. 353, 125-142.

de Carlos, J.A., López-Mascaraque, L., Valverde, F., 1996. Dynamics of cell migration from the lateral ganglionic eminence in the rat. J. Neurosci. 16, 6146-6156.

Dehay, C., Kennedy, H., 2007. Cell-cycle control and cortical development. Nat. Rev. Neurosci. 8, 438-450.

Delalle, I., Takahashi, T., Nowakowski, R.S., Tsai, L.H., Caviness, V.S. Jr., 1999. Cyclin E-p27 opposition and regulation of the G1 phase of the cell cycle in the murine neocortical PVE: a quantitative analysis of mRNA in situ hybridization. Cereb. Cortex 9, 824-832.

De Marchis, S., Fasolo, A., Puche, A.C., 2004. Subventricular zone-derived neuronal progenitors migrate into the subcortical forebrain of postnatal mice. J. Comp. Neurol. 476, 290-300.

Ding, Y.Q., Kaneko, T., Nomura, S., Mizuno, N., 1996. Immunohistochemical localization of mu-opioid receptors in the central nervous system of the rat. J. Comp. Neurol. 367, 375-402.

Doetsch, F., Caillé, I., Lim, D.A., García-Verdugo, J.M., Alvarez-Buylla, A., 1999. Subventricular zone astrocytes are neural stem cells in the adult mammalian brain. Cell 97, 703-716.

Dorus, S., Vallender, E.J., Evans, P.D., Anderson, J.R., Gilbert, S.L., Mahowald, M., Wyckoff, G.J., Malcom, C.M., Lahn, B.T., 2004. Accelerated evolution of nervous system genes in the origin of Homo sapiens. Cell 119, 1027-1040.

Dutriez, I., Sales, N., Fournie-Zaluski, M.C., Roques, B.P., 1992. Pre- and post-natal ontogeny of neutral endopeptidase 24-11 ('enkephalinase') studied by in vitro autoradiography in the rat. Experientia 48, 290-300.

Eisch, A.J., Barrot, M., Schad, C.A., Self, D.W., Nestler, E.J., 2000. Opiates inhibit neurogenesis in the adult rat hippocampus. Proc. Nat. Acad. Sci. U.S.A. 97, 75797584.

Emeterio, E.P., Tramullas, M., Hurlé, M.A., 2006. Modulation of apoptosis in the mouse brain after morphine treatments and morphine withdrawal. J. Neurosci. Res. 83, 1352-1361.

Endl, E., Steinbach, P., Knüchel, R., Hofstädter, F., 1997. Analysis of cell cycle-related Ki67 and p120 expression by flow cytometric BrdUrd-Hoechst/7AAD and immunolabeling technique. Cytometry 29, 233-241.

Eppler, C.M., Hulmes, J.D., Wang, J.B., Johnson, B., Corbett, M., Luthin, D.R., Uhl, G.R., Linden, J., 1993. Purification and partial amino acid sequence of a mu opioid receptor from rat brain. J. Biol. Chem. 268, 26447-26451.

Erdmann, G., Schutz, G., Berger, S., 2007. Inducible gene inactivation in neurons of the adult mouse forebrain. BMC Neurosci. 8(63).

Erdos, E.G., Skidgel, R.A., 1989. Neutral endopeptidase 24.11 (enkephalinase) and related regulators of peptide hormones. FASEB J. 3, 145-151.

Erspamer, V., Melchiorri, P., Falconieri-Erspamer, G., Negri, L., Corsi, R., Severini, C., Barra, D., Simmaco, M., Kreil, G., 1989. Deltorphins: a family of naturally 
occurring peptides with high affinity and selectivity for delta opioid binding sites. Proc. Natl. Acad. Sci. U.S.A. 86, 5188-5192.

Evans, C.J., Keith, D.E., Morrison, H., Magendzo, K., Edwards, R.H., 1992. Cloning of a delta opioid receptor by functional expression. Science 258, 1952-1955.

Fay, P.W., 1975. The Opium War 1840-1842. The University of North Carolina Press, U.S.A.

Fields, H., 2004. State-dependent opioid control of pain. Nat. Rev. Neurosci. 5, 565-575.

Fowler, C.B., Pogozheva, I.D., Lomize, A.L., LeVine, H., Mosberg, H.I., 2004. Complex of an active mu-opioid receptor with a cyclic peptide agonist modeled from experimental constraints. Biochemistry 43, 15796-15810.

Francis, F., Koulakoff, A., Boucher, D., Chafey, P., Schaar, B., Vinet, M.C., Friocourt, G., McDonnell, N., Reiner, O., Kahn, A., McConnell, S.K., Berwald-Netter, Y., Denoulet, P., Chelly, J., 1999. Doublecortin is a developmentally regulated, microtubule-associated protein expressed in migrating and differentiating neurons. Neuron 23, 247-256.

Frantz, G.D., McConnell, S.K., 1996. Restriction of late cerebral cortical progenitors to an upper-layer fate. Neuron 17, 55-61.

Frederiksen, K., Jat, P.S., Valtz, N., Levy, D., McKay, R., 1988. Immortalization of precursor cells from the mammalian CNS. Neuron 1, 439-448.

Frederiksen, K., McKay, R.D.G., 1988. Proliferation and differentiation of rat neuroepithelial precursor cells in vivo. J. Neurosci. 8, 1144-1151.

Gal, J.S., Morozov, Y.M., Ayoub, A.E., Chatterjee, M., Rakic, P., Haydar, T.F., 2006. Molecular and morphological heterogeneity of neural precursors in the mouse neocortical proliferative zones. J. Neurosci. 26, 1045-1056.

Ganat, Y.M., Silbereis, J., Cave, C., Ngu, H., Anderson, G.M., Ohkubo, Y., Ment, L.R., Vaccarino, F.M., 2006. Early postnatal astroglial cells produce multilineage precursors and neural stem cells in vivo. J. Neurosci. 26, 8609-8621.

Gao, W.J., Zheng, Z.H., 2004. Target-specific differences in somatodendritic morphology of layer V pyramidal neurons in rat motor cortex. J. Comp. Neurol. 476, 174-185.

Gaveriaux-Ruff, C., Kieffer, B.L., 2002. Opioid receptor genes inactivated in mice: the highlights. Neuropeptides 36, 62-71.

George, S.R., Fan, T., Xie, Z., Tse, R., Tam, V., Varghese, G., O’Dowd, B.F., 2000. Oligomerization of mu- and delta-opioid receptors. Generation of novel functional properties. J. Biol. Chem. 275, 26128-26135.

George, M.J., 2006. The site of action of epidurally administered opioids and its relevance to postoperative pain management. Anaesthesia 61, 659-664.

Georges, F., Normand, E., Bloch, B., LeMoine, C., 1998. Opioid receptor gene expression in the rat brain during ontogeny, with special reference to the mesostriatal system: an in situ hybridization study. Brain Res. Dev. Brain Res. 109, 187-199.

Gerdes, J., Lemke, H., Baisch, H., Wacker, H.H., Schwab, U., Stein, H., 1984. Cell cycle analysis of a cell proliferation-associated human nuclear antigen defined by the monoclonal antibody Ki-67. J. Immunol. 133, 1710-1715.

Ghez, C., Thach, W.T., 2000. The Cerebellum. In Principles of Neural Science, $4^{\text {th }}$ edition, Kandel, E.R., Schwartz, J.H., Jessell, T.M., ed. McGraw-Hill, U.S.A., pp. 833-852. 
Gilbert, A.K., Hosztafi, S., Mahurter, L., Pasternak, G.W., 2004. Pharmacological characterization of dihydromorphine, 6-acetyldihydromorphine and dihydroheroin analgesia and their differentiation from morphine. Eur. J. Pharmacol. 492, 123-130.

Glazewski, S., Fox, K., 1996. The time-course of experience-dependent synaptic potentiation and depression in barrel cortex of adolescent rats. J. Neurophysiol. 75, $1714-1729$.

Goldberg, I.E., Rossi, G.C., Letchworth, S.R., Mathis, J.P., Ryan-Moro, J., Leventhal, L., Su, W., Emmel, D., Bolan, E.A., Pasternak, G.W., 1998. Pharmacological characterization of endomorphin-1 and endomorphin-2 in mouse brain. J. Pharmacol. Exp. Ther. 286, 1007-1013.

Goldowitz, D., Hamre, K., 1998. The cells and molecules that make a cerebellum. Trends Neurosci. 21, 375-382.

Gomes, I., Jordan, B.A., Gupta, A., Trapaidze, N., Nagy, V., Devi, L.A., 2000. Heterodimerization of mu and delta opioid receptors: a role in opiate synergy. J. Neurosci. 20, RC110: 1-5.

Gomes, I., Gupta, A., Filipovska, J., Szeto, H.H., Pintar, J.E., Devi, L.A., 2004. A role for heterodimerization of $\mathrm{mu}$ and delta opiate receptors in enhancing morphine analgesia. Proc. Natl. Acad. Sci. U.S.A. 101, 5135-5139.

Gould, E., Cameron, H.A., Daniels, D.C., Woolley, C.S., McEwen, B.S., 1992. Adrenal hormones suppress cell division in the adult rat dentate gyrus. J. Neurosci. 12, 36423650 .

Goto, T., Mitsuhashi, T., Takahashi, T., 2004. Altered patterns of neuron production in the p27 knockout mouse. Dev. Neurosci. 26, 208-217.

Götz, M., Huttner, W.B., 2005. The cell biology of neurogenesis. Nat. Rev. Mol. Cell Biol. $6,777-788$.

Gray, G.E., Sanes, J.R., 1992. Lineage of radial glia in the chicken optic tectum. Development 114, 271-283.

Gundersen, H.J., Jensen, E.B., 1987. The efficiency of systematic sampling in stereology and its prediction. J. Microsc. 147, 229-263.

Gundersen, H.J., Jensen, E.B., Kiêu, K., Nielsen, J., 1999. The efficiency of systematic sampling in stereology-reconsidered. J. Microsc. 193, 199-211.

Gurwell, J.A., Duncan, M.J., Maderspach, K., Stiene-Martin, A., Elde, R.P., Hauser, K.F., 1996. Kappa-opioid receptor expression defines a phenotypically distinct subpopulation of astroglia: relationship to $\mathrm{Ca} 2+$ mobilization, development, and the antiproliferative effect of opioids. Brain Res. 737, 175-187.

Hahn, E.F., Pasternak, G.W., 1982. Naloxonazine, a potent, long-lasting inhibitor of opiate binding sites. Life Sci. 31, 1385-1388.

Hamada-Kanazawa, M., Ishikawa, K., Nomoto, K., Uozumi, T., Kawai, Y., Narahara, M., Miyake, M., 2004a. Sox6 overexpression causes cellular aggregation and the neuronal differentiation of P19 embryonic carcinoma cells in the absence of retinoic acid. FEBS Lett. 560, 192-198.

Hamada-Kanazawa, M., Ishikawa, K., Ogawa, D., Kanai, M., Kawai, Y., Narahara, M., Miyake, M., 2004b. Suppression of Sox6 in P19 cells leads to failure of neuronal differentiation by retinoic acid and induces retinoic acid-dependent apoptosis. FEBS Lett. 577, 60-66. 
Handal, M., Grung, M., Skurtveit, S., Ripel, A., Morland, J., 2002. Pharmacokinetic differences of morphine and morphine-glucuronides are related in locomotor activity. Pharmacol. Biochem. Behav. 73, 883-892.

Hans, S.L., Jeremy, R.J., 2001. Postneonatal mental and motor development of infants exposed in utero to opioid drugs. Infant Ment. Health J. 22, 300-315.

Harburg, G.C., Hall, F.S., Harrist, A.V., Sora, I., Uhl, G.R., Eisch, A.J., 2007. Knockout of the mu opioid receptor enhances the survival of adult-generated hippocampal granule cell neurons. Neuroscience 144, 77-87.

Hart, S., Fischer, O.M., Ullrich, A., 2004. Cannabinoids induce cancer cell proliferation via tumor necrosis factor alpha-converting enzyme (TACE/ADAM17)-mediated transactivation of the epidermal growth factor receptor. Cancer Res. 64, 1943-1950.

Hartfuss, E., Galli, R., Heins, N., Gotz, M., 2001. Characterization of CNS precursor subtypes and radial glia. Dev. Biol. 229, 15-30.

Haseman, J.K., Hogan, M.D., 1975. Selection of the experimental unit in teratology studies. Teratology 12, 165-171.

Hattox, A.M., Nelson, S.B., 2007. Layer V neurons in mouse cortex projecting to different targets have distinct physiological properties. J. Neurophysiol. 98, 3330-3340.

Haubensak, W., Attardo, A., Denk, W., Huttner, W.B., 2004. Neurons arise in the basal neuroepithelium of the early mammalian telencephalon: a major site of neurogenesis. Proc. Natl. Acad. Sci. U.S.A. 101, 3196-3201.

Hauser, K.F., McLaughlin, P.J., Zagon, I.S., 1987. Endogenous opioids regulate dendritic growth and spine formation in developing rat brain. Brain Res. 416, 157-161.

Hauser, K.F., McLaughlin, P.J., Zagon, I.S., 1989. Endogenous opioid systems and the regulation of dendritic growth and spine formation. J. Comp. Neurol. 281, 13-22.

Hauser, K.F., Osborne, J.G., Stiene-Martin, A., Melner, M.H., 1990. Cellular localization of proenkephalin mRNA and enkephalin peptide products in cultured astrocytes. Brain Res. 522, 347-353.

Hauser, K.F., Stiene-Martin, A., 1991. Characterization of opioid-dependent glial development in dissociated and organotypic cultures of mouse central nervous system: critical periods and target specificity. Brain Res. Dev. Brain Res. 62, 245255.

Hauser, K.F., 1992. Morphine regulates DNA synthesis in rat cerebellar neuroblasts in vitro. Brain Res. Dev. Brain Res. 70, 291-297.

Hauser, K.F., Stiene-Martin, A., Mattson, M.P., Elde, R.P., Ryan, S.E., Godleske, C.C., 1996. Mu-opioid receptor induced $\mathrm{Ca} 2+$ mobilization and astroglial development: morphine inhibits DNA synthesis and stimulates cellular hypertrophy through a $\mathrm{Ca}(2+)$-dependent mechanism. Brain Res. 720, 191-203.

Hauser, K.F, Mangoura, D., 1998. Diversity of the endogenous opioid system in development. Novel signal transduction translates multiple extracellular signals into neural cell growth and differentiation. Prospect. Dev. Neurobiol. 5, 437-449.

Hauser, K.F., Houdi, A.A., Turbek, C.S., Elde, R.P., Maxson, W., 2000. Opioids intrinsically inhibit the genesis of mouse cerebellar granule neuron precursors in vitro: differential impact of mu and delta receptor activation on proliferation and neurite elongation. Eur. J. Neurosci. 12, 1281-1293.

Hendzel, M.J., Wei, Y., Mancini, M.A., Van Hooser, A., Ranalli, T., Brinkley, B.R., Bazett-Jones, D.P., Allis, C.D., 1997. Mitosis-specific phosphorylation of histone 
$\mathrm{H} 3$ initiates primarily within pericentromeric heterochromatin during G2 and spreads in an ordered fashion coincident with mitotic chromosome condensation. Chromosoma 106, 348-360.

Herrmann, H., Aebi, U., 2000. Intermediate filaments and their associates: multi-talented structural elements specifying cytoarchitecture and cytodynamics. Curr. Opin. Cell Biol. 12, 79-90.

Heyman, J.S., Williams, C.L., Burks, T.F., Mosberg, H.I., Porreca, F., 1988. Dissociation of opioid antinociception and central gastrointestinal propulsion in the mouse: studies with naloxonazine. J. Pharmacol. Exp. Ther. 245, 238-243.

Hirai, T., Chida, K., 2003. Protein kinase zeta (PKC zeta): activation mechanisms and cellular functions. J. Biochem. 133, 1-7.

Hockfield, S., McKay, R.D.G., 1985. Identification of major cell classes in the developing mammalian nervous system. J. Neurosci. 5, 3310-3328.

Hodge, R.D., D'Ercole, A.J., O'Kusky, J.R., 2004. Insulin-like growth factor-I accelerates the cell cycle by decreasing G1 phase length and increases cell cycle reentry in the embryonic cerebral cortex. J. Neurosci. 24, 10201-10210.

Holmes, M.M., Galea, L.A., 2002. Defensive behavior and hippocampal cell proliferation: differential modulation by naltrexone during stress. Behav. Neurosci. 116, 160-168.

Holson, R.R., Pearce, B., 1992. Principles and pitfalls in the analysis of prenatal treatment effects in multiparous species. Neurotoxicol. Teratol. 14, 221-228.

Howard, B., Chen, Y., Zecevic, N., 2006. Cortical progenitor cells in the developing human telencephalon. Glia 53, 57-66.

Hughes, J., 1975. Isolation of an endogenous compound from the brain with pharmacological properties similar to morphine. Brain Res. 88, 295-308.

Hughes, J., Smith, T.W., Kosterlitz, H.W., Fothergill, L.A., Morgan, B.A., Morris, H.R., 1975a. Identification of two related pentapeptides from the brain with potent opiate agonist activity. Nature 258, 577-579.

Hughes, J., Smith, T., Morgan, B., Fothergill, L., 1975b. Purification and properties of enkephalin - the possible endogenous ligand for the morphine receptor. Life Sci. $16,1753-1758$.

Hull, E.M., Dominguez, J.M., 2007. Sexual behavior in male rodents. Horm. Behav. 52, 45-55.

Hutchings, D.E., Hunt, H.F., Towey, J.P., Rosen, T.S., Gorinson, H.S., 1976. Methadone during pregnancy in the rat: dose level effects on maternal and perinatal mortality and growth in the offspring. J. Pharmacol. Exp. Ther. 197, 171-179.

Hutchings, D.E., Zmitrovich, A., Brake, S.C., Malowany, D., Church, S., Nero, T.J., 1992. Prenatal administration of methadone using the osmotic minipump: effects on maternal and offspring toxicity, growth, and behavior in the rat. Neurotoxicol. Teratol. 14, 65-71.

Hwang, C.K., Wu, X., Wang, G., Kim, C.S., Loh, H.H., 2003. Mouse mu opioid receptor distal promoter transcriptional regulation by SOX proteins. J. Biol. Chem. 278, 3742-3750.

Ilyinsky, O.B., Kozlova, M.V., Kondrikova, E.S., Kalentchuk, V.U., Titov, M.I., Bespalova, Z.D., 1987. Effects of opioid peptides and naloxone on nervous tissue in culture. Neuroscience 22, 719-735. 
Janecka, A., Fichna, J., Janecki, T., 2004. Opioid receptors and their ligands. Curr. Top. Med. Chem. 4, 1-17.

Jiang, Q., Takemori, A.E., Sultana, M., Portoghese, P.S., Bowen, W.D., Mosberg, H.I., Porreca, F., 1991. Differential antagonism of opioid delta antinociception by [DAla2,Leu5,Cys6]enkephalin and naltrindole 5'-isothiocyanate: evidence for delta receptor subtypes. J. Pharmacol. Exp. Ther. 257, 1069-1075.

Jordan, B.A., Devi, L.A., 1999. G-protein-coupled receptor heterodimerization modulates receptor function. Nature 399, 697-700.

Kahn, L., Alonso, G., Normand, E., Manzoni, O.J., 2005. Repeated morphine treatment alters polysialylated neural cell adhesion molecule, glutamate decarboxylase-67 expression and cell proliferation in the adult rat hippocampus. Eur. J. Neurosci. 21, 493-500.

Kaltenbach, K.A., 1994. Effects of in-utero opiate exposure: new paradigms for old questions. Drug Alcohol Depend. 36, 83-87.

Kandel, E.R., 2000. The Neurobiology of Behavior. In Principles of Neural Science, $4^{\text {th }}$ edition, Kandel, E.R., Schwartz, J.H., Jessell, T.M., ed. McGraw-Hill, U.S.A., pp. 5-18.

Keith, D.E., Murray, S.R., Zaki, P.A., Chu, P.C., Lissin, D.V., Kang, L., Evans, C.J., von Zastrow, M., 1996. Morphine activates opioid receptors without causing their rapid internalization. J. Biol. Chem. 271, 19021-19024.

Kent, J.L., Pert, C.B., Herkenham, M., 1982. Ontogeny of opiate receptors in rat forebrain: visualization by in vitro autoradiography. Brain Res. Dev. Brain Res. 2, 487-504.

Khachaturian, H., Alessi, N.E., Munfakh, N., Watson, S.J., 1983. Ontogeny of opioid and related peptides in the rat cns and pituitary: an immunocytochemical study. Life Sci. 33, 61-64.

Kieffer, B.L., Befort, K., Gaveriaux-Ruff, C., Hirth, C.G., 1992. The delta-opioid receptor: isolation of a cDNA by expression cloning and pharmacological characterization. Proc. Natl. Acad. Sci. U.S.A. 89, 12048-12052.

Kieffer, B.L., Gaveriaux-Ruff, C., 2002. Exploring the opioid system by gene knockout. Prog. Neurobiol. 66, 285-306.

Kim, E., Clark, A.L., Kiss, A., Hahn, J.W., Wesselschmidt, R., Coscia, C.J., Belcheva, M.M., 2006. $\mu$ - and $\kappa$-opioids induce the differentiation of embryonic stem cells to neural progenitors. J. Biol. Chem. 281, 33749-33760.

Kivell, B.M., Day, D.J., McDonald, F.J., Miller, J.H., 2004. Developmental expression of mu and delta opioid receptors in the rat brainstem: evidence for a postnatal switch in mu isoform expression. Brain Res. Dev. Brain Res. 148, 185-196.

Knapp, P.E., Maderspach, K., Hauser, K.F., 1998. Endogenous opioid system in developing normal and jimpy oligodendrocytes: mu and kappa opioid receptors mediate differential mitogenic and growth responses. Glia 22, 189-201.

Ko, J.L., Chen, H.C., Loh, H.H., 2002. Differential promoter usage of mouse mu-opioid receptor gene development. Brain Res. Mol. Brain Res. 104, 184-193.

Koehl, M., Meerlo, P., Gonzales, D., Rontal, A., Turek, F.W., Abrous, D.N., 2008. Exercise-induced promotion of hippocampal cell proliferation requires betaendorphin. FASEB J. 22, 2253-2262. 
Kolodziej, A., Stumm, R., Becker, A., Höllt, V., 2008. Endogenous opioids inhibit ischemia-induced generation of immature hippocampal neurons via the mu-opioid receptor. Eur. J. Neurosci. 27, 1311-1319.

Kornblum, H.I., Loughlin, S.E., Leslie, F.M., 1987. Effects of morphine on DNA synthesis in neonatal rat brain. Dev. Brain Res. 31, 45-52.

Kornack, D.R., Rakic, P., 1998. Changes in cell-cycle kinetics during the development and evolution of primate neocortex. Proc. Natl. Acad. Sci. U.S.A. 95, 1242-1246.

Kriegstein, A.R., Noctor, S.C., 2004. Patterns of neuronal migration in the embryonic cortex. Trends Neurosci. 27, 392-399.

LaGasse, L.L., Messinger, D., Lester, B.M., Seifer, R., Tronick, E.Z., Bauer, C.R., Shankaran, S., Bada, H.S., Wright, L.L., Smeriglio, V.L., Finnegan, L.P., Maza, P.L., Liu, J., 2003. Prenatal drug exposure and maternal and infant feeding behaviour. Arch. Dis. Child. Fetal Neonatal Ed. 88, 391-399.

Lavdas, A.A., Grigoriou, M., Pachnis, V., Parnavelas, J.G., 1999. The medial ganglionic eminence gives rise to a population of early neurons in the developing cerebral cortex. J. Neurosci. 19, 7881-7888.

Law, P.Y., Erickson-Herbrandson, L.J., Zha, Q.Q., Solberg, J., Chu, J., Sarre, A., Loh, H.H., 2005. Heterodimerization of mu- and delta-opioid receptors occurs at the cell surface only and requires receptor-G protein interactions. J. Biol. Chem. 280, 11152-11164.

Lejeune, C., Simmat-Durand, L., Gourarier, L., Aubisson, S.; Groupe d'Etudes Grossesse et Addictions (GEGA)., 2006. Prospective multicenter observational study of 260 infants born to 259 opiate-dependent mothers on methadone or high-dose buprenophine substitution. Drug Alcohol Depend. 82, 250-257.

Leslie, F.M., Kosterlitz, H.W., 1979. Comparison of binding of [3H]-methionineenkephalin, $[3 \mathrm{H}]$-naltrexone and $[3 \mathrm{H}]$-dihydromorphine in the mouse vas deferens and the myenteric plexus and brain of the ginea pig. Eur. J. Pharmacol. 56, 379-383.

Leslie, F., Loughlin, S., 1993. Ontogeny and Plasticity of Opioid Systems. In The Neurobiology of Opiates, Hammer, R.P., ed. CRC Press, Boca Raton, FL, pp. 85123.

Leslie, F.M., Chen, Y., Winzer-Serhan, U.H., 1998. Opioid receptor and peptide mRNA expression in proliferative zones of fetal rat central nervous system. Can. J. Physiol. Pharmacol. 76, 284-293.

Lester, B.M., ElSohly, M., Wright, L.L., Smeriglio, V.L., Verter, J., Bauer, C.R., Shankaran, S., Bada, H.S., Walls, H.H., Huestis, M.A., Finnegan, L.P., Maza, P.L., 2001. The Maternal Lifestyle Study: drug use by meconium toxicology and maternal self-report. Pediatrics 107, 309-317.

Lester, B.M., Tronick, E.Z., LaGasse, L., Seifer, R., Bauer, C.R., Shankaran, S., Bada, H.S., Wright, L.L., Smeriglio, V.L., Lu, J., Finnegan, L.P., Maza, P.L., 2002. The maternal lifestyle study: effects of substance exposure during pregnancy on neurodevelopmental outcome in 1-month-old infants. Pediatrics 110, 1182-1192.

Lester, B.M., Lagasse, L., Seifer, R., Tronick, E.Z., Bauer, C.R., Shankaran, S., Bada, H.S., Wright, L.L., Smeriglio, V.L., Liu, J., Finnegan, L.P., Maza, P.L., 2003. The Maternal Lifestyle Study (MLS): effects of prenatal cocaine and/or opiate exposure on auditory brain response at one month. J. Pediatr. 142, 279-285. 
Letinic, K., Zoncu, R., Rakic, P., 2002. Origin of GABAergic neurons in the human neocortex. Nature 417, 645-649.

Levine, A.S., Billington, C.J., 2004. Opioids as agents of reward-related feeding: a consideration of the evidence. Physiol. Behav. 82, 57-61.

Levitt, P., Rakic, P., 1980. Immunoperoxidase localization of glial fibrillary acidic protein in radial glial cells and astrocytes of the developing rhesus monkey brain. J. Comp. Neurol. 193, 815-840.

Lewis, J.W., Husbands, S.M., 2004. The orvinols and related opioids - high affinity ligands with diverse efficacy profiles. Curr. Pharm. Des. 10, 717-732.

Li, S., Zhu, J., Chen, C., Chen, Y.W., Deriel, J.K., Ashby, B., Liu-Chen, L.Y., 1993. Molecular cloning and expression of a rat kappa opioid receptor. Biochem. J. 295, 629-633.

Li, Z., Theus, M.H., Wei, L., 2006. Role of ERK 1/2 signaling in neuronal differentiation of cultured embryonic stem cells. Dev. Growth Differ. 48, 513-523.

Li, Q., Lee, J.A., Black, D.L. 2007. Neuronal regulation of alternative pre-mRNA splicing. Nat. Rev. Neurosci. 8, 819-831.

Liang, Y., Mestek, A., Yu, L., Carr, L.G., 1995. Cloning and characterization of the promoter region of the mouse mu opioid receptor gene. Brain Res. 679, 82-88.

Lifschitz, M.H., Wilson, G.S., Smith, E.O., Desmond, M.M., 1985. Factors affecting head growth and intellectual function in children of drug addicts. Pediatrics 75, 269-274.

Ling, G.S., Pasternak, G.W., 1982. Morphine catalepsy in the rat: involvement of mu 1 (high affinity) opioid binding sites. Neurosci. Lett. 193-196.

Ling, G.S., Pasternak, G.W., 1983. Spinal and supraspinal opioid analgesia in the mouse: the role of subpopulations of opioid binding sites. Brain Res. 271, 152-156.

Ling, G.S., Spiegel, K., Nishimura, S.L., Pasternak, G.W., 1983. Dissociation of morphine's analgesic and respiratory depressant actions. Eur. J. Pharmacol. 86, 487488.

Ling, G.S., MacLeod, J.M., Lee, S., Lockhart, S.H., Pasternak, G.W., 1984. Separation of morphine analgesia from physical dependence. Science 226, 462-464.

Ling, G.S., Spiegel, K., Lockhart, S.H., Pasternak, G.W., 1985. Separation of opioid analgesia from respiratory depression: evidence for different receptor mechanisms. J. Pharmacol. Exp. Ther. 232, 149-155.

Liu-Chen, L.Y., Chen, C., Phillips, C.A., 1993. Beta-[3H]funaltrexamine-labeled muopioid receptors: species variations in molecular mass and glycosylation by complex-type, N-linked oligosaccharides. Mol. Pharmacol. 44, 749-756.

Lois, C., Alvarez-Buylla, A., 1993. Proliferating subventricular zone cells in the adult mammalian forebrain can differentiate into neurons and glia. Proc. Natl. Acad. Sci. U.S.A. 90, 2074-2077.

López-Sánchez, N., Müller, U., Frade, J.M., 2005. Lengthening of G2/mitosis in cortical precursors from mice lacking beta-amyloid precursor protein. Neuroscience 130, 51-60.

Lorber, B.A., Freitag, S.K., Bartolome, J.V., 1990. Effects of beta-endorphin on DNA synthesis in brain regions of preweanling rats. Brain Res. 531, 329-332.

Lord, J.A., Waterfield, A.A., Hughes, J., Kosterlitz, H.W., 1977. Endogenous opioid peptides: multiple agonists and receptors. Nature 267, 495-499. 
Luan, Y., Xu, W., 2007. The structure and main functions of aminopeptidase N. Curr. Med. Chem. 14, 639-647.

Lukaszewicz, A., Savatier, P., Cortay, V., Giroud, P., Huissoud, C., Berland, M., Kennedy, H., Dehay, C., 2005. G1 phase regulation, area-specific cell cycle control, and cytoarchitectonics in the primate cortex. Neuron 47, 353-364.

Malatesta, P., Hartfuss, E., Gotz, M., 2000. Isolation of radial glial cells by fluorescentactivated cell sorting reveals a neuronal lineage. Development 127, 5253-5263.

Malatesta, P., Hack, M.A., Hartfuss, E., Kettenmann, H., Klinkert, W., Kirchhoff, F., Gotz, M., 2003. Neuronal or glial progeny: regional differences in radial glia fate. Neuron 37, 751-764.

Mandyam C.D., Norris R.D., Eisch A.J., 2004. Chronic morphine induces premature mitosis of proliferating cells in the adult mouse subgranular zone. J. Neurosci. Res. 76, 783-794.

Mansour, A., Fox, C.A., Burke, S., Meng, F., Thompson, R.C., Akil, H., Watson, S.J., 1994. Mu, delta, and kappa opioid receptor mRNA expression in the rat CNS: an in situ hybridization study. J. Comp. Neurol. 350, 412-438.

Mansour, A., Fox, C.A., Burke, S., Akil, H., Watson, S.J., 1995. Immunohistochemical localization of the cloned mu opioid receptor in the rat CNS. J. Chem. Neuroanat. 8, 283-305.

Markham, J.K., Emmerson, J.L., Owen, N.V., 1971. Teratogenicity studies of methadone $\mathrm{HCl}$ in rats and rabbits. Nature 233, 342-343.

Martin, W.R., 1967. Opioid antagonists. Pharmacol. Rev. 19, 463-521.

Martin, W.R., Eades, C.G., Thompson, J.A., Huppler, R.E., Gilbert, P.E., 1976. The effects of morphine- and nalorphine- like drugs in the nondependent and morphinedependent chronic spinal dog. J. Pharmacol. Exp. Ther. 197, 517-532.

Matejusová, I., Felix, B., Sorsa-Leslie, T., Gilbey, J., Noble, L.R., Jones, C.S., Cunningham, C.O., 2006. Gene expression profiles of some immune relevant genes from skin of susceptible and responding Atlantic salmon (Salmo salar L.) infected with Gyrodactylus salaris (Monogenea) revealed by suppressive subtractive hybridisation. Int. J. Parasitol. 36, 1175-1183.

Matsugami, T.R., Tanemura, K., Mieda, M., Nakatomi, R., Yamada, K., Kondo, T., Ogawa, M., Obata, K., Watanabe, M., Hashikawa, T., Tanaka, K., 2006. Indispensability of the glutamate transporters GLAST and GLT1 to brain development. Proc. Natl. Acad. Sci. U.S.A. 103, 12161-12166.

Mattia, A., Vanderah, T., Mosberg, H.I., Porreca, F., 1991. Lack of antinociceptive crosstolerance between [D-Pen2, D-Pen5]enkephalin and [D-Ala2]deltorphin II in mice: evidence for delta receptor subtypes. J. Pharmacol. Exp. Ther. 258, 583-587.

McCarthy, M., Turnbull, D.H., Walsh, C.A., Fishell, G., 2001. Telencephalic neural progenitors appear to be restricted to regional and glial fates before the onset of neurogenesis. J. Neurosci. 21, 6772-6781.

McConnell, S.K., 1988. Fates of visual cortical neurons in the ferret after isochronic and heterochronic transplantation. J. Neurosci. 8, 945-974.

Mekel-Bobrov, N., Gilbert, S.L., Evans, P.D., Vallender, E.J., Anderson, J.R., Hudson, R.R., Tishkoff, S.A., Lahn, B.T., 2005. Ongoing adaptive evolution of ASPM, a brain size determinant in Homo sapiens. Science 309, 1720-1722. 
Melchiorri, P., Negri, L., 1996. The dermorphin peptide family. Gen. Pharmac. 21, 10991107.

Melner, M.H., Low, K.G., Allen, R.G., Nielsen, C.P., Young, S.L., Saneto, R.P., 1990. The regulation of proenkephalin expression in a distinct population of glial cells. EMBO J. 9, 791-796.

Meloche, S., Pouyssegur, J., 2007. The ERK1/2 mitogen-activated protein kinase pathway as a master regulator of the G1- to S-phase transition. Oncogene 26, 3227-3239.

Meng, F., Xie, G.X., Thompson, R.C., Mansour, A., Goldstein, A., Watson, S.J., Akil, H., 1993. Cloning and pharmacological characterization of a rat kappa opioid receptor. Proc. Natl. Acad. Sci. U.S.A. 90, 9954-9958.

Merkle, F.T., Tramontin, A.D., García-Verdugo, J.M., Alvarez-Buylla, A., 2004. Radial glia give rise to adult neural stem cells in the subventricular zone. Proc. Natl. Acad. Sci. U.S.A. 101, 17528-17532.

Messier, P.E., Auclair, C., 1974. Effect of cytochalasin B on interkinetic nuclear migration in the chick embryo. Dev. Biol. 36, 218-223.

Messing, R.B., Dodge, C., Waymire, J.C., Lynch, G.S., Deadwyler, S.A., 1979. Morphine induced increases in the incorporation of $3 \mathrm{H}$-thymidine into brain striatal DNA. Brain Res. Bull. 4, 615-619.

Messinger, D.S., Bauer, C.R., Das, A., Seifer, R., Lester, B.M., Lagasse, L.L., Wright, L.L., Shankaran, S., Bada, H.S., Smeriglio, V.L., Langer, J.C., Beeghly, M., Poole, W.K., 2004. The maternal lifestyle study: cognitive, motor, and behavioral outcomes of cocaine-exposed and opiate-exposed infants through three years of age. Pediatrics $113,1677-1685$.

Meunier, J.C., Zajac, J.M., 1979. Cerebellar opiate receptors in lagomorphs. Demonstration, characterization and regional distribution. Brain Res. 168, 311-321.

Meunier, J.C., 1997. Nociceptin/orphanin FQ and the opioid receptor-like ORL1 receptor. Eur. J. Pharmacol. 340, 1-15.

Mignat, C., Wille, U., Ziegler, A., 1995. Affinity profiles of morphine, codeine, dihydrocodeine and their glucuronides at opioid receptor subtypes. Life Sci. 56, 793-799.

Miller, C.R.D., O'Steen, W.K., Deadwyler, S.A., 1982. Effect of morphine on 3Hthymidine incorporation in the subependyma of the rat: an autoradiographic study. J. Comp. Neurol. 208, 209-214.

Min, B.H., Augustin, L.B., Felsheim, R.F., Fuchs, J.A., Loh, H.H., 1994. Genomic structure analysis of promoter sequence of a mouse mu opioid receptor gene. Proc. Natl. Acad. Sci. U.S.A. 91, 9081-9085.

Minami, M., Toya, T., Katao, Y., Maekawa, K., Nakamura, S., Onogi, T., Kaneko, S., Satoh, M., 1993. Cloning and expression of a cDNA for the rat kappa-opioid receptor. FEBS Lett. 329, 291-295.

Mitsuhashi, T., Aoki, Y., Eksioglu, Y.Z., Takahashi, T., Bhide, P.G., Reeves, S.A., Caviness, V.S. Jr., 2001. Overexpression of p27Kip1 lengthens the G1 phase in a mouse model that targets inducible gene expression to central nervous system progenitor cells. Proc. Natl. Acad. Sci. U.S.A. 98, 6435-6440.

Miyama, S., Takahashi, T., Nowakowski, R.S., Caviness, V.S., 1997. A gradient in the duration of the G1 phase in the murine neocortical proliferative epithelium. Cereb. Cortex 7, 678-689. 
Miyata, T., Kawaguchi, A., Okano, H., Ogawa, M., 2001. Asymmetric inheritance of radial glial fibres of cortical neurons. Neuron 31, 727-741.

Miyata, T., Kawaguchi, A., Saito, K., Kawano, M., Muto, T., Ogawa, M., 2004. Asymmetric production of surface-dividing and non-surface-dividing cortical progenitor cells. Development 131, 3133-3145.

Mizutani, K., Gaiano, N., 2006. Chalk one up for 'nature' during neocortical neurogenesis. Nat. Neurosci. 9, 717-718.

Mo, Z., Moore, A.R., Filipovic, R., Ogawa, Y., Kazuhiro, I., Antic, S.D., Zecevic, N., 2007. Human cortical neurons originate from radial glia and neuron-restricted progenitors. J. Neurosci. 27, 4132-4145.

Moles, A., Kieffer, B.L., D’Amato, F.R., 2004. Deficit in attachment behavior in mice lacking the mu-opioid receptor gene. Science 304, 1983-1986.

Mollick, J.A., Hodi, F.S., Soiffer, R.J., Nadler, L.M., Dranoff, G., 2003. MUC1-like tandem repeat proteins are broadly immunogenic in cancer patients. Cancer Immun. 17,3 .

Molnar, Z., Cheung, A.F.P, 2006. Towards the classification of subpopulations of layer V pyramidal projection neurons. Neurosci. Res. 55, 105-115.

Mori, T., Ito, S., Narita, M., Suzuki, T., Sawaguchi, T., 2004. Combined effects of psychostimulants and morphine on locomotor activity in mice. J. Pharmacol. Sci. 96, 450-458.

Mrkusich, E.M., Kivell, B.M., Miller, J.H., Day, D.J., 2004. Abundant expression of mu and delta opioid receptor mRNA and protein in the cerebellum of the fetal, neonatal, and adult rat. Brain Res. Dev. Brain Res. 148, 213-222.

Murciano, A., Zamora, J., Lopez-Sanchez, J., Frade, J.M., 2002. Interkinetic nuclear movement may provide spatial clues to the regulation of neurogenesis. Mol. Cell Neurosci. 21, 285-300.

Nadarajah, B., Alifragis, P., Wong, R.O., Parnavelas, J.G., 2003. Neuronal migration in the developing cerebral cortex: observations based on real-time imaging. Cereb. Cortex 13, 607-611.

Narita, M., Kuzumaki, N., Miyatake, M., Sato, F., Wachi, H., Seyama, Y., Suzuki, T., 2006. Role of delta-opioid receptor function in neurogenesis and neuroprotection. J. Neurochem. 97, 1494-1505.

Nestler, E.J., 2004. Historical review: molecular and cellular mechanisms of opiate and cocaine addiction. Trends Pharmacol. Sci. 25, 210-218.

Noctor, S.C., Flint, A.C., Weissman, T.A., Dammerman, R.S., Kriegstein, A.R., 2001. Neurons derived from radial glial cells establish radial units in neocortex. Nature 409, 714-720.

Noctor, S.C., Martínez-Cerdeño, V., Ivic, L., Kriegstein, A.R., 2004. Cortical neurons arise in symmetric and asymmetric division zones and migrate through specific phases. Nat. Neurosci. 7, 136-144.

Noctor, S.C., Martínez-Cerdeño, V., Kriegstein, A.R., 2007. Contribution of intermediate progenitor cells to cortical histogenesis. Arch. Neurol. 64, 639-642.

Nudo, R.J., 2006. Mechanisms for recovery of motor function following cortical damage. Curr. Opin. Neurobiol. 16, 638-644.

Oczko-Wojciechowska, M., Włoch, J., Wiench, M., Fujarewicz, K., Simek, K., Gala, G., Gubała, E., Szpak-Ulczok, S., Jarzab, B., 2006. [Gene expression profile of 
medullary thyroid carcinoma--preliminary results] Article in Polish. Endokrynol. Pol. 57,420-426.

Olianas, M.C., Concas, D., Onali, P., 2006. Agonist activity of naloxone benzoylhydrazone at recombinant and native opioid receptors. Br. J. Pharmacol. 147, 360-370.

Opanashuk, L.A., Hauser, K.F., 1998. Opposing actions of the EGF family and opioids: heparin binding-epidermal growth factor (HB-EGF) protects mouse cerebellar neuroblasts against the antiproliferative effect of morphine. Brain Res. 804, 87-94.

Ornoy, A., Michailevskaya, V., Lukashov, I., Bar-Hamburger, R., Harel, S., 1996. The developmental outcome of children born to heroin-dependent mothers, raised at home or adopted. Child Abuse Negl. 20, 385-396.

Osborne, J.G., Kindy, M.S., Hauser, K.F., 1991. Expression of proenkephalin mRNA in developing cerebellar cortex of the rat: expression levels coincide with maturational gradients in Purkinje cells. Brain Res. Dev. Brain Res. 63, 63-69.

Osborne, J.G., Kindy, M.S., Spruce, B.A., Hauser, K.F., 1993. Ontogeny of proenkephalin mRNA and enkephalin peptide expression in the cerebellar cortex of the rat: spatial and temporal patterns of expression follow maturational gradients in the external granular layer and in Purkinje cells. Brain Res. Dev. Brain Res. 76, 1-12.

Palmer, A.K., 1974. Statistical analysis and choice of sampling units. Teratology 10, 301302.

Pan, Y.X., Xu, J., Mahurter, L., Bolan, E., Xu, M., Pasternak, G.W., 2001. Generation of the mu opioid receptor (MOR-1) protein by three new splice variants of the Oprm gene. Proc. Nat. Acad. Sci. U.S.A. 98, 14084-14089.

Pan, Y.X., 2002. Identification and characterization of a novel promoter of the mouse opioid receptor gene (Oprm) that generates eight splice variants. Gene 295, 97-108.

Pan, Y.X., Xu, J., Mahurter, L., Xu, M., Gilbert, A.K., Pasternak, G.W., 2003. Identification and characterization of two new human mu opioid receptor splice variants, hMOR-1O and hMOR-1X. Biochem. Biophys. Res. Commun. 301, 10571061.

Pan, Y.X., 2005. Diversity and complexity of the mu opioid receptor gene: alternative premRNA splicing and promoters. DNA Cell Biol. 24, 736-750.

Pan, L., Xu, J., Yu, R., Xu, M.M., Pan, Y.X., Pasternak, G.W., 2005a. Identification and characterization of six new alternatively spliced variants of the human mu opioid receptor gene, Oprm. Neuroscience 133, 209-220.

Panagiotou, S., Bakogeorgou, E., Papakonstanti, E., Hatzoglou, A., Wallet, F., Dussert, C., Stournaras, C., Martin, P.M., Castanas, E., 1999. Opioid agonists modify breast cancer cell proliferation by blocking cells to the G2/M phase of the cycle: involvement of cytoskeletal elements. J. Cell. Biochem. 73, 204-211.

Parnavelas, J.G., 2000. The origin and migration of cortical neurones: new vistas. Trends Neurosci. 23, 126-131.

Pasternak, D.A., Pan, L., Xu, J., Yu, R., Xu, M-M., Pasternak, G.W., Pan, Y-X., 2004. Identification of three new alternatively spliced variants of the rat mu opioid receptor gene: dissociation of affinity and efficacy. J. Neurochem. 91, 881-890.

Pasternak, G.W., Snyder, S.H., 1975. Identification of novel high affinity opiate receptor binding in rat brain. Nature 253, 563-565.

Pasternak, G.W., 2001. Insights into mu opioid pharmacology the role of mu opioid receptor subtypes. Life Sci. 68, 2213-2219. 
Pasternak, G.W., 2004. Multiple opiate receptors: de’ja`vu all over again. Neuropharmacology 47, 312-323.

Paul, D., Bodnar, R.J., Gistrak, M.A., Pasternak, G.W., 1989. Different mu receptor subtypes mediate spinal and supraspinal analgesia in mice. Eur. J. Pharmacol. 168, 307-314.

Persson, A.I., Thorlin, T., Bull, C., Eriksson, P.S., 2003a. Opioid-induced proliferation through the MAPK pathway in cultures of adult hippocampal progenitors. Mol. Cell. Neurosci. 23, 360-372.

Persson, A.I., Thorlin, T., Bull, C., Zarnegar, P., Ekman, R., Terenius, L., Eriksson, P.S., 2003b. Mu- and delta-opioid receptor antagonists decrease proliferation and increase neurogenesis in cultures of rat adult hippocampal progenitors. Eur. J. Neurosci. 17, 1159-1172.

Persson, A.I., Naylor, A.S., Jonsdottir, I.H., Nyberg, F., Eriksson, P.S., Thorlin, T., 2004. Differential regulation of hippocampal progenitor proliferation by opioid receptor antagonists in running and non-running spontaneously hypertensive rats. Eur. J. Neurosci. 19, 1847-1855.

Peters, M.A., 1977. The effect of maternally administered methadone on brain development in the offspring. J. Pharmacol. Exp. Ther. 203, 340-346.

Peters, I.R., Helps, C.R., Hall, E.J., Day, M.J., 2004. Real-time RT-PCR: considerations for efficient and sensitive assay design. J. Immunol. Methods 286, 203-217.

Petrie, B.F., 1993. Naltrexone does not increase brain and body development in rats. Neurotoxicol. Teratol. 15, 275-277.

Pinto, L., Götz, M., 2007. Radial glial cell heterogeneity-the source of diverse progeny in the CNS. Prog. Neurobiol. 83, 2-23.

Porteus, M.H., Bulfone, A., Liu, J.K., Puelles, L., Lo, L.C., Rubenstein, J.L., 1994. DLX-2, MASH-1, and MAP-2 expression and bromodeoxyuridine incorporation define molecularly distinct cell populations in the embryonic mouse forebrain. J. Neurosci. 14, 6370-6383.

Prenzel, N., Zwick, E., Daub, H., Leserer, M., Abraham, R., Wallasch, C., Ullrich, A., 1999. EGF receptor transactivation by G-protein-coupled receptors requires metalloproteinase cleavage of proHB-EGF. Nature 402, 884-888.

Ra, S.M., Kim, H., Jang, M.H., Shin, M.C., Lee, T.H., Lim, B.V., Kim, C.J., Kim, E.H., Kim, K.M., Kim, S.S., 2002. Treadmill running and swimming increase cell proliferation in the hippocampal dentate gyrus of rats. Neurosci. Lett. 333, 123-126.

Raff, M.C., Barres, B.A., Burne, J.F., Coles, H.S., Ishizaki, Y., Jacobson, M.D., 1993. Programmed cell death and the control of cell survival: lessons from the nervous system. Science 262, 695-700.

Rakic, P., 1972. Mode of cell migration to the superficial layers of fetal monkey neocortex. J. Comp. Neurol. 145, 61-84.

Rakic, P., 2006. A century of progress in corticoneurogenesis: from silver impregnation to genetic engineering. Cereb. Cortex 16, Suppl 1:i3-17

Raman, M., Chen, W., Cobb, M.H., 2007. Differential regulation and properties of MAPKs. Oncogene 26, 3100-3112.

Raynor, K., Kong, H., Chen, Y., Yasuda, K., Yu, L. Bell, G.I., Reisine, T., 1994. Pharmacological characterization of the cloned kappa-, delta-, and mu-opioid receptors. Mol. Pharmacol. 45, 330-334. 
Reznikov, K., Hauser, K.F., Nazarevskaja, G., Trunova, Y., Derjabin, V., Bakalkin, G., 1999. Opioids modulate cell division in the germinal zone of the late embryonic neocortex. Eur. J. Neurosci. 11, 2711-2719.

Rios, C., Gomes, I., Devi, L.A., 2006. Mu opioid and CB1 cannabinoid receptor interactions: reciprocal inhibition of receptor signaling and neuritogenesis. Br. J. Pharmacol. 148, 387-395.

Rius, R.A., Barg, J., Bem, W.T., Coscia, C.J., Loh, Y.P., 1991. The prenatal development profile of expression of opioid peptides and receptors in the mouse brain. Brain Res. Dev. Brain Res. 58, 237-241.

Robertson, S.A., Andrew, S.E., 2003. Presence of opioid growth factor and its receptor in the normal dog, cat and horse cornea. Vet. Ophthalmol. 6, 131-134.

Romanko, M.J., Rola, R., Fike, J.R., Szele, F.G., Dizon, M.L., Felling, R.J., Brazel, C.Y., Levison, S.W., 2004. Roles of the mammalian subventricular zone in cell replacement after brain injury. Prog. Neurobiol. 74, 77-99.

Roovers, K., Assoian, R.K., 2000. Integrating the MAP kinase signal into the G1 phase cell machinery. BioEssays 22, 818-826.

Rosen, T.S., Johnson, H.L., 1982. Children of methadone-maintained mothers: follow-up to 18 months of age. J. Pediatr. 101, 192-196.

Rothman, R.B., Bykov, V., de Costa, B.R., Jacobson, A.E., Rice, K.C., Brady, L.S., 1990. Interaction of endogenous opioid peptides and other drugs with four kappa opioid binding sites in guinea pig brain. Peptides 11, 311-331.

Rozenfeld, R., Devi, L.A., 2007. Receptor heterodimerization leads to a switch in signaling: beta-arrestin2-mediated ERK activation by mu-delta opioid receptor heterodimers. FASEB J. 21, 2455-2465.

Rozengurt, E., 2007. Mitogenic signaling pathways induced by G protein-coupled receptors. J. Cell. Physiol. 213, 589-602.

Ruzicka, B.B., Fox, C.A., Thompson, R.C., Meng, F., Watson, S.J., Akil, H., 1995. Primary astroglial cultures derived from several rat brain regions differentially express mu, delta and kappa opioid receptor mRNA. Brain Res. Mol. Brain Res. 34, 209-220.

Sanai, N., Berger, M.S., Garcia-Verdugo, J.M., Alvarez-Buylla, A., 2007. Comment on "Human neuroblasts migrate to the olfactory bulb via a lateral ventricular extension". Science 318, 393.

Sandberg, M., Kallstrom, M., Muhr, J., 2005. Sox 21 promotes the progression of vertebrate neurogenesis. Nat. Neurosci. 8, 995-1001.

Sargeant, T.J., Day, D.J., Mrkusich, E.M., Foo, D.F., Miller, J.H., 2007. Mu opioid receptors are expressed on radial glia but not migrating neuroblasts in the late embryonic mouse brain. Brain Res. 1175, 28-39.

Sargeant, T.J., Day, D.J., Miller, J.H., Steel, R.W.J., 2008. Acute in-utero morphine exposure slows $\mathrm{G} 2 / \mathrm{M}$ phase transition in radial glial and basal progenitor cells in the dorsal telencephalon of the E15.5 embryonic mouse. Eur. J. Neurosci. In press.

Sauer, F.C., 1935. Mitosis in the neural tube. J. Comp. Neurol. 62, 377-405.

Sbarbati, A., Pizzini, F., Fabene, P.F., Nicolato, E., Marzola, P., Calderan, L., Simonati, A., Longo, L., Osculati, A., Beltramello, A., 2004. Cerebral cortex three-dimensional profiling in human fetuses by magnetic resonance imaging. J. Anat. 204, 465-474. 
Schmahl, W., Funk, R., Miaskowski, U., Plendl, J., 1989. Long-lasting effects of naltrexone, an opioid receptor antagonist, on cell proliferation in developing rat forebrain. Brain Res. 486, 297-300.

Schmechel, D.E., Rakic, P., 1979. A Golgi study of radial glial cells in developing monkey telencephalon: morphogenesis and transformation into astrocytes. Anat. Embryol. (Berl.) 156, 115-152.

Scholzen, T., Gerdes, J., 2000. The Ki-67 protein: from the known and the unknown. J. Cell. Physiol. 182, 311-322.

Schuller, A.G., King, M.A., Bolan, E., Pan, Y.X., Morgan, D.J., Chang, A., Czick, M.E., Unterwald, E.M., Pasternak, G.W., Pintar, J.E., 1999. Retention of heroin and morphine- 6 beta-glucuronide analgesia in a new line of mice lacking exon 1 of MOR-1. Nat. Neurosci. 2, 151-156.

Seatriz, J.V., Hammer, R.P., 1993. Effects of opiates on neuronal development in the rat cerebral cortex. Brain Res. Bull. 30, 523-527.

Shen, Q., Wang, Y., Dimos, J.T., Fasano, C.A., Phoenix, T.N., Lemischka, I.R., Ivanova, N.B., Stifani, S., Morrisey, E.E., Temple, S., 2006. The timing of cortical neurogenesis is encoded within lineages of individual progenitor cells. Nat. Neurosci. 9, 743-751.

Sheng, W.S., Hu, S., Herr, G., Ni, H.T., Rock, R.B., Gekker, G., Lokensgard, J.R., Peterson, P.K., 2007. Human neural precursor cells express functional kappa opioid receptors. J. Pharmacol. Exp. Ther. 322, 957-963.

Shibata, T., Yamada, K., Watanabe, M., Ikenaka, K., Wada, K., Tanaka, K., Inoue, Y., 1997. Glutamate transporter GLAST is expressed in the radial glia-astrocyte lineage of developing mouse spinal cord. J. Neurosci. 17, 9212-9219.

Shinoda, H., Marini, A.M., Cosi, C., Schwartz, J.P., 1989. Brain region and gene specificity of neuropeptide gene expression in cultured astrocytes. Science 245, 415-417.

Shinoda, H., Marini, A.M., Schwartz, J.P., 1992. Developmental expression of the proenkephalin and prosomatostatin genes in cultured cortical and cerebellar astrocytes. Brain Res. Dev. Brain Res. 67, 205-210.

Simantov, R., Snyder, S.H., 1976. Morphine-like peptides in mammalian brain: isolation, structure elucidation, and interactions with the opiate receptor. Proc. Natl. Acad. Sci. U.S.A. 73, 2515-2519.

Smart, I.H.M., 1973. Proliferative characteristics of the ependymal layer during the early development of the mouse neocortex: a pilot study based on recording the number, location and plane of cleavage of mitotic figures. J. Anat. 116, 67-91.

Smart, I.H., Dehay, C., Giroud, P., Berland, M., Kennedy, H., 2002. Unique morphological features of the proliferative zones and postmitotic compartments of the neural epithelium giving rise to striate and extrastriate cortex in the monkey. Cereb. Cortex 12, 37-53.

Snyder, S.H., Pasternak, G.W., 2003. Historical review: opioid receptors. Trends Pharmacol. Sci. 24, 198-205.

Soriano, E., del Rio, J.A., 2005. The cells of Cajal-Retzius: still a mystery one century after. Neuron 46, 389-394.

Spencer-Dene, B., Thorogood, P., Nair, S., Kenny, A.J., Harris, M., Henderson, B., 1994. Distribution of, and a putative role for, the cell-surface neutral metallo- 
endopeptidases during mammalian craniofacial development. Development 120, 3213-3226.

Spiegel, K., Kourides, I., Pasternak, G.W., 1982. Prolactin and growth hormone release by morphine in the rat: different receptor mechanisms. Science 217, 745-7.

Spruce, B.A., Curtis, R., Wilkin, G.P., Glover, D.M., 1990. A neuropeptide precursor in cerebellum: proenkephalin exists in subpopulations of both neurons and astrocytes. EMBO J. 9, 1787-1795.

Stiene-Martin, A., Hauser, K.F., 1990. Opioid-dependent growth of glial cultures: suppression of astrocyte DNA synthesis by met-enkephalin. Life Sci. 46, 91-98.

Stiene-Martin, A., Hauser, K.F., 1991. Glial growth is regulated by agonists selective for multiple opioid receptor types in vitro. J. Neurosci. Res. 29, 538-548.

Stiene-Martin, A., Osborne, J.G., Hauser, K.F., 1991a. Co-localization of proenkephalin mRNA using cRNA probes and a cell-type-specific immunocytochemical marker for intact astrocytes in vitro. J. Neurosci. Methods 36, 119-126.

Stiene-Martin, A., Gurwell, J.A., Hauser, K.F., 1991b. Morphine alters astrocyte growth in primary cultures of mouse glial cells: evidence for a direct effect of opiates on neural maturation. Brain Res. Dev. Brain Res. 60, 1-7.

Stiene-Martin, A., Hauser, K.F., 1993. Morphine suppresses DNA synthesis in cultured murine astrocytes from cortex, hippocampus and striatum. Neurosci. Lett. 157, 1-3.

Stiene-Martin, A., Mattson, M.P., Hauser, K.F., 1993. Opiates selectively increase intracellular calcium in developing type-1 astrocytes: role of calcium in morphineinduced morphologic differentiation. Brain Res. Dev. Brain Res. 76, 189-196.

Stiene-Martin, A., Zhou, R., Hauser, K.F., 1998. Regional, developmental and cell cycledependent differences in $\mu, \delta$, and $\kappa$-opioid receptor expression among cultured mouse astrocytes. Glia 22, 249-259.

Stiene-Martin, A., Knapp, P.E., Martin, K., Gurwell, J.A., Ryan, S., Thornton, S.R., Smith, F.L., Hauser, K.F., 2001. Opioid system diversity in developing neurons, astroglia and oligodendroglia in the subventricular zone and striatum: impact on gliogenesis in vivo. Glia 36, 78-88.

Subramanian, G., Paterlini, M.G., Portoghese, P.S., Ferguson, D.M., 2000. Molecular docking reveals a novel binding site model for fentanyl at the mu-opioid receptor. J. Med. Chem. 43, 381-391.

Takahashi, T., Nowakowski, R.S., Caviness, V.S. Jr., 1993. Cell cycle parameters and patterns of nuclear movement in the neocortical proliferative zone of the fetal mouse. J. Neurosci. 13, 820-833.

Takahashi, T., Nowakowski, R.S., Caviness, V.S. Jr., 1995a. The cell cycle of the pseudostratified ventricular epithelium of the embryonic murine cerebral wall. J. Neurosci. 15, 6046-6057.

Takahashi, T., Nowakowski, R.S., Caviness V.S. Jr, 1995b. Early ontogeny of the secondary proliferative population of the embryonic murine cerebral wall. J. Neurosci. 15, 6058-6068.

Takahashi, T., Nowakowski, R.S., Caviness, V.S. Jr, 1996a. The leaving or Q fraction of the murine cerebral proliferative epithelium: a general model of neocortical neurogenesis. J. Neurosci. 16, 6183-6196. 
Takahashi, T., Nowakowski, R.S., Caviness, V.S. Jr., 1996b. Interkinetic and migratory behavior of a cohort of neocortical neurons arising in the early embryonic murine cerebral wall. J. Neurosci. 16, 5762-5776.

Takahashi, T., Bhide, P.G., Goto, T., Miyama, S., Caviness, V.S. Jr, 1999. Proliferative behavior of the murine cerebral wall in tissue culture: cell cycle kinetics and checkpoints. Exp. Neurol. 156, 407-417.

Tamai, H., Shinohara, H., Takaki, M., Saito, K., Nishizawa, Y., Nomura, T., Osumi, N., 2007. Pax6 transcription factor is required for the interkinetic nuclear movement of neuroepithelial cells. Genes to Cells 12, 983-996.

Tao, P.L., Yeh, G.C., Su, C.H., Wu, Y.H., 2001. Co-administration of dextromethorphan during pregnancy and throughout lactation significantly decreases the adverse effects associated with chronic morphine administration in rat offspring. Life Sci. 69, 2439-2450.

Tarabykin, V., Stoykova, A., Usman, N., Gruss, P., 2001. Cortical upper layer neurons derive from the subventricular zone as indicated by Svet 1 gene expression. Development 128, 1983-1993.

Tarui, T., Takahashi, T., Nowakowski, R.S., Hayes, N.L., Bhide, P.G., Caviness, V.S., 2005. Overexpression of p27 Kip 1, probability of cell cycle exit, and laminar destination of neocortical neurons. Cereb. Cortex 15, 1343-1355.

Terenius, L., Wahlstrom, A., 1975. Search for an endogenous ligand for the opiate receptor. Acta. Physiol. Scand. 94, 74-81.

Thompson, R.C., Mansour, A., Akil, H., Watson, S.J., 1993. Cloning and pharmacological characterization of a rat mu opioid receptor. Neuron 11, 903-913.

Tian, M., Broxmeyer, H.E., Fan, Y., Lai, Z., Zhang, S., Aronica, S., Cooper, S., Bigsby, R.M., Steinmetz, R., Engle, S.J., Mestek, A., Pollock, J.D., Lehman, M.N., Jansen, H.T., Ying, M., Stambrook, P.J., Tischfield, J.A., Yu, L., 1997. Altered hematopoiesis, behavior, and sexual function in mu opioid receptor-deficient mice. J. Exp. Med. 185, 1517-1522.

Tocris Bioscience, 2007. Opioids. In Life Science Catalogue 2007. Tocris House, Bristol, UK, pp. 55-56.

Tong, Y., Chabot, J.G., Shen, S.H., O'Dowd, B.F., George, S.R., Quirion, R., 2000. Ontogenic profile of the expression of the mu opioid receptor gene in the rat telencephalon and diencephalon: an in situ hybridization study. J. Chem. Neuroanat. $18,209-222$.

Tramontin, A.D., García-Verdugo, J.M., Lim, D.A., Alvarez-Buylla, A., 2003. Postnatal development of radial glia and the ventricular zone (VZ): a continuum of the neural stem cell compartment. Cereb. Cortex 13, 580-587.

Tsai, J.W., Chen, Y., Kriegstein, A.R., Vallee, R.B., 2005. LIS1 RNA interference blocks neural stem cell division, morphogenesis, and motility at multiple stages. J. Cell Biol. 170, 935-945.

Ueno, M., Katayama, K., Yamauchi, H., Nakayama, H., Doi, K., 2006. Cell cycle progression is required for nuclear migration of neural progenitor cells. Brain Res., 1088, 57-67.

Urosevic, M., Oberholzer, P.A., Maier, T., Hafner, J., Laine, E., Slade, H., Benninghoff, B., Burg, G., Dummer, R., 2004. Imiquimod treatment induces expression of opioid 
growth factor receptor: a novel tumor antigen induced by interferon-alpha? Clin. Cancer Res. 10, 4959-4970.

Verney, C., Takahashi, T., Bhide, P.G., Nowakowski, R.S., Caviness, V.S. Jr., 2000. Independent controls for neocortical neuron production and histogenetic cell death. Dev. Neurosci. 22, 125-138.

Vértes, Z., Melegh, G., Vértes, M., Kovács, S., 1982. Effect of naloxone and D-met2-pro5enkephalinamide treatment on the DNA synthesis in the developing rat brain. Life Sci. 31, 119-126.

Vilijn, M.H., Vaysse, P.J., Zukin, R.S., Kessler, J.A., 1988. Expression of preproenkephalin mRNA by cultured astrocytes and neurons. Proc. Natl. Acad. Sci. U.S.A. 85, 65516555.

Vonkeman, H.E., Voorn, P., Brady, L.S., Berendse, H.W., Richfield, E.D., 1996. Opioid receptor ligand binding in the human striatum: II. Heterogeneous distribution of kappa opioid receptor labeled with [3H]Bremazocine. J. Comp. Neurol. 374, 223229.

Voorn, P., Brady, L.S., Berendse, H.W., Richfield, E.K., 1996. Densitometrical analysis of opioid receptor ligand binding in the human striatum-I. Distribution of $\mu$ opioid receptor defines shell and core of the ventral striatum. Neuroscience 75, 777-792.

Wainwright, P., 1998. Issues in design and analysis relating to the use of multiparous species in developmental nutritional studies. J. Nutr. 128, 661-663.

Wang, J.B., Imai, Y., Eppler, C.M., Gregor, P., Spivak, C.E., Uhl, G.R., 1993. Mu opiate receptor: cDNA cloning and expression. Proc. Natl. Acad. Sci. U.S.A. 90, 1023010234.

Wang, H.Q., Kampine, J.P., Tseng, L.F., 1996. Antisense oligodeoxynucleotide to a deltaopioid receptor messenger RNA selectively blocks the antinociception induced by intracerebroventricularly administered delta- but not mu-, epsilon- or kappa-opioid receptor agonists in the mouse. Neuroscience 75, 445-452.

Wang, D., Sadee, W., Quillan, J.M., 1999. Calmodulin binding to G protein-coupling domain of opioid receptors. J. Biol. Chem. 274, 22081-22088.

Wang, D., Sun, X., Bohn, L.M., Sadee, W., 2005. Opioid receptor homo- and heterodimerization in living cells by quantitative bioluminescence resonance energy transfer. Mol. Pharmacol. 67, 2173-2184.

Wang, X., Dow-Edwards, D., Anderson, V., Minkoff, H., Hurd, Y.L., 2006. Discrete opioid gene expression impairment in the human fetal brain associated with maternal marijuana use. Pharmacogenomics J. 6, 255-264.

Warwick, R., Williams, P.L., 1973. Embryology. In Gray's Anatomy, $35^{\text {th }}$ edition, Longman Group Ltd., Edinburgh, UK, pp. 132-142.

Wegner, M., Stolt, C.C., 2005. From stem cells to neurons and glia: a Soxist's view of neural development. Trends Neurosci. 28, 583-588.

Wei, L.N., Loh, H.H., 2002. Regulation of opioid receptor expression. Curr. Opin. Pharmacol. 2, 69-75.

Wei, G., Moss, J., Yuan, C.S., 2003. Opioid-induced immunosuppression: is it centrally mediated or peripherally mediated? Biochem. Pharmacol. 65, 1761-1766.

Weil, C.S., 1974. Choice of the number of sampling units in teratology. Teratology 10, 301.

Wetzker, R., Bohmer, F.D., 2003. Transactivation joins multiple tracks to the ERK/MAPK cascade. Nat. Rev. Mol. Cell Biol. 4, 651-657. 
Willson, N.J., Schneider, J.F., Roizin, L., Fleiss, J.F., Rivers, W., Demartini, J.E., 1976. Effects of methadone hydrochloride on the growth of organotypic cerebellar cultures prepared from methadone-tolerant and control rats. J. Pharmacol. Exp. Ther. 199, 368-374.

Wilson, G.S., Desmond, M.M., Wait, R.B., 1981. Follow-up of methadone-treated and untreated narcotic-dependent women and their infants: health, developmental, and social implications. J. Pediatr. 98, 716-722.

Winzer-Serhan, U.H., Chen, Y., Leslie, F.M., 2003. Expression of opioid peptides and receptors in striatum and substantia nigra during rat brain development. J. Chem. Neuroanat. 26, 17-36.

Wollemann, M., Benyhe, S., 2004. Non-opioid actions of opioid peptides. Life Sci. 75, 257-270.

Wolozin, B.L., Pasternak, G.W., 1981. Classification of multiple morphine and enkephalin binding sites in the central nervous system. Proc. Natl. Acad. Sci. U.S.A. 78, 61816185.

Xu, H., Ni, Q., Jacobson, A.E., Rice, K.C., Rothman, R.B., 1991. Preliminary ligand binding data for subtypes of the delta opioid receptor in rat brain membranes. Life Sci. 49, PL141-146.

Xu, B., Kim, S-T., Lim, D-S., Kastan, M. B., 2002. Two molecularly distinct G2/M checkpoints are induced by ionizing irradiation. Mol. Cell Biol., 22, 1049-1059.

Xu, J., Xu, M., Pan, Y.X., 2006. Characterizing exons 11 and 1 promoters of the mu opioid receptor (Oprm) gene in transgenic mice. BMC Mol. Biol. 7, 41-56.

Yin, D., Mufson, R.A., Wang, R., Shi, Y., 1999. Fas-mediated cell death promoted by opioids. Nature 397, 218.

Young, K.M., Fogarty, M., Kessaris, N., Richardson, W.D., 2007. Subventricular zone stem cells are heterogeneous with respect to their embryonic origins and neurogenic fates in the adult olfactory bulb. J. Neurosci. 27, 8286-8296.

Zagon, I.S., McLaughlin, P.J., 1977a. The effects of different schedules of methadone treatment on rat brain development. Exp. Neurol. 56, 538-552.

Zagon, I.S., McLaughlin, P.J., 1977b. Methadone and brain development. Experientia 33, 1486-1487.

Zagon, I.S., McLaughlin, P.J., 1983. Increased brain size and cellular content in infant rats treated with an opiate antagonist. Science 221, 1179-1180.

Zagon, I.S., Rhodes, R.E., McLaughlin, P.J., 1985. Distribution of enkephalin immunoreactivity in germinative cells of developing rat cerebellum. Science 227, 1049-1051.

Zagon, I.S., McLaughlin, P.J., 1986a. Opioid antagonist (naltrexone) modulation of cerebellar development: histological and morphometric studies. J. Neurosci. 6, 1424-1432.

Zagon, I.S., McLaughlin, P.J., 1986b. Opioid antagonist-induced modulation of cerebral and hippocampal development: histological and morphometric studies. Brain Res. 28, 233-246.

Zagon, I.S., McLaughlin, P.J., 1987. Endogenous opioid systems regulate cell proliferation in the developing rat brain. Brain Res. 412, 68-72.

Zagon, I.S., Isayama, T., McLaughlin, P.J., 1994. Preproenkephalin mRNA expression in the developing and adult rat brain. Brain Res. Mol. Brain Res. 21, 85-98. 
Zagon, I.S., Verderame, M.F., Allen, S.S., McLaughlin, P.J., 1999a. Cloning, sequencing, expression and function of a cDNA encoding a receptor for the opioid growth factor, [Met(5)]enkephalin. Brain Res. 849, 147-154.

Zagon, I.S., Wu, Y., McLaughlin, P.J., 1999b. Opioid growth factor and organ development in rat and human embryos. Brain Res. 839, 313-322.

Zagon, I.S., Verderame, M.F., McLaughlin, P.J., 2002. The biology of the opioid growth factor receptor (OGFr). Brain Res. Brain Res. Rev. 38, 351-376.

Zecevic, N., 2004. Specific characteristic of radial glia in the human fetal telencephalon. Glia 48, 27-35.

Zhang, Y., Pana, Y.X., Kolesnikovb, Y., Pasternak, G.W., 2006. Immunohistochemical labelling of the mu opioid receptor carboxy terminal splice variant mMOR-1B4 in the mouse central nervous system. Brain Res. 1099, 33-43.

Zhu, Y., Hsu, M.S., Pintar, J.E., 1998. Developmental expression of the mu, kappa and delta opioid receptor mRNAs in mouse. J. Neurosci. 18, 2538-2549.

Zimprich, A., Simon, T., Hollt, V., 1995. Cloning and expression of an isoform of the rat mu opioid receptor (rMOR1B) which differs in agonist induced desensitization from rMOR1. FEBS Lett. 359, 142-146.

Zorrilla, E.P., 1997. Multiparous species present problems (and possibilities) to developmentalists. Dev. Psychobiol. 30, 141-150.

Zukin, R.S., Eghbali, M., Olive, D., Unterwald, E.M., Tempel, A., 1988. Characterization and visualization of rat and guinea pig brain kappa opioid receptors: evidence for kappa 1 and kappa 2 opioid receptors. Proc. Natl. Acad. Sci. U.S.A. 85, 4061-4065. 
Provided for non-commercial research and education use. Not for reproduction, distribution or commercial use.

\section{Brain Research}
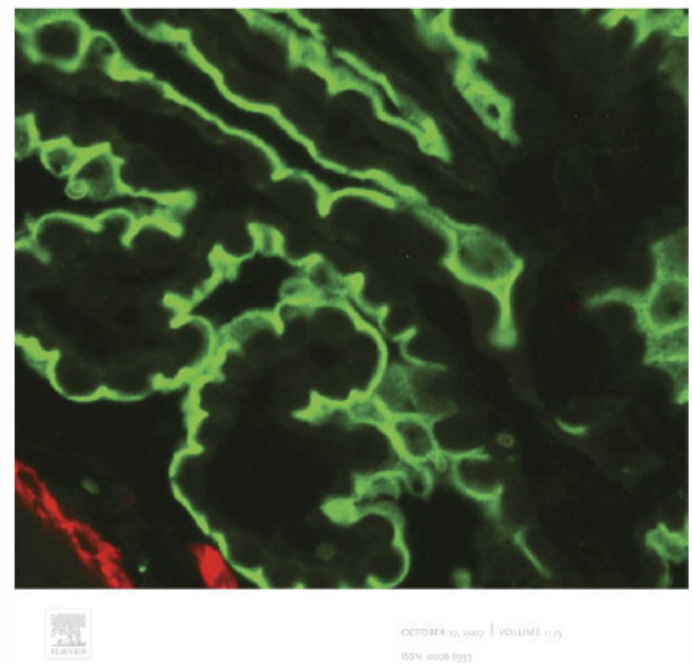

This article was published in an Elsevier journal. The attached copy is furnished to the author for non-commercial research and education use, including for instruction at the author's institution, sharing with colleagues and providing to institution administration.

Other uses, including reproduction and distribution, or selling or licensing copies, or posting to personal, institutional or third party websites are prohibited.

In most cases authors are permitted to post their version of the article (e.g. in Word or Tex form) to their personal website or institutional repository. Authors requiring further information regarding Elsevier's archiving and manuscript policies are encouraged to visit:

http://www.elsevier.com/copyright 
Research Report

\section{Mu opioid receptors are expressed on radial glia but not migrating neuroblasts in the late embryonic mouse brain}

Tim J. Sargeant, Darren J. Day*, Eli M. Mrkusich, Darren F. Foo, John H. Miller

School of Biological Science, Victoria University of Wellington, New Zealand

\begin{tabular}{|c|c|}
\hline A RTICLEINFO & A B S T RACT \\
\hline Artide history: & Mu opioid receptor ligands such as morphine and met-enkephalin are known to modulate \\
\hline Accepted 2 July 2007 & normal brain development by perturbing gliogenesis and inhibiting neuronal proliferation. \\
\hline Available online 22 August 2007 & Surprisingly, the distribution of the mu opioid receptor (MOR) in the embryonic brain, \\
\hline Keywords: & an immunohistochemical approach, we found that MOR protein was expressed in the \\
\hline Ventricular zone & neuroepithelia of the lateral ventricles, third ventricle, and aqueduct within the late \\
\hline Mu opioid receptor & embryonic (E15.5 and E18.5) mouse brain. In contrast to the ventricular neuroepithelia, the \\
\hline Doublecortin & proliferative external granule layer of the embryonic cerebellum did not express MOR \\
\hline GLAST & protein, although the Purkinje cell layer did. Within the ventricular neuroepithelium, GLAST- \\
\hline Proliferation & positive radial glia that incorporate BrdU expressed MOR, while migrating neuroblasts \\
\hline BrdU & (doublecortin-positive) do not. BrdU labeling of proliferating cells showed an anterior to \\
\hline MOR & posterior gradient of proliferation $(P<0.05)$, while an opposing posterior to anterior gradient \\
\hline Cerebral cortex & $\begin{array}{l}\text { of MOR expression }(P<0.05) \text { was found. The localization of MOR immunoreactivity within the } \\
\text { embryonic ventricular neuroepithelia is consistent with a role for opioids in modulating }\end{array}$ \\
\hline
\end{tabular}
neurogenesis.

( 2007 Elsevier B.V. All rights reserved.

\section{Introduction}

The classical opioid receptors include the mu, delta, and kappa subtypes, and their principal endogenous ligands are endorphin and endomorphin (mu), enkephalin (delta), and dynorphin (kappa). The mu opioid receptor (MOR) is activated by its endogenous peptide ligands, as well as exogenous alkaloid opiates such as morphine and heroin (Pasternak, 2004). The biology of the opioid receptors, and in particular MOR, is complicated by the presence of multiple splice variants, alternative promoters, and unique tissue-specific expression patterns of the splice variant isoforms (Abbadie et al., 2000, 2004; Pan et al., 2001; Pan, 2002; Zhang et al., 2006). MOR signaling is generally of an inhibitory nature in that MOR stimulation down-regulates a large number of physiological responses, including cellular proliferation in the developing brain (Kornblum et al., 1987; Zagon and McLaughlin, 1987; Stiene-Martin and Hauser, 1991; Stiene-Martin et al., 1991; Reznikov et al., 1999; Kim et al., 2006). In rats, exposure to morphine in utero causes decreased cortical density of neurons (Seatriz and Hammer, 1993), while studies with mice have shown that MOR activation decreases gliogenesis in the subventricular zone in a developmentally regulated manner (Stiene-Martin et al., 2001). In vitro studies have

\footnotetext{
- Corresponding author. School of Biological Sciences, PO Box 600, Wellington 6140, New Zealand. Fax: +6444635331.

E-mail address: Darren.Day@vuw.ac.nz (D.J. Day).

E-mail address: Darren.Day@vuw.ac.nz (D.J. Day).
Abbreviations: 3V, third ventricle; Aq, aqueduct; BrdU, bromodeoxyuridine; cne, cortical neuroepithelium; DCX, doublecortin; EGL, external granule layer; GLAST, astrocyte-specific glutamate transporter; IR, immunoreactivity; LV, lateral ventricle; MOR, mu opioid receptor; PCNA, proliferating cell nuclear antigen; Str, striatum; VZ, ventricular zone

0006-8993/\$ - see front matter @ 2007 Elsevier B.V. All rights reserved.
}

doi:10.1016/j.brainres. 2007.07.091 


\section{Author's personal copy}

shown that opioids inhibit differentiation and proliferation of neuronal precursor cells isolated from the mouse cerebellum (Hauser et al., 2000). Since MOR ligands are known to inhibit proliferation in the brain, the presence of MOR within germinal zones of the developing brain is anticipated but needs to be unequivocally established, since previous studies have generated conflicting results in regard to the location of MOR MRNA, protein, and ligand binding within this region (Kent et al., 1982; Leslie and Loughlin, 1993; Georges et al., 1998; Leslie et al., 1998; Zhu et al., 1998; Reznikov et al., 1999; Tong et al., 2000).

Radial glia are versatile cells that serve as neuronal progenitors and provide guidance for migrating neurons (Kriegstein and Noctor, 2004). They express a number of immunohistochemical markers in the embryonic CNS, including BLBP, RC2, and glutamate astrocyte-specific transporter (GLAST) (Shibata et al., 1997; Hartfuss et al., 2001; Barry and McDermott, 2005). Migrating neurons are also readily identified by expression of the marker doublecortin (DCX), a microtubule-associated protein that is required for migration and correct lamination in the developing neocortex (Bai et al., 2003). Our study has used staining for GLAST and DCX to identify radial glia and migrating neurons in the lateral ventricle cortical epithelium and the cerebellum. We were particularly interested to determine if MOR was present on migrating neuronal cells, on radial glia, or on both cell types in the developing neocortical germinal zones.

2. Results

2.1. MOR antibody validation

The anti-MOR antibody used in this study was raised against a peptide found within exon 4 in the carboxy-terminus of MOR1. Correct specificity of the MOR-1 antibody was demonstrated

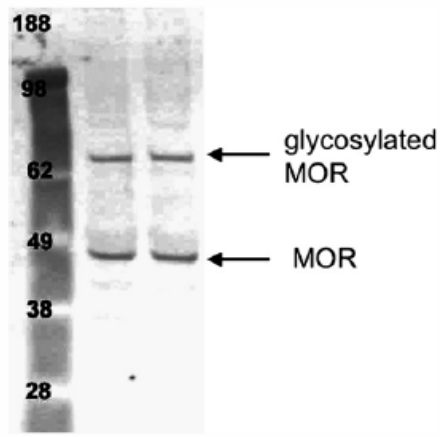

Fig. 1 - Western blotting for MOR. Lysates prepared from the dorsal cortex of E18.5 mouse brains were separated by SDS-PAGE, transferred to a PVDF membrane, and then probed with a rabbit anti-MOR antibody (lanes 2 and 3 ). Immunoreactive bands corresponding to MOR ( $45 \mathrm{kDa}$ ) and the glycosylated MOR $(70 \mathrm{kDa})$ are indicated. Lane 1 contains pre-stained size standards. by obtaining the expected staining pattern in sections of the dorsal horn in mouse spinal cord (data not shown) and also by observing dense staining of embryonic striatum (Figs. 2A and C). Additionally, lysates prepared from cortical dissections corresponding to the same cortical/ventricular regions displayed in Fig. 2 were run on Western blots and stained using the anti-MOR antibody (Fig. 1). A band at approximately 45 $\mathrm{kDa}$, corresponding to the expected size of the unprocessed MOR protein, and a band at $70 \mathrm{kDa}$ corresponding to the glycosylated form of the receptor were observed. These bands and their sizes are as expected, and are in agreement with other studies (Eppler et al., 1993; Liu-Chen et al., 1993 Chalecka-Franaszek et al., 2000; Christoffers et al., 2003; Kivell et al., 2004).

\subsection{MOR staining and PCNA staining}

In the E18.5 brain, MOR immunoreactivity (IR) was found within the ventricular neuroepithelium of the lateral ventricle (LV) (Fig. 2A-D), third ventricle (3V) (Fig. 2E), and aqueduct (Aq) (Fig. 2F). PCNA is a marker for proliferating cells and cells that have recently divided, staining for which allows regions of prolifera tive activity to be identified. PCNAIR can be seen in the nuclei of cells that line the wall of the LV, consistent with previous reports (Ajioka et al., 2006). PCNA-positive cells extend into the cortical neuroepithelial layer that covers the developing striatum (Fig. 2A and C). Importantly, we show in Figs. 2A-D that MORIR is expressed in the same location as the PCNA IR, and that these MOR-positive and the PCNA-positive cells extend along the cortical neuroepithelium (Figs. $2 \mathrm{~B}$ and D). Figs. $2 \mathrm{E}$ and $\mathrm{F}$ show regions of the $3 \mathrm{~V}$ and $\mathrm{Aq}$, respectively, which similar to the LV, express MOR and PCNA at the ventricle wall.

\subsection{MOR and BrdU staining in the ventricular zone}

To examine the relationship between MOR expression and proliferation in detail, a more defined cohort of proliferating cells was labeled with BrdU, as BrdU is only incorporated into cells undergoing DNA synthesis (cells in S-phase of the cell cycle). Dual-staining for BrdU and MOR was examined in the ventricular zone (VZ) of the lateral ventricle in E15.5 and E18.5 mouse brains (Fig. 3). Confocal imaging of the E15.5 brains (Fig. $3 \mathrm{~A})$ showed that most of the BrdU-stained nuclei (green) in the VZ, were MOR-positive (red). Examples of double-labeled cells are indicated by arrows. As not all of the MOR-positive cells were in S-phase, many did not stain for BrdU. At the VZ in the E18.5 brains, fewer BrdU-stained nuclei were detected (Fig. 3B) consistent with the decreased level of corticogenesis at this later developmental stage. Examination of orthogonal views by confocal microscopy (side bars) showed that the cytoplasm surrounding most BrdU-positive nuclei was MOR-positive (arrowheads) even when the $x, y$ view suggested the nuclei were negative for MOR staining as is the case for the cell marked by an asterisk.

\subsection{Proliferation in the developing cerebellum}

The proliferative zone of the developing cerebellum is the external granule layer (EGL), located at the periphery of the cere- 


\section{Author's personal copy}
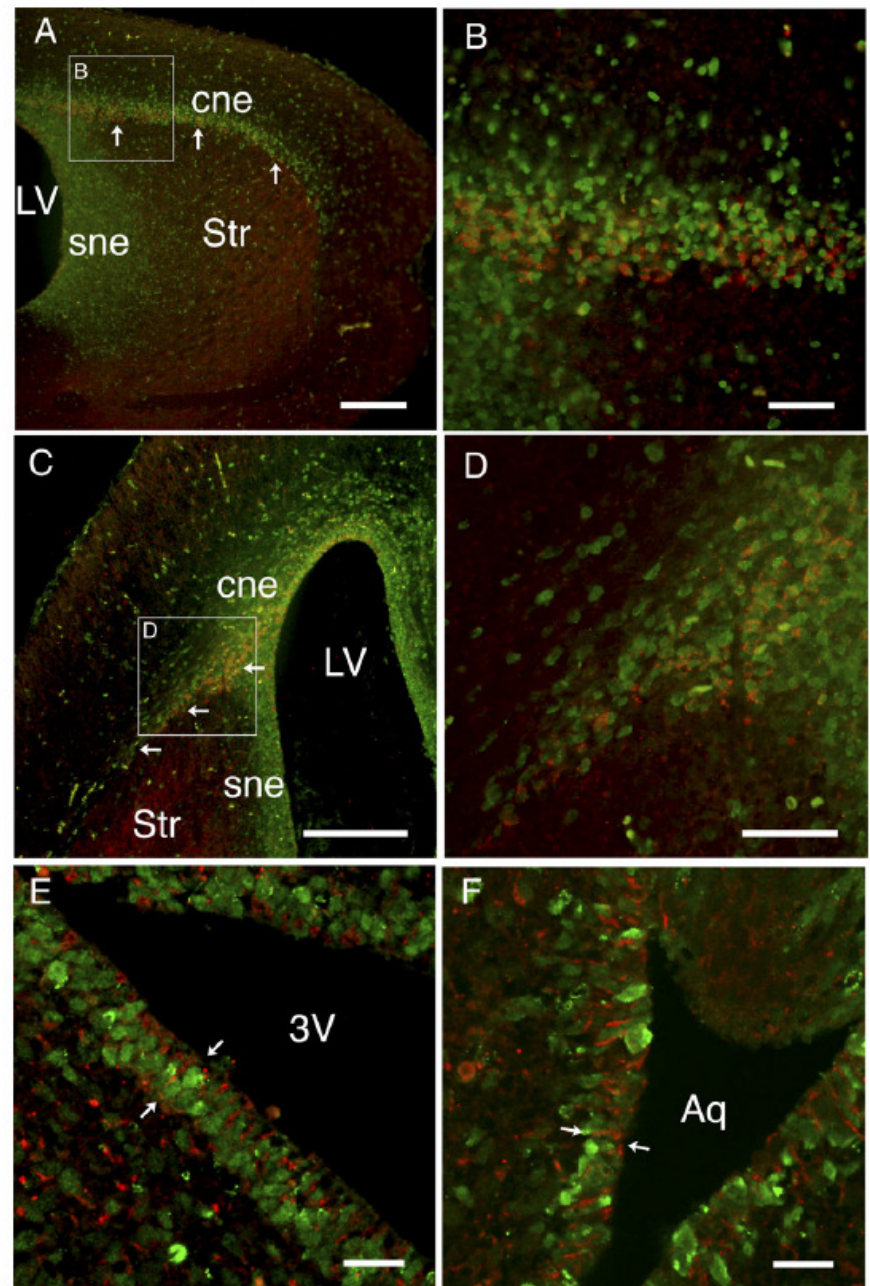

Fig. 2 - MOR and PCNA IR in the E18.5 neuroepithelia of the LV, 3V, and Aq. Panels A and C show MOR IR (red) in coronal and sagittal sections of the LV, respectively. Proliferating cells (PCNA-positive) are stained (green). The cortical neuroepithelium (cne) in panels $\mathrm{A}$ and $\mathrm{C}$ is indicated by the white arrows. Higher magnification images of the boxed regions are presented in panels B and D and show that MOR IR is localized to the same area as PCNA IR. Panel E shows a confocal image of a coronal section of the ventral $3 \mathrm{~V}$ in which MOR IR is tightly associated with a periventricular band of PCNA staining cells (located between the white arrows). Similarly, the proliferative zone of the Aq wall (white arrows, F) contains many PCNA IR nuclei and MOR IR cells. Scale bars: $200 \mu \mathrm{m}$ (A and C), $50 \mu \mathrm{m}$ (B and D) and $20 \mu \mathrm{m}$ (E and F).

bellum and densely packed with proliferating cells that stain strongly for PCNA (Fig. 4A). In contrast to the LV, 3V, and Aq MOR IR was not observed within the proliferative zone of the embryonic cerebellum (Figs. 4B and C) but was present however, in the Purkinje layer. As the high density of cells within the developing cerebellum makes interpretation of immunohistochemical staining of PCNA IR difficult, labeling of cells in S-phase was examined by BrdU incorporation. E18.5, 


\section{Author's personal copy}
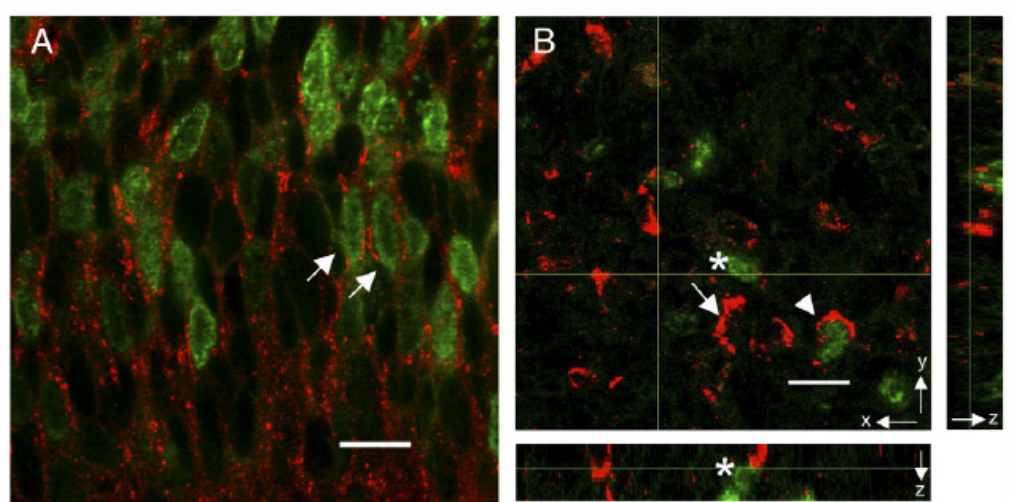

Fig. 3 - MOR and BrdU IR in the E15.5 and E18.5 ventricular zone. Panels A and B show confocal images of the VZ in E15.5 and E18.5 mouse brains, respectively. Panel A shows that most of the Brd-positive nuclei (green) are found in MOR-positive cells (red) in the E15.5 brain. Examples of dual-labeled cells are indicated by arrows. Panel B shows the same region of brain in an E18.5 brain similarly stained. Orthogonal views (z-plane transects at the yellow lines) are presented, and the $x, y$-plane comprising the main panel is indicated within the $z$-planes as green lines. Within the $x, y$-plane, a BrdU-positive/MOR-positive cell (arrowhead) and a BrdU-negative/MOR-positive cell (arrow) are indicated. A cell which appears to stain negative for MOR within the $x, y$ plane but can be seen to be MOR-positive within the $y, z$-plane is indicated by an asterisk ("). Scale bars: $10 \mu \mathrm{m}(\mathrm{A}$ and $\mathrm{B})$.

postnatal day 6 (P6), and $\mathrm{P} 12$ pups were given BrdU $1 \mathrm{~h}$ prior to sacrifice, and the cerebellum stained for MOR IR and BrdU incorporation. Fig. 5A shows that the EGL of theE18.5 cerebellum contains many BrdU positive nuclei (green) that do not stain for MOR (red), while the developing Purkinje/molecular layer stains for MOR but contains few BrdU-positive nuclei. At P6, the Purkinje layer stains intensely for MOR, and the external granular layer contains abundant BrdU-positive nuclei with minimal MOR staining (Fig. 5B). By P12 (Fig. 5C) proliferation is much reduced, but MOR IR in the Purkinje layer is still clearly evident.

\subsection{MOR and GLAST staining}

Radial glia are the neuronal progenitors of the cerebral cortex They stain for GLAST (Figs. 6A-D, green staining) and have processes that extend from the lateral ventricle wall to the pial surface to provide the scaffold on which pyramidal and inter-
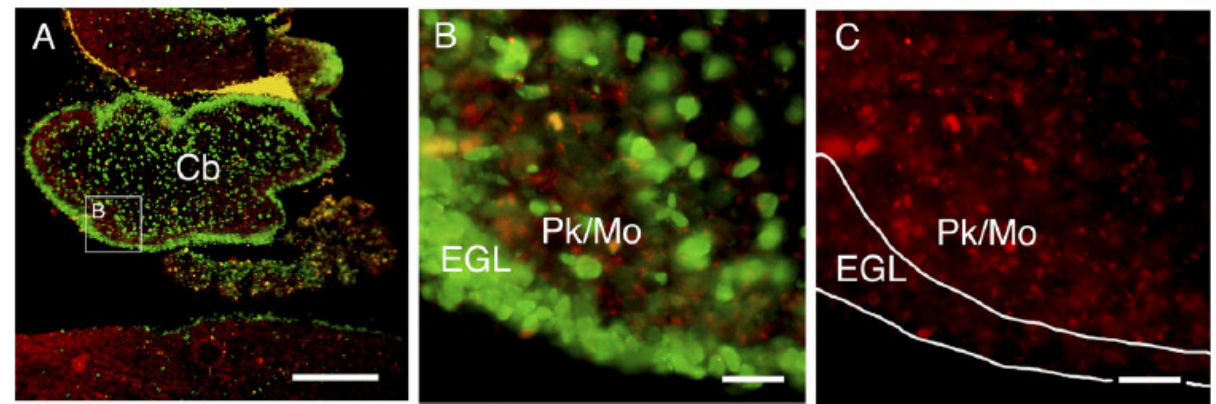

Fig. 4 - PCNA and MOR IR in the E18.5 cerebellum. Panels A and B show both PCNA and MOR IR, while panel C shows only MOR IR. The EGL in panel A is clearly defined by intense PCNA staining (green) at the periphery of the cerebellum (panel A, low power, and panel B, high power). MOR IR (red) is seen within the Purkinje and molecular layers of the cerebellum just beneath the EGL (A and C). Little MOR IR, however, is present within the EGL, which is shown demarcated by the white lines in panel C. Scale bars: $200 \mu \mathrm{m}(A)$ and $20 \mu \mathrm{m}(B$ and C). 


\section{Author's personal copy}
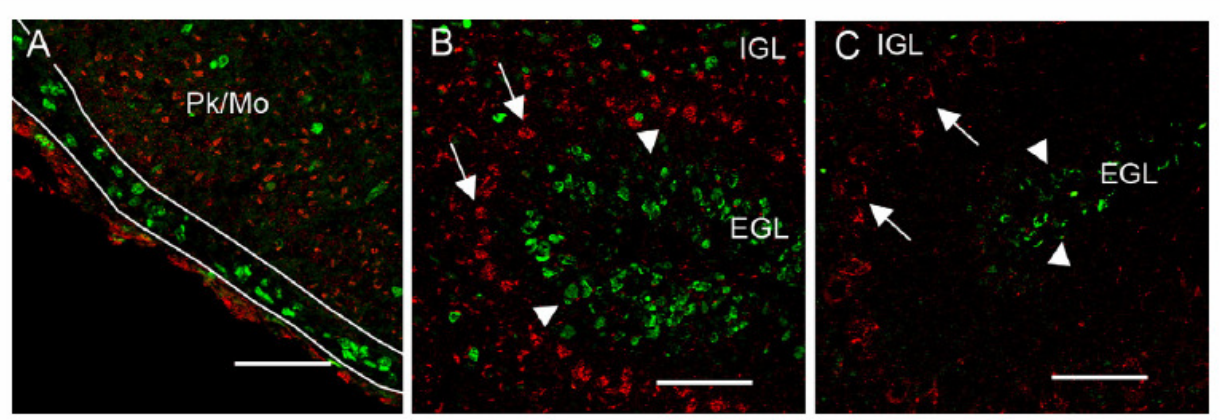

Fig. 5 - Proliferation and MOR IR in the developing cerebellum. The E18.5 EGL is readily identified by its intense staining for BrdU (green, A, demarcated by white lines). MOR IR (red) is seen within the Purkinje and molecular layers (Pk/Mo) of the cerebellum just beneath the EGL. Little MOR IR, however, is present within the EGL. Panel B shows proliferation and MOR expression in the P6 cerebellum similarly stained. Strong MOR IR is present in Purkinje cells (white arrows) of the Purkinje layer, as well as the internal granule layer (IGL). Little MOR IR is seen within the EGL (indicated by the arrowheads) that contains most of the proliferative BrdU-positive cells. Similar staining patterns are seen in the P12 cerebellum (C) with the Purkinje cells being clearly defined as MOR-positive, but with decreased proliferation (BrdU-staining) in the EGL. Scale bars: $50 \mu \mathrm{m}$.

neurons migrate. Higher magnification confocal images of radial glia cell bodies at the LV wall in E15.5 (Fig. 6C) and E18.5 (Fig. 6D) brains show that immunoreactivity for GLAST (green) and MOR (red) is located in the plasma membrane of cells. At E15.5 the VZ contains many cells that are both MOR IR and GLAST IR, although not all GLAST-positive cells stain for MOR. Orthogonal views confirming dual-labeling of a MOR-positive and GLAST-positive cell (indicated by arrows) are shown in Fig. 6C. Similarly, in the E18.5 VZ GLAST-positive cells that stained intensely for MOR (Fig. 6D) are clearly visible. Orthogonal views show that MOR is not evenly distributed within the cell membrane as is evident from cross-sections through the $z$-plane of the cell indicated by an arrow. Using a confocal approach, we found that as for the E15.5 VZ, not al GLAST-positive cells stain for MOR. To determine whether the progenitor cells in the neuroepithelium that overlies the striatum were also MOR and GLAST positive, sections encompassing the brain regions described in Figs. 2A to D were stained. All of the MOR IR cells in this region were also GLASTpositive; however, these MOR IR and GLAST IR cells represent only a small fraction of the GLAST-positive population (Figs. 6E and F). Confocal analysis of the $3 \mathrm{~V}$ and Aq walls (Fig. 7) reveale that the cells at the ventricle walls in these regions were also GLAST-positive (green) with long fibers that radiate perpendicularly (white arrows, Figs. 7A and C). This suggests that these cells radiating out from the $3 \mathrm{~V}$ and $\mathrm{Aq}$ were also radia glia. Figs. 7B and D show that some of these radial glia were also MOR IR (red), as indicated by arrows.

\subsection{MOR and DCX staining}

To determine if the MORIR seen in the cortex was expressed on migrating neurons, sections encompassing the regions described in Fig. 2 were stained for MOR and the neuronal migration-specific marker doublecortin (DCX). Fig. 8A shows
MOR IR (red) and DCX IR (green) around the LV with MOR IR being predominantly localized to the edge of the LV wall (arrowheads). Neurons that have recently migrated toward the pial surface were stained intensely for DCX. Confocal imaging showed that the vast majority of MOR IR cells were not DCX IR. Fig. 8B and C show confocal images of a region of the neuroepithelium equivalent to that presented in Figs. $2 \mathrm{~B}$ and D. As for the LV wall, the MOR IR cells of the cortical neuroepithelium did not express DCX, with MOR IR (red) and DCX IR (green) being mutually exclusive for the majority of cells (Fig. 8B, arrowheads). Fig. $8 \mathrm{C}$ shows a higher-magnification confocal image of two DCX-positive cells (asterisk) in the cortical neuroepithelium. Orthogonal views confirm that these cells do not express MOR, although they are in close proximity to MOR IR processes.

2.7. Gradient of proliferation and MOR expression within the LV cortical neuroepithelium

Previous studies have shown that proliferation is not uniform throughout the cortex, with distinct gradients occurring both medial-lateral and rostral-caudal at early and mid-stages of corticogenesis (Miyama et al, 1997). We therefore wished to examine the topology of proliferation during late corticogenesis (E18.5) in the mouse. Proliferative cells were labeled with BrdU and labeled nuclei counted using a stereological approach on paraffin-embedded sections. BrdU-stained nuclei were much more numerous at the anterior region of the LV than the posterior such that an anterior to posterior gradient of proliferation existed in the E18.5 mouse cortex (Fig. 9). A significant linear regression was fit to the data collected from three mouse embryos $(n=3$ separate litters) $\left(R^{2}=0.82, P<0.05\right)$. The density of MOR IR cells was determined by counting fluorescently stained cells using a photomicroscope Cell counts for MOR IR were expressed in Fig. 9 as the number of cells per unit area. The density of MOR IR 

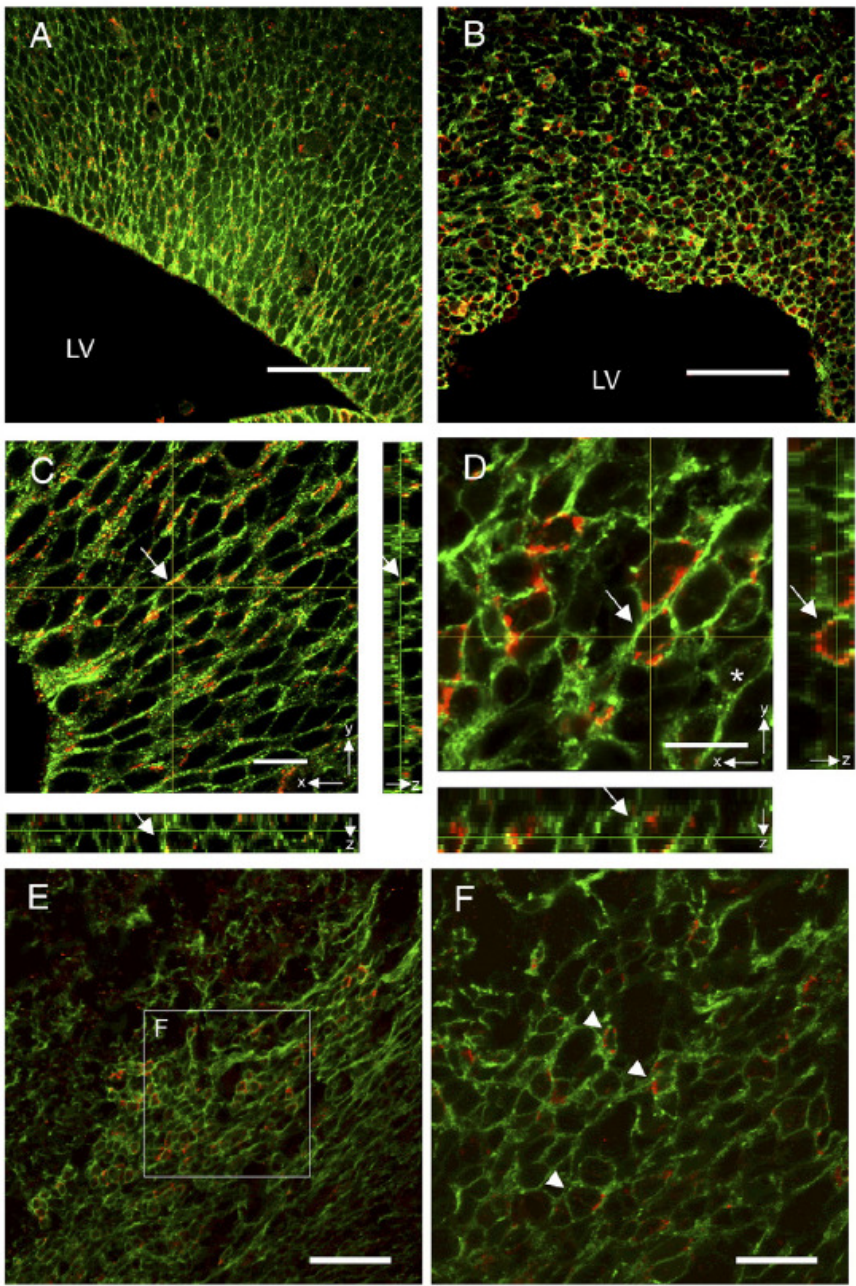

Fig. 6 - MOR and GLAST IR in the E15.5 and E18.5 LV neuroepithelium. Panels A and B show low power images of the E15.5 and E18.5 VZ, respectively. Both panels show MOR IR (red) in the same region as GLAST staining (green). Panel C shows a higher magnification image of the E15.5 VZ in which MOR IR (red) and GLAST IR (green) can be seen within the same cell. Orthogonal views (z-plane) of the double-labeled cell (indicated by the arrow) are presented in the side bars. Similarly, panel D shows a higher power image of the E18.5 ventricular zone in which double-labeling for MOR and GLAST is more apparent. Nearly all the MOR-positive cells examined stained for GLAST, but many GLAST-positive cells did not stain or MOR. A GLAST-positive/MOR-negative cell is marked with an asterisk, and orthogonal views through a MOR-positive/GLAST positive cell (arrows) are presented in the side bars. Panels E and F show similarly stained regions of cortical neuroepithelium equivalent to that in Fig. 2D) from E18.5 brains in which MOR IR and GLAST IR are found within the same cell (arrowheads). Scale bars: $50 \mu \mathrm{m}(\mathrm{A}, \mathrm{B}$ and $\mathrm{E}), 20 \mu \mathrm{m}(\mathrm{F})$ and $10 \mu \mathrm{m}$ (C and D). 


\section{Author's personal copy}
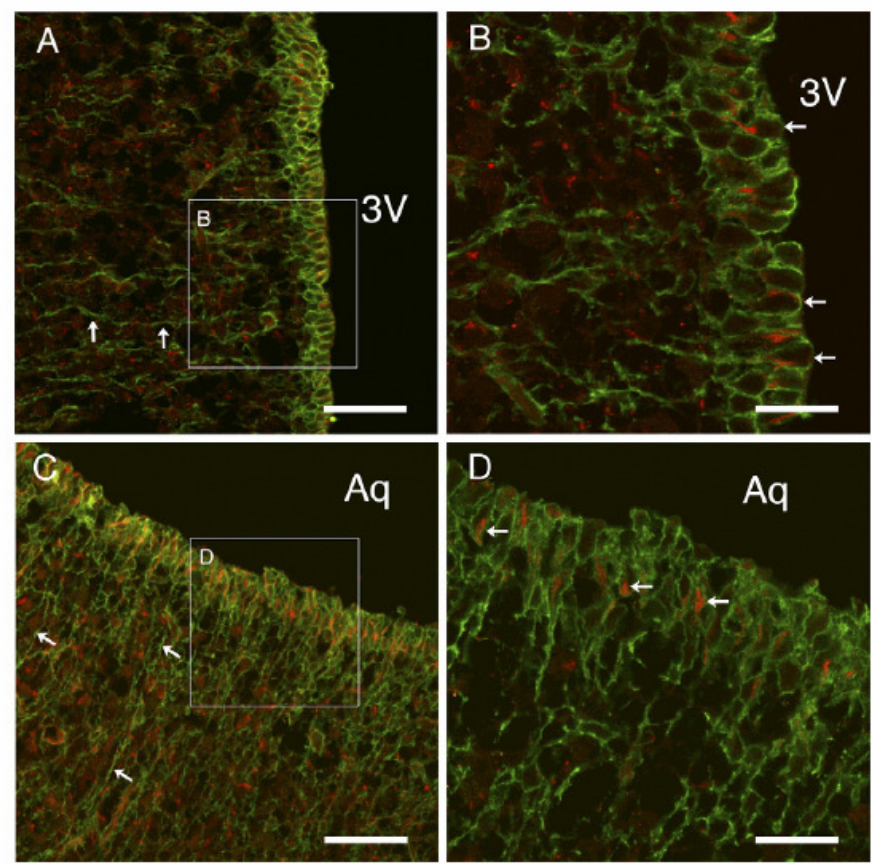

Fig. 7 - MOR and GLAST IR in the neuroepithelia of the 3V and Aq in the E18.5 mouse. Panel A shows intense GLAST staining (green) at the wall of the 3V. GLAST IR processes that extend perpendicular from the ventricular wall can be clearly seen (white arrows). MOR IR (red) is also located around the 3V (A and B). White arrows in panel B identify some of the cells that stain for both MOR and GLAST. Panel C shows MOR and GLAST IR at the wall of the Aq. Similarly, GLAST IR processes extend perpendicular from the wall of the Aq (C, white arrows) with many of the GLAST IR cells also expressing MOR (D, white arrows). Scale bars: $50 \mu \mathrm{m}$ (A and C) and $20 \mu \mathrm{m}(\mathrm{B}$ and $\mathrm{D})$.

followed the opposite trend to that for BrdU labeling, with MOR expression decreasing in a posterior to anterior manner, with the greatest density of MOR-positive cells in the posterior sections. A significant linear regression was fit to the data collected from three mouse embryos $(n=3$ separate litters $)\left(R^{2}=0.91, P<0.05\right)$.

3.

Discussion

The present study has demonstrated that MOR is expressed within the neuroepithelium of the lateral ventricles, the third ventricle, and the aqueduct of the E15.5 and E18.5 mouse brain, and that cells expressing MOR within the lateral ventricle incorporate BrdU, and hence are proliferative. MOR IR cells within ventricular neuroepithelia were also GLAST IR indicating that they are radial glia. MOR IR cells in proliferating regions were not DCX IR, suggesting that the MOR-positive cells within neuroepithelium are radial glial progenitor cells and not migrating daughter neuroblasts. The same staining pattern was seen at all the ventricular surfaces examined.
In contrast to the epithelia lining the ventricular walls, the proliferative zone (EGL) of the developing cerebellum lacked MOR immunoreactivity in the E18.5 brain. The cerebellum is a late developing structure and is extremely immature in the late embryonic mouse (Goldowitz and Hamre, 1998). We and others have previously reported that the rodent cerebellum expresses MOR mRNA and protein, predominantly in the Purkinje layer and the deep cerebellar nuclei (Mrkusich et al, 2004; Xu et al., 2006). Given the relative immaturity of the cerebellum at birth and its continued development up to the third postnatal week (Goldowitz and Hamre, 1998), we reasoned that MOR expression may increase after E18.5. This was, however, not the case as the P6 and P12 cerebellum showed strong MOR staining of Purkinje cells, as in the adult, but replicating (BrdU-positive) cells still lacked MOR expression in the postnatal brain. These data suggest a different role for MOR signaling in the developing cerebellum than that in the ventricular neuroepithelia. The data also corroborate our previous report that MOR mRNA is expressed in Purkinje cells of the developing cerebellum (Mrkusich et al., 2004). 


\section{Author's personal copy}
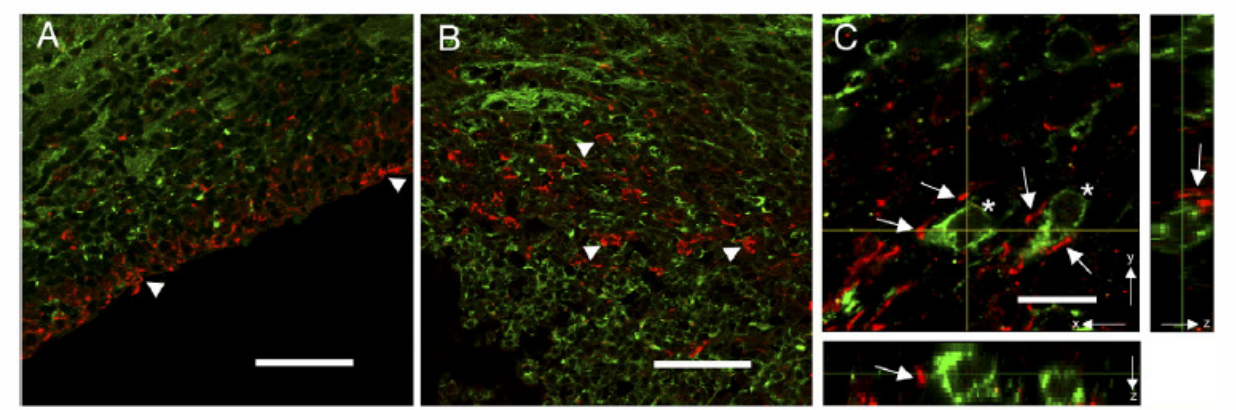

Fig. 8 - MOR and DCX IR in the E18.5 neocortex. Panel A shows a region of VZ at the lateral ventricle wall in an E18.5 brain stained for MOR (red) and DCX (green). MOR IR is located mainly within the VZ at the LV wall, with few dual-labeled MOR IR/DCX IR cells apparent. Panel B shows an equivalent region of cortical neuroepithelium to that presented in Fig. 2D. Most of the MOR IR cells do not express DCX (arrowheads). Orthogonal views are presented in the side bars of MOR-positive cells that confirm the absence of DCX IR within these cells. Panel C shows a higher magnification image of cells presented in panel B. Two DCX positive cells in the $x, y$-plane are indicated with an asterisk, and orthogonal views in the $z$-plane are presented in the side bars. Cytoplasmic staining for DCX (green) is clearly visible, and MOR IR (red) processes can be seen contacting the cell bodies (arrowheads). The orthogonal views confirm that the MOR IR seen adjacent to the cell bodies is not within the DCX-positive cell. Scale bars: $50 \mu \mathrm{m}$ (A and B) and $10 \mu \mathrm{m}(\mathrm{B})$.

The existence of an anterior to posterior gradient of proliferation within the lateral ventricle and an inverse gradient to MOR expression is consistent with the idea of MOR activation being anti-proliferative. It is, however, speculative to suggest that MOR signaling maintains or regulates the gradient of proliferation in vivo as there is still little direct supporting evidence to indicate that opioid ligands have an anti-proliferative effect in vivo via MOR signaling (Reznikov et al., 1999; Eisch et al., 2000; Stiene-Martin et al., 2001; Mandyam et al., 2004). The data we have presented in this study does, however, place expression of the receptor on the appropriate cells for modulation of corticogenesis. It is interesting to note that morphine exposure through the period E12 to P6 in the rat decreased cortical neuronal density (Seatriz and Hammer, 1993).

It should be noted that the antibody used in this study (Chemicon, rabbit anti-MOR, AB5511) was raised against exon 4 of MOR. Exon 4 containing splice variants of the mu opioid receptor includes MOR-1, as well as the exon-11 containing splice variants MOR-1H to MOR-1L (Abbadie et al., 2004). However, this antibody will not recognize the rodent carboxy terminus MOR-1 splice variants such as MOR-1A to MOR-1F. As these MOR-1 carboxy terminus splice variants differ in their responses to endogenous opioid peptides as well as exogenous opiates (Bolan et al., 2004; Pasternak et al., 2004), future work needs to characterize the embryonic expression patterns of these variant receptors in relation to MOR-1 expression. We have previously described detection of mRNA coding MOR in the developing rat cerebellum (Mrkusich et al. 2004). This study used riboprobes that detected the $5^{\prime}$ UTR of MOR-1 mRNA. Using the anti-MOR antibody detailed in this present study, we found good correlation between MOR-1 mRNA and protein expression in all regions of the cerebellum apart from the EGL. As others have shown differential immunohistochemical localization for carboxy terminus splice variants in the adult rat CNS (Abbadie et al, 2000), it

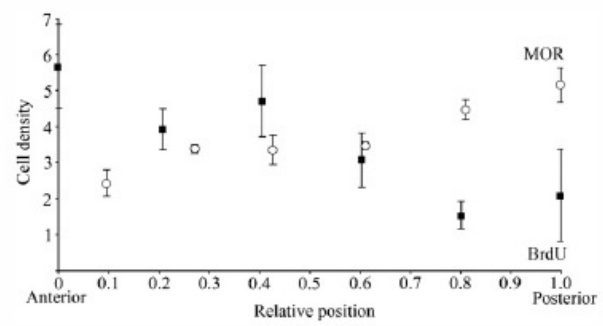

Fig. 9 - Gradients of proliferation and MOR expression in the ventricular zone of the LV in E18.5 mice. BrdU incorporation decreased in an anterior to posterior direction $(P<0.05)$ (black squares), while the number of MOR IR cells (empty circles) increased anterior to posterior $(P<0.05)$. Staining for BrdU incorporation was performed on paraffin-embedded sections and counted stereologically, while MOR IHC was performed on fixed, then cryopreserved, $20 \mu \mathrm{m}$ sections using non-stereological methods. To alleviate the effects of tissue shrinkage that occurs with paraffin embedding, the relative distance from the first anterior appearance of the LV (set arbitrarily at 0 units) to its disappearance posteriorly (1 unit) is plotted along the $x$-axis. The $y$-axis represents the density of BrdU-stained nuclei $\left(\mathrm{mm}^{-3} \times 10^{5}\right)$ and MOR-stained cells $\left(\mathrm{mm}^{-2} \times 10^{3}\right)$. Values represent the mean \pm S.E.M. $(n=3$ separate litters). $P$ values were determined by regression analysis. 


\section{Author's personal copy}

is possible that the discordance between protein expression and mRNA localization in the EGL may be attributable to expression of a splice variant lacking exon 4 , or alternatively it may simply reflect the lower resolution of mRNA in situ hybridization studies being further confounded by the high cell density in the developing cerebellum reducing the signalto-noise ratio.

One of the few studies to look in detail at MOR mRNA distributions in the germinal zones of the embryonic CNS used in situ hybridization to show that MOR MRNA was absent in all the CNS germinal centres of the embryonic mouse (Zhu et al., 1998). A similar conclusion was reached for the embryonic rat CNS (Leslie et al., 1998). In another in situ hybridization study, however, MOR MRNA was found in or near many of the germinal zones early in the fetal rat brain (E12) (Tong et al., 2000). The study by Tong et al. (2000) is discordant with that of Zhu et al. (1998) and Leslie et al (1998), but concurs with a study by Reznikov et al. (1999) and our own. In support of our results, ligand binding measurements locate MOR protein within the proliferative zones of the embryonic forebrain in rats from E16 onwards (Leslie and Loughlin, 1993). It was suggested in this study that the reason for the differences between mRNA in situ hybridization and MOR ligand binding was the superior resolution of the mRNA in situ hybridization technique. However, the immunohistochemical data we present here and that of Reznikov et al. (1999) have clearly demonstrated that MOR protein is expressed within embryonic neuroepithelia. In support of our studies, preliminary in situ hybridization experiments conducted in the embryonic rat (Supplementary Fig. 1) have localized MOR mRNA to the VZ using a riboprobe targeting the 5'UTR of MOR-1 mRNA (Mrkusich et al. 2004).

Little information is available on the presence of MOR ligands within germinal zones of the embryonic brain, although a few investigators have shown that preproenkephalin mRNA is abundantly expressed in the embryonic lateral ventricular neuroepithelium (Zagon et al., 1994; Leslie et al., 1998). Some inconsistencies exist, however, since Zagon et al. (1994) report widespread expression of preproenkephalin mRNA within proliferative zones of the fetal rat brain; whereas, Leslie et al. (1998) report that preproenkephalin mRNA is only expressed within the ventricular neuroepithelium of the lateral ventricles. Both studies, at least, provide good evidence that opioidergic signaling exists within the developing embryonic brain from midgestation (E12) onwards. No one has looked for other endogenous MOR ligands, such as endorphin and endomorphin, within embryonic germinal zones. This work needs to be carried out.

In the present study, MOR expression in proliferative neuroepithelia of the developing mouse brain was observed only on replicating radial glia (BrdU-positive, GLAST-positive cells) around the ventricular neuroepithelia, but not on newborn migrating neurons (DCX-positive cells). As there is increasing evidence in the literature to support the hypothesis that MOR signaling modulates cell cycle progression in vitro, the possibility that MOR modulates cell cycle progression in the developing embryonic brain warrants further investigation.

\section{Experimental procedures}

4.1. Tissue preparation

All experiments involving animals were approved by the Victoria University Animal Ethics Committee. Embryonic brains for each experiment were obtained from at least three separate litters of C57B6/J mice. Animals were given water and food ad libitum and maintained on a $12 \mathrm{~h}: 12 \mathrm{~h}$ light-dark cycle. Brains were rapidly dissected from euthanized animals and fixed in ice-cold $4 \%$ paraformaldehyde in PBS for $9 \mathrm{~h}$. Brains were either processed for paraffin embedding through a graded ethanol/xylene series or they were cryoprotected in $30 \%$ sucrose overnight and stored at $-80^{\circ} \mathrm{C}$ until sectioned. Tissue was sectioned at $20 \mu \mathrm{m}$ thickness using an 820 series microtome (American Optical Co., Buffalo, NY) for paraffin embedded samples, while frozen-fixed tissue was mounted in Jung Tissue Freezing Medium (Leica Instruments, Nussloch, Germany) and sectioned using an HM 500 OM series cryostat microtome (Microm, Heidelberg, Germany). Sections were mounted on Superfrost plus slides (Esco, Erie Scientific, Portsmouth, NH).

\subsection{Bromodeoxyuridine incorporation}

Bromodeoxyuridine (BrdU) (Sigma) was administered as a single $50 \mathrm{mg} / \mathrm{kg}$ intraperitoneal dose. Pregnant dams (E15.5 and E18.5) were sacrificed $3 \mathrm{~h}$ after BrdU treatment, while P6 and $\mathrm{P} 12$ pups were sacrificed $1 \mathrm{~h}$ after BrdU administration.

\subsection{Immunohistochemistry}

Frozen sections were air-dried and permeabilized at room temperature in PBS containing $0.05 \%$ Triton $\mathrm{X}-100$ for $15 \mathrm{~min}$ and blocked for $1 \mathrm{~h}$ at room temperature in PBS containing 2\% BSA and $10 \%$ goat serum. Sections were incubated overnight at $4{ }^{\circ} \mathrm{C}$ with rabbit anti-MOR antibodies (1:1000 dilution, AB5511 Chemicon, Temecula, CA) and either guinea pig anti-DCX antibodies (1:1000 dilution, AB5910, Chemicon) or guinea-pig anti-GLAST antibodies (1:1000 dilution, AB1782, Chemicon). All antibodies were diluted in PBS containing $2 \%$ BSA and $10 \%$ goat serum. Sections were washed four times for $10 \mathrm{~min}$ each in PBS containing $0.1 \%$ Tween. Sections were then incubated with the appropriate secondary antibodies, goat anti-rabbitAlexa 555 (1:500 dilution, A21429, Invitrogen, Molecular Probes, Eugene, OR), and goat anti-guinea pig IgG-Alexa 488 (1:500 dilution, $\mathrm{A} 11073$, Invitrogen) for $1 \mathrm{~h}$ at room temperature. Sections were washed as for the primary antibody.

Paraffin-embedded sections were de-paraffinized in xylene and rehydrated through a graded ethanol series. Antigens were retrieved at $100^{\circ} \mathrm{C}$ in $10 \mathrm{mM}$ sodium citrate, $\mathrm{pH} 6.0$ for $10 \mathrm{~min}$. The BrdU epitope was either retrieved with sodium citrate as above for paraffin embedded samples or for frozen sections by incubating for $10 \mathrm{~min}$ at $37^{\circ} \mathrm{C}$ in $40 \mathrm{mM}$ Tris- $\mathrm{HCl}$ buffer $\mathrm{pH} 8.0$ containing $10 \mathrm{mM} \mathrm{NaCl}, 10 \mathrm{mM} \mathrm{CaCl}_{2}, 6 \mathrm{mM}$ $\mathrm{MgCl}_{2}$, and $250 \mu \mathrm{g} / \mathrm{ml}$ DNAse I (Roche). Tissue sections were then processed as for the frozen sections with specific primary antibodies (mouse anti-PCNA, 1:50 dilution, MAB424, Chemicon; mouse anti-BrdU, 1:100, A21301 MP, Invitrogen) or MOR as described above. Bound primary antibodies were visualized by 


\section{Author's personal copy}

staining with appropriate secondary antibodies. BrdU was visualized by diaminobenzidine (DAB) staining using a Vectastain Elite ABC Kit (PK-6100, Vector Laboratories, Burlingame, CA) according to the manufacturer's instructions or with a streptavidin-conjugated Alexa 488 secondary antibody (S-32354, 1:1000 dilution, Invitrogen).

Slides were mounted in either DPX or Prolong Gold Antifade reagent (P36934, Invitrogen) as appropriate. Controls in which the primary antibody was omitted showed no specific staining when compared with tissue in which the primary antibody was included.

\subsection{Image analysis}

Sections were viewed either by confocal microscopy or with an Olympus AX70 photomicroscope (Olympus Optical Co., Hamburg, Germany), equipped with appropriate narrowband fluorescence filters. Images from the photomicroscope were captured using an Olympus DP70 CCD camera. Confocal microscopy was performed using an Olympus FluoView FV1000. Photomicroscope and confocal images were overlaid and background corrected for presentation purposes, using ImageJ v1.34s (NIH, USA) and Adobe Photoshop CS3 software.

\subsection{Cell counting}

Cells were counted using random-systematic sampling with an unbiased counting frame (Bonthius et al., 2004). Cell densities within the neocortical neuroepithelium were counted in coronal sections beginning with the appearance of the anterior portion of the LV until its disappearance in more caudal sections. Cell counting was performed on neuroepithelia of the dorsal surface of the lateral ventricle.

\section{Acknowledgments}

The authors would like to thank Dr. Eli Mrkusich for contributions to this study, Tertiary Education Commission (NZ) for the award of a Bright Future PhD Scholarship to TJS, and financial support from the Wellington Medical Research Foundation Inc.

\section{Appendix A. Supplementary data}

Supplementary data associated with this article can be found, in the online version, at doi:10.1016/j.brainres.2007.07.091.

\section{REFERENCES}

Abbadie, C., Pan, Y.X., Drake, C.T., Pasternak, G.W., 2000. Comparative immunohistochemical distributions of carboxy terminus epitopes from the mu-opioid receptor splice variants MOR-1D, MOR-1 and MOR-1C in the mouse and rat CNS. Neuroscience 100, 141-153.

Abbadie, C., Pan, Y.X., Pasternak, G.W., 2004. Immunohistochemical study of the expression of exon 11 -containing mu opioid receptor variants in mouse brain. Neuroscience 127, 419-430.
Ajioka, I., Maeda, T., Nakajima, K., 2006. Identification of ventricular-side enriched molecules regulated in a stage-dependent manner during cerebral cortical development. Eur. J. Neurosci. 23, 296-308.

Bai, J., Ramos, R.L., Ackman, J.B., Thomas, A.M., Lee, R.V., LoTurco, J.J., 2003. RNAi reveals doublecortin is required for radial migration in rat neocortex Nat. Neurosci. 6, 1277-1283.

Bolan, E.A., Pan, Y-X., Pasternak, G.W., 2004. Functional analysis of MOR-1 splice variants of the mouse mu opioid receptor gene Oprm. Synapse 51, 11-18.

Banry, D., McDermott, K., 2005. Differentiation of radial glia from radial precursor cells and transformation into astrocytes in the developing rat spinal cord. Glia 50, 187-197.

Bonthius, D.J., McKim, R., Koele, L., Harb, H., Karacay, B., Mahoney, J., Pantazis, N.J., 2004. Use of frozen sections to determine
nof., neuronal number in the murine hippocampus and neocortex using optical dissector and optical fractionator. Brain Res. Protoc. 14, 45-57.

Chalecka-Franaszek, E., Weems, H.B., Crowder, A.T., Cox, B.M. Cote, T.E., 2000. Immunoprecipitation of high-affinity, guanine nucleotide-sensitive solubilized mu opioid receptors from the rat brain: coimmunoprecipitation of the $G$ proteins $\mathrm{G}_{\mathrm{a1}}, \mathrm{G}_{\mathrm{c} i 1}$, and $\mathrm{G}_{\mathrm{\alpha} 3 \mathrm{i}}$. J. Neurochem. 74, 1068-1078.

Christoffers, K.H., Li, H., Keenan, S.M., Howells, R.D., 2003. Purification and mass spectrometric analysis of the mu opioid receptor. Brain Res. Mol. Brain Res. 118, 119-131.

Eisch, A.J., Barrot, M., Schad, C.A., Self, D.W., Nestler, E.J., 2000. Opiates inhibit neurogenesis in the adult rat hippocampus. Proc. Natl. Acad. Sci. U.S.A. 97, 7579-7584.

Eppler, C.M., Hulmes, J.D., Wang, J-B., Johnson, B., Corbett, M., Luthin, D.R., Uhl, G.R., Linden, J., 1993. Purification and partial amino acid sequence of a mu opioid receptor from rat brain. J. Biol. Chem. 268, 26447-26451.

Georges, F., Normand, E., Bloch, B., LeMoine, C., 1998. Opioid receptor gene expression in the rat brain during ontogeny, with
renter special reference to the mesostriatal system: an in situ hybridization study. Dev. Brain Res. 109, 187-199.

Goldowitz, D., Hamre, K., 1998. The cells and molecules that make a cerebellum. Trends Neurosci. 21, 375-382.

Hartfuss, E., Galli, R., Heins, N., Gotz, M., 2001. Characterization of CNS precursor subtypes and radial glia. Dev. Biol. 229, 15-30.

Hauser, K.F., Houdi, A.A., Turbek, C.S., Elde, R.P., Maxson, W., 2000 Opioids intrinsically inhibit the genesis of mouse cerebellar granule neuron precursors in vitro: differential impact of granule neuron precursors in vitro: differential impact of
mu and delta receptor activation on proliferation and neurite $\mathrm{mu}$ and delta receptor activation on prolife
elongation. Eur. J. Neurosci. 12, 1281-1293.

Kent, J.L., Pert, C.B., Herkenham, M., 1982. Ontogeny of opiate receptors in rat forebrain: visualization by in vitro autoradiography. Dev. Brain Res. 2, 487-504.

Kim, E., Clark, A.L., Kiss, A., Hahn, J.W., Wesselschmidt, R., Coscia, C.J., Belcheva, M.M., 2006. Mu and kappa-opioids induce the differentiation of embryonic stem cells to neural progenitors. J. Biol. Chem. 281, 33749-33760.

Kivell, B.M., Day, D.J., McDonald, F.J., Miller, J.H., 2004 Developmental expression of mu and delta opioid receptors in the rat brainstem: evidence for a postnatal switch in mu isoform expression. Dev. Brain Res. 148, 185-196.

Kornblum, H.I., Loughlin, S.E., Leslie, F.M., 1987. Effects of morphine on DNA synthesis in neonatal rat brain. Dev. Brain Res. 31, 45-52.

Kriegstein, A.R., Noctor, S.C., 2004. Patterns of neuronal migration in the embryonic cortex. Trends Neurosci. 27, 392-399.

Leslie, F., Loughlin, S., 1993. Ontogeny and plasticity of opioid systems. In: Hammer, R.P. (Ed.), The Neurobiology of Opiates. CRC Press, Boca Raton, FL, pp. 85-123.

Leslie, F.M., Chen, Y., Winzer-Serhan, U.H., 1998. Opioid receptor and peptide mRNA expression in proliferative 


\section{Author's personal copy}

zones of fetal rat central nervous system. Can. J. Physiol. Pharm. 76, 284-293.

Liu-Chen, L.Y., Chen, C., Phillips, C.A., 1993. Beta- $\left.\left.\right|^{\beta} \mathrm{H}\right]$

funaltrexamine-labeled mu-opioid receptors: species variation in molecular mass and glycosylation by complex-type, $\mathrm{N}$-linked oligosaccharides. Mol. Pharmacol. 44, 749-756.

Mandyam, C.D., Norris, R.D., Eisch, A.J., 2004. Chronic morphine induces premature mitosis of proliferating cells in the adult mouse subgranular zone. J. Neurosci. Res. 76 783-794.

Miyama, S., Takahashi, T., Nowakowski, R.S., Caviness, V.S., 1997. A gradient in the duration of the G1 phase in the murine neocortical proliferative epithelium. Cereb. Cortex 7 . murine ne.689.

Mrkusich, E.M., Kivell, B.M., Miller, J.H., Day, D.J., 2004. Abundant expression of mu and delta opioid receptor mRNA and protein in the cerebellum of the fetal, neonatal, and adult rat Dev. Brain Res. 148, 213-222.

Pan, Y.X., 2002. Identification and characterization of a novel promoter of the mouse opioid receptor gene (Oprm) that generates eight splice variants. Gene 295, 97-108.

Pan, Y.X., Xu, J., Mahurter, L., Bolan, E., Xu, M., Pasternak, G.W., 2001. Generation of the mu opioid receptor (MOR-1) protein by three new splice variants of the Opm gene. Proc. Natl. Acad. Sci. U.S.A. 98, 14084-14089.

Pasternak, G.W., 2004. Multiple opiate receptors: de ja' vu all over again. Neuropharmacology $47,312-323$

Pasternak, D.A., Pan, L., Xu, J., Yu, R., Xu, M-M., Pastemak, G. W., Pan, Y-X., 2004. Identification of three new alternatively spliced variants of the rat mu opioid receptor gene: dissociation of affinity and efficacy. J. Neurochem. 91 881-890.

Reznikov, K., Hauser, K.F., Nazarevskaja, G., Trunova, Y., Derjabin, V., Bakalkin, G., 1999. Opioids modulate cell division in the germinal zone of the late embryonic neocortex. Eur.J. Neurosci. 11, 2711-2719.

Seatriz,J.V., Hammer, R.P., 1993. Effects of opiates on neurona development in the ratcerebral cortex. Brain Res. Bull. 30, 523-527.
Shibata, T., Yamada, K., Watanabe, M., Ikenaka, K., Wada, K. Tanaka, K., Inoue, Y., 1997. Glutamate transporter GLAST is expressed in the radial glia-astrocyte lineage of developing mouse spinal cord. J. Neurosci. 17, 9212-9219.

Stiene-Martin, A., Hauser, K.F., 1991. Glial growth is regulated by agonists selective for multiple opioid receptor types in vitro. J. Neurosci. Res. 29, 538-548.

Stiene-Martin, A., Gurwell, J.A., Hauser, K.F., 1991. Morphine alters astrocyte growth in primary cultures of mouse glial cells: evidence for a direct effect of opiates on neural maturation. Dev. Brain Res. 60, 1-7.

Stiene-Martin, A., Knapp, P.E., Martin, K., Gurwell, J.A., Ryan, S., Thornton, S.R., Smith, F.L., Hauser, K.F., 2001. Opioid system diversity in developing neurons, astroglia and oligodendroglia in the subventricular zone and striatum: impact on gliogenesis in vivo. Glia $36,78-88$.

Tong, Y., Chabot, J.G., Shen, S.H., O'Dowd, B.F., George, S.R., Quirion, R., 2000. Ontogenic profile of the expression of the mu opioid receptor gene in the rat telencephalon and diencephalon: an in situ hybridization study. J. Chem. Neuroanat. 18, 209-222.

Xu, J., Xu, M., Pan, Y.X., 2006. Characterizing exons 11 and 1 promoters of the mu opioid receptor (Oprm) gene in transgenic mice. BMC Mol. Biol. 7, 41-56

Zagon, I.S., McLaughlin, P.J., 1987. Endogenous opioid systems regulate cell proliferation in the developing rat brain. Brain Res. $412,68-72$.

Zagon, I.S., Isayama, T., McLaughlin, P.J., 1994. Preproenkephalin mRNA expression in the developing and adult rat brain. Dev. Brain Res. 21, 85-98.

Zhang, Y., Pana, Y.X., Kolesnikovb, Y., Pasternak, G.W., 2006. Immunohistochemical labelling of the mu opioid receptor carboxy terminal splice variant mMOR-1B4 in the mouse central nervous system. Brain Res. 1099, $33-43$.

Zhu, Y., Hsu, M.S., Pintar, J.E., 1998. Developmental expression of the mu, kappa and delta opioid receptor mRNAs in mouse. J. Neurosci. 18, 2538-2549. 


\section{Acute in utero morphine exposure slows $\mathrm{G}_{2} / \mathrm{M}$ phase transition in radial glial and basal progenitor cells in the dorsal telencephalon of the E15.5 embryonic mouse}

Tim J. Sargeant, Darren J. Day, John H. Miller and Ryan W. J. Steel

School of Biological Sciences, PO Box 600, Wellington 6140, New Zealand

Keywords: interkinetic nuclear migration, opioid receptor, radial glia, ventricular zone

Abstract

The antiproliferative effects of opiate exposure on neurogenesis in vitro have been well documented, but the effects of opiates on brain development in vivo are less well understood. We have recently shown that mu opioid receptors are expressed on radial glia of the lateral ventricle, the neuronal and glial progenitor cells of the developing cortex. In the present study we show that in vivo morphine treatment of the $\mathrm{E} 15.5$ mouse increases the length of the $\mathrm{G}_{2} / \mathrm{M}$ phase of the radial glial cell cycle in the dorsal telencephalon, as well as slows interkinetic nuclear migration of radial glial nuclei from the basal ventricular zone to the apical surface. A prolonged $\mathrm{G}_{2} / \mathrm{M}$ phase was also observed in basal progenitor cells. Although morphine exposure altered the duration of the cell cycle for progenitor cells in the embryonic telencephalon, it did not affect whether the progenitors remained proliferative and re-entered the S phase, or whether they exited the cell cycle and became quiescent. In addition, morphine treatment did not change the proportion of basal to apical mitoses. These findings indicate that opioid signalling plays a role in cell cycle progression of both radial glia and basal progenitor cells in vivo in the developing cerebral cortex.

Introduction

The murine cerebral cortex forms during a burst of cellular proliferation in the late embryo [embryonic day (E)12-E17; Smart, 1973; Takahashi et al., 1996; Caviness et al. 2003]. Neurons of the cerebral cortex migrate into the embryonic cortical plate from the ventricular zone (VZ), a proliferative ventricular epithelium positioned below the developing cortex. The $\mathrm{VZ}$ is populated by radial glia, the neuronal progenitor cells of the CNS (Malatesta et al., 2000, 2003; Miyata et al., 2001; Noctor et al., 2001; Anthony et al., 2004). As development of the cerebral cortex progresses, cell cycle time in radial glia increases, as does the number of neurogenic divisions (Miyama et al., 1997). Slowing of the cell cycle within the VZ has been proposed to lead to neurogenic, rather than proliferative, cell divisions (Calegari \& Huttner, 2003).

Sauer (1935) first described the process of interkinetic nuclear migration (INM), whereby the nuclei of dividing radial glia migrate between the basal VZ, where they reside during the S phase, to the apical surface of the VZ where mitosis occurs. Neurogenesis also occurs in the subventricular zone (SVZ), where basal neuronal progenitors undergo mitosis (Haubensak et al., 2004; Miyata et al., 2004; Caligari et al., 2005). The identification of mu opioid receptors (MOR) on proliferating radial glia in the mouse (Sargeant et al., 2007) and on human neural progenitors of the SVZ (Tripathi et al., 2008) suggests activation of the receptor by opioids could potentially modulate the behaviour of cortical progenitor cells.

Morphine is the prototypic ligand for the MOR and is an opiate alkaloid commonly used for analgesia, as well as being labelled a drug

Correspondence: Dr D. J. Day, as above.

E-mail: darrenday@vuwacnz

Received 9 December 2007, revised 10 July 2008, accepted 19 July 2008 of abuse. Morphine perturbs aspects of brain development in rodents, including having effects on DNA synthesis (Miller et al., 1982; Kornblum et al., 1987; Hauser et al., 2000; Stiene-Martin et al., 2001) as well as altering numbers of cortical neurons (Seatriz \& Hammer, 1993). More recently, mu and kappa opioid receptors have been shown to modulate neurogenesis in embryonic stem cells and human neuronal progenitors (Kim et al.,2006; Sheng et al, 2007). Opioids also modulate neurogenesis in adult systems. Knockout (KO) studies have shown beta-endorphin is required for exercise-stimulated increases in hippocampal neurogenesis in the adult mouse (Koehl et al., 2008), and the MOR represses hippocampal neurogenesis in response to ischaemia (Kolodziej et al., 2008). Given the known actions of opiates on cellular proliferation, we speculated that morphine exposure during the period of corticogenesis may alter cell cycle progression in dividing radial glia, potentially modulating neuronal output, given the putative relationship between cell cycle duration and outcome of radial glial divisions (Takahashi et al, 1999). Using a time course of bromodeoxyuridine (BrdU) pulse labelling and immunohistochemical (IHC) detection of mitotic nuclei, we report the effect of acute morphine treatment on cell cycle progression in the developing cortex of the E15.5 mouse.

\section{Materials and methods}

Animals

All animal experimentation was approved by the Victoria University of Wellington animal ethics committee. C57BL/6J and MOR KO mice backcrossed onto a C57BL/6J background (MOR KO) were mated overnight (21:00-09:00 hours). Mice that had vaginal plugs present in the morning were defined as E0. The MOR KO strain was

(c) The Authors (2008). Journal Compilation (c) Federation of European Neuroscience Societies and Blackwell Publishing Ltd 


\section{T. J. Sargeant et al.}

kindly provided by Dr K. J. Christie, and its origins have been previously described in detail (Schuller et al., 1999; Vaughan et al., 2003).

\section{Morphine and BrdU treatment}

On the morning of E15 (06:00 hours), pregnant dams were injected subcutaneously with either $10 \mathrm{mg} / \mathrm{kg}$ morphine sulphate or a sham treatment of an equivalent volume of saline. The same dose of morphine or saline was administered every $3 \mathrm{~h}$. Six hours after the first injection of morphine, animals were given $\mathrm{BrdU}(50 \mathrm{mg} / \mathrm{kg})$ intraperitoneally. Animals were killed by cervical dislocation at selected time-points.

\section{Measurement of proliferation and INM}

To determine whether morphine treatment altered the number of proliferative cells in the $\mathrm{S}$ phase, three morphine-treated and three saline-treated controls were given BrdU, then killed $1 \mathrm{~h}$ later and the pups processed for IHC staining for BrdU incorporation. BrdUpositive nuclei in the dorsal-lateral VZ at the coronal level of the first anterior appearance of the third ventricle were counted in a $200-\mu \mathrm{m}-$ wide column, perpendicular to the ventricular surface. Sections matched to this brain region were used throughout the study.

To determine whether morphine altered progression of INM, pregnant dams were given BrdU, then killed at hourly intervals over a 6-h period. Pups from nine morphine-treated and eight saline-treated controls were examined, along with pups from five morphine-treated MOR KO and six saline-treated MOR KO controls. To assess INM, the same column of VZ used for determining the number of proliferative cells was stratified into $8 \times 20$ - $\mu \mathrm{m}$ bins from the apical
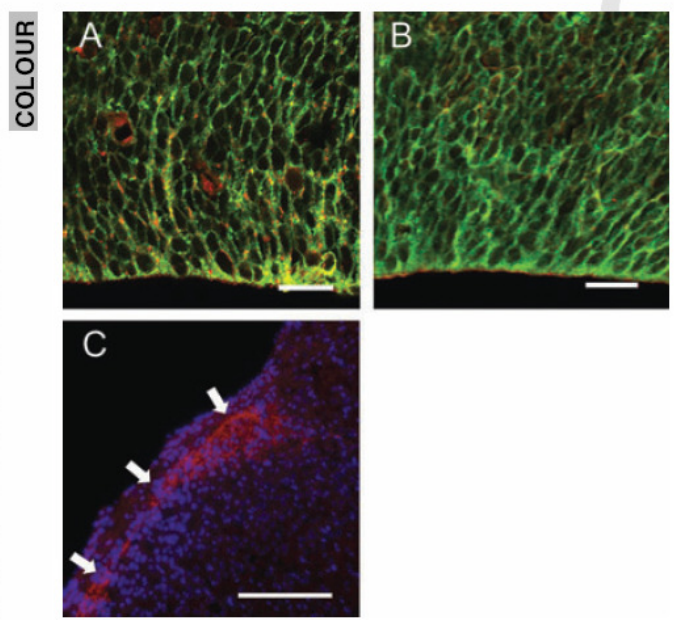

FIG. 1. MOR and DOR immunoreactivity on GLAST-positive radial glia of the E15.5 VZ. MOR immunoreactivity (red) is found on GLAST-positive radial glia (green) of the VZ (A). Using an anti-DOR antibody, minimal DOR immunoreactivity (red, B) was seen on radial glia (green), whereas staining of adult mouse spinal trigeminal tract showed strong staining (red) with the same antibody and conditions (C, white arrows). Cell nuclei in $(\mathrm{C})$ are counterstained with DAPI (blue). Scale bars: $20 \mu \mathrm{m}$ (A and B); $200 \mu \mathrm{m}$ (C). surface of the VZ. BrdU-positive nuclei were considered to reside within a particular bin if they lay within or on the lower-most boundary of that bin.

\section{Measuring differences in $G_{2} / M$ transition}

To determine the effect of morphine on $\mathrm{G}_{2} / \mathrm{M}$ phase progression, morphine-treated dams and saline-treated control animals were killed $1.5,2$ and $2.5 \mathrm{~h}$ after BrdU injection. Staining for both BrdU and mitotic profiles with phospho-histone $\mathrm{H} 3$ was undertaken, and the fraction of phospho-histone $\mathrm{H} 3$-stained nuclei and BrdU/phosphohistone $\mathrm{H} 3$ dual-labelled nuclei determined for the same area of ventricle as described for the 1-h BrdU-labelled animals. At least 200 phospho-histone $\mathrm{H} 3$-stained nuclei per animal were counted.

\section{Morphine treatment and exit from the proliferative pool}

To determine whether morphine treatment altered the number of cells that re-entered the cell cycle, pregnant dams (eight morphine-treated

$1 \mathrm{~h}$

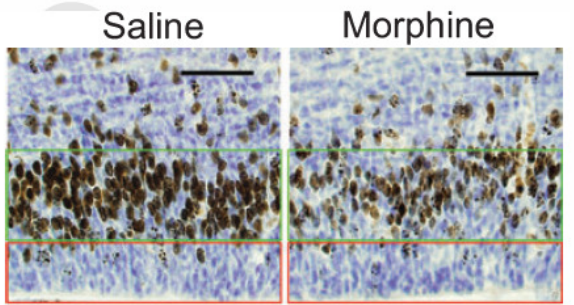

$3 \mathrm{~h}$

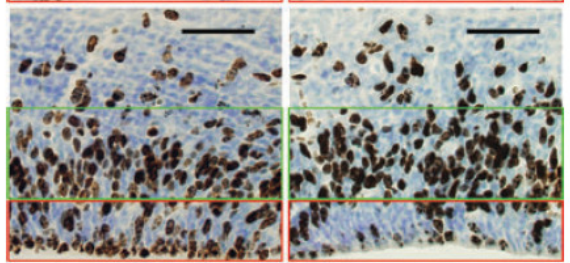

$5 \mathrm{~h}$

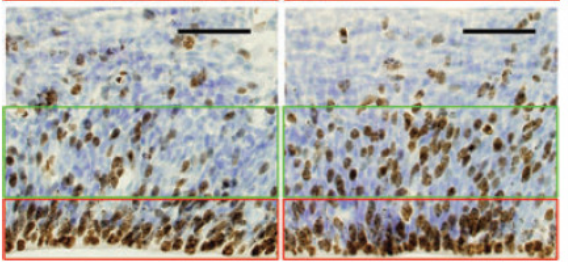

FIG. 2. Acute morphine treatment slows INM in the VZ of the lateral ventricle in the E15.5 mouse. Regions used for cell counting in the basal VZ and apical $\mathrm{VZ}$ are indicated by green and red boxes, respectively. Nuclei were counted as being in the apical VZ if they resided within $40 \mu \mathrm{m}$ of the lateral ventricle wall, nuclei if they were in a $60-\mu \mathrm{m}$ region above the apical VZ. One hour after BrdU administration, BrdU staining (brown) is seen in the basal VZ where cells are undergoing $\mathrm{S}$ phase. The saline-treated control animals and morphinetreated animals look similar; however, by $3 \mathrm{~h}$ after BrdU labelling nuclei have migrated to the apical VZ during the $\mathrm{G}_{2}$ phase and it can be seen that fewer cells have accumulated at the apical surface of the $\mathrm{VZ}$ in the morphine-treated animals. By 5 h post-labelling it can be seen that more BrdU-labelled nuclei are left in the basal VZ in the morphine-treated animals. Scale bars: $50 \mu \mathrm{m}$. 
and five saline-treated controls) were administered BrdU, then killed $16 \mathrm{~h}$ later and stained for $\mathrm{BrdU}$ and Ki67. Animals received $10 \mathrm{mg} / \mathrm{kg}$ doses of morphine (or an equivalent volume of saline)

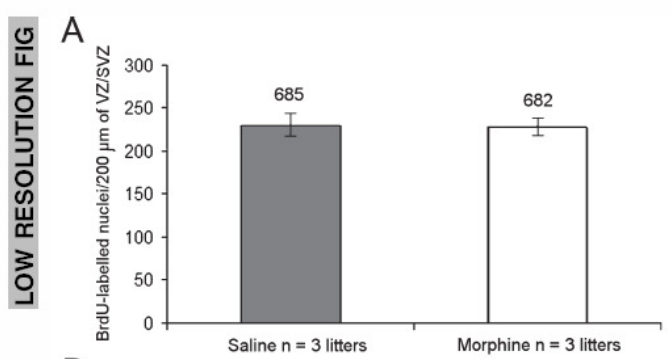

B

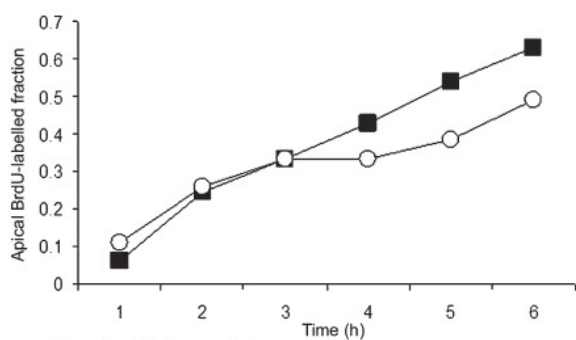

Litters used/number of cells countec Saline

$\begin{array}{lcccccc}\mathrm{n} \text { (litters) } & 1 & 1 & 1 & 2 & 2 & 1 \\ \text { Cells counted } & 269 & 251 & 350 & 527 & 696 & 355\end{array}$

Cells count

$\begin{array}{lcccccc}\mathrm{n} \text { (litters) } & 1 & 1 & 1 & 2 & 2 & 2 \\ \text { Colls counted } & 233 & 299 & 276 & 601 & 670 & 687\end{array}$

\section{C}

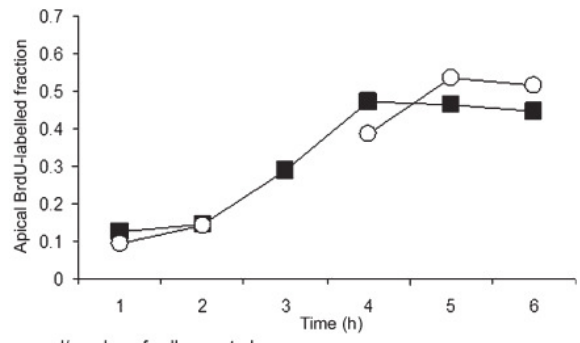

Litters used/number of cells counted

Saline

n (itters)

$\begin{array}{lcccccc}n \text { (inters) } & 1 & 1 & 1 & 1 & 1 & 1 \\ \text { Cells counted } & 241 & 241 & 243 & 307 & 347 & 458\end{array}$

Morphine

$\begin{array}{lcccccc}\mathrm{n} \text { (litters) } & 1 & 1 & 0 & 1 & 1 & 1\end{array}$

$\begin{array}{lllllll}\text { Cells counted } & 210 & 216 & \text { NA } & 321 & 324 & 346\end{array}$ subcutaneously every $3 \mathrm{~h}$ until $6 \mathrm{~h}$ after the BrdU injection, after which morphine was given every $6 \mathrm{~h}$ until death. Animals were E15.5 at the time of death, with the first morphine injection being given at E14.5.

Exit from the proliferative pool (cell cycle exit) was determined by staining for $\mathrm{BrdU}$ and $\mathrm{Ki} 67$, counting BrdU-labelled cells, then determining the fraction that also stained for Ki67. BrdU/Ki67 duallabelling has been used previously to assess cell cycle re-entry (Chenn \& Walsh, 2002; Hodge et al., 2004). Ki67 is a marker that is restricted to cells that are in the $\mathrm{G}_{1}, \mathrm{~S}, \mathrm{G}_{2}$ or $\mathrm{M}$ phase of the cell cycle. Ki67 is not detectable in cells in the $\mathrm{G}_{0}$ phase under normal conditions (Gerdes et al., 1984; Endl et al., 1997), but has been found in the $\mathrm{G}_{0}$ 3 phase cell nucleoli in very minimal amounts (Bullwinkel et al., 2006). Stained nuclei were counted in a $200-\mu \mathrm{m}$-wide column comprising the most medial and most lateral regions of the dorsal VZ/SVZ Approximately $600 \mathrm{BrdU}$-positive nuclei were counted for each of the medial and lateral regions. The slides were coded such that the researcher counting was unaware whether they originated from a morphine-treated or saline-treated animal.

\section{Tissue preparation}

Pregnant dams were killed by cervical dislocation, and the embryos rapidly removed and whole heads fixed overnight at $4{ }^{\circ} \mathrm{C}$ in phosphate-buffered saline (PBS) containing 4\% paraformaldehyde. Embryo heads were either dehydrated through a graded ethanol/xylene series and embedded in paraffin wax, or they were cryoprotected in PBS containing 30\% sucrose. Paraffin-embedded tissue was cut into $10-\mu \mathrm{m}$-thick sections on an 820 series microtome (American Optical, Buffalo, NY, USA), while cryopreserved frozen tissue was sectioned at $20 \mu \mathrm{m}$ on an HM 500 OM series cryostat microtome (Microm, Heidelberg, Germany). All sections were mounted on Superfrost plus slides (Esco, Erie Scientific, Portsmouth, $\mathrm{NH}$, USA)

IHC

IHC, including antigen retrieval steps, was performed as previously reported (Sargeant et al., 2007). Antibodies used were guinea pig antiGLAST (1: 1000, AB 1782, Chemicon), rabbit anti-MOR (1:1000, AB5511, Chemicon), rabbit anti-delta opioid receptor (DOR; 1: 1000, AB5503, Chemicon), rabbit anti-phospho-histone $\mathrm{H} 3$ (1: 1000, Abcam, AB5176), rabbit anti-Ki67 (1:25, Ab15580, Abcam), anti-BrdU-biotin conjugate ( $1: 25$, Invitrogen, MD5215), mouse anti-BrdU-Alexa 546 conjugate (1: 100, A21308, Invitrogen), mouse anti-BrdU-Alexa 488 conjugate ( $1: 100$, A21303, Invitrogen), goat anti-rabbit-Alexa 555 conjugate (1: 1000, Invitrogen, A-21428), streptavidin-Alexa 488 (1:1000, Invitrogen, S-32354) and goat

FIG. 3. Morphine effects on proliferation and INM in the E15.5 mouse dorsa telencephalon. (A-D) The relative fraction of bromodeoxyuridine (BrdU)labelled nuclei in the basal and apical ventricular zone (VZ) in the experimen described in Fig. 2. (A) Morphine treatment did not change the sum total of BrdU-labelled nuclei counted in the basal and apical VZ $1 \mathrm{~h}$ after BrdU labelling compared with saline-treated control animals (values are the mean \pm SEM). (B) The fraction of BrdU-positive nuclei counted within the pical VZ, expressed as a fraction of the total number of BrdU-labelled nucle counted. The number of litters analysed and labelled nuclei counted for each ime-point are as indicated. (B) Morphine-treated animals (?) exhibited lowe proportions of total BrdU-labelled nuclei in the apical VZ compared with saline-treated animals $(\triangle)$. (C) In the MOR KO mouse, morphine treatment did not slow the accumulation of BrdU-positive nuclei into the apical VZ. SVZ, subventricular zone.

() The Authors (2008). Journal Compilation (ㄷ) Federation of European Neuroscience Societies and Blackwell Publishing Ltd

European Journal of Neuroscience, $1-8$ 


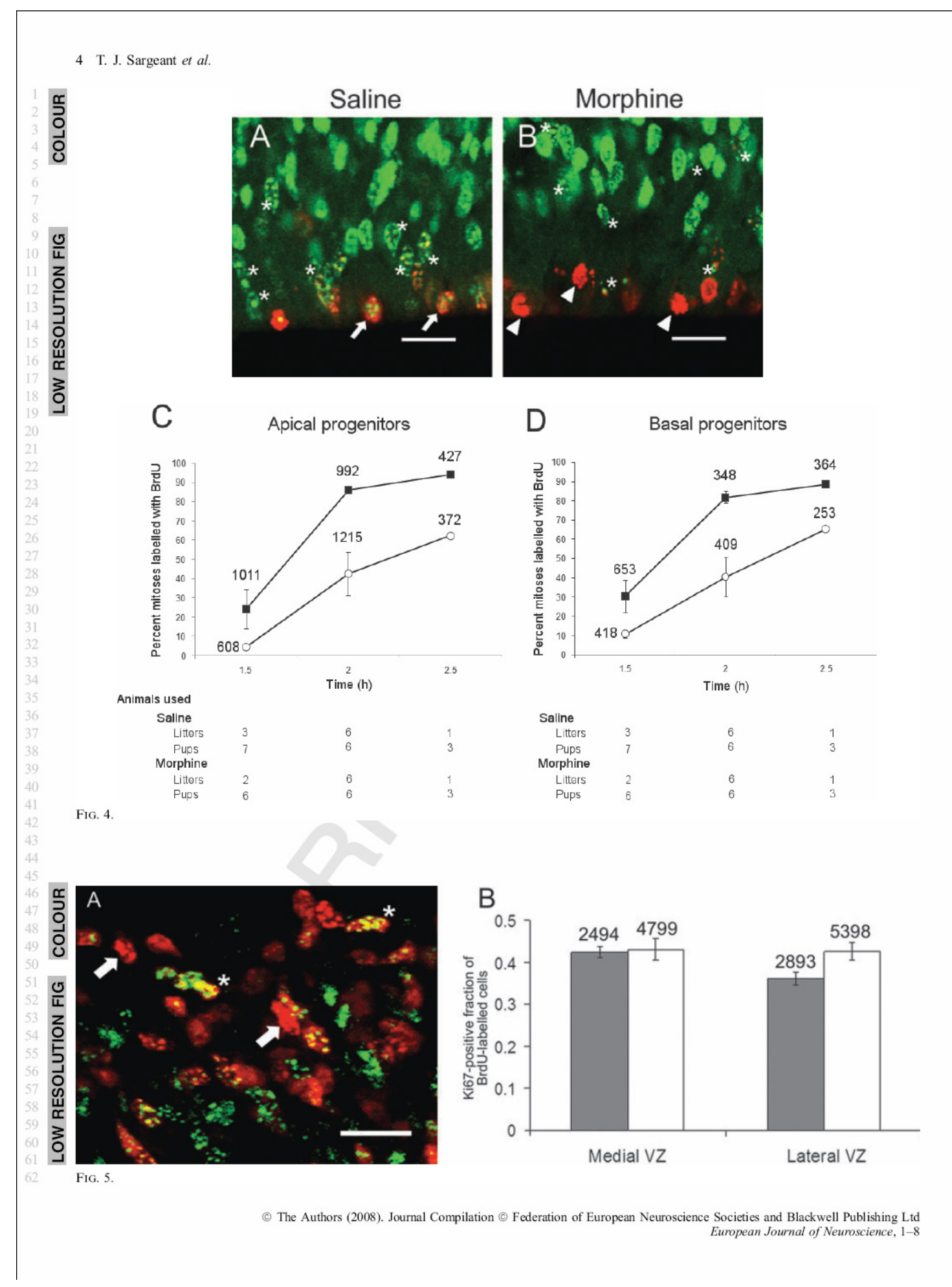


anti-guinea pig IgG-Alexa 488 conjugate $(1: 1000$, A11073, Invitrogen). Post-fixation for $5 \mathrm{~min}$ in PBS containing $4 \%$ paraformaldehyde was required when using the anti-BrdU-Alexa 546 conjugate to prevent diffusive spread of the immunoreactivity signal in the sections.

\section{Imaging and cell counting}

Brightfield images were captured using an Olympus AX70 photomicroscope (Olympus Optical, Hamburg, Germany) equipped with an Olympus DP70 CCD camera. Confocal microscopy was performed using an Olympus FluoView FV1000 microscope. Confocal images were overlaid and labelled using OLYMPUS fluOvIEw vl.6a Viewer and edited with ADOBE PHOTOSHOP CS3 software for presentation purposes. Cells were counted using IMAGEJ software (v. 1.37, NIH, USA).

\section{Statistical analysis}

Statistical analysis of data was performed using spss for Windows v14.0 (SPSS, Chicago, IL, USA). Data taken from hourly INM measurements of migrating BrdU cohorts were analysed by an ANCOVA test to correct for time as a covariate. In the present study all $n$ values were strictly defined as being one pregnant dam (Haseman \& Hogan, 1975; Holson \& Pearce, 1992; Zorrilla, 1997).

\section{Results}

\section{MOR and DOR staining}

As we have previously reported (Sargeant et al, 2007), MOR immunoreactivity (red) is detected on GLAST-positive cells (green) of the dorsal VZ (Fig. 1A). In contrast, minimal DOR immunoreactivity (red, Fig. 1B) was detected on the same GLAST-positive cells. Staining of the adult spinal trigeminal tract as a positive control for the DOR antibody gave the expected pattern of intense staining using the same staining parameters as those described for Fig. 1B (Fig. 1C).

\section{Morphine slows INM}

Representative images of BrdU-labelled nuclei migrating from the basal VZ to the apical VZ are shown as a time course for morphine- treated animals and saline-treated controls (Fig. 2). One hour after BrdU administration, nuclei from cells in the $\mathrm{S}$ phase reside in the basal VZ, but by $3 \mathrm{~h}$ post-BrdU treatment, a cohort of labelled nucle have undergone INM and have moved to the apical VZ where they undergo mitotic division. Morphine treatment delays INM such that by $3 \mathrm{~h}$ post-BrdU treatment few labelled nuclei are present at the lateral ventricular wall in morphine-treated animals, whereas, in salinetreated controls, a large number of BrdU-labelled nuclei are seen at the ventricular wall. Quantitative analysis shows that morphine treatment did not change the number of cells labelled by BrdU (Fig. 3A), but delayed (or slowed) INM such that it took longer for labelled nuclei to reach the apical VZ $(P=0.013$, ANCOVA $)$ and longer to leave the basal VZ $(P=0.036$, ANCOVA; Fig. 3B and C). Slower INM afte morphine treatment did not occur when the experiment was repeated using a MOR KO mouse strain (Fig. 3D). No pyknotic nuclei were observed in any of the experimental groups.

\section{Relative $G_{2} / M$ phase measurement}

To corroborate the results presented in Figs 2 and 3, we examined the effect morphine treatment had upon the time required for $\mathrm{G}_{2} / \mathrm{M}$ transition by labelling $\mathrm{S}$ phase cells with a single pulse of $\mathrm{BrdU}$ and then, $1.5,2$ and $2.5 \mathrm{~h}$ later, killing the animals and determining the number of mitotic profiles that showed BrdU labelling. Cells displaying mitotic profiles were detected by intense staining for phospho-histone H3. Figure 4 shows representative photomicrograph of BrdU-labelled nuclei (green) and phospho-histone H3-stained nuclei (red) $2 \mathrm{~h}$ after BrdU administration. Figure 4A shows staining from a saline-treated control, and Fig. 4B shows staining from a morphine-treated animal. Cells that are in late $\mathrm{S}$ phase have punctate BrdU staining (asterisks) and, as expected, reach the apical VZ ahead of those nuclei that show solid BrdU staining. Figure 4A shows that in the saline-treated animals, punctate $\mathrm{BrdU} /$ phospho-histone $\mathrm{H} 3$ staining can be seen at the lateral ventricle wall in some cells (white arrows), and that more late $\mathrm{S}$ phase-labelled nuclei have reached the apical VZ. In the morphine-treated animals, fewer mitotic profiles are BrdU labelled, and fewer late $\mathrm{S}$ phase-labelled nuclei have migrated to the apical VZ by INM.

Figure 4C and D shows the fraction of phospho-histone H3-labelled nuclei that also stained for BrdU in the apical and basal VZ for salinetreated controls and morphine-treated dams. For the saline-treated control at $2 \mathrm{~h}$ after $\mathrm{BrdU}$ injection, $86 \%$ of the apical phospho-histone

11FIG. 4. Morphine treatment slows $\mathrm{G}_{2}$ progression in the E15.5 pup. (A and B) Staining of nuclei for phospho-histone $\mathrm{H} 3$ (red) and bromodeoxyuridine (BrdU; green) in a representative saline-treated control pup (A) and a morphine-treated pup (B) $2 \mathrm{~h}$ after BrdU injection. Many of the phospho-histone H3-labelled mitotic

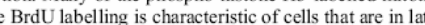
$\mathrm{S}$ phase at the onset of BrdU labelling (asterisks). In the morphine-treated animals, fewer BrdU-positive nuclei displaying punctate labelling are located near the apical surface of the VZ (B, asterisks). Similarly, fewer of the apical mitoses in morphine-treated animals stain for BrdU and thus appear red only (arrowhead). Scale bars: $20 \mu \mathrm{m}$. (C) The fraction of phospho-histone H3-stained nuclei (mitotic) in the apical VZ that also stain for BrdU. Over the time course assayed (1.5, 2.0 and $2.5 \mathrm{~h}$ post-BrdU injection) saline-treated controls $(\searrow$ ) display more rapid BrdU labelling of mitoses than morphine-treated animals (?). The delay in BrdU labellin of mitoses seen in morphine-treated animals is approximately half an hour. (D) Basal progenitor cells display a similar response to morphine exposure. The total numbers of pups and litters used for each time-point appear under their corresponding time-points and the total number of mitosis counted adjacent to each timepoint. The fraction of dual-labelled mitoses is expressed as the mean \pm SEM. All means represent the means of litters, not littermate replicates. Standard error was not calculated for the $2.5 \mathrm{~h}$ time-points as only two-three pups from a single litter in each group were used for analysis. FIG. 5. Morphine does not affect cell cycle exit. Staining for the proliferation marker Ki67 (green) and bromodeoxyuridine (BrdU; red) was performed $16 \mathrm{~h}$ after
BrdU administration. Confocal imaging (A) was used to detect labelled nuclei (red) that have exited the cell cycle and therefore do not show Ki67 immunoreactivity BrdU administration. Confocal imaging (A) was used to detect labelled nuclei (red) that have exited the cell cycle and therefore do not show Ki67 immunoreactivity
(green, white arrows). BrdU-positive nuclei that are still proliferative also stain for Ki67 and thus appear yellow/orange (white asterisks). (B) Morphine treatment (green, white arrows). BrdU-positive nuclei that are still proliferative also stain for Ki67 and thus appear yellow/orange (white asterisks). (B) Morphine treatment
(white bars) did not alter the fraction of dual-labelled Ki67/BrdU-postive nuclei compared with saline-treated control animals (grey bars) in either the medial or the (white bars) did not alter the fraction of dual-labelled Ki67/ BrdU-postive nuclei compared with saline-treated control animals (grey bars) in either the medial or the morphine-treated dams). The values above each bar represent the number of BrdU-stained nuclei counted. Scale bar: 20 um.

() The Authors (2008). Journal Compilation () Federation of European Neuroscience Societies and Blackwell Publishing Ltd European Journal of Neuroscience, 1 - 


\section{T. J. Sargeant et al.}

$\mathrm{H} 3$-stained mitotic profiles also stained for BrdU, while in the morphine-treated cohort only $43 \%$ were dual-labelled. Similarly, $82 \%$ of the phospho-histone H3-positive basal progenitors also stained for $\mathrm{BrdU}$ in the saline-treated group, while only $41 \%$ were dual-labelled in the morphine-treated group. Figure $4 \mathrm{C}$ and $\mathrm{D}$ also shows that the morphine-induced delay in BrdU labelling of mitotic nuclei at both apical and basal positions was approximately $0.5 \mathrm{~h}$.

\section{Measurement of cell cycle exit}

To determine whether acute morphine exposure influences whether progenitor cells undergo a proliferative division to yield another radia glial cell and a quiescent neuron, or exit the cell cycle entirely by dividing to form two quiescent cells, the same cohort of animals used to analyse the effect of morphine on cell cycle progression were stained for BrdU and the proliferation marker Ki67. Ki67 is expressed on replicative cells but not on post-mitotic neurons that have entered $\mathrm{G}_{0}$. Thus, $16 \mathrm{~h}$ after BrdU treatment, nuclei that have stained positively for both BrdU and Ki67 have remained within the cel cycle, whereas nuclei that have stained for BrdU alone have exited the cell cycle. The fraction of BrdU-positive nuclei that stained for Ki67 and the fraction of nuclei that stained for BrdU alone were determine in coronal sections of lateral ventricle divided into medial and latera portions ( $n=5$ saline-treated controls, eight morphine-treated animals). Figure $5 \mathrm{~A}$ shows a representative region of basal VZ that contains nuclei that have stained for BrdU alone (red, white arrows), while cells that co-stained for $\mathrm{Ki} 67$ (green) and BrdU appea yellow/orange (asterisks). Morphine treatment did not significantly alter the number of BrdU/Ki67-positive cells in either the medial o lateral portions of the VZ $(P=0.093$; Fig. 5)

\section{Discussion}

In this paper, we have shown that activation of opioid receptors by morphine influences cell cycle progression by slowing the $G_{2} / M$ phase transition. We have recently reported that radial glia in the foeta mouse brain express the MOR (Sargeant et al., 2007), and we now provide evidence in the present study for the biological consequences of opioid receptor activation by morphine. Although morphine acts predominantly through the MOR, it can also activate delta and kappa opioid receptors depending upon the dose administered. The dose we used is similar to that used in other mouse studies (Stiene-Martin et al., 2001), although it is not possible to determine the transplacental dose that the developing embryonic brain is exposed to. The effects we observed are, however, likely to be mediated via MOR as slowing of INM was not detected when the experiment was undertaken with a MOR KO strain. Additionally, only minimal levels of DOR were detected on radial glia (Fig. 1B). It is still possible that morphine may be acting indirectly on radial glia and basal progenitor cells at a paracrine or endocrine level, for example by modulating the hypothalamic-pituitary-adrenal axis to influence hormone levels (Spiegel et al., 1982). The expression of MOR on radial glia, in our opinion, favours a mechanism for the action of morphine on radial glia that express the MOR, but whether morphine is acting directly on MOR on radial glia or not remains to be unequivocally determined.

The data presented in Figs 2-4 support the view that in vivo morphine exposure in the developing rodent brain lengthens the $\mathrm{G}_{2} / \mathrm{M}$ phase. The increased duration for morphine-exposed cells to transition $\mathrm{G}_{2} / \mathrm{M}$ phase and undergo mitosis could be accounted for either by a stall at the beginning of $\mathrm{G}_{2}\left(\mathrm{G}_{2}\right.$-checkpoint) or by a decreased rate of progression. The existence of a transient, early $\mathrm{G}_{2}$-checkpoint has been reported (Xu et al., 2002), but it is not possible with the experiments that we have undertaken to distinguish between delay at an early $\mathrm{G}_{2}$-checkpoint or slower INM.

In contrast to the present study, Reznikov et al. (1999) reported that treatment with DAMGO and bremazocine (which act on mu and kappa opioid receptors, respectively) increased $\left[{ }^{3} \mathrm{H}\right]$ thymidine labelling within the $\mathrm{VZ}$ due to lengthening of the $\mathrm{S}$ phase in replicating cells. We did not detect a change in the number of proliferative cells in either basal or apical progenitor populations. We feel that the study by Reznikov et al. (1999) must be treated with caution, as multiple pups were analysed from a single pregnant female for each experimental group. Teratological studies that use multiparous species, such as mice, are confounded by litter effects if the study selects littermates (within a litter) as its base definition of sample size (Haseman \& Hogan, 1975; Holson \& Pearce, 1992; Zorrilla, 1997).

During normal rodent cortical development there is an increase in cell cycle duration as development progresses primarily attributed to an increase in the duration of the $\mathrm{G}_{1}$ phase with $\mathrm{G}_{2} / \mathrm{M}$ not changing significantly (Takahashi et al., 1999); however, during retinal development there is a significant lengthening of $\mathrm{G}_{2} / \mathrm{M}$ (Alexiades \& Cepko, 1996). Interestingly, a lengthening of $\mathrm{G}_{2} / \mathrm{M}$ is seen in cortical precursors from mice lacking beta-amyloid precursor protein (López-Sánchez et al., 2005). Together these studies support a functional role for regulation of $\mathrm{G}_{2} / \mathrm{M}$ duration in cortical development and pathogenesis.

Modulation of the duration of the $\mathrm{G}_{2}$ phase by opiates has been reported in other brain regions and other cellular systems. For example, chronic morphine exposure in the adult rodent hippocampus in vivo markedly decreased cellular proliferation (Eisch et al., 2000) and shortened the $\mathrm{G}_{2}$ phase (Mandyam et al., 2004). In vitro experiments using the non-selective opiate ligands ethylketocyclazocine and etorphine, which have low nanomolar affinity for all three classical opioid receptors, blocked T47D breast cancer cells in the $\mathrm{G}_{2} / \mathrm{M}$ phase (Panagiotou et al., 1999). This study showed that an opiate-induced cell cycle block occurred simultaneously with an opiate-induced modification of the actin/tubulin cytoskeleton. These data are consistent with our observation that morphine alters INM, a process that is sensitive to microfilament disruption by cytochalasin B (Messier \& Auclair, 1974; Murciano et al., 2002). Interestingly, it has also been shown that when astrocytes were cultured from neonatal mice, a disproportionately large number of MOR-positive astrocytes were in $\mathrm{G}_{2} / \mathrm{M}$ (Stiene-Martin et al., 1998).

Our observation that both basal and apical mitoses showed morphine-induced lengthening of the $\mathrm{G}_{2} / \mathrm{M}$ phase suggests that morphine acts by activating an early $\mathrm{G}_{2}$-checkpoint in the cell cycle rather than by inhibiting INM as basal progenitors do not undergo INM. Slowing of INM probably occurs as INM and $G_{2}$ phase are intrinsically linked processes in radial glia. Interestingly, the studies of Cappello et al. (2006) and Tamai et al. (2007) showed that INM in the $\mathrm{CDC} 42 \mathrm{KO}$ mouse and in the Pax6 $\mathrm{KO}$ rat resulted in an uncoupling of $\mathrm{G}_{2}$ phase duration and INM. In both these studies, disruption of INM caused ectopic mitoses. Ueno et al. (2006) have shown that cell cycle progression is required for normal INM to the apical surface of the VZ. That morphine allows the coordination of cell cycle progression and INM to remain intact while other studies have demonstrated that they can be easily uncoupled (Messier \& Auclair, 1974; Murciano et al., 2002) is most likely a consequence of morphine being a milder cellular challenge than the reagents used in the above studies.

The VZ of the embryonic brain contains gradients of important extrinsic fate determinants, such as the anti-neurogenic Notchl (as well as the Notch1 ligand, Delta 1; Murciano et al., 2002). Expression 
of Notch 1 is restricted to the ventricular wall, and BrdU labelling has shown that Notch 1 is expressed by cells in the $\mathrm{G}_{2}$ phase, but not in cells in the $\mathrm{S}$ phase or early $\mathrm{G}_{2}$ (Murciano et al., 2002). Regulation of the duration of the $\mathrm{G}_{2}$ phase by opioids could potentially be a mechanism that controls exposure of cells to ligands that determine cell fate. Although in our study morphine was not observed to alter cell cycle exit, it cannot be ruled out that morphine may influence developmental maturation and cell fate, as has been shown in cultures of embryonic stem cells (Kim et al., 2006).

In the present study, we have shown that morphine exposure in utero mediates acute effects on progenitor cell behaviour within the $\mathrm{VZ}$ of the developing telencephalon. Acute exposure to morphine slows the arrival of radial glial nuclei at the apical surface of the lateral ventricle, as well as increases the duration of the $\mathrm{G}_{2}$ phase of the cell cycle. These effects induce a slowing of the overall cell cycle, although it is likely that some phases of the cell cycle are left undisturbed. The biological implications and the developmental impact of opioidergic perturbation of the cortical progenitor cell cycle in vivo merit further investigation.

\section{Acknowledgements}

This work was supported by grants from the Wellington Medical Research Foundation, Inc. T.J.S. was supported by a Bright Future PhD scholarship (Tertiary Education Commission, NZ). We would like to acknowledge Stefan Teufel and Tom Fanning for help with sectioning.

\section{Abbreviations}

BrdU, bromodeoxyuridine; DOR, delta opioid receptor; E, embryonic day; IHC, immunohistochemistry; INM, interkinetic nuclear migration; KO, knockout; MOR, mu opioid receptor; PBS, phosphate-buffered saline; SVZ, subventricular zone; VZ, ventricular zone.

\section{References}

Alexiades, M.R. \& Cepko, C. (1996) Quantitative analysis of proliferation and cell cycle length during development of the rat retina. Dev. Dyn., 205, 293-307. Anthony, T.E., Klein, C., Fishell, G. \& Heintz, N. (2004) Radial glia serve as neuronal progenitors in all regions of the central nervous system. Neuron, $\mathbf{4 1}$ $881-890$

Calegari, F. \& Huttner, W.B. (2003) An inhibition of cyclin-dependent kinases that lengthens, but does not arrest, neuroepithelial cell cycle induces premature neurogenesis. J. Cell Sci., 116, 4947-4955.

Caligari, F., Haubensak, W., Haffner, C. \& Huttner, W.B. (2005) Selective lengthening of the cell cycle in the neurogenic subpopulation of neural progenitor cells during mouse brain development. $J$. Neurosci., 25, 65336538

Cappello, S., Attardo, A., Wu, X., Iwasato, T., Itohara, S., Wilsch-Brauninger, M., Eilken, H.M., Rieger, M.A., Schroeder, T.T., Huttner, W.B., Brakebusch, C. \& Gotz, M. (2006) The Rho-GTPase cdc42 regulates neural progenitor fate at the apical surface. Nat. Neurosci., 9, 1099-1107.

Caviness, V.S. Jr, Goto, T., Tarui, T., Takahashi, T., Bhide, P.G. \& Nowakowski, R.S. (2003) Cell output, cell cycle duration and neuronal specification: a model of integrated mechanisms of the neocortical proliferative process. Cereb. Cortex, 13, 592-598.

Chenn, A. \& Walsh, C.A. (2002) Regulation of cerebral cortical size by control of cell cycle exit in neural precursors. Science, 297, 365-369.

Eisch, A.J., Barrot, M., Schad, C.A., Self, D.W. \& Nestler, E.J. (2000) Opiates inhibit neurogenesis in the adult rat hippocampus. Proc. Natl. Acad. Sci. U.S.A., $97,7579-7584$

Endl, E., Steinbach, P., Knüchel, R. \& Hofstädter, F. (1997) Analysis of cell cycle-related $\mathrm{Ki}-67$ and $\mathrm{pl} 20$ expression by flow cytometric BrdUrdHoechst/7AAD and immunolabeling technique. Cytometry, 29, 233-241. Gerdes, J., Lemke, H., Baisch, H., Wacker, H.H., Schwab, U. \& Stein, H (1984) Cell cycle analysis of a cell proliferation-associated human nuclear
Haseman, J.K. \& Hogan, M.D. (1975) Selection of the experimental unit in teratology studies. Teratology, 12, 165-171.

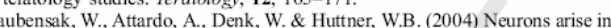
the basal neuroepithelium of the early mammalian telencephalon: a major site of neurogenesis. Proc. Natl. Acad. Sci. U.S.A., 101, 3196-3201.

Hauser, K. F. Houdi, A A. Tuhek, C. S., Elde, R. P. \& Maxson, W. (2000) Opioids intrinsically inhibit the genesis of mouse cerebellar grnule (2000) precursors in vitro: differential impact of mu and delta receptor activation proliferation and neurite elongation. Eur. J. Neurosci., 12, 1281-1293. Hendzel, M J. Wei, Y Mancini, M. A. Van Hooser, A, Ranalli, T. Brinkley, endzel, M.J., Wei, Y., Mancini, M.A., Van Hooser, A., Ranalli, T., Brinkley, B.R., Bazett-Jones, D.P. \& Allis, C.D. (1997) Mitosis-specific phosphory-
lation of histone H3 initiates primarily within pericentromeric heterochromatin during G2 and spreads in an ordered fashion coincident with mitotic chromosome condensation. Chromosoma, 106, 348-360.

Hodge, R.D., D'Ercole, A.J. \& O'Kusky, J.R. (2004) Insulin-like growth factor-I accelerates the cell cycle by decreasing G1 phase length and increases cell cycle reentry in the embryonic cerebral cortex. $J$. Neurosci. 24, 10201-10210.

Holson, R.R. \& Pearce, B. (1992) Principles and pitfalls in the analysis of prenatal treatment effects in multiparous species. Neurotoxicol. Teratol., 14, $221-228$.

Kim, E., Clark, A.L., Kiss, A., Hahn, J.W., Wesselschmidt, R., Coscia, C.J. \& Belcheva, M.M. (2006) $\mu$ - and $\kappa$-opioids induce the differentiation of embryonic stem cells to neural progenitors. J. Biol. Chem., 281, 3374933760

Koehl, M., Meerlo, P., Gonzales, D., Rontal, A., Turek, F.W. \& Abrous, D.N. (2008) Exercise-induced promotion of hippocampal cell proliferation requires \{beta\}-endorphin. FASEB J., Epub ahead of print ???, ???

Kolodziej, A., Stumm, R., Becker, A. \& Höllt, V. (2008) Endogenous opioids inhibit ischemia-induced generation of immature hippocampal neurons via the mu-opioid receptor. Eur. J. Neurosci, 27, 1311-1319.

Kornblum, H.I., Loughlin, S.E. \& Leslie, F.M. (1987) Effects of morphine on DNA synthesis in neonatal rat brain. Brain Res., 428, 45-52.

López-Sánchez, N., Müller, U. \& Frade, J.M. (2005) Lengthening of $\mathrm{G} 2 /$ mitosis in cortical precursors from mice lacking beta-amyloid precurso protein. Neurascience, 130, 51-60.

Malatesta, P., Hartfuss, E. \& Gotz, M. (2000) Isolation of radial glial cells by fluorescent-activated cell sorting reveals a neuronal lineage. Development, 127, 5253-5263.

Malatesta, P., Hack, M.A., Hartfuss, E., Kettenmann, H., Klinkert, W. Kirchhoff, F. \& Gotz, M. (2003) Neuronal or glial progeny: regional differences in radial glia fate. Neuron, 37, 751-764.

Mandyam, C.D., Norris, R.D. \& Eisch, A.J. (2004) Chronic morphine induces premature mitosis of proliferating cells in the adult mouse subgranular zone

Messier, P.E. \& Auclair, C. (1974) Effect of cytochalasin B on interkinetic nuclear migration in the chick embryo. Dev. Biol., 36, 218-223.

Miller, C.R.D., O'Steen, W.K. \& Deadwyler, S.A. (1982) Effect of morphin on ${ }^{3} \mathrm{H}$-thymidine incorporation in the subependyma of the rat: an autor(log.

Miyama, S., Takahashi, T., Nowakowski, R.S. \& Caviness, V.S. Jr (1997) A gradient in the duration of the Gl phase in the murine neocortica

Miyata, T., Kawaguchi, A., Okano, H. \& Ogawa, M. (2001) Asymmetric Cheritance of radial Alial Ores of cortical Ogeurons. Neuron, 31, 727-741.

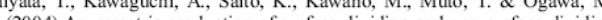
(2) A

Murciano, A. Zamora, J., Lopez-Sanchez, J. \& Fmde J.M. (2002) Interkinetic nuclear movement may provide spatial clues to the regulation of neurogenesis. Mol. Cell. Neurosci., 21, 285-300.

Noctor, S.C., Flint, A.C., Weissman, T.A., Dammerman, R.S. \& Kriegstein $A$. (2001) Neurons derived from radial glial cells establish redial wits in neocortex, Nature, $409,714-720$. Panagiotou, S., Bakogeorgou, E., Papakonstanti, E., Hatzoglou, A., Wallet, F.,
Dussert, C., Stournaras, C. Martin, P.M \& Castanas, E. (1999) Opioid Dason, C., Ston agonists modify breast cancer cell proliferation by blocking cells to the Biochem., 73, 204-211.

Reznikov, K., Hauser, K.F., Nazarevskaja, G., Trunova, Y., Derjabin, V. \& Bakalkin, G. (1999) Opioids modulate cell division in the germinal zone of the late embryonic neocortex. Eur. J. Neurosci, 11, 2711-2719.

Sargeant, T.J., Day, D.J., Mrkusich, E.M., Foo, D.F. \& Miller, J.H. (2007) Mu opioid receptors are expressed on radial glia but not migrating neuroblasts in the late embryonic mouse brain. Brain Res., 1175, 28-39.

C) The Authors (2008). Journal Compilation (c) Federation of European Neuroscience Societies and Blackwell Publishing Ltd

European Journal of Neuroscience, 1 s 
8 T. J. Sargeant et al.

Sauer, F.C. (1935) Mitosis in the neural tube. J. Comp. Neurol., 62, 377-405. Schuller, A.G., King, M.A., Bolan, E., Pan, Y.X., Morgan, D.J., Chang, A. chuller, A.G., King, M.A., Bolan, E., Pan, Y.X., Morgan, D.J., Chang, A., Czick, M.E., Un f mice locking and morphine-6 betarglucuronide analgesia in a

seatriz, J.V. \& wull, 30, 523-527.

Sheng, W.S., Hu, S., Herr, G., Ni, H.T., Rock, R.B., Gekker, G., Lokensgard, J.R. \& Peterson, P.K. (2007) Human neural precursor cells express

kappa opioid receptors. J. Pharmacol. Exp. Ther, 322, 957-963. the early development of the mouse neocortex: a pilot study based on recording the number, location and plane of cleavage of mitotic figures. J. Anat., 116, 67-91.

Spiegel, K., Kourides, I. \& Pasternak, G.W. (1982) Prolactin and growth homone release by momhine in the rat: different receptor mechanisms. Science, 217, 745-747.

Stiene-Martin, A., Zhou, R. \& Hauser, K.F. (1998) Regional, developmental and cell cycle-dependent differences in $\mu, \delta$, and $\kappa$-opioid receptor expression among cultured mouse astrocytes. Glia, 22, 249-259.

Stiene-Martin, A., Knapp, P.E., Martin, K., Gurwell, J.A., Ryan, S., Thornton, S.R., Smith, F.L. \& Hauser, K.F. (2001) Opioid system diversity developing neurons, astroglia and oligodendroglia in the subventricular zone and striatum: impact on gliogenesis in vivo. Glia, 36, 78-88.

Takahashi, T., Nowakowski, R.S. \& Caviness, V.S. Jr (1995) Early ontogeny of the secondary proliferative population of the embryonic murine cerebr wall. J. Neurosci., 15, 6058-6068.
Takahashi, T., Nowakowski, R.S. \& Caviness, V.S. Jr (1996) The leaving or Q fraction of the murine cerebral proliferative epithelium: a general model of neocortical neurogenesis. J. Neurosci., 16, 6183-6196. Takahashi, T., Bhide, P.G., Miyama, S. \& Caviness, V.S. Jr (1999) Proliferative behaviour of the murine cerebral wall in tissue culture: cell cycle kinetics and checkpoints, Exp. Neurol, 156, 407-417.

Tamai, H, Shinohara, H. Takaki, M Saito, K., Nishizawa, Y Nomura, T \& Osumi, N. (2007) Pax 6 transcription factor is required for the interkinetic nuclear movement of neuroepithelial cells. Genes Cells, 12, 983-996. Tripathi, A. Khurshid, N. Kumar, P. \& Iyengar, S. (2008) Expression of delta- and mu-opioid receptors in the ventricular and subventricular of the developing human neocortex. Neurosci. Res., Epub ahead of print ???, ???

Ueno, M., Katayama, K., Yamauchi, H., Nakayama, H. \& Doi, K. (2006) Cell cycle progression is required for nuclear migration of neural progenitor cells. Brain Res., 1088, 57-67.

Vaughan, C.W., Bagley, E.E., Drew, G.M., Schuller, A., Pintar, J.E., Hack, S.P. \& Christie, M.J. (2003) Cellular actions of opioids on periaqueductal grey neurons from $\mathrm{C} 57 \mathrm{Bl} 6 / \mathrm{J}$ mice and mice lacking MOR-1. Br. J. Pharmacol, $139,362-367$.

Xu, B., Kim, S.-T., Lim, D.-S. \& Kastan, M.B. (2002) Two molecularly distinct $\mathrm{G}_{2} / \mathrm{M}$ checkpoints are induced by ionizing irradiation. Mol. Cell. Biol., 22 , 1049-1059.

Zagon, 1.S. \& McLaughlin, PJ. (1983) Increased brain size and cel in infant rats treated with an opiate antagonist. Science, 221, 1179-1180. Zorilla, E.P. (1997) Multiparous species present problems (and possibilities) to developmentalists. Dev. Psychobiol., 30, 141-150. 